\title{
Electrochemical Arsenic Remediation for Rural Bangladesh
}

by

\author{
Susan Elizabeth Amrose Addy
}

B.S. (University of Michigan at Ann Arbor) 2000
M.A. (University of California at Berkeley) 2003

A dissertation submitted in partial satisfaction of the requirements for the degree of

Doctor of Philosophy

in

Physics

in the

GRADUATE DIVISION

of the

UNIVERSITY OF CALIFORNIA, BERKELEY

Committee in charge:

Professor Ashok Gadgil, Co-Chair

Professor Robert Jacobsen, Co-Chair

Professor Richard Muller

Professor David Sedlak

Fall 2008 
The dissertation of Susan Elizabeth Amrose Addy is approved:

Co-Chair Date

Co-Chair Date

Date

Date

University of California, Berkeley 
Electrochemical Arsenic Remediation for Rural Bangladesh

Copyright 2008

by

Susan Elizabeth Amrose Addy 


\begin{abstract}
Electrochemical Arsenic Remediation for Rural Bangladesh

by

Susan Elizabeth Amrose Addy

Doctor of Philosophy in Physics

University of California, Berkeley

Professor Ashok Gadgil, Co-Chair

Professor Robert Jacobsen, Co-Chair
\end{abstract}

Arsenic in drinking water is a major public health problem threatening the lives of over 140 million people worldwide. In Bangladesh alone, up to 57 million people drink arsenic-laden water from shallow wells. ElectroChemical Arsenic Remediation (ECAR) overcomes many of the obstacles that plague current technologies and can be used affordably and on a small-scale, allowing for rapid dissemination into Bangladesh to address this arsenic crisis.

In this work, ECAR was shown to effectively reduce $550-580 \mu \mathrm{g} / \mathrm{L}$ arsenic (including both $\mathrm{As}[\mathrm{III}]$ and $\mathrm{As}[\mathrm{V}]$ in a 1:1 ratio) to below the $\mathrm{WHO}$ recommended maximum limit of $10 \mu \mathrm{g} / \mathrm{L}$ in synthetic Bangladesh groundwater containing relevant concentrations of competitive ions such as phosphate, silicate, and bicarbonate. Arsenic removal capacity was found to be approximately constant within certain ranges of current density, but was found to change substantially between ranges. In order of decreasing arsenic 
removal capacity, the pattern was: $0.02 \mathrm{~mA} / \mathrm{cm}^{2}>0.07 \mathrm{~mA} / \mathrm{cm}^{2}>0.30-1.1 \mathrm{~mA} / \mathrm{cm}^{2}$ $>5.0-100 \mathrm{~mA} / \mathrm{cm}^{2}$. Current processing time was found to effect arsenic removal capacity independent of either charge density or current density. Electrode polarization studies showed no passivation of the electrode in the tested range (up to current density $10 \mathrm{~mA} / \mathrm{cm}^{2}$ ) and ruled out oxygen evolution as the cause of decreasing removal capacity with current density. Simple settling and decantation required approximately 3 days to achieve arsenic removal comparable to filtration with a $0.1 \mu \mathrm{m}$ membrane.

X-ray Absorption Spectroscopy (XAS) showed that (1) there is no significant difference in the arsenic removal mechanism of ECAR during operation at different current densities and (2) the arsenic removal mechanism in ECAR is consistent with arsenate adsorption onto a homogenous Fe(III)oxyhydroxide similar in structure to 2-line ferrihydrite.

ECAR effectively reduced high arsenic concentrations $(100-500 \mu \mathrm{g} / \mathrm{L})$ in real Bangladesh tube well water collected from three regions to below the WHO limit of 10 $\mu \mathrm{g} / \mathrm{L}$. Prototype fabrication and field testing are currently underway.

Professor Ashok Gadgil Dissertation Committee Co-Chair

Professor Robert Jacobsen Dissertation Committee Co-Chair 
This work is dedicated

\author{
to my husband, \\ Nathan Joseph Addy
}

my love, my heart, and my partner in all things,

to my father,

Fredrick James Amrose

who has encouraged me and supported me for my entire life,

and to the friend I will always admire most,

Maya Sophia James

who has saved my life and my sanity and helped shape my outlook on all things. 


\section{Contents}

List of Figures $\quad$ vi

List of Tables $\quad$ xii

1 Introduction 1

1.1 Arsenic contamination . . . . . . . . . . . . . . . . . 1

1.1 .1 Worldwide ....................... 1

1.1.2 Crisis in Bangladesh and West Bengal . . . . . . . . . . . . . 4

1.2 Arsenic remediation and safe water sources . . . . . . . . . . . . 6

1.3 A new implementation model for Bangladesh . . . . . . . . . . . . . 7

1.4 ElectroChemical Arsenic Remediation (ECAR) . . . . . . . . . . . . . 10

1.4.1 ECAR operating parameters . . . . . . . . . . . . . 12

1.4.2 Other considerations - treatment time and cost . . . . . . . . . 13

1.4.3 Previous electrocoagulation research . . . . . . . . . . . . . . . 14

1.4.4 The need for parameter studies in Bangladesh groundwater . . . . 16

1.5 Thesis objectives and dissertation structure . . . . . . . . . . . . . 17

1.5.1 Chapter 2: Relevant Scientific Background . . . . . . . . . . . . . 18

1.5.2 Chapter 3: Chemical and Physical Analysis of Arsenic Complexation with Iron in ECAR . . . . . . . . . . . . . . . . . . 18

1.5.3 Chapter 4: Characterization of Reaction Products . . . . . . . . 20

1.5.4 Chapter 5: ECAR Performance in Real Bangladesh Groundwater . 21

2 Relevant Scientific Background $\quad 23$

2.1 Introduction . . . . . . . . . . . . . . . . . . . . 23

2.2 Arsenic in Natural Waters . . . . . . . . . . . . . . . . 23

2.3 Iron (Hydr)oxides . . . . . . . . . . . . . . . . . . . . 25

2.4 Arsenic sorption onto iron (hydr)oxides . . . . . . . . . . . 30



2.4.2 Review of arsenic adsorption literature . . . . . . . . . . 35

2.4.3 Arsenic removal via zero valent iron . . . . . . . . . . . . . 38

2.4.4 Effect of $\mathrm{pH}$ and speciation on arsenic sorption . . . . . . . . 40 
2.4.5 Kinetics of arsenic sorption . . . . . . . . . . . . . . 43

2.4.6 Effects of co-occuring solutes . . . . . . . . . . . . 48

2.5 Electrocoagulation . . . . . . . . . . . . . . . . . 54

2.5.1 Electrochemical cell theory . . . . . . . . . . . . 54

2.5.2 Electrocoagulation (EC) using iron . . . . . . . . . . . . . . . 62

2.5 .3 As[III] oxidation in EC . . . . . . . . . . . . . . 73

2.5.4 Arsenic removal mechanism using EC . . . . . . . . . . . . . . 75

2.5.5 Arsenic removal studies using EC . . . . . . . . . . . . 75

2.5.6 Kinetics of EC . . . . . . . . . . . . . . . 78

2.5.7 Characterization of EC products . . . . . . . . . . . . 79

2.6 Chapter Summary . . . . . . . . . . . . . . . . . . . . 80

3 Chemical and Physical Analysis of Arsenic Complexation with Iron in $\begin{array}{lr}\text { ECAR } & \mathbf{8 4}\end{array}$

3.1 Introduction . . . . . . . . . . . . . . . . 84

3.1 .1 Operating parameters in ECAR . . . . . . . . . . . 84

3.1 .2 Research Objectives . . . . . . . . . . . . . . . 88

3.2 Methods . . . . . . . . . . . . . . . . . . . . . . 90

3.2.1 Arsenic analysis and arsenic speciation . . . . . . . . . . . . . 90

3.2.2 pH, dissolved oxygen measurements . . . . . . . . . . . . . . . 92

3.2.3 Development of Synthetic Bangladesh Groundwater (SBGW) . . . 93

3.2.4 Electrochemical cell . . . . . . . . . . . . . . . . . . 101

3.2.5 Electrochemical Arsenic Remediation Procedure . . . . . . . . . . 105

3.2.6 ECAR batch experiments run 1 - Matrix 1 . . . . . . . . . . 108

3.2.7 ECAR batch experiments run 2 - Matrix 2 . . . . . . . . . . . 117

3.2.8 Adsorption using post-synthesis ECAR-generated adsorbent . . . . 122

3.2.9 Polarization studies . . . . . . . . . . . . . . . . . . 124

3.2.10 Electrochemical Impedance Spectroscopy . . . . . . . . . . . . . . 128

3.2.11 Sedimentation tests . . . . . . . . . . . . . . . 131

3.3 Results and Discussion . . . . . . . . . . . . . . . . . 133

3.3.1 Resistivity of synthetic Bangladesh water . . . . . . . . . . . 133

3.3.2 Arsenic removal capability in synthetic Bangladesh water . . . . . 136

3.3.3 Effect of charge density . . . . . . . . . . . . . . . . . 143

3.3.4 Effect of current density . . . . . . . . . . . . . . . 146

3.3.5 Effect of current processing time . . . . . . . . . . . . . . 152

3.3.6 Effect of phosphate and silicate on arsenic removal . . . . . . . 154

3.3.7 Parameter trends, tradeoffs, and implications for Bangladesh . . . 157

3.3.8 Adsorption using post-synthesis ECAR-generated adsorbent . . . . 158

3.3.9 As[III] removal in ECAR . . . . . . . . . . . . . . . . . . . 164

3.3 .10 Polarization studies . . . . . . . . . . . . . . . . . 169

3.3.11 Sedimentation versus $0.1 \mu \mathrm{m}$ vacuum filtration . . . . . . . . . . 175

3.4 Chapter Summary . . . . . . . . . . . . . . . . . . . . 180 
4 Characterization of Reaction Products $\quad 189$

4.1 Introduction . . . . . . . . . . . . . . . . . . . . . . . . 189

4.1.1 Research objectives . . . . . . . . . . . . . . . . . . 192

4.2 Methods . . . . . . . . . . . . . . . . . . . . . 194

4.2.1 Arsenic breakthrough as a function of membrane pore size . . . . . 194

4.2.2 Scanning Electron Microscopy (SEM) . . . . . . . . . . . . . . . 194

4.2.3 Chemical analysis of EGA sludge . . . . . . . . . . . . . 195

4.2.4 X-ray Absorption Spectroscopy (XAS) . . . . . . . . . . . . . 196

4.2.4.1 Overview . . . . . . . . . . . . . . 196

4.2.4.2 Iron XANES and XRF-maps . . . . . . . . . . . . 198

4.2.4.3 Null arsenic EXAFS attempts . . . . . . . . . . . . . 202

4.2.4.4 Arsenic k-edge EXAFS . . . . . . . . . . . . . 204

4.2.4.5 Iron k-edge EXAFS . . . . . . . . . . . . . . 206

4.2.4.6 Reference spectra. . . . . . . . . . . . . 207

4.2.4.7 XAS data analysis . . . . . . . . . . . . . . . . 208

4.3 Results and Discussion . . . . . . . . . . . . . . . . . . 209

4.3.1 Limit on the average EGA cluster size . . . . . . . . . . . . 209

4.3.2 Scanning Electron Microscopy (SEM) . . . . . . . . . . . . . . 210

4.3.3 XRF Fe-As mapping of EGA sludge . . . . . . . . . . . . . . . . 214

4.3.4 Chemical analysis of waste EGA sludge . . . . . . . . . . . 215

4.3.5 Iron oxidation state in EGA . . . . . . . . . . . . . . . 218

4.3.6 EGA iron structure - comparison to known iron (hydr)oxides . . . 219

4.3.7 Arsenic bonding structure - comparison to known arsenic-iron com-

plexes . . . . . . . . . . . . . . . . . . 222

4.3.8 EGA iron structure - comparison between current densities . . . . 224

4.3.9 Arsenic bonding structure - comparison between current densities . 226

4.3.10 Arsenic capture in the bulk solution compared to the electrode surface . . . . . . . . . . . . . . . . . . 227

4.4 Chapter Summary . . . . . . . . . . . . . . . . . . . 228

5 ECAR Performance in Real Bangladesh Groundwater 231

5.1 Introduction . . . . . . . . . . . . . . . . . . . 231

5.1 .1 Issues of groundwater transport and storage . . . . . . . . . . . 232

5.1 .2 Research objectives . . . . . . . . . . . . . . . . . 234

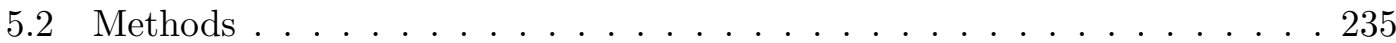

5.2.1 Arsenic analysis and arsenic speciation . . . . . . . . . . . 235

5.2.2 Tube well water sample collection and storage . . . . . . . . . . . 235

5.2.3 Measurements of $\mathrm{As}[\mathrm{III}] / \mathrm{As}_{\text {tot }}$ ratio over time . . . . . . . . . . . . 239

5.2.4 Chemical analysis for co-occuring solutes . . . . . . . . . . . . . 241

5.2 .5 ECAR Treatment . . . . . . . . . . . . . . . . . . . . 242

5.3 Results and Discussion . . . . . . . . . . . . . . . . . 245

5.3.1 As[III $]$ monitoring in samples of real groundwater . . . . . . . . . . 245

5.3.2 ECAR performance in real groundwater . . . . . . . . . . . . 249

5.3.3 Mixing time in real groundwater compared to synthetic groundwater 252 
5.3.4 Sample well comparison to BGS - co-occurring solutes . . . . . . . 256

5.4 Chapter Summary . . . . . . . . . . . . . . . . . . . . . . 262

6 Conclusions $\quad 265$

6.1 Summary of results . . . . . . . . . . . . . . . . . 265

6.2 Future Work . . . . . . . . . . . . . . . . . . . 274

A List of Acronyms 


\section{List of Figures}

1.1 A schematic detailing the roles, cash flow, and mutual benefit relationships between each of the partnering institutions in a public-private partnership (PPP). The PPP is the key to long term viability of a community clean water center. Appropriate safe water technology would be licensed to the private company. . . . . . . . . . . . . . . . . . . .

2.1 Schematic representation of the removal of arsenate ions from solution via EC. For this schematic, $\mathrm{Fe}^{3+}$ ions are dissolving from the electrode, though $\mathrm{Fe}^{2+}$ ions may dissolve as well. In addition, $\mathrm{Fe}(\mathrm{OH})_{3}$ is used to represent the precipitating iron (hydr)oxides, though the actual composition of iron (hydr)oxides formed may be quite complex (see text). . . . . . 68

2.2 Pourbaix diagram for iron. Assumes iron is the only species present. Eh is the equilibrium potential relative to a standard hydrogen electrode (adapted from Hem (1961)) . . . . . . . . . . . . . . .

3.1 The ratio of As[III] to total-arsenic $\left(\mathrm{As}_{t o t}\right)$ over a period of 2-3 days for three representative water batches (WB) of the SBGW-1 type. For water batches 38 and 45, no initial (0 day) measurement of As[III] was done, so average $\mathrm{As}[\mathrm{III}] / \mathrm{As}_{\text {tot }}$ ratio of monitored water batches (8 total) at day-0 was used with the standard deviation as an error. . . . . . . . . . . . 101

3.2 Schematic representation of $850 \mathrm{~mL}$-separated cathode electrochemical cell.103

3.3 Schematic representation of 3 L-single chamber electrochemical cell. . . . 104

3.4 Fraction of remaining arsenic as a function of mixing time for SBGW1. Both kinetics tests were performed under identical conditions after a dosing at $j=1.1 \mathrm{~mA} / \mathrm{cm}^{2}, q=39 \mathrm{C} / \mathrm{L}, t_{C P}=5 \mathrm{~min}$, with initial arsenic

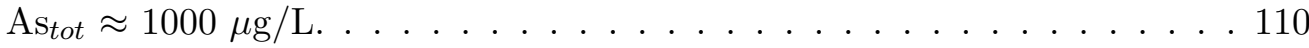


3.5 Replicate variability between arsenic concentrations measured in identical batch tests (repeat measurements) for Matrix 1 (where repeats are available) as a function of the average final arsenic concentration, $A s_{\text {fave }}$. The starred point has a higher than expected standard deviation, and represents the batch test performed at $j=30 \mathrm{~mA} / \mathrm{cm}^{2}$ and $t_{C P}=5 \mathrm{~min}$. The dotted line in a linear fit to non-starred points. . . . . . . . . . . . . . 114

3.6 Mixing time kinetics profile for SBGW-2 spiked to $\mathrm{As}_{t o t}=510 \mu \mathrm{g} / \mathrm{L}$ treated at $j=1.1 \mathrm{~mA} / \mathrm{cm}^{2}, q=150 \mathrm{C} / \mathrm{L}, t_{C P}=66 \mathrm{~min}$. Residual arsenic concentration (left axis) and percent arsenic removed (right axis) as a function of mixing time are shown. A dashed horizontal line marks the WHO limit $(10 \mu \mathrm{g} / \mathrm{L}) \ldots \ldots \ldots \ldots$

3.7 Deviation in the final arsenic concentration measured between replicated batch tests for Matrix 2 as a function of the average final arsenic concentration, $A s_{\text {fave }}$. The deviation plotted between two measurement $y_{1}$ and $y_{2}$ is just $\sigma=\left|y_{2}-y_{1}\right| / \sqrt{2}$. The dashed line in a linear fit weighted by

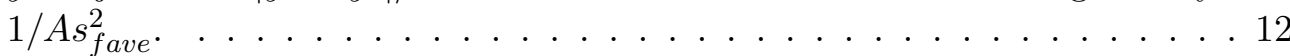

3.8 Schematic of Three-Electrode Cell used for polarization studies and Electronic Impedance Spectroscopy (EIS). The cell contained a platinum counter electrode, an $\mathrm{Ag} / \mathrm{AgCl}$ reference electrode, and an exposed iron disk working electrode (surface area $0.025 \mathrm{~cm}^{2}$ ). The cell is described more fully in the text (Section 3.2.9) . . . . . . . . . . . . . . . . . 126

3.9 A simple Nyquist plot showing complex impedance vector $Z(\omega)$. Note that the $\mathrm{Y}$-axis is negative and that each point on the Nyquist plot is the impedance at one frequency, $\omega$. In this plot, low frequencies appear on the right side and high frequencies appear on the left. This is the case when the impedance falls as the frequency rises (usually, but not always, true). . . . . . . . . . . . . . . . . . . . 129

3.10 A Nyquist plot for a representative electrochemical impedance scan about the open circuit potential in synthetic Bangladesh groundwater (SBGW-1 recipe).

3.11 Equivalent circuit used in EIS analysis to measure electrolyte resistance of synthetic Bangladesh groundwater. Components include the double-layer capacitance, $C_{d l}$, the charge-transfer resistance, $R_{c t}$, and the electrolyte resistance, $R_{e} \ldots \ldots \ldots \ldots \ldots \ldots$. . . . . . . . . . . . . . . . . . .

3.12 Arsenic removal (concentration on left vertical axis; percent arsenic removed on right vertical axis) as a function of charge density, $q$, after ECAR treatment at current densities $0.07-1.1 \mathrm{~mA} / \mathrm{cm}^{2}$ of low phosphate, zero silicate synthetic Bangladesh groundwater (SBGW-1 recipe -

3.13 Arsenic removal (concentration on left vertical axis; percent arsenic removed on right vertical axis) as a function of charge density, $q$, after ECAR treatment at current densities $0.02-100 \mathrm{~mA} / \mathrm{cm}^{2}$ of average phosphate, average silicate synthetic Bangladesh groundwater (SBGW-2 recipe - Matrix 2 conditions). . . . . . . . . . . . . . . . . . . . . . . 140 
3.14 Arsenic removal (concentration on left vertical axis; percent arsenic removed on right vertical axis) as a function current processing time for Matrix 1 batch experiments for current densities $j=0.07,0.30,0.70$, and $1.1 \mathrm{~mA} / \mathrm{cm}^{2}$. Also included are batch tests performed with no external voltage (blanks). . . . . . . . . . . . . . . . . . . . . . . 149

3.15 Arsenic removal (concentration on left vertical axis; percent arsenic removed on right vertical axis) as a function of charge density, $q$, for three batch tests; $j=0.02 \mathrm{~mA} / \mathrm{cm}^{2}$ with $t_{C \text { Ptotal }}=19.4$ hours, $j=1.1 \mathrm{~mA} / \mathrm{cm}^{2}$ with the same $t_{C P t o t a l}=19.4$ hours, and the original Matrix 2 batch test run for $j=1.1 \mathrm{~mA} / \mathrm{cm}^{2}$ using the much shorter $t_{C \text { Ptotal }}=1.2$ hours. . . 151

3.16 Arsenic removal (normalized concentration on left vertical axis; percent arsenic removed on right vertical axis) as a function of charge density, $q$, for two batch tests at $j=0.02 \mathrm{~mA} / \mathrm{cm}^{2}$ in which the only difference is a decrease in $t_{C P}$ by $60 \%$ for the Fast test relative to the slow tests. Both batch tests used Matrix 2 procedures and SBGW-2 water with initial

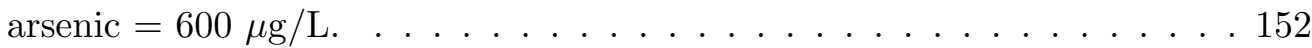

3.17 Arsenic removal (concentration on left vertical axis; percent arsenic removed on right vertical axis) as a function of charge density, $q$, for low phosphate, zero silicate synthetic Bangladesh groundwater (SBGW-1, using Matrix 1 procedure) and average phosphate, average silicate synthetic Bangladesh groundwater (SBGW-2, using Matrix 2 procedure). ECAR treatment occured at $j=1.1 \mathrm{~mA} / \mathrm{cm}^{2}$ in both cases. . . . . . . . . . . 155

3.18 Arsenic removal (concentration on left vertical axis; percent arsenic removed on right vertical axis) as a function of contact time for PSA experiments using post-synthesis ECAR-generated adsorbents, both freshly prepared (PSA-Fresh) and aged for 60 minutes (PSA-Aged $60 \mathrm{~min}$ ). Adsorbent was generated using ECAR operating conditions $j=5.0 \mathrm{~mA} / \mathrm{cm}^{2}$, $q=175 \mathrm{C} / \mathrm{L}, t_{C P}=17.27 \mathrm{~min}$ in arsenic-free SBGW-2 water. . . . . . . 160

3.19 Arsenic removal (concentration on left vertical axis; percent arsenic removed on right vertical axis) as a function of quiescent contact time for PSA experiments using post-synthesis ECAR-generated adsorbents, both freshly prepared (PSA-Fresh) and aged for 60 minutes (PSA-Aged $60 \mathrm{~min}$ ), and compared to ECAR treatment at the same current density $\left(j=5.0 \mathrm{~mA} / \mathrm{cm}^{2}\right)$ and a similar current processing time $\left(t_{C P}=16.33 \mathrm{~min}\right)$ as that used to generate the PSA adsorbents. This is a continuation of Figure 3.18 (note however the change in x-axis units). In all cases, quiescent contact began after a period of active mixing $\left(t_{M}=60 \mathrm{~min}\right.$ in the case of ECAR treatment, and $t_{M}=120$ minutes in the case of PSA experiments). Initial arsenic concentration in all three cases was $\mathrm{As}_{t o t}=$ $590 \pm 40 \mu \mathrm{g} / \mathrm{L} \ldots \ldots \ldots \ldots 1 . \ldots \ldots \ldots$ 
3.20 Post-treatment As[III] and As[V] (concentration on left vertical axis; percent arsenic removed on right vertical axis) as a function of charge density, $q$. This batch test was run on SBGW-2 water with operating conditions $j=1.1 \mathrm{~mA} / \mathrm{cm}^{2}, t_{C P}=66.58 \mathrm{~min}$, Matrix 2 procedure. These results were typical of all current densities in Matrix 2 (i.e. 0.02, 1.1, 5.0, 10, 30, and $\left.100 \mathrm{~mA} / \mathrm{cm}^{2}\right) \ldots \ldots \ldots \ldots \ldots$. . . . . . . . . . . . . . . . . . . . . . .

3.21 Comparison of the final As[III] concentration (initial concentration 300 $\mu \mathrm{g} / \mathrm{L})$ for SBGW-2 water after ECAR treatment to adsorption with freshly made post-synthesis ECAR-generated adsorbent (PSA-Fresh) and aged post-synthesis ECAR-generated adsorbent (PSA-Aged). ECAR treatment was performed under similar conditions to the generation of postsynthesis adsorbent. See Section 3.3.8 and Section 3.2.8 for experimental conditions. . . . . . . . . . . . . . . . . 166

3.22 Anodic polarization curve for iron wire electrode in $0.1 \mathrm{M}^{\mathrm{KClO}_{4}}$ (thin line) and $0.1 \mathrm{M} \mathrm{KClO}_{4}+\mathrm{SBGW}$ (thick line) scanned at $\nu=0.1 \mathrm{mV} / \mathrm{s}$. Horizontal lines indicate the current densities used in Matrix 1 (dotted) and Matrix 2 (dashed) experiments. Thermodynamic equilibrium potentials at $25^{\circ} \mathrm{C}$ for appropriate redox couples are shown for $\mathrm{pH}=7$ (in red) expected of natural groundwater - and $\mathrm{pH}=9$ (in blue) - close to the $\mathrm{pH}$ of the actual scan. A pourbaix diagram composed by Bang et al. (2005a) assuming solid $\mathrm{Fe}, \mathrm{Fe}(\mathrm{OH})_{2}$ and $\mathrm{Fe}(\mathrm{OH})_{3}$ exist was consulted to select appropriate lines at each $\mathrm{pH}$. If concentration appears in the equilibrium calculation, a range of concentrations from $10^{-8} \mathrm{M}$ to $1 \mathrm{M}$ are shown. . . . 170

3.23 Comparison of the final arsenic concentration for $0.1 \mu \mathrm{m}$ filtered and sedimentation samples from the Matrix 1 series of experiments. Ts, listed below each series of bars, is the quiescent settling time allowed before decantation for the settled sample $(0.1 \mu \mathrm{m}$ filtered samples were all filtered immediately after mixing). In the case of series 4 (right), a second sample was removed and tested from the sedimentation beaker at a later time. The ECAR operating conditions for the above experiments were (1) $j=$ $1.1 \mathrm{~mA} / \mathrm{cm}^{2}, q=23 \mathrm{C} / \mathrm{L}, t_{C P}=3 \mathrm{~min} ;(2) j=1.1 \mathrm{~mA} / \mathrm{cm}^{2}, q=85$ $\mathrm{C} / \mathrm{L}, t_{C P}=5 \mathrm{~min} ;(3) j=0.07 \mathrm{~mA} / \mathrm{cm}^{2}, q=25 \mathrm{C} / \mathrm{L}, t_{C P}=50 \mathrm{~min}$; and (4) $j=0.70 \mathrm{~mA} / \mathrm{cm}^{2}, q=15 \mathrm{C} / \mathrm{L}, t_{C P}=3 \mathrm{~min}$. Each series has been normalized to its average initial arsenic concentration to avoid small deviations due to different starting arsenic levels - the average initial arsenic concentration was $610 \pm 40 \mu \mathrm{g} / \mathrm{L} . \ldots \ldots 176$

3.24 The difference in arsenic concentration between samples filtered through a $0.1 \mu \mathrm{m}$ membrane and samples decanted from the top of a beaker after ECAR treatment $\left(j=1.1 . \mathrm{mA} / \mathrm{cm}^{2}, q=100 \mathrm{C} / \mathrm{L}\right)$ following a period of quiescent settling as a function of the quiescent settling time. Two tests are shown in which the solution was mixed for 30 minutes before quiescent settling (solid line, solid symbols) and mixed for 60 minutes before quiescent settling (dashed line, open symbols) . . . . . . . . . . . 177 
4.1 Arsenic concentration measured in filtrate (normalized to the highest average value) after ECAR-treated sample water passed through membranes of different pore size. Errors represent the variability between duplicate tests. . . . . . . . . . . . . . . . . . . . . . . 209

4.2 Scanning electron micrographs of ECAR generated sludge. Image $A$ is of air-dried sludge collected on a membrane and magnified 500x. The two marked areas in image $A$ are imaged at 8,000 x (image $B$ ) highlighting a representative square crystal and at 50,000x and 100,000x (image $C$ and $D$ respectively), highlighting a representative area of dry caked powder. . 212

4.3 Energy dispersive spectra taken during SEM imaging of (A) the bare polyvinylidene fluoride membrane (background), (B) a further magnified image of Figure 4.2B (square crystal) centered on a bare section of crystal, and $(\mathrm{C})$ the area shown in Figure 4.2D (caked powder). Au lines appear in all spectra due to the gold coating sputtered onto the sample to facilitate SEM imaging. . . . . . . . . . . . . . . . . . . . . . . . 213

4.4 XRF bicolor map of EGA powder. In this picture, each pixel is colored red in proportion to the As signal and green in proportion to the Fe signal (pixels with both arsenic and iron appear yellow). This map was measured for EGA generated at $j=1.1 \mathrm{~mA} / \mathrm{cm}^{2}$. Maps of EGA generated at $j=$ 0.02 and $5.0 \mathrm{~mA} / \mathrm{cm}^{2}$ were similar. . . . . . . . . . . . . . 215

4.5 Normalized Fe XANES spectra for EGA sludge after ECAR treatment at several different current densities $\left(j=0.02,0.07,1.1\right.$, and $\left.5.0 \mathrm{~mA} / \mathrm{cm}^{2}\right)$ are shown along with Fe XANES spectra for various iron (hydr)oxides reference compounds, including Fe[III](hydr)oxides 2-line ferrihydrite, 6-line ferrihydrite, goethite, lepidocrocite, and akaganeite and Fe(II, III)(hydr)oxides (average oxidation number 2.67) green rust (carbonate) and green rust (sulfate) . . . . . . . . . . . . . . . . . . . . 220

4.6 Iron EXAFS spectra (weighted by $\mathrm{k}^{3}$ ) for EGA generated at $j=1.1 \mathrm{~mA} / \mathrm{cm}^{2}$ compared to reference spectra for iron (hydr)oxides 2-line ferrihydrite $(2 \mathrm{~L}$ FH), goethite, and scorodite. Arrows note significant differences between spectra. . . . . . . . . . . . . . . . . . . . . 221

4.7 Arsenic EXAFS spectrum (weighted by $\mathrm{k}^{3}$ ) for arsenic-laden EGA generated at $j=1.1 \mathrm{~mA} / \mathrm{cm}^{2}$ compared to reference spectra for As $[\mathrm{V}]$ adsorbed onto goethtite, $\mathrm{As}[\mathrm{V}]$ adsorbed onto 2-line ferrihydrite $(2 \mathrm{~L} \mathrm{FH})$, and the mineral scorodite $\left(\mathrm{FeAsO}_{4}\right)$. Arrows indicate spectral differences. . . . . . 223

4.8 Iron EXAFS spectra (weighted by $\mathrm{k}^{3}$ ) for EGA generated at current densities $j=100 \mathrm{~mA} / \mathrm{cm}^{2}, 5.0 \mathrm{~mA} / \mathrm{cm}^{2}, 1.1 \mathrm{~mA} / \mathrm{cm}^{2}$, and $0.02 \mathrm{~mA} / \mathrm{cm}^{2}$ (displayed top to bottom). . . . . . . . . . . . . . . . . . 224

4.9 Arsenic EXAFS spectra (weighted by $\mathrm{k}^{3}$ ) for EGA generated at current densities $j=100 \mathrm{~mA} / \mathrm{cm}^{2}, 5.0 \mathrm{~mA} / \mathrm{cm}^{2}, 1.1 \mathrm{~mA} / \mathrm{cm}^{2}$, and $0.02 \mathrm{~mA} / \mathrm{cm}^{2}$ (displayed top to bottom) . . . . . . . . . . . . . 226

5.1 Map of Bangladesh highlighting areas visited. . . . . . . . . . . . . 236 
5.2 Percent $\mathrm{As}[\mathrm{III}]$ relative to initial concentration remaining with time for three synthetic Bangladesh groundwater batches (WB; open symbols, dotted lines) and tube well water sample 12 (TW 12; solid symbol, solid line). The initial As[III] concentration for WB 38 and 45 was not measured, so the value is assumed to be the average As[III] concentration for all measured water batches, As[III] $=304 \pm 41 \mu \mathrm{g} / \mathrm{L}$. This uncertainty appears in all subsequent points, since the measured As[III] concentration must be divided by the initial As[III] concentration to calculate the percentage As[III $]$ remaining. The uncertainty in As[III $] / \mathrm{As}[\mathrm{III}]_{\text {initial }}$ for TW 12 is estimated as the percentage decrease in $\mathrm{As}_{\text {tot }}$ relative to the initial concentration plus the ICPMS measurement uncertainty for both As[III] and As $[\mathrm{III}]_{\text {initial }} \ldots \ldots \ldots \ldots$. . . . . . . . . . . . . . . . . . . . 248

5.3 Arsenic concentrations before and after ECAR treatment for groundwater from four tube wells in Bangladesh along with SBGW-1 synthetic groundwater (SBGW), plus results from filtration alone (no ECAR) of TW 10 after 12 days of settling. Post-treatment concentrations are given for samples filtered immediately after Coulombic dosing (i.e. no mixing, of $t_{M}=$ 0 min) and after more than 15 min mixing $\left(t_{M}>15\right.$ min, see Table 5.3 for mixing times). Note that only Quick Test arsenic measurements are available for TW $4, t_{M}=0 \mathrm{~min}$; all other measurements are via ICPMS. The initial arsenic concentration for $T W 10$ and Filtered only $T W 10$ refers to the arsenic concentration immediately before ECAR treatment and 0.7 hrs after collection from the tube well respectively. . . . . . . . . . . . 251

5.4 Residual arsenic concentration as function of mixing time $\left(t_{M}\right)$ for SBGW1 synthetic groundwater $\left(\mathrm{As}_{\text {initial }}=530 \mu \mathrm{g} / \mathrm{L}\right)$ and real groundwater from Tube Well $4\left(\mathrm{TW} 4 ; \mathrm{As}_{\text {initial }}=93 \mu \mathrm{g} / \mathrm{L}\right)$. Note that points overlap at $t_{M}$ $=30 \mathrm{~min}$. All arsenic concentrations were measured using ICPMS except for TW $4, t_{M}=0 \mathrm{~min}$, which was measured using the Quick Test (hence the higher error). Error in mixing time is $+3 \mathrm{~min}$ for all points due to time of filtration. . . . . . . . . . . . . . . . . . 2 . 254 


\section{List of Tables}

1.1 List of countries that have reported arsenic contamination in the groundwater supply as of 2007 (based on (Petrusevski et al., 2007)) . . . . . . . 2

2.1 The iron (hydr)oxides. Adapted from Cornell \& Schwertmann (2000) . . . 29

3.1 Synthetic groundwater composition and corresponding mean values and standard deviations of groundwater in Bangladesh, obtained from the BGS database (values for $\mathrm{pH}, \mathrm{As}[\mathrm{III}], \mathrm{As}[\mathrm{III}] / \mathrm{As}_{\text {tot }}, \mathrm{HCO}_{3}^{-}$, and $\mathrm{Cl}^{-}$taken from special Study Areas; all other values were taken from National Survey data, using only wells with As $>10 \mu \mathrm{g} / \mathrm{L}$ for data analysis, and setting values below detection limits to zero). For each solute, the percentage of wells in the BGS database with a concentration $(x)$ less than that in the synthetic groundwater 1 and $2\left(x_{s 1}, x_{s 2}\right)$ is given. Values for $\mathrm{pH}, \mathrm{As}$, $\mathrm{As}[\mathrm{III}]$, and $\mathrm{As}[\mathrm{III}] / \mathrm{As}_{\text {tot }}$ are measured values across water batches, all other ions are spiked values. . . . . . . . . . . . . . . . . 95

3.2 Operating parameters for Matrix 2 tests. . . . . . . . . . . . . 118

3.3 Results of ECAR batch tests in synthetic Bangladesh groundwater (SBGW1) containing low phosphate and no silicate. Initial and residual arsenic concentrations are averaged over replicate batch tests. Boldface indicates the lowest residual arsenic concentration achieved for each current density. Errors on arsenic measurements are 10\%. . . . . . . . . . . . . 137

3.4 Results of ECAR batch tests in synthetic Bangladesh groundwater SBGW2 , including the average concentrations of phosphate and silicate found in Bangladesh. Initial and residual arsenic concentrations are averaged over replicate batch tests. Boldface indicates the lowest residual arsenic concentration acheived for each current density. Errors on arsenic measurements are $10 \% \ldots \ldots \ldots 139$ 
3.5 Performance metrics to reach the Bangladesh arsenic limit $(50 \mu \mathrm{g} / \mathrm{L})$ and the WHO arsenic limit $(10 \mu \mathrm{g} / \mathrm{L})$ based on ECAR batch tests in synthetic Bangladesh groundwater containing a 1:1 ratio of As[III]:As[V]. Arsenic removal capacity, iron capacity (assuming all is dissolved as $\mathrm{Fe}^{3+}$ ), and total treatment time have been calculated based on the WHO limit and data in Figures 3.12 and 3.13. . . . . . . . . . . . . . . . . . . . . . 144

3.6 Comparison of arsenic removal capability and arsenic removal capacity using ECAR treatment versus treatment with freshly made (PSA-fresh) and aged 60 min (PSA-aged) post-synthesis ECAR-generated adsorbents. Arsenic removal capacity has been calculated assuming that all of the applied charge (during ECAR treatment or ECAR-generation of adsorbent) is used to form $\mathrm{Fe}^{3+}$ ions in solution. . . . . . . . . . . . . . . 162

4.1 ECAR operating conditions used to generate XAS samples. ECAR operating conditions (summarized in Section 1.4.1) include current density, charge density, current processing time $\left(t_{C P}\right)$, and mixing time $\left(t_{M}\right)$. XAS sample preparation procedures are described in Sections 4.2.4.2 - 4.2.4.5. . 201

4.2 Chemical analysis of waste EGA sludge or electrode scrapings from ECAR treatment at various operating conditions. Sample preparation is described in Section 4.2.3. . . . . . . . . . . . . . . . . . 216

5.1 Tube well locations, initial arsenic concentration and time of initial aliquot, and list of tests performed on each tube well water sample. . . . . . . . . 239

$5.2 \mathrm{As}_{t o t}, \mathrm{As}[\mathrm{III}]$, and $\mathrm{As}[\mathrm{III}] / \mathrm{As}_{\text {tot }}$ ratio for TW 12 up to 7 days after collection. 245

5.3 ECAR operating conditions and results for treatment of tube well samples and SBGW-1 synthetic groundwater. . . . . . . . . . . . . . . 250

5.4 Groundwater composition of selected tube wells (TW) after 11-19 days of storage compared to BGS statistics from the same region. Synthetic groundwater parameters used in batch tests (SBGW-1,2) are added for comparison (from Table 3.1). BGS statistics include all wells available in the designated region with $\mathrm{As}_{\text {tot }} \geq 10 \mu \mathrm{g} / \mathrm{L}$ (standard deviation given in parenthesis). Italics indicate a concentration that falls outside of the range defined by the BGS regional average value \pm the standard deviation. 257 


\section{Acknowledgments}

I am indebted to my husband Nathan for his tolerance of endless edits, complaints, and long days. Thank you Nathan for always believing in me, for your endless support everyday, and for never letting go.

I am grateful for the constant support of my sister, Kathy Amrose Larsen, who helped me get to the finish. I am also grateful for a lifetime of support from my parents, Judy and Fred Amrose, who never gave up on me and allowed me to find my own way. Thanks also to my twin, Steven Amrose.

I would like to thank my research advisor, Professor Ashok Gadgil, for showing me that physics can make a difference and for continuing to teach me how. I would like to thank Professor Bob Jacobsen for never giving up on me and Professor Marjorie Shapiro for her support of my topic. I am grateful for the support of my committee members, Professor David Sedlak and Professor Rich Muller, who gave many thoughtful comments. I would also like to thank Art Rosenfeld, who participated in my qualifying exam and has been an inspiration to me. A special thanks to Professor Steven Boggs.

I am grateful for the excellent mentoring of Dr. Robert Kostecki, who offered many hours of advice and patience, and who kindly offered his research space for my thesis work. I am also grateful for the guidance and advice of Dr. Venkat Srinivasan, Dr. Lara Gundel, Jonathan Slack and Howdy Goudy. Thanks to postdoctoral researchers Dr. 
Laurence Hardwick and Dr. Marek Marcinek for help and patience in the lab. Thanks to Johanna Mathieu for many useful critiques, comments, advice, and support. I have had the honor of working with many talented undergraduates on this work, including Kate Ming, Scott Mclaughlin, Yola Bayram and Emily Desley-Bloom. A special thanks to Kristin Kowolik. Thanks also to the wonderful student BEAR team - Marc Muller, Jessica Huang, Debbie Cheng, Marianna Kowalczyk, John Wang, and Michele Itten.

I would like to thank Matthew Marcus, John Bargar, and Sirine Fakra for support and advice during EXAFS data collection and analysis. Thanks to Dr. Peggy O'Day and Dr. Andrea Foster for sharing advice and EXAFS spectra. A special thanks to Jasquelin Pena and Dr. Brandy Toner, who sat down with me to explain EXAFS procedures and theory and offered wonderful guidance and advice.

My research funding was provided by the LDRD (Laboratory Directed Research and Development) program at Lawrence Berkeley National Lab, the Blum Center for Developing Economies, the EPA P3: People, Prosperity and the Planet Award, and the Haas School of Business Sustainable Projects and Solutions Program. I am grateful for the attention and inspiration provided by George Scharffenberger. I am also grateful for the administrative assistance of Jane Youn, Lee Borrowman, Donna Sakima, Anne Takizawa, Claudia Trujillo - I thank you all wholeheartedly for all of your help.

This work was supported by the U.S. Department of Energy under Contract No. DE-AC02-05CH11231. 


\section{Chapter 1}

\section{Introduction}

\subsection{Arsenic contamination}

\subsubsection{Worldwide}

Arsenic in drinking water is a major public health problem threatening the lives of over 140 million people worldwide (Kahn, 2007; Beck, 2007). The acute toxicity of arsenic in high concentrations has been known about for centuries, but only recently has a strong adverse effect on health been associated with long-term exposure to even very low concentrations (Petrusevski et al., 2007). Over the past few decades, naturallyoccurring arsenic has been discovered in groundwater used to supply drinking water in many countries on all continents. Well-known high arsenic groundwater areas have been found in Argentina, Chile, Mexico, China and Hungary, and more recently in West Bengal (India), Bangladesh and Vietnam (a full list is presented in Table 1.1). Arsenic is colorless, tasteless, and odorless, and accumulates in the human body over time, making 
Table 1.1: List of countries that have reported arsenic contamination in the groundwater supply as of 2007 (based on (Petrusevski et al., 2007)).

Continent Countries Reporting Arsenic in Groundwater

Asia Bangladesh, Cambodia, China (including provinces of Taiwan and Inner Mongolia), India, Iran, Japan, Myanmar, Nepal, Pakistan, Thailand, Vietnam

Americas Argentina, Chile, Dominica, El Salvador, Honduras, Mexico, Nicaragua, Peru, US (including Alaska)

Europe Austria, Croatia, Finland, France, Germany, Greece, Hungary, Italy, Romania, Russia, Serbia, United Kingdom

Africa Ghana, South Africa, Zimbabwe

Pacific Australia, New Zealand

it an insidious threat to public health.

Arsenic poisoning is painful and disfiguring. Skin lesions (known as Keratosis) often appear first, with a gestation period of 5 to 15 years, commonly causing dark pigmentation of the skin and a hardening of the palms and feet (Madajewicz et al., 2008). Associated symptoms can include weakness, lethargy and fatigue that can prevent work, chronic respiratory problems, including painful bloody coughing that can be misdiagnosed as TB, gastrointestinal problems, cardiovascular disease and neuropathy, which includes tremors, headaches, vertigo, numbness, and pain (Chowdhury et al., 2000). Arsenic may also cause developmental problems in children (Wasserman et al., 2004). The hardening of the skin associated with skin lesions can be so severe that the flesh may crack, and gangrene can set in causing the victim to lose his or her limbs (Madajewicz 
et al., 2008).

Long-term effects of arsenic exposure are known to include liver, lung, kidney and bladder malfunctions along with hypertension, strokes, and heart disease (Chang et al., 2004; Chiu et al., 2004; Moore et al., 1997). Recently, exposure to low levels of arsenic has been linked to a prevalence of Type II diabetes in the US (Navas-Acien et al., 2008; Kyle \& Christiani, 2008). Eventually, exposure leads to cancers of the skin, lungs, bladder, urinary tract, and kidney. It has been estimated that the risk of dying from cancer due to chronic exposure to $50 \mu \mathrm{g} / \mathrm{l}$ arsenic is as high as 13 in 1000 (Smith et al., 2000). The effects of arsenic poisoning are cumulative and can only be reversed up to a point. In some cases, even complete elimination of exposure cannot reverse the effects ${ }^{1}$ (Hopenhayn-Rich et al., 1996).

The range of arsenic concentrations found in natural waters is large, ranging from less than $0.5 \mu \mathrm{g} / \mathrm{L}$ to more than $5000 \mu \mathrm{g} / \mathrm{L}($ Smedley \& Kinniburgh, 2002). Typical freshwater concentrations are less than $10 \mu \mathrm{g} / \mathrm{L}$, though occasionally, much higher concentrations are found, particularly in groundwater ${ }^{2}$. Increased knowledge of the cancer risk led the World Health Organization (WHO) to lower its recommended maximum limit of arsenic from $50 \mu \mathrm{g} / \mathrm{L}$ to $10 \mu \mathrm{g} / \mathrm{L}$ in drinking water (WHO, 1993).

\footnotetext{
${ }^{1}$ For more information on the health effects of arsenic exposure, see Saha et al. (1999), Ahsan et al. (2006), or (Petrusevski et al., 2007) and references therein.

${ }^{2}$ Smedley \& Kinniburgh (2002) offers an excellent review of the factors controlling arsenic concentrations in natural waters.
} 


\subsubsection{Crisis in Bangladesh and West Bengal}

The scope and potential ramifications of arsenic contamination are greatest in Bangladesh, where up to 57 million people daily are exposed to arsenic levels greater than $10 \mu \mathrm{g} / \mathrm{L}$, in some cases as high as 2,500 $\mu \mathrm{g} / \mathrm{L}$ BGS (2001); Smith et al. (2000). The bordering region of West Bengal, India is believed to have an additional 6 million people exposed to arsenic levels of 50 - 3,200 $\mu \mathrm{g} / \mathrm{L}$ (BGS, 2001; WHO, 2004). These estimates come from a widespread detailed survey of groundwater conducted in 1998/1999 in Bangladesh by the British Geological Survey (BGS), demonstrating that $46 \%$ of shallow tube wells $(<150 \mathrm{~m})$ have arsenic concentrations exceeding the WHO guideline of $10 \mu \mathrm{g} / \mathrm{L}$. In 2006, UNICEF similarly reported $32 \%$ of wells tested so far exceed $50 \mu \mathrm{g} / \mathrm{L}$ (UNICEF, 2006). The Water and Sanitation Program South Asia Office (WSP-SA) estimated 20-40 million people in Bangladesh were ingesting unsafe levels of arsenic in 2000 (WSP-SA, 2000). The extent of this problem has led the WHO (Smith et al., 2000) to describe the situation as "the largest mass poisoning of a population in history... beyond the accidents at Bhopal, India, in 1984, and Chernobyl, Ukraine, in 1986.”

The arsenic present in the groundwater of Bangladesh is generally believed to have been released during weathering and adsorbed onto naturally occurring neoformed iron oxyhydroxides. Bacteria are thought to have reductively dissolved the iron oxyhydroxide coatings using available organic matter, thereby releasing arsenic into solution (Nickson et al., 2000; BGS, 2001; Harvey et al., 2002; Islam et al., 2004, 2005). Arsenic release due to in-situ oxidation of sulfide materials in response to lowering water

levels (due to extended tube well use) has been proposed, but largely dismissed due to 
the lack of correlation between arsenic and sulfate (Lowers et al., 2007). Contaminated groundwater has only recently been accessed for drinking. Until the early 1970s, most of the Bangladeshi population was drinking from shallow hand-dug wells, rivers, and ponds. These dug wells were not contaminated with arsenic, but were associated with cholera, diarrhea, and dysentery due to microbial contamination of the surface water (Kaufmann et al., 2001). In the 1970s and 1980s, UNICEF and other international agencies responded by helping to install more than 4 million hand-pumped tube wells in Bangladesh, reportedly converting 80\% of the population by 1997 (UNICEF, 1999). In 1983, the first cases of arsenic-induced skin lesions were identified in the West Bengal region (Saha, 1995) and by 1993, arsenic in tube wells was identified as the cause. In 2006, the number of people already showing symptoms of arsenic poisoning was estimated at 40,000 with predictions that it could soon rise to 1 million (UNICEF, 2006).

Arsenic poisoning has an even heavier toll in the midst of poverty. Bad nutrition is known to exacerbate its toxic effects, especially in children (Rahman et al., 2005). In addition, increased health costs and loss of productivity and income due to symptoms of arsenic poisoning can become perilous to an individual and their entire family at subsistence-level living. The welfare costs of exposure to arsenic have been estimated at US $\$ 84$ per household per year in neighboring parts of India (Roy, 2008) and are likely to be similar in Bangladesh. Such a cost can be crippling for a family living on less than US $\$ 1$ per day. Other indirect effects include social exclusion because of visible skin lesions, additional hours of walking to distant potentially safe tube wells (usually by women and girls), and social strain as neighbors must negotiate for scarce safe water 
sources that are usually privately owned (WHO, 2000).

\subsection{Arsenic remediation and safe water sources}

In developed countries, there are several proven technical approaches for removing arsenic from municipal water supplies. These include: (1) oxidation and stripping, (2) coagulation, precipitation, and filtration using iron and aluminum salts, (3) lime softening, (4) ion exchange resins, (5) membrane processes (e.g. reverse osmosis), and (6) adsorption $^{3}$ onto commercially-available activated alumina, activated carbon, activated bauxite, or Granular Ferric Hydroxide (GFH). Each of these has drawbacks for applicability to highly dispersed, very poor populations with low levels of literacy and formal education, including high cost and large amounts of toxic waste. Reviews of these technologies can be found in Bissen \& Frimmel (2003) and Ng et al. (2004).

There is no traditional technology in Bangladesh capable of removing arsenic. Safe water approaches being tried or considered suitable for developing countries fall into two primary categories - switching to alternative arsenic-free water sources and remediation of arsenic contaminated sources. In Bangladesh, the former category includes installation of deep tubewells (which are more likely to be arsenic-free), treatment of surface waters (e.g. Pond Sand Filters or shallow dugwells), and rainwater harvesting. Arsenic remediation efforts have primarily focused on point-of-use filters for contaminated wells. Arsenic removal is often based on adsorption onto a relatively low-cost chemical sorbent added to the water, such as zero-valent iron, granular ferric hydroxide

\footnotetext{
${ }^{3}$ Adsorption is defined and discussed more thoroughly in Chapter 2.
} 
or activated alumina. Each safe water option varies in capital and recurring costs, levels of maintenance, water quality, water flow rate, social acceptability and other external factors.

Of the arsenic-free sources, the installation of deep tubewells is a strong preference (Hoque et al., 2004). However, deep tubewells are expensive, are only feasible where deep aquifers exist, and must be installed properly to avoid arsenic contamination from shallow aquifers. There is also concern that increased pumping from deep aquifers could draw arsenic in from shallow counterparts (Yu, 2003). Point-of-use filters have been plagued by high abandonment rates after a short time (Ahmad et al., 2003; Hoque et al., 2004; Vergara, 2007), due to difficult maintenance or operation, the time and attention required, and low cultural acceptability. In addition, chemical adsorbents have limited effectiveness in removing As[III], which makes up about 70-90\% of the total arsenic measured in Bangladeshi tubewells (Bhattacharya et al., 2002).

\subsection{A new implementation model for Bangladesh}

In light of the repeated problems with household filter adoption and the continuing prevalence of arsenic in drinking water in Bangladesh, researchers at LBNL have recognized the need to be innovative in implementation before deciding on technology design. This interdisciplinary approach has led to the development of a promising new implementation model for Bangladesh. In this model, an appropriate safe water technology is licensed to a community scale clean water center operating under a pubic-private partnership (PPP). Users pay a small fee to collect treated water from the local clean 
water center. This fee covers the capital and operating costs of treatment, the cost of arsenic monitoring, the capital costs of a small building, the salary of a few local employees, and a modest profit. A modest profit would provide an incentive for local stakeholders to efficiently maintain the system, tailor the technology to local conditions, monitor water quality, and upgrade the technology as appropriate. A community scale is advantageous because it (1) removes the burden of maintenance from the households, (2) allows for centralized monitoring of water quality, both ensuring that the treated water continues to be safe and allowing treatment to be tailored to local water conditions, lowering costs and waste, and (3) allows for rapid upgrades as new safer or lower cost technology is developed. Studies have also shown a strong preference for community scale treatment over household filters (Ahmad et al., 2003).

The PPP is a partnership between a private company, local financial institutions, a local NGO or social marketing company, and a local village government. The private company builds, operates, and maintains the center until the capital costs are paid off by the local government. The government can then choose to continue leasing operation and maintenance to the private company or choose some other provider. Participation by the financial institution allows for low upfront costs to the the local village government as well as startup equity for the private company. The private company contracts a local NGO or social marketing organization to provide local education and act as a community liason. Figure 1.1 shows a schematic detailing the roles, cash flow, and mutual benefit relationships between each of the partnering institutions in the PPP. The PPP model is an innovative way to balance the tradeoffs between public and private 
water treatment. Community participation is encouraged through the partnership with the local government and liaison activities provided by the local NGO. The benefits and viability of this model have been demonstrated in India by WaterHealth International (www.waterhealth.com). WaterHealth is a private company providing clean drinking water to more than a million people in rural Indian villages through publicly-owned, privately-managed village-scale water treatment centers operating under a PPP.

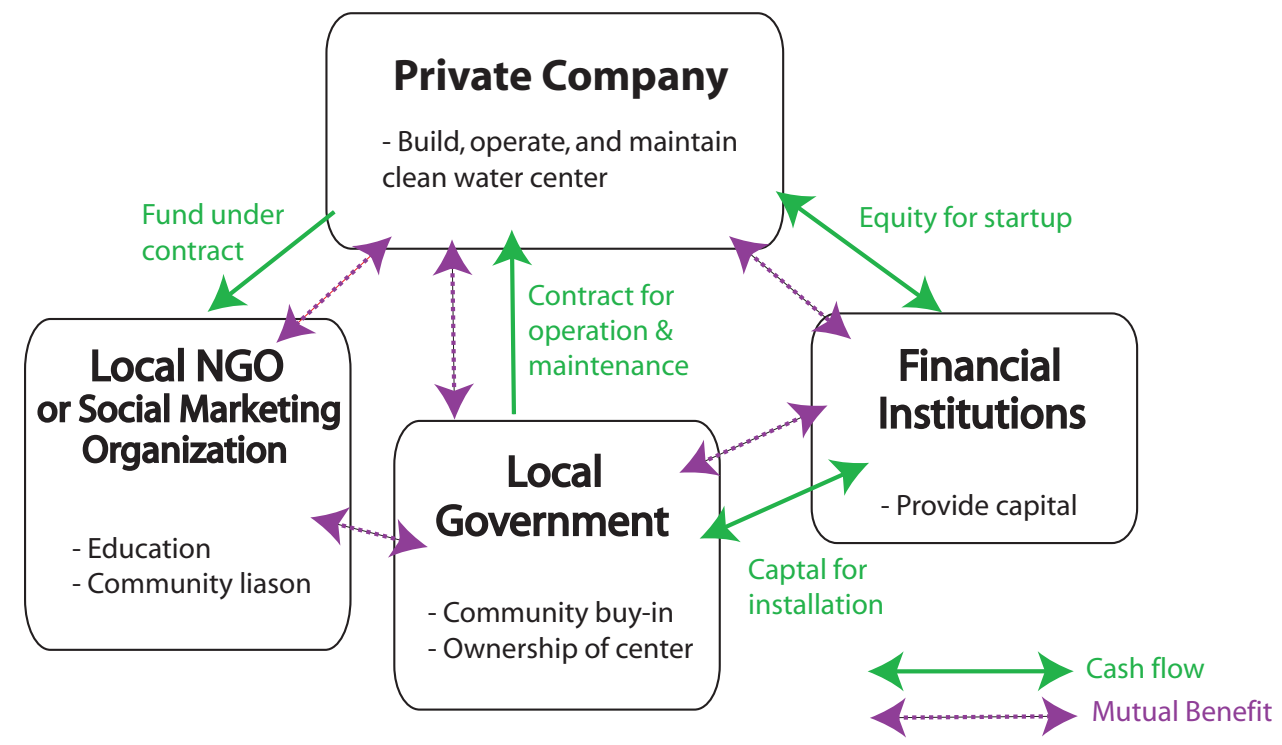

Figure 1.1: A schematic detailing the roles, cash flow, and mutual benefit relationships between each of the partnering institutions in a public-private partnership (PPP). The PPP is the key to long term viability of a community clean water center. Appropriate safe water technology would be licensed to the private company.

Safe water technologies must have certain qualities to be compatible with a community clean water center in rural Bangladesh. Operating costs must be extremely low for clean water to remain affordable to those making less than US $\$ 1$ per day. Using the model of WaterHealth, an ideal retail price would be $<$ US $\$ 0.025$ per 10 liters, constraining operating costs to be $<\$ 0.005$ per 10 liters. Waste output should 
be low, both for environmental protection and due to very stringent and costly waste disposal requirements for arsenic in Bangladesh (Mathieu et al., 2008). Maintenance and operation should require skills commensurate with a secondary education or less to take advantage of the local labor pool. Spare parts and tools for maintenance and repair should be available locally. Ideally, the system should be scalable, allowing for trialability in single village units followed by expansion. Last but not least, the technology should effectively reduce arsenic concentrations in Bangladesh to either the legal Bangladesh limit $(50 \mu \mathrm{g} / \mathrm{L})$ or ideally, the more conservative maximum recommended limit by the WHO $(10 \mu \mathrm{g} / \mathrm{L})$.

\subsection{ElectroChemical Arsenic Remediation (ECAR)}

The implementation model described above allows for a much wider array of technologies to be viable in Bangladesh than a household filter model. Specifically, an electricity-based technology could be viable if the treatment costs were low enough to offset the maintenance, operation, and capital costs of a small scale electricity source such as Photovoltaics (PV) or battery packs. In light of this possibility, a promising electricity-based technology known as ElectroChemical Arsenic Remediation (ECAR) has been designed for rural Bangladesh.

ECAR is a treatment method based heavily on electrocoagulation (EC; discussed in detail in Section 2.5.2). In EC (and ECAR), a sacrificial iron electrode is slowly electrochemically dissolved in arsenic-contaminated water. The dissolved iron quickly hydrolyzes, forming an iron (hydr)oxide sludge with a high affinity for arsenic. 
In ECAR, the iron (hydr)oxides are allowed further time to complex with arsenic and coagulate during post-electrolysis mixing (this is not usually the case in EC). Finally, arsenic-laden iron (hydr)oxides are separated from water, reducing the arsenic concentration to safe levels. For convenience, the iron (hydr)oxide sludge generated during ECAR is herein referred to as EGA (Electrochemically-Generated Adsorbent).

ECAR technology has a number of potential advantages over chemical adsorbents. EGA is manufactured at the time of use, eliminating the need for a costly adsorbent supply chain. In addition, electrochemical processes greatly enhance the arsenic removal capacity (i.e. arsenic removed per unit iron input) of ECAR relative to the chemical addition of ferric salts or metallic iron. This is thought to be due to (i) an increase in the rate of rust production (by factors of 10 to 100 over natural rusting rate of metallic iron), and (ii) the rapid electrochemical oxidation of As[III] to the more favorable As $[\mathrm{V}]$, which binds much more readily to iron-based adsorbents. Thus employing a small amount of electricity allows for a large increase in efficiency, lower operating costs and production of far less arsenic-laden waste than most chemical adsorbents. In addition, the electrodes are self-cleaning if current is alternated, reducing maintenance and eliminating the need to handle strong alkalies and corrosive acids for regeneration (required of activated alumina and other regenerative adsorbents). Iron, the only consumable input of ECAR (aside from electricity) is readily available in Bangladesh. Thus ECAR is a promising potential technology for a community clean water center in Bangladesh. 


\subsubsection{ECAR operating parameters}

The parameters controlling ECAR treatment include the current density, $j$ $\left(\mathrm{mA} / \mathrm{cm}^{2}\right)$, the charge density, $q(\mathrm{C} / \mathrm{L})$, and the duration of post-electrolysis mixing, $t_{M}$. Other parameters include the duration of filtration, $t_{f i l t}$ and the current processing time, $t_{C P} . t_{f i l t}$ is set by the filtration method and $t_{C P}$ is determined by $j$ and $q$ according to the following (derived in Section 2.5.2):

$$
t_{C P}=\frac{q}{j(A / V)}
$$

where $t_{C P}$ is the current processing time, $q$ is the charge density, $j$ is the current density, and $A / V$ is the ratio of electrode surface area to treatment volume. Each of the controlling parameters influence arsenic removal in different, but intertwined ways. These are briefly laid out below and more fully explained in Section 2.5.2.

Current density, $j$, controls the interface potential between the iron electrode and electrolyte (in this case contaminated groundwater). At a given $\mathrm{pH}$, the products of iron corrosion are highly dependent on the local potential, and thus $j$ influences the specific iron (hydr)oxide composition of EGA. The specific composition of EGA as a function of current density is unknown. In addition, the affinity of many iron (hydr)oxide species for arsenic is unknown. Differences in arsenic affinity could lead to certain regions of current density that generate more of less efficient EGA.

Charge density, $q$, describes the net charge added per liter of contaminated water. Charge can be converted to iron dosage using Faraday's equation and current efficiency measurements (Equation 2.19 and 2.21 respectively). Effectively, charge density 
measures the applied dosage of EGA.

The mixing time, $t_{M}$, controls the additional duration of contact between arsenic and EGA, allowing for further complexation and coagulation with existing EGA. Adding a separate mixing step allows ECAR to separate the effects of arsenic-EGA contact, which occurs during both dosing and mixing, from the effects of additional EGA dosage, which occurs during dosing.

\subsubsection{Other considerations - treatment time and cost}

Each of the ECAR parameters affects important performance considerations, such as treatment time and operating cost. The total treatment time is $t_{\text {treat }}=t_{C P}+$ $t_{M}+t_{f i l t} \cdot t_{C P}$ is set by $q$ and $j$ according to Equation 1.1, thus the total treatment time is a function of $j$ and $q$ in addition to $t_{f i l t}$ and $t_{M}$. A household system would require a short treatment time $(<1-2$ hours $)$ to compete with currently available filters. A community treatment center could effectively store water, extending the viable treatment time to days. However, given the increased potential for bacterial contamination with storage time, as well as a desire to prevent unpopular startup delays after maintenance or unforeseen shut downs, nighttime $(<12$ hour $)$ treatment would be advantageous. Notably, in rural areas, electricity is likely to be intermittent (due to line or photovoltaic

power supply patterns). Thus there is little to gain from decreasing treatment time to $<5-6$ hours, for the water will have to be stored anyway.

Operating costs depend on the amount of energy and iron required to reduce arsenic in Bangladesh groundwater to safe levels. Iron consumption is directly related 
to the charge density by Faraday's law (Equation 2.19). Thus the iron required will be based on the charge density required to reduce arsenic to safe levels. The charge density required is based on the affinity of EGA to complex with arsenic and then to coagulate into particles large enough to be filtered out. Since $j$ and $t_{M}$ affect the composition and extent of complexation and/or coagulation of EGA, both can indirectly affect the amount of charge density, and hence iron, required to produce safe arsenic levels. The energy requirement has similar dependencies. Energy is directly related to the amount of charge required by ECAR along with the operating voltage ${ }^{4}$. Thus energy consumption, as with iron, may be affected by $j$ and $t_{M}$. The dependancy on operating voltage means that energy consumption also depends on the resistivity of the contaminated water.

It is clear that each of the controllable ECAR operating parameters $(j, q$, and $\left.t_{M}\right)$ may affect each of the performance characteristics relevant for viable, effective, and low-cost arsenic removal in rural Bangladesh.

\subsubsection{Previous electrocoagulation research}

Previous electrocoagulation research has focused heavily on wastewater treatment. Electricity was first used to treat water in the UK in 1889 (Chen, 2004). In 1909, Electrocoagulation (EC) using aluminum and iron electrodes was patented in the US as a method for purifying wastewater. Electrolysis sludge treatment plants were in operation as early as 1911 in California and Oklahoma (Collier, 1912), though all plants were abandoned thirty years later due to rising costs (Vik et al., 1984). Since then, EC

\footnotetext{
${ }^{4}$ Note that ECAR runs under constant current conditions, so the operating voltage is not constant and will tend to drift slightly.
} 
has received new interest for different applications due to benefits such as environmental compatibility, versatility, energy efficiency, safety, selectivity, amenability to automation, and cost effectiveness (Mollah et al., 2004). EC has been used to treat wastewater containing heavy metals (Pogrebnaya et al., 1995), foodstuff (Chen et al., 2000; Beck et al., 1974), oil wastes (Biswas \& Lazarescu, 1991)[10], textile dyes (Vlyssides et al., 2000; Do \& Chen, 1994; Ibanez et al., 1998; Vlyssides et al., 1999; Gurses et al., 2002; Xiong et al., 2001), fluorine (Mameri et al., 1998), polymeric wastes (Panizza et al., 2000), organic matter from landfill leachate (Tsai et al., 1997), suspended particles (Szynkarczuk et al., 1994; Abuzaid et al., 2002), chemical and mechanical polishing wastes (Belongia et al., 1999), aqueous suspensions of ultrafine particles (Matteson et al., 1995), nitrate (Koparal \& Ogutveren, 2002; Mishra, 2006), phenolic waste (Phutdhawong et al., 2000), and refractory organic pollutants including lignin and EDTA (Pouet \& Grasmick, 1995; Chiang et al., 1997; Chen, 2004). Recently, EC has also been proposed to treat potable water for humus removal, color, and disinfection (Vik et al., 1984; Pouet \& Grasmick, 1995; Chen, 2004; Holt et al., 2002).

Proposing EC to reduce arsenic concentrations in water is relatively new. Much of the focus has been on removing high levels of arsenic (1-1000 mg/L) from wastewater, usually at low pH (Balasubramanian \& Madhavan, 2001; Pinisakul et al., 2002; Mishra, 2006; Hansen et al., 2006; Gomes et al., 2007). Fewer studies have looked at arsenic removal in potable water at neutral pH(Parga et al., 2005a; Kumar et al., 2004; Gomes et al., 2007; Arienzo et al., 2002). These studies are discussed in more detail in Section 2.5.5. 
The majority of the above studies concentrate on the empirical optimization. The influence of parameters $j$ and $q$ on removal efficiency in EC have been studied to a small extent with mixed results. Chen et al. (2000) and Kumar et al. (2004) both reported that charge density alone (not current density) governs EC efficiency in current density ranges $j=3-30 \mathrm{~mA} / \mathrm{cm}^{2}$ and $j=0.65$ and 1.53 macmsq respectively, though current density was found to have a strong influence on the treatment time. Pouet \& Grasmick (1995) reported that current density can effect treatment efficiency, though Chen et al. (2000) attribute these results to a changing charge density across experiments.

\subsubsection{The need for parameter studies in Bangladesh groundwater}

Of the experiments to study the influence of $j$ and $q$, only Kumar et al. (2004) used arsenic removal at neutral $\mathrm{pH}$ as a metric to study EC. The group reported effective removal of $\mathrm{As}[\mathrm{III}]$ and $\mathrm{As}[\mathrm{V}]$, reducing arsenic-spiked Indian tap water from $2 \mathrm{mg} / \mathrm{L}$ to below the WHO limit of $10 \mu \mathrm{g} / \mathrm{L}$ at a charge density of approximately $100 \mathrm{C} / \mathrm{L}$. However, the water contained no detectable phosphate and low bicarbonate compared to Bangladesh groundwater (silicate not measured). These ions, all present in abundance in Bangladesh (BGS, 2001), are known to have a highly detrimental effect on arsenic removal using iron (hydr)oxides (described in detail in Section 2.4.6). Prior to this work, no researcher to the authors knowledge has studied the influence of ECAR (or EC) operating parameters on arsenic removal in the specific groundwater environment of Bangladesh. Such studies are needed to determine both (1) if ECAR can operate viably,

effectively, and cheaply in the groundwater of Bangladesh and (2) how the parameters 
might be used to optimize ECAR in the field. To answer each of these questions, it is necessary to perform both empirical studies (i.e. study the arsenic removal response at various parameter combinations) and exploratory studies to help uncover, and therefore control, the underlying arsenic removal mechanism in ECAR.

\subsection{Thesis objectives and dissertation structure}

Electrochemical arsenic remediation (ECAR) is a promising technology that is compatible with a community clean water center implementation model and has the potential to operate at extremely low cost. This thesis presents research into the viability and effectiveness of ECAR as a low-cost treatment technology for rural Bangladesh.

As an overall theme, this research seeks to demonstrate arsenic removal effectiveness of ECAR in the groundwater environment of Bangladesh and explore the influence of several ECAR operating parameters on arsenic removal capacity (i.e. arsenic removed per coulomb) acting as a predictor of operating costs. Understanding the mechanism of arsenic removal as a function of current density is highlighted as potential means of further optimization. The cause of current density trends is explored using in-situ techniques (transient voltammetry and EIS) and analysis of reaction products (via chemical analysis, scanning electron microscopy (SEM), X-ray diffraction (XRD), and X-ray absorption spectroscopy (XAS)).

Chapters 2, 3, 4, and 5 of this thesis are summarized below. The motivating ideas for each chapter are given along with a list of specific research objectives (for Chapters 3, 4, and 5). Chapter 6 provides a summary. 


\subsubsection{Chapter 2: Relevant Scientific Background}

This chapter contains a review of key scientific concepts and literature used to inform the scientific analysis in chapters to come. Basic properties of arsenic and iron (hydr)oxides are given, followed by a discussion of sorption and adsorption and a review of arsenic adsorption onto iron (hydr)oxides in the literature. Some basic electrochemistry is reviewed, followed by a detailed description of the theory of electrocoagulation and a review of studies using electrocoagulation to remove arsenic from water.

\subsubsection{Chapter 3: Chemical and Physical Analysis of Arsenic Complex- ation with Iron in ECAR}

The parameters in ECAR - current density, $j$, charge density, $q$, current processing time, $t_{C P}$, mixing time, $t_{M}$, and filtration time, $t_{f i l t}$ - may have significant and potentially competing influences on performance considerations relevant to rural Bangladesh - arsenic removal effectiveness, treatment time, and operating costs. To date, only one study has looked at the influence of operating parameters in electrocoagulation on arsenic removal efficiency at neutral pH (Kumar et al., 2004). These experiments were performed in spiked tap water that differs significantly in composition from groundwater in Bangladesh. In addition, only a small range of current density was explored (0.65 $1.53 \mathrm{~mA} / \mathrm{cm}^{2}$ ), ignoring more extreme regions that could produce higher arsenic removal efficiency. No parameters other than $j$ and $q$ were varied for iron electrodes.

To better understand the influences of ECAR parameters on performance criteria in rural Bangladesh, empirical studies must be performed in the specific groundwater 
environment of Bangladesh. Exploratory studies to understand the cause of parameter influence are also essential to better predict and optimize ECAR performance, specifically with regard to lowering costs (a key consideration for rural Bangladesh).

\section{Chapter 3 Research Objectives}

1. Design a laboratory method for ECAR keeping in mind eventual scale-up.

2. Demonstrate the capability of ECAR to reduce high levels of arsenic (>98\% surveyed wells) to below the WHO recommended maximum limit of $10 \mu \mathrm{g} / \mathrm{L}$ in synthetic Bangladesh groundwater constituted to mimic key properties relevant to arsenic removal.

3. Characterize arsenic removal capacity of ECAR with respect to current density, $j$, and charge density, $q$, in synthetic Bangladesh groundwater.

4. Explore the cause of current density effects of arsenic removal capacity and the mechanism of As[III] removal.

5. Identify trends and tradeoffs in removal capacity and treatment time between operating parameters.

6. Identify an optimal range of current density and charge density for Bangladesh taking into account arsenic removal effectiveness, treatment time, and minimization of operating costs.

7. Explore low cost alternatives to membrane filtration in ECAR. 


\subsubsection{Chapter 4: Characterization of Reaction Products}

The ECAR process produces arsenic-laden iron (hydr)oxides sludge (i.e. EGA) as a waste byproduct. The amount and chemical composition of the waste are key to understanding safe disposal mechanisms and potential disposal costs in Bangladesh. The sludge can also be analyzed to look for changes in the chemical structure of the EGA produced at different current densities as well as the chemical structure of arsenic sorbed to EGA X-ray absorption spectroscopy. This can be used to compare the arsenic removal mechanism at different current densities and understand the cause of trends in arsenic removal capacity with current density.

The particle size of EGA sludge was also explored using scanning electron microscopy (SEM) and membrane pore size experiments. The particle size is a critical parameter for determining an appropriate low-cost filtration or sedimentation method for Bangladesh.

\section{Chapter 4 Research Objectives}

1. Measure the amount and Fe/As ratio of EGA sludge produced in ECAR at various current densities.

2. Estimate the EGA particle size using SEM and break through with membranes of different pore size.

3. Compare the iron (hydr)oxides structure of EGA produced at different current densities (EXAFS). 
4. Compare the arsenic-iron (hydr)oxides structure in arsenic-laden EGA at different current densities (EXAFS).

5. Compare the iron (hydr)oxides structure of EGA to known reference iron oxyhydroxide and iron hydroxide compounds (EXAFS).

6. Compare the arsenic-iron (hydr)oxides structure in arsenic-laden EGA to the known structure of arsenic adsorbed to various iron (hydr)oxides and scorodite (EXAFS).

\subsubsection{Chapter 5: ECAR Performance in Real Bangladesh Groundwa- ter}

Neither ECAR nor EC has ever been tested in real Bangladesh groundwater. Actual groundwater contains a wide range of co-occuring solutes in addition to arsenic that have been known to affect arsenic removal using iron (hydr)oxides. Thus, it is essential to demonstrate the ability of ECAR to remove arsenic from real arsenic-contaminated groundwater from tube well sources in Bangladesh. Since a large sample size is not feasible in the scope of this thesis, some analysis of co-occuring solutes in real groundwater is necessary to characterize the representativeness of a small set of samples in the context of all contaminated Bangladesh groundwater.

In addition, synthetic Bangladesh groundwater spiked with both As[III] and $\mathrm{As}[\mathrm{V}]$ showed much higher than expected oxidation rates of As[III] to As[V] during storage. If this effect is present in real groundwater, then storage might be an effective way to convert high As[III] concentrations to As[V]. Although As[III] oxidation is expected to be a byproduct of electrochemical processes in the cell, it is not known if this process 
is thermodynamically or kinetically favorable at all ECAR operating conditions, thus backup options are desirable. The opportunity to sample real Bangladesh groundwater was leveraged to test for rapid oxidation of As[III] during groundwater storage.

\section{Chapter 5 Research Objectives}

1. Demonstrate the ability of ECAR treatment to reduce arsenic levels from 100 $500 \mu \mathrm{g} / \mathrm{L}$ in real Bangladesh groundwater to below either the Bangladesh legal limit $(50 \mu \mathrm{g} / \mathrm{L})$ or ideally, the WHO recommended maximum limit $(10 \mu \mathrm{g} / \mathrm{L})$.

2. Characterize the co-occurring solute composition of tube well water samples and compare to regional and national survey data from BGS (2001).

3. Measure the level of $\mathrm{As}[\mathrm{III}]$ to $\mathrm{As}[\mathrm{V}]$ oxidation in tube well water samples up to seven days after collection. 


\section{Chapter 2}

\section{Relevant Scientific Background}

\section{$2.1 \quad$ Introduction}

This chapter contains a review of key scientific concepts and literature used to inform the scientific analysis in chapters to come. Basic properties of arsenic and iron (hydr)oxides are given, followed by a discussion of sorption and adsorption and a review of arsenic adsorption onto iron (hydr)oxides in the literature. Some basic electrochemistry is reviewed, followed by a detailed description of the theory of electrocoagulation and a review of studies using electrocoagulation to remove arsenic from water.

\subsection{Arsenic in Natural Waters}

Arsenic forms a number of inorganic compounds, including hydrides (e.g. arsine), halides, oxides, acids, and sulfides. Of greater than 320 known arsenic minerals, fewer than 10 are commonly identified in the environment (Welch et al., 1988) - the 
most commonly reported (excepting scorodite) are arsenic oxides and arsenic sulfides. Arsenic is very sensitive to mobilization in the typical $\mathrm{pH}$ range of groundwaters $(\mathrm{pH}$ 6.5-8.5) under both oxidizing and reducing conditions, making it unique among heavy metalloids and oxyanion-forming elements (e.g. As, Se, Sb, Mo, V, Cr, U, Re)(Smedley \& Kinniburgh, 2002). Arsenic can occur in the environment in the $-3,0,+3$, and +5 oxidation state, though in natural waters it is mostly found as oxyanions of trivalent arsenite $[\mathrm{As}[\mathrm{III}]]$ or pentavalent arsenate $[\mathrm{As}[\mathrm{V}]]$. The main difference between the two is that arsenate has an oxygen double bond in the molecular structure. In oxygenated waters, arsenic acid species $\left(\mathrm{H}_{3} \mathrm{AsO}_{4}, \mathrm{H}_{2} \mathrm{AsO}_{4}^{-}, \mathrm{HAsO}_{4}^{2-}\right.$, and $\left.\mathrm{AsO}_{4}^{3-}\right)$ are stable, with $\mathrm{H}_{2} \mathrm{AsO}_{4}^{-}$dominating at low $\mathrm{pH}(\mathrm{pH}<6.9)$ and $\mathrm{HAsO}_{4}^{2-}$ at higher $\mathrm{pH}\left(\mathrm{H}_{3} \mathrm{AsO}_{4}\right.$ and $\mathrm{AsO}_{4}^{3-}$ occur in extremely acidic and alkaline conditions respectively). In mild reducing conditions, arsenious acid species $\left(\mathrm{H}_{3} \mathrm{AsO}_{3}, \mathrm{H}_{2} \mathrm{AsO}_{3}^{-}\right.$, and $\left.\mathrm{HAsO}_{3}^{2-}\right)$ become stable, with the uncharged species $\mathrm{H}_{3} \mathrm{AsO}_{3}$ dominating at a $\mathrm{pH}$ less than about $\mathrm{pH}$ 9.2. Organic arsenic forms may be produced by biological activity, but this mostly occurs in surface waters and very rarely in areas significantly impacted by industrial pollution (Smedley \& Kinniburgh, 2002).

In groundwaters, the ratio of $\mathrm{As}[\mathrm{III}]$ to $\mathrm{As}[\mathrm{V}]$ can vary greatly depending on variations in the abundance of redox-active solids, especially organic carbon, the activity of microorganisms, and the extent of convection and diffusion of $\mathrm{O}_{2}$ from the atmosphere (Smedley \& Kinniburgh, 2002). Equilibrium calculations predict that As[V] concentrations should be greater than As[III] concentrations in all but strongly reducing conditions - however this is not always found to be the case in natural waters. In practice, redox 
equilibrium is achieved slowly and the redox potential tends to be controlled by the major elements (O, C, N, S, and Fe) (Smedley \& Kinniburgh, 2002). Thus in many areas, including the groundwater of Bangladesh, As[III] has been found under oxidizing conditions (BGS, 2001; Abdullah et al., 1995). As[III] is more toxic to humans (Korte \& Fernando, 1991; Balasubramanian \& Madhavan, 2001; Yamauchi \& Fowler, 1994) and tends to be more difficult to remove from water (see section 2.4).

Most toxic trace metals exist in solution as cations (e.g. $\mathrm{Pb}^{2+}, \mathrm{Cu}^{2+}, \mathrm{Ni}^{2+}$,

$\left.\mathrm{Cd}^{2+}, \mathrm{Co}^{2+}, \mathrm{Zn}^{2+}\right)$. These generally become increasingly insoluble as the $\mathrm{pH}$ increases from acidic values, becoming less mobile at the near-neutral $\mathrm{pH}$ typical of most groundwaters. In contrast, most oxyanions, including arsenate, tend to become more mobile as the $\mathrm{pH}$ increases from acidic values because mobility is often determined by sorption strength rather than solubility (Dzombak \& Morel, 1990). This allows anions to persist in solution in relatively high concentrations even at near-neutral pH. For this reason, the oxyanion forming elements such as Cr, As, U and Se are some of the most common trace contaminants in groundwaters (Smedley \& Kinniburgh, 2002).

\subsection{Iron (Hydr)oxides}

Iron (hydr)oxides are ubiquitous compounds in nature and easy to synthesize through industrial processes and in the laboratory. There are 16 iron (hydr)oxides (Table 2.1 ), comprised of iron oxides, iron hydroxides, and iron oxyhydroxides (also known as oxide-hydroxides) - collectively referred to in this work as iron (hydr)oxides. In addition, there are numerous non-stoichiometric, or mixed, states. All iron (hydr)oxides are 
composed of Fe together with $\mathrm{O}$ and/or $\mathrm{OH}$, with $\mathrm{Fe}$ in the trivalent (most common) or divalent state. The various (hydr)oxides differ in arrangement of basic structural units $\mathrm{Fe}(\mathrm{O}, \mathrm{OH})_{6}$ or $\mathrm{Fe}(\mathrm{O})_{4}$ - in space. These basic polyhedra can be linked by faces, edges, or corners. The combination of linkages form the basis of the iron (hydr)oxides mineralogical structure, and result in different characteristic Fe-Fe interatomic differences (Toner et al., 2008). In some cases, small amounts of anions such as $\mathrm{Cl}^{-}, \mathrm{SO}_{4}^{2-}$, or $\mathrm{CO}_{3}^{2-}$ may also participate in the structure.

The oxyhydroxides can be dehydroxylated to their oxide counterparts. This is due in part to the similarity between anion frameworks - often only the rearrangement of cations and the loss of $\mathrm{OH}$ are required to effect a transformation (Cornell \& Schwertmann, 2000). The Fe[III] (hydr)oxides exhibit low solubility (leading to high stability) and brilliant colors, often in orange to red shades. Due to their high energy of crystallization, iron (hydr)oxides often form minute crystals with a high specific surface area, often $>100 \mathrm{~m}^{2} / \mathrm{g}$ (Cornell \& Schwertmann, 2000), making them effective sorbents for a large range of dissolved ions, molecules, and gases.

Iron oxide minerals tend to form in poorly crystalline states with high specific surface area such as amorphous-Fe( $\mathrm{OH})_{3}$ but, over time, undergo transformations to more crystalline forms such as goethite or hematite (Dixit \& Hering, 2003; Benjamin, 2002). The time scale of the transformation can depend on temperature, $\mathrm{pH}$, and the presence of co-occuring solutes (Cornell \& Schwertmann, 2000; Ford, 2002).

The following is a brief description of the compounds listed in Table 2.1, based heavily on Cornell \& Schwertmann (2000). More detailed descriptions (along with an 
excellent treatment of many iron (hydr)oxides properties) can be found in Cornell \& Schwertmann (2000).

Goethite, $\alpha$-FeOOH, occurs in rocks and is one of the most thermodynamically stable iron (hydr)oxides at ambient temperatures, making it either the first (hydr)oxide to form, or the end member of many transformations. As a powder, Goethite is yellow. Its structure is based on hexagonal close packing of anions.

Lepidocrocite, $\gamma$-FeOOH, is orange colored and occurs in rocks, soils, biota, and rust and is often an oxidation product of $\mathrm{Fe}^{2+}$. Its structure is based on cubic close packing of anions.

Akaganeite, $\beta-\mathrm{FeOOH}$, occurs rarely in nature and has a structure based on body centered cubic packing of anions, often containing a low level of either chloride or fluoride. It appears brown to bright yellow.

Schwertmannite, $\mathrm{Fe}_{16} \mathrm{O}_{16}(\mathrm{OH})_{\mathrm{y}}\left(\mathrm{SO}_{4}\right)_{\mathrm{z}} \cdot n \mathrm{H}_{2} \mathrm{O}$, has the same basic structure as akaganeite, but contains sulphate instead of chloride ions. It frequently occurs in nature as an oxidation product of pyrite, but is unlikely to play a role in this work due to the relatively low concentrations of sulphate in the environment of interest.

$\delta$-FeOOH (synthetic) and its poorly crystalline mineral form Feroxyhyte, $\gamma /$-FeOOH, are reddish-brown and ferrimagnetic with hexagonal close packing of anions. Feroxyhyte is very rare, but found in certain surface environments.

Ferrihydrite, $\mathrm{Fe}_{5} \mathrm{HO}_{8} \cdot 4 \mathrm{H}_{2} \mathrm{O}$, is reddish-brown and widespread across many surface environments. Ferrihydrite is often termed "amorphous iron oxide" or "hydrous 
ferric oxide (HFO)." Unlike other iron (hydr)oxides, it exists exclusively as nanocrystals and, unless stabilized in some way, transforms with time into more stable and more crystalline iron (hydr)oxides. It is an important precursor, and thus very important for this work. Structurally, it consists of hexagonal closely packed anions and is a mixture of defect-free and defective structural units. The composition, especially with respect to $\mathrm{OH}$ and $\mathrm{H}_{2} \mathrm{O}$, seems to be variable - thus the formula $\mathrm{Fe}_{5} \mathrm{HO}_{8} \cdot 4 \mathrm{H}_{2} \mathrm{O}$ is often used as a preliminary starting point.

Bernalite, $\mathrm{Fe}(\mathrm{OH})_{3}$, is a greenish hydroxide that to date, has been found only in two locations (Birch et al., 1993; Kolitsch, 1998).

$\mathrm{Fe}(\mathrm{OH})_{2}$ does not exist as a mineral, but is readily oxidized, developing into greenish blue green rust, or black magnetite.

Hematite, $\alpha-\mathrm{Fe}_{2} \mathrm{O}_{3}$, is the oldest known iron (hydr)oxides mineral and is found abundantly in rocks and soils. It is blood-red if finely divided, or black to grey if coarsely crystalline. Its structure is based on hexagonal close packing of anions, and, like Goethite, it is extremely stable and often the end member of transformations of other iron oxides. Hematite is also known as iron ${ }^{\mathrm{III}}$ oxide and ferric oxide.

Magnetite, $\mathrm{Fe}_{3} \mathrm{O}_{4}$, is a black, ferrimagnetic mineral containing both $\mathrm{Fe}[\mathrm{II}]$ and $\mathrm{Fe}[\mathrm{III}]$ with an inverse spinel structure. It is the principal component in loadstones, the earliest known permanent magnets.

Maghemite, $\gamma-\mathrm{Fe}_{2} \mathrm{O}_{3}$, is a red-brown, ferrimagnetic mineral with nearly the same structure as magnetite, but containing cation deficient sites. It occurs in soils as a 
Table 2.1: The iron (hydr)oxides. Adapted from Cornell \& Schwertmann (2000)

\begin{tabular}{|c|c|}
\hline Oxyhydroxides and Hydroxides & Oxides \\
\hline $\begin{array}{l}\text { Goethite } \alpha \text {-FeOOH } \\
\text { Lepidocrocite } \gamma \text {-FeOOH } \\
\text { Akaganeite } \beta \text {-FeOOH } \\
\text { Schwertmannite } \mathrm{Fe}_{16} \mathrm{O}_{16}(\mathrm{OH})_{\mathrm{y}}\left(\mathrm{SO}_{4}\right)_{\mathrm{z}} \cdot n \mathrm{H}_{2} \mathrm{O} \\
\delta \text {-FeOOH } \\
\text { Feroxyhyte } \gamma / \text {-FeOOH } \\
\text { High Pressure } \mathrm{FeOOH} \\
\text { Ferrihydrite } \mathrm{Fe}_{5} \mathrm{HO}_{8} \cdot 4 \mathrm{H}_{2} \mathrm{O} \\
\text { Bernalite } \mathrm{Fe}(\mathrm{OH})_{3} \\
\mathrm{Fe}(\mathrm{OH})_{2} \\
\text { Green Rusts } \mathrm{Fe}_{\mathrm{x}}^{\mathrm{III}} \mathrm{Fe}_{\mathrm{y}}^{\mathrm{II}}(\mathrm{OH})_{3 \mathrm{x}+2 \mathrm{y}-\mathrm{z}}\left(\mathrm{A}^{-}\right)_{\mathrm{z} ;} \mathrm{A}^{-}=\mathrm{Cl}_{;}^{-}\left(\frac{1}{2}\right) \mathrm{SO}_{4}^{2-}\end{array}$ & $\begin{array}{l}\text { Hematite } \alpha-\mathrm{Fe}_{2} \mathrm{O}_{3} \\
\text { Magnetite } \mathrm{Fe}_{3} \mathrm{O}_{4}\left(\mathrm{Fe}^{\mathrm{II}} \mathrm{Fe}_{2} \mathrm{III} \mathrm{O}_{4}\right) \\
\text { Maghemite } \gamma-\mathrm{Fe}_{2} \mathrm{O}_{3} \\
\beta-\mathrm{Fe}_{2} \mathrm{O}_{3} \\
\varepsilon-\mathrm{Fe}_{2} \mathrm{O}_{3} \\
\text { Wüstite } \mathrm{FeO}\end{array}$ \\
\hline
\end{tabular}

weathering product of magnetite or a product of heating other iron (hydr)oxides.

$\beta-\mathrm{Fe}_{2} \mathrm{O}_{3}, \varepsilon-\mathrm{Fe}_{2} \mathrm{O}_{3}$, and $\mathrm{High}$ Pressure $\mathrm{FeOOH}$ are rare laboratory compounds obtained only in high vacuum, high temperature $\left(170-750{ }^{\circ} \mathrm{C}\right)$, and/or high pressure $(8$ Gpa).

Wüstite, FeO, is black, contains only divalent Fe, and is usually non-stoichiometric (Odeficient). The structure is similar to $\mathrm{NaCl}$ and is based on cubic close packing of anions.

Green Rusts consist of layers of $\mathrm{Fe}^{\mathrm{II}} \mathrm{OH}$ octahedra in which some $\mathrm{Fe}[\mathrm{II}]$ is replaced by $\mathrm{F}(\mathrm{III})$ and, to maintain neutrality, anions suh as $\mathrm{Cl}^{-}$and $\mathrm{SO}_{4}^{2-}$ are bound between the layers. Green rusts commonly form as corrosion products. 


\subsection{Arsenic sorption onto iron (hydr)oxides}

\subsubsection{Theory}

Adsorption is the accumulation of a substance at or near an interface relative to its concentration in the bulk solution (Benjamin, 2002), also called surface complexation. The substance that adsorbs is called the adsorbate, and the solid to which it binds is called the adsorbent. Although adsorption is technically distinct from absorption, which is the accumulation of substance in the interior of a non-aqueous phase, the two processes are often referred to together using the terms sorption, sorbate, and sorbent. Sorption can also include surface precipitation (Sposito, 1986), which can occur when the accumulation of adsorbate at the surface is sufficient for a new iron-arsenic phase to precipitate on the surface of the iron (hydr)oxide (i.e. a regular stoichiometric crystallike structure that can grow as opposed to a single surface layer of adsorbed ions). Distinct from all of the above is the formation of a solid-solution phase, such as ferric arsenate $\left(\mathrm{FeAsO}_{4}\right)$, in which arsenic is incorporated into the bulk phase rather than just the surface. For this work, sorption will refer to a combination of adsorption, absorption and/or surface precipitation. The term adsorption will be reserved for surface

complexation only, distinct from surface precipitation. Solid-solution formation will be referred to independently.

The mechanism of adsorption is often chemical interactions between the adsorbate and surface rather than electrostatic interactions. When this is the case, the adsorbate is said to be chemically or specifically adsorbed (often called chemisorbed). If 
the adsorbate is attracted to the surface solely through electrostatic interactions, then it is said to be physically or non-specifically adsorbed. The interaction between dissolved arsenic oxyanions and iron (hydr)oxides occurs at the iron (hydr)oxide surface. In the presence of water, the surface of an oxide is assumed to be hydrated and covered with a layer of $\mathrm{OH}_{\mathrm{x}}$ groups (either from the oxide structure or bound water molecules). Functional hydroxyl groups on a surface contain the same donor atoms as functional groups in soluble ligands, as so behave in the same way. The groups contain ionizable atoms and so can protonate and deprotonate as a function of $\mathrm{pH}$ (i.e. acid/base reactions). In the case of arsenic oxyanions, the main mechanism of adsorption is ligand exchange (Stollenwerk, 2003); the surface hydroxyl group is exchanged for another ligand. Ligand exchange can occur at one or two surface hydroxyl groups on the oxide, designated by $\mathrm{S}-\mathrm{OH}$, according to the following:

$$
\begin{gathered}
\mathrm{S}-\mathrm{OH}+\mathrm{L} \rightleftharpoons \mathrm{S}-\mathrm{L}^{+}+\mathrm{OH}^{-} \\
2 \mathrm{~S}-\mathrm{OH}+\mathrm{L} \rightleftharpoons \mathrm{S}_{2} \mathrm{~L}^{2+}+2 \mathrm{OH}^{-}
\end{gathered}
$$

These bonds are strong and are known as inner-sphere complexes. If the bond occurs at one hydroxyl group (Equation 2.1), it is known a monodentate complex. If it occurs at two hydroxyl groups (Equation 2.2), it is a bidentate complex. Since adsorption is coupled with the release of $\mathrm{OH}$ - in both cases, complexation tends to be favored by lower $\mathrm{pH}$ values. A complex in which the adsorbate retains its waters of hydration (while still engaging in a chemical bond) is known as an outer-sphere complex.

The capacity of a suspended solid surface adsorbent to adsorb a species of ion from solution depends on many factors, including the number of available sites on its 
surface, the affinity of adsorbate for each site, as well as on the amount of adsorbate available near the surface. The availability of sites on the adsorbent depends on the adsorbent surface area, the protonation state of the surface hydroxyl groups (which depends on $\mathrm{pH}$ ), and the concentration of competing ions for the same sites. The affinity of the adsorbate for a site depends on local electrostatic conditions (partially created by the protonation state of the surface hydroxyl groups) as well as the thermodynamic favorability of a chemical reaction. Each of these factors can limit and/or control the quantity of adsorbate removed from the solution through adsorption.

The amount of material adsorbed per unit amount of adsorbent is called the adsorption density. Any model that relates the amount of adsorbate at the surface to that in the solution in equilibrium systems at constant temperature is called an adsorption isotherm. Adsorption of $\mathrm{As}[\mathrm{III}]$ and $\mathrm{As}[\mathrm{V}]$ to iron oxides and iron hydroxides can be described by a Langmuir isotherm (Ferguson \& Gavis, 1972; Anderson et al., 1976; Gupta \& Chen, 1978; Pierce \& Moore, 1982; Sun \& Doner, 1996). The form of the Langmuir isotherm for a simple system with a single adsorbate is:

$$
q_{A}=\frac{K_{a d s}\{A\}}{1+K_{a d s}\{A\}} q_{\max }
$$

$$
\begin{aligned}
& q_{A}=\text { adsorption density (moles adsorbed per gram of adsorbent) } \\
& K_{a d s}=\text { constant defining affinity of adsorbent for adsorbate } \\
& \{A\}=\text { activity } \times \text { aqueous concentration of adsorbate } A \\
& q_{\max }=\text { maximum adsorption density. }
\end{aligned}
$$

When $K_{a d s}\{A\}<<1$, the isotherm becomes linear: $q_{A} \approx K_{a d s}\{A\} q_{\max }$. Over this limited range of adsorbate concentrations, one can expect the percentage of adsorbate 
removed from solution to be approximately constant. As $K_{a d s}\{A\}>>1, q_{A} \approx q_{\max }$, and the percentage of adsorbate removed will decrease will increasing adsorbate concentration.

If multiple adsorbates are in solution, they may compete for adsorption sites on a single adsorbent. In the case of $j$ adsorbates, the Langmuir adsorption isotherm for any species $i$ becomes:

$$
q_{i}=\frac{K_{a d s, i} a_{i}}{1+\sum_{j} K_{a d s, j} a_{j}} q_{\max }
$$

$K_{a d s, i}=$ constant defining affinity of adsorbent for adsorbate $i$

$a_{i}=\{\mathrm{A}\}$ for adsorbate $i$

See Benjamin (2002), chapter 10, or Stumm \& Morgan (1996), chapter 9, for more details on the derivation and behavior of isotherms.

The surface of the adsorbent can acquire electric charge though chemical reactions via ionizable functional groups (i.e. acid/base reactions, dependant on $\mathrm{pH}$ ), isomorphic replacements of atoms in the surface lattice, and adsorption. The charge can affect the affinity of the adsorbent for the adsorbate. To take this into account, the constant $K_{a d s}$ can be modified by an exponential term that is a function of the surface potential (Benjamin, 2002; Stumm \& Morgan, 1996). In general, a more charged surface will have an increased affinity for opposite-charge adsorbates and a decreased affinity for same-charge adsorbates. Recall from Section 2.2 that the stable forms of arsenate between $\mathrm{pH}$ 6-9 are negatively charged $\left(\mathrm{H}_{2} \mathrm{AsO}_{4}^{2-}\right.$ and $\left.\mathrm{HAsO}_{4}^{2-}\right)$ while the stable form of arsenite is neutral $\left(\mathrm{H}_{3} \mathrm{AsO}_{3}^{0}\right)$. 
If the adsorption density becomes high enough, it is possible for the adsorbate to form a surface precipitate. In theory, this would eliminate the limiting capacity of the solid for adsorbate (i.e. adherence to Equations 2.3 and 2.4), since ions from the bulk could always (subject to solubility equilibrium) be incorporated into a new precipitate layer as opposed to competing for adsorption sites. This appears to provide a method for distinguishing adsorption from surface precipitation by measuring the isotherm behavior (a surface precipitate should have no $q_{\max }$ ). However, in practice it almost impossible to distinguish the two states from isotherms alone. Surface precipitation is likely to be accompanied by adsorption to other parts of the surface with the sum of both behaviors appearing in the isotherm. In addition, if the affinity of the adsorbent for the adsorbate varies across the surface (as it often does), the isotherm may appear to increase indefinitely as strong binding sites become saturated but weaker binding sites continue to come into play at higher adsorbate concentrations - the isotherms become nearly impossible to distinguish from the expected isotherm of surface precipitation. Sensitive spectroscopic techniques, such as X-ray Absorption Spectroscopy (XAS), are often used to clarify the ambiguity instead.

Sorption of arsenic in a lab setting is commonly achieved by either coprecipitation or post-synthesis adsorption (PSA). In coprecipitation, iron (hydr)oxides are formed in the presence of arsenic oxyanions. In PSA, iron (hydr)oxides are formed prior to the addition of arsenic. The adsorbent aging period before the arsenic is added allows some time for coagulation of the adsorbent and crystallite growth to occur, both processes which tend to decrease the number of adsorption sites (Waychunas et al., 1993). If, 
however, a sorbing ion is present in solution during the hydrolysis and precipitation of iron (hydr)oxides (as in coprecipitation), then the ion may sorb to surface sites before aggregates are formed. This maximizes the number of available surface sites, increasing the sorptive capacity of the iron (hydr)oxides (Fuller et al., 1993). For modeling of natural systems, coprecipitation is often more appropriate, since natural iron(III)hydroxides are often formed from oxidation of $\mathrm{Fe}^{2+}$ ions in the presence of arsenic (Roberts et al., 2004). However, numerous arsenic remediation technologies exist that are based on either method, and therefore both coprecipitation and PSA are commonly used in experimentation.

\subsubsection{Review of arsenic adsorption literature}

A number of iron (hydr)oxides adsorbents have been effectively used to remove arsenic compounds from water. These include pre-formed iron (hydr)oxides (Pierce \& Moore, 1982; Goldberg, 1986; Waychunas et al., 1993; Fuller et al., 1993; Fendorf et al., 1997; Grossl et al., 1997; Raven et al., 1998; Manning et al., 1998; Jain et al., 1999) and coprecipitation with ferric and ferrous salts (Hering et al., 1996; Roberts et al., 2004; Sorg \& Logsdon, 1978). Zero-valent iron (ZVI) filings have also been effective at removing arsenic compounds from water (Su \& Puls, 2001; Lackovic et al., 2000; Bang et al., 2005b,a), presumably due to adsorption onto iron corrosion products. In direct comparisons, coprecipitation has been found to be more efficient than arsenic adsorption onto pre-formed iron (hydr)oxides (Fuller et al., 1993; Edwards, 1994), referred to here as PSA. However, in addition to being more pronounced at high aorbate-sorbent ratios, 
these effects were found to be slowly reversible (Hering et al., 1996), with the excess As[V] gradually releasing back into solution until the adsorption density of coprecipitation converged (within about 20 days) with that of adsorption alone (Fuller et al., 1993). Thus it is more appropriate to say that coprecipitation is initially more efficient than PSA. In general, higher removal efficiencies can be achieved with increased coagulant or adsorbent dosages for both coprecipitation and PSA (Hering et al., 1996; Cheng et al., 1994; Edwards, 1994; Gulledge \& Oconnor, 1973).

Arsenate and arsenite adsorption have been found to obey a Langmuir isotherm on iron hydroxide and aluminum hydroxide for initial arsenic concentrations in the range $10^{-5}-10^{-3} \mathrm{M}$ at pH 6.0-7.5 (Anderson et al., 1976; Dixit \& Hering, 2003; Ferguson \& Anderson, 1974). Gupta \& Chen (1978) reported similar results for adsorption on alumina and bauxite for initial $\mathrm{pH}$ 6.5-8.5 and initial arsenic concentrations of $5-130 \mu \mathrm{M}$. However, Holm et al. (1979) found adsorption of arsenite on river sediments was linearly dependent on concentration while arsenate adsorption followed a Langmuir isotherm. Initial arsenic levels were $10^{-5}-10^{-4} \mathrm{M}$ and $\mathrm{pH}$ was not mentioned. Pierce \& Moore (1982) found similar linear behavior for high initial concentrations of 33.4-667 $\mu \mathrm{M}$ for both arsenate and arsenite adsorption on amorphous- $\mathrm{Fe}(\mathrm{OH})_{3}$, though a Langmuir isotherm was obeyed at concentrations $0.667-13.3 \mu \mathrm{M}$ in every case. All agree that Langmuir is obeyed for arsenic concentrations found in Bangladesh, where $98 \%$ of wells contain $\mathrm{As}_{\text {tot }}<8$ $\mu \mathrm{M}(\mathrm{BGS}, 2001)$.

The typical adsorption density for $\mathrm{As}[\mathrm{V}]$ on pre-formed hydrous ferric oxide ranges from 0.075 to $0.16 \mathrm{mg} \mathrm{As}[\mathrm{V}]$ per $\mathrm{mg}$ Fe in the $\mathrm{pH}$ range 4-7 (Driehaus et al., 1998; 
Wilkie \& Hering, 1996). For As[III], the adsorption density varies from 0.06 to $0.07 \mathrm{mg}$ As[III] per mg Fe at pH 7.0 (Pierce \& Moore, 1982; Wilkie \& Hering, 1996). Roberts et al. (2004) studied coprecipitation in synthetic Bangladesh groundwater through chemical addition of $\mathrm{Fe}[\mathrm{II}]$ or $\mathrm{Fe}[\mathrm{III}]$ as $\mathrm{FeSO}_{4}$ and $\mathrm{FeCl}_{3}$. For groundwater free of $\mathrm{P}$ and $\mathrm{Si}, \mathrm{As}[\mathrm{V}]$ removal was similar using either $\mathrm{Fe}[\mathrm{II}]$ or $\mathrm{Fe}[\mathrm{III}]$; the addition of $2-2.5 \mathrm{mg} \mathrm{Fe} / \mathrm{L}$ was sufficient to reduce 500 ppb to less than 50 ppb. Less than 1/10 of either Fe[II] or Fe[III] was required to remove As[V] compared to As[III], in agreement with Meng et al. (2002). Borho \& Wilderer (1996) found similar arsenic removal for Fe[II] and Fe[III] in waters low in As, P, and Si. Mamtaz \& Bache (2001) were able to reduce arsenic in synthetic groundwater $\left(1.2 \mathrm{mM} \mathrm{NaHCO}_{3}\right)$ with no P, Si, Ca and $\mathrm{Mg}$ from $200 \mathrm{ppb} \mathrm{As}[\mathrm{III}]$ by $47 \%$ and $43 \%$ using $4 \mathrm{mg} / \mathrm{L} \mathrm{Fe}[\mathrm{II}]$ and Fe[III] respectively after 1 day of settling.

The strength of arsenic adsorption onto iron (hydr)oxides has been widely studied using several experimental techniques, mostly pointing towards formation of an innersphere complex. Some studies focus on the effects of Ionic Strength (I) and pH on arsenic adsorption. The ionic strength quantifies the composite effects of all charged ions in solution, which can impact certain chemical reactions. Changes in I affect the electrostatic forces near the mineral surface (Davis \& Kent, 1990), and thus affect adsorption via outer-sphere complexes much more strongly than inner-sphere complexes. Hsia et al. (1994) and Grossl \& Sparks (1995) show essentially no change in the concentrations of $\mathrm{As}[\mathrm{V}]$ adsorbed by ferrihydrite and goethite for $\mathrm{I}$ in the range of 0.005 to $0.1 \mathrm{M}$, consistent with an inner-sphere complex. Studies of changes in the isoelectric point (IEP) of oxides (the solution $\mathrm{pH}$ at which the net charge on the particle is zero) also indicates 
an inner-sphere complex for both $\mathrm{As}[\mathrm{III}]$ and $\mathrm{As}[\mathrm{V}]$ on goethite, ferrihydrite, gibbsite, and amorphous $\mathrm{Fe}(\mathrm{OH})_{3}$ (Anderson \& Malotky, 1979; Ghosh \& Teoh, 1985; Hingston et al., 1971; Jain et al., 1999; Manning \& Goldberg, 1996a). Finally, Extended X-ray Absorption Fine Structure (EXAFS; discussed more fully in Chapter 4) has confirmed the formation of binuclear bidentate inner-sphere complexes for As $[\mathrm{V}]$ adsorbed onto ferrihydrite (Waychunas et al., 1993; Manceau, 1995) and goethite (Fendorf et al., 1997). Manning et al. (1998) used EXAFS to show similar inner-sphere bonding of As[III] on goethite, confirming results using Fourier Transform IR Spectroscopy (FTIR) by Sun \& Doner (1996). There is some evidence that, in addition to inner-sphere complexes, As[III] may also form outer-sphere complexes on iron oxides (Goldberg \& Johnston, 2001).

Surface precipitation at high concentrations has been suggested as a mechanism for arsenate and phosphate sorption onto ferrihydrite due to slow sorption and high sorptive capacity (Dzombak \& Morel, 1990; van Riemsdijk et al., 1984). However, EXAFS analysis by Waychunas et al. (1993) revealed no such phases in coprecipitation or post-synthesis adsorption of $\mathrm{As}[\mathrm{V}]$ onto Ferrihydrite at $\mathrm{pH} 8.0$ and an ionic strength of $0.02 \mathrm{M}$ during the first seven weeks of aging.

\subsubsection{Arsenic removal via zero valent iron}

The mechanism of arsenic removal in oxic conditions (DO $>0.1 \mathrm{mg} / \mathrm{L})$ via zerovalent iron $(\mathrm{ZVI})$ is adsorption of $\mathrm{As}[\mathrm{V}]$ and $\mathrm{As}[\mathrm{III}]$ oxyanions to ferric oxide precipitates resulting from corrosion of $\mathrm{Fe}(0)$ and subsequent oxidation of $\mathrm{Fe}^{2+}$ (Bang et al., 2005b,a). Mono- and bi-dentate complex formation of arsenic with iron oxides produced from iron 
corrosion has been reported by Lackovic et al. (2000), Farrell et al. (2001) and Su \& Puls (2001). The reactions can be described by the following:

$$
\begin{gathered}
2 \mathrm{Fe}^{0}+\mathrm{O}_{2}+4 \mathrm{H}^{+} \rightleftharpoons 2 \mathrm{Fe}^{2+}+2 \mathrm{H}_{2} \mathrm{O} \\
4 \mathrm{Fe}^{2+}+4 \mathrm{H}^{+}+\mathrm{O}_{2} \rightleftharpoons 4 \mathrm{Fe}^{3+}+2 \mathrm{H}_{2} \mathrm{O} \\
\mathrm{Fe}^{3+}+3 \mathrm{H}_{2} \mathrm{O} \rightleftharpoons \mathrm{Fe}(\mathrm{OH})_{3}+3 \mathrm{H}^{+}
\end{gathered}
$$

The rate of ferric hydroxide addition to the solution is directly related to the corrosion rate. Higher dosages of ferric hydroxide lead to more arsenic removal (Bang et al., 2005b; Mishra \& Farrell, 2005). The corrosion rate is strongly dependent on DO concentration (Equation 2.5) as well as $\mathrm{pH}$, with the required contact time for removal increasing significantly as DO is decreased or pH is increased (Bang et al., 2005b; Lackovic et al., 2000). Both effects are strong - after 9 hours of oxic reaction with $1 \mathrm{~g} / \mathrm{L} \mathrm{Fe}(0)$ filings, 99.8\% removal was achieved for initial As[V] concentrations of $100 \mu \mathrm{g} / \mathrm{L}$ at $\mathrm{pH} 6$, while only $55.5 \%$ and $2 \%$ were removed at $\mathrm{pH} 7$ and 8 respectively (Bang et al., 2005b). In the same time, $99.8 \%$ of $\mathrm{As}[\mathrm{V}]$ was removed at $\mathrm{pH} 6$ in solution open to air compared to only $9 \%$ in $\mathrm{pH} 6$ anoxic solution. The corrosion rate (measured by the total iron content in solution) increases rapidly for about 10 minutes before approaching steady state. In a $0.04 \mathrm{M} \mathrm{NaCl}$ solution under atmospheric conditions at pH 7, Bang et al. (2005a) reported a steady state corrosion rate of about $0.0875 \mathrm{mg} \mathrm{Fe} / \mathrm{min} / \mathrm{L}$. It is important to note that the corrosion rate is very sensitive to surface area.

In the limit of a low ratio of adsorbing species to adsorption sites, the arsenic removal rate is limited by mass transfer to adsorption sites, and is therefore exhibits first 
order dependance on arsenic concentration (Farrell et al., 2001; Melitas et al., 2002b). However, at high solution concentrations, removal becomes limited by the rate of adsorption site generation (related to the corrosion rate and ferric hydroxide formation rate) and is therefore zero order in arsenic concentration (Farrell et al., 2001; Melitas et al., 2002b).

On a weight basis, ZVI is approximately as efficient as coprecipitation with $\mathrm{FeCl}_{3}$. Over 200 days of operation, Bang et al. (2005b) were able to remove 18,000 mg of arsenic using 400g Fe(0) filings - a capacity of $0.045 \mathrm{gAs} / \mathrm{g}$ Fe (including a 1:3 ratio of As[III] to As $[\mathrm{V}])$. However, breakthrough of arsenic began to occur after only 100 days, indicating that filings would have to be replaced long before the capacity was reached.

$\mathrm{As}[\mathrm{III}]$ removal is generally less than $\mathrm{As}[\mathrm{V}]$ removal due to a lower adsorption affinity of $\mathrm{Fe}(\mathrm{OH})_{3}$ for $\mathrm{As}[\mathrm{III}]$ at $\mathrm{pH}<8$ (Bang et al., 2005b; Meng et al., 2000). However, recent research by Hug \& Leupin (2003) shows that As[III] can be oxidized in parallel to the oxidation of Fe[II]. This was attributed to intermediates formed during the reduction of $\mathrm{O}_{2}$ with $\mathrm{Fe}[\mathrm{II}]$. The addition of $90 \mu \mathrm{M} \mathrm{Fe}[\mathrm{II}]$ to $6.6 \mu \mathrm{M}$ As[III] air-saturated solution resulted in $25-30 \%$ oxidation of the As[III] at neutral $\mathrm{pH}$.

\subsubsection{Effect of $\mathrm{pH}$ and speciation on arsenic sorption}

As discussed in Section 2.4, arsenic adsorbs to iron (hydr)oxides by ligand exchange with $\mathrm{OH}$ and $\mathrm{OH}_{2}^{+}$surface functional groups, forming an inner-sphere complex (Stollenwerk, 2003). This requires an incompletely dissociated acid (i.e. $\mathrm{H}_{2} \mathrm{AsO}_{4}^{-}$) to provide a proton for complexation with the surface $\mathrm{OH}$ group, forming $\mathrm{H}_{2} \mathrm{O}$ and providing 
a space for the anion (Hingston et al., 1971). The energy required to dissociate the weak acid at the oxide surface varies with $\mathrm{pH}$, and thus the amount of arsenic adsorbed varies with $\mathrm{pH}$.

The effect of $\mathrm{pH}$ on arsenic adsorption differs between As[III] and As[V], reflecting the difference in net charge of the thermodynamically stable species with $\mathrm{pH}$. Typically, metal oxyanions shift from being mostly adsorbed at low $\mathrm{pH}$ to mostly dissolved at high $\mathrm{pH}$, as $\mathrm{pH}$ increases through a critical range 1-2 $\mathrm{pH}$ units wide (Benjamin, 1983). Although the location of the critical $\mathrm{pH}$ range can be adjusted by changing the system composition, the general shape of the $\mathrm{pH}$-adsorption edge is similar in almost all systems (Benjamin, 1983). Arsenate behaves like metal oxyanions, its adsorption tends to be greatest at low $\mathrm{pH}$ and decreases with increasing $\mathrm{pH}$ (Pierce \& Moore, 1982), attributable to the attraction between a positively charged surface at low $\mathrm{pH}$ and the negatively charged predominant arsenate species $\left(\mathrm{H}_{2} \mathrm{AsO}_{4}^{-}\right)$between $\mathrm{pH} 2.2$ and 6.9 (Stollenwerk, 2003). At pH values above 6.9, the predominant species becomes more negative $\left(\mathrm{HAsO}_{4}^{2-}\right)$, but the surface becomes less positive, making adsorption less favorable. The $\mathrm{pH}$ dependence of $\mathrm{As}[\mathrm{III}]$ is different. The predominant $\mathrm{As}[\mathrm{III}]$ sprecies at $\mathrm{pH}$ less than 9.2 is the neutral $\mathrm{H}_{3} \mathrm{AsO}_{3}^{0}$, more readily able to donate protons to the surface OH group than the negatively charged $\mathrm{As}[\mathrm{V}]$ species.

The relative binding strength of As[III] compared to As[V] depends on the type of iron oxide, the solution $\mathrm{pH}$, and the solution concentration of each species (Pierce \& Moore, 1982; Raven et al., 1998; Manning et al., 1998; Jain et al., 1999). Arsenic adsorption has been observed to be more highly dependent on its oxidation state than 
$\mathrm{pH}$ within the range of 5.5-7.5 (Ferguson \& Anderson, 1974). As[V] removal during coprecipitation with alum, ferric chloride $\left(\mathrm{Fe}^{3+}\right)$, and ferrous sulfate $\left(\mathrm{Fe}^{2+}\right)$ is more efficient than As[III] removal under comparable conditions (Hering et al., 1996; Gulledge \& Oconnor, 1973; Sorg \& Logsdon, 1978; Roberts et al., 2004; Meng et al., 2002; Shen, 1973; Leckie et al., 1980; Meng et al., 2000). However, for PSA experiments on goethite and ferrihydrite (amorphous-HFO), a crossover $\mathrm{pH}$ is often observed with As[V] more efficiently adsorbed below the crossover $\mathrm{pH}$ and As[III] more efficiently adsorbed above it. The value of the crossover $\mathrm{pH}$ and trends with total arsenic concentration vary widely. Dixit \& Hering (2003) report the crossover pH for HFO as 6.5 with 50 or $100 \mu \mathrm{M}$ total arsenic and 8.5 with $10 \mu \mathrm{M}$ total arsenic. Similar observations were made by Raven et al. (1998) for HFO with 534 and $1600 \mu \mathrm{M}$ total arsenic, though at $267 \mu \mathrm{M}$ As[III] was found to be more efficient from $\mathrm{pH} 3-11$, inconsistent with the results of Dixit \& Hering (2003). Pierce \& Moore (1982) report a crossover at pH 6.5 and 7 for total arsenic concentrations of $1.33 \mu \mathrm{M}$ and $13.3 \mu \mathrm{M}$ respectively, reversing the trend seen by Dixit \& Hering (2003). For goethite, the crossover pH was found to be 6.0 with $100 \mu \mathrm{M}, 7.0$ with $50 \mu \mathrm{M}$, and 7.0-8.5 for $25 \mu \mathrm{M}$ by Dixit \& Hering (2003). Manning et al. (1998) reports a crossover $\mathrm{pH}$ for goethite of 5.0 at $267 \mu \mathrm{M}$ and Sun \& Doner (1996) report pH 6.0 at $1250 \mu \mathrm{M}$ total arsenic. These variations may reflect differences in the total sorbate and sorbent concentrations used in the experiments (Dixit \& Hering, 2003). The variation in measurements, including at lower arsenic concentrations relevant to Bangladesh, suggest that it is possible for As[III] adsorption to be comparable or even greater than As[V] adsorption at $\mathrm{pH}$ 7, the typical $\mathrm{pH}$ of Bangladesh groundwater (see Section 3.2.3 below). 
$\mathrm{As}[\mathrm{III}]$ and As$[\mathrm{V}]$ can also compete when in solution together. Jain \& Loeppert (2000) found that As[III] had little effect on adsorption of As[V] below pH 6, but caused a decrease in $\mathrm{As}[\mathrm{V}]$ adsorption by as much as $25 \%$ at $\mathrm{pH}$ greater than 6 due to strong adsorption of As[III]. In contrast, As[V] caused a decrease in adsorption of As[III] by 15-20\% between $\mathrm{pH} 4$ and 6; however by $\mathrm{pH} 9, \mathrm{As}[\mathrm{V}]$ decreased adsorption of As[III] by less than $5 \%$. However, the $\mathrm{pH}$ behavior described above is dominant over competitive adsorption when the concentration of each anion is low relative to the iron precipitate, below $0.1 \mathrm{~mol} \mathrm{As/kg} \mathrm{HFO} \mathrm{(Jain} \mathrm{\&} \mathrm{Loeppert,} \mathrm{2000).}$

\subsubsection{Kinetics of arsenic sorption}

The time dependence of ion sorption by mineral surfaces is often characterized as a two step process (Sposito, 1986; Davis \& Kent, 1990) with initial rapid sorption followed by slower sorption. The slower sorption step can be caused by several different types of processes, including the formation of a surface precipitate (Davis et al., 1987), diffusion to adsorption sites in the internal porosity of minerals (Stollenwerk \& Kipp, 1990), diffusion into the inter-particle porosity of aggregated particles (Wu \& Gschwend, 1988; Willett et al., 1988), or the rate of colloid aggregation and coagulation (Honeyman \& Santschi, 1991). Adsorption onto pre-formed iron (hydr)oxides (PSA) tends to take longer because, as aggregates form, adsorption sites on the surfaces of crystallites become buried within large clusters of particles (Willett et al., 1988). Adsorbing ions react first with the exposed exterior surface sites, but must diffuse within the aggregates before a steady-state concentration of the adsorbing ion can be obtained (Fuller et al., 1993). 
The rate of adsorption tends to be higher during coprecipitation because diffusion into aggregates or clusters is no longer necessary. This is enhanced by the presence of adsorbed ions, which can slow the rates of coagulation and crystallite growth (O'Melia, 1987; Anderson et al., 1985) allowing high rates of adsorption to continue longer. However, as the coprecipitate ages, eventual crystallite growth and increased corner-sharing by Fe octahedra cause desorption of ions (those that cannot be incorporated in a stable three-dimensional structure) (Fuller et al., 1993). This means that some arsenic could be expelled from the crystallizing matrix as it ages, slowing or even reversing the net rate of arsenic adsorption.

The kinetics of adsorption onto amorphous ferrihydrite, and many other oxides, also includes an initial rapid step, typically between 5-30 min, and is sometimes followed by a slower step during which the adsorption density may increase at a rate of a few percent per day (Benjamin, 1983; Kurbatov \& Wood, 1952; Posselt et al., 1968). Fuller et al. (1993) reported an initial rapid uptake of $\mathrm{As}[\mathrm{V}](<5 \mathrm{~min})$ by ferrihydrite in solution at $\mathrm{pH} 8$ followed by a slow uptake (over 8 days) consistent with diffusion to adsorption sites within aggregates or colloidal particles ( $\mathrm{pH}$ was maintained throughout the experiment). (Grossl et al., 1997) used a pressure/jump relaxation technique on goethite to find that the formation of monodentate ferric-arsenate surface complexes form on a time scale of milliseconds while transformation to bidentate complexes occurred on a time scale of seconds. This provides further evidence that equilibration times on the order of hours to days are due to slow diffusion rather than complex formation.

Coprecipitation experiments producing equivalent amounts of ferrihydrite as 
the above showed much higher adsorption rates $(\mathrm{pH} 7.5-9.0)$, with $>95 \% \mathrm{As}[\mathrm{V}]$ adsorbed after 5 min and approximately twice as much As[V] adsorbed after 24 hours compared to PSA experiments. After the initial uptake, the coprecipitate began to release As[V], continuing after 750 hours of reaction, though the final amount adsorbed was still greater than in the PSA case. $\mathrm{Fe}^{2+}$ derived coprecipitates generally had less initial sorption than similar systems with $\mathrm{Fe}^{3+}$ derived coprecipitates, however they tended to desorb more slowly with aging. EXAFS analysis on PSA and coprecipitation samples revealed no surface precipitate or solid-solution formed, pointing to diffusion as the rate limiting step (Waychunas et al., 1993; Fuller et al., 1993).

Numerous researchers have chosen equilibration times for adsorption batch experiments with either no reported explanation or no supporting data. Equilibration times used for As[V] and As[III] on goethite include 24 hours (Grossl et al., 1997; Hingston et al., 1971; Dixit \& Hering, 2003), 16 hours (Manning et al., 1998), 2 hours (Sun \& Doner, 1996), and overnight (Farquhar et al., 2002). Equilibration times used for As[III] and $\mathrm{As}[\mathrm{V}]$ on ferrihydrite and amorphous-iron oxide include 24 hours (Jain et al., 1999; Raven et al., 1998), 4 hours (citing Pierce \& Moore (1982); Dixit \& Hering (2003)), 2 hours (Hsia et al., 1992; Hering et al., 1996; Wilkie \& Hering, 1996) and 20 minutes (Goldberg \& Johnston, 2001). Equilibration for coprecipitation experiments with ferric salts include 4 hours (Roberts et al., 2004), 1 hour (citing internal kinetics tests; Meng et al. (2000)) and a combination of 1 min rapid mixing, 30 min slower mixing, and 15 min quiescent settling (Hering et al., 1996). Dixit \& Hering (2003) used 24 hours equilibration for As[III] adsorption onto magnetite, citing internal kinetics tests (no details 
given).

The kinetics of arsenic removal via ZVI depends on the rates of several subprocesses, including the corrosion rate of $\mathrm{Fe}(0)$ (which depends on the DO concentration and $\mathrm{pH}$ ), the rate of formation of ferric hydroxide precipitates, as well as the rate of arsenic adsorption onto the precipitates. Bang et al. (2005a) reports a steady state of soluble iron formation from $\mathrm{Fe}(0)$ and ferric hydroxide formation after only 10 minutes in solution. Bang et al. (2005b) was able to fit a psuedo first-order reaction obtaining removal half lives for $\mathrm{As}[\mathrm{III}]$ and $\mathrm{As}[\mathrm{V}]$ via $\mathrm{ZVI}$ at $\mathrm{pH} 6$ of 3.96 and 2.14 hrs respectively. At $\mathrm{pH} 7$, the half life for As[V] increased to 8.72 hours (half-life of As[III] could not be measured from the data at pH 7). Su \& Puls (2001) cite half-lives of 9-131 hours for As $[\mathrm{V}]$ removal on a variety of commercially available Fe(0) products. Farrell et al. (2001) and Melitas et al. (2002b) described the arsenate removal kinetics by corroding iron in batch tests using a model of the form:

$$
\frac{d[A s]}{d t}=\frac{-k_{0}[A s]}{k_{0} / k_{1}+[A s]}
$$

where $[\mathrm{As}]$ is the arsenate solution concentration, $t$ is time, $k_{0}$ is the zeroth-order rate constant, and $k_{1}$ is the first-order rate constant. Removal rates are first-order in [As] at the limit of infinite dilution and zeroth-order in [As] at infinite concentration. Firstorder kinetics occur in situations with excess adsorption sites (and hence no sorptive competition), limited by mass-transfer to adsorption sites. Deviations begin as the rate of $\mathrm{Fe}^{2+}$ generation becomes less than the rate of removal, inducing competition between species for a decreasing number of adsorption sites. At a fixed corrosion rate, competition increases with arsenic concentration. At high arsenic concentrations, removal kinetics 
may approach zeroth-order, limited by the rate of adsorption site generation (Melitas et al., 2002b).

It has been reported that arsenate reacts with amorphous iron oxides much faster than arsenite; Pierce \& Moore (1982) found arsenite adsorption onto pre-formed am- $\mathrm{Fe}(\mathrm{OH})_{3}$ to be $90 \%$ complete after 2 hours (final $\mathrm{pH}$ after 24 hours 6.1 and 9.8), while arsenate adsorption was $90 \%$ complete after only 1 hour (final pH after 24 hours 8.0 and 9.9). Adsorption of both anions was $99 \%$ complete after 4 hours (initial As concentration of $0.150 \mathrm{~mol} \mathrm{As} / \mathrm{kg}$ am- $\left.\mathrm{Fe}(\mathrm{OH})_{3}\right)$. However, Raven et al. (1998) found arsenite adsorbed faster to pre-formed ferrihydrite than arsenate at pH 9.2 (maintained throughout reaction), reaching $99.96 \%$ of the uptake at 96 hours after 1 hour compared to $99.69 \%$ for arsenate after 1 hour (initial As concentration of 0.267 mol As/ kg Ferrihydrite). Both Raven et al. (1998) and Pierce \& Moore (1982) found noticeably higher adsorption rates for higher initial arsenic concentrations (13.3 mol As/kg Ferrihydrite and $2.9 \mathrm{~mol} \mathrm{As} / \mathrm{kg}$ am- $\mathrm{Fe}(\mathrm{OH})_{3}$ respectively) which could help to explain the discrepancy between results. Fuller et al. (1993) has challenged the results of Pierce \& Moore (1982) based on their failure to maintain a constant $\mathrm{pH}$ throughout the reaction time. Bang et al. (2005b) found As[III] removal to be slower than As[V] removal at pH 7 using ZVI under an oxic environment, reaching only $55 \%$ for As[III] compared to $72 \%$ for As[V] after 90 minutes of mixing. 


\subsubsection{Effects of co-occuring solutes}

Many inorganic and organic aqueous species can affect arsenic adsorption. They can compete with arsenic directly for available binding sites or indirectly by altering the electrostatic charge at the adsorbent surface. Both processes are influenced by $\mathrm{pH}$, ion concentration, and the intrinsic binding affinity of the adsorbent (Davis \& Kent, 1990). Arsenic affected (As $>10 \mathrm{ppb}$ ) groundwater in Bangladesh is known to contain phosphate, silica, sulfate, bicarbonate, calcium, and magnesium among others (see Table 3.1), each of which may affect arsenic sorption onto iron (hydr)oxides in different ways.

\section{Phosphate}

Spectroscopic studies have shown than phosphate $\left(\mathrm{PO}_{4}^{3-}\right)$ forms identical surface species to arsenate (Waychunas et al., 1993; Hiemstra \& Van Riemsdijk, 1999). The affinity of $\mathrm{P}[\mathrm{V}]$ for surface sites is also similar (Hingston et al., 1971; Manning \& Goldberg, 1996a,b; Ryden et al., 1987; Gao \& Mucci, 2001; Liu et al., 2001). Jain \& Loeppert (2000) studied the influence of $\mathrm{P}[\mathrm{V}]$ on adsorption of $\mathrm{As}[\mathrm{III}]$ and $\mathrm{As}[\mathrm{V}]$ on ferrihydrite as a function of $\mathrm{pH}$ for $\mathrm{P}[\mathrm{V}]:$ Astot ratios of $1: 1$ and 10:1 - within the range reported for many groundwaters, including those in Bangladesh (BGS, 2001). They found that adsorption of both $\mathrm{As}[\mathrm{V}]$ and $\mathrm{As}[\mathrm{III}]$ decreased with increasing $\mathrm{P}[\mathrm{V}]$ concentration, and for $\mathrm{As}[\mathrm{V}]$ this was significant over the entire $\mathrm{pH}$ range. At $\mathrm{pH} 7, \mathrm{As}[\mathrm{V}]$ adsorption decreased from about $96 \%$ to $50 \%$ between no $\mathrm{P}[\mathrm{V}]$ and the highest concentration $\left(\mathrm{P}[\mathrm{V}]: \mathrm{As}_{\text {tot }}=\right.$ 10:1). At the same $\mathrm{pH}, \mathrm{As}[\mathrm{III}]$ adsorption decreased from about $96 \%$ to $74 \%$. At $\mathrm{pH}$ 9, the decrease in As[III] adsorption was only a few percent, even at the highest $\mathrm{P}[\mathrm{V}]$ 
concentration, indicating that the neutral $\mathrm{H}_{3} \mathrm{AsO}_{3}^{0}$ dominant at this $\mathrm{pH}$ is better able to compete with negatively charges phosphate species. Other researchers have found similar effects. Hingston et al. (1971) measured an 85\% decrease in As[V] adsorption by goethite when the $\mathrm{P}[\mathrm{V}] / \mathrm{As}[\mathrm{V}]$ ratio was increased from 0 to 12:1. Manning \& Goldberg (1996a) reported that a $\mathrm{P}[\mathrm{V}] / \mathrm{As}[\mathrm{V}]$ ratio of $1: 1$ caused a $30 \%$ decrease in $\mathrm{As}[\mathrm{V}]$ adsorption by both goethite and gibbsite compared to $\mathrm{P}[\mathrm{V}]$-free solutions at $\mathrm{pH}$ values less than 8. It is important to note that the above studies looked at competitive adsorption on iron (hydr)oxides formed prior to the addition of arsenic and other ions.

The order in which phosphate and arsenic are added to solution is an important factor in determining competitive effects. Liu et al. (2001) found an adsorption density of $78 \mathrm{mmol} \mathrm{As}[\mathrm{V}] / \mathrm{kg}$ goethite when equal amounts of $\mathrm{As}[\mathrm{V}]$ and $\mathrm{P}[\mathrm{V}]$ were added to solution at the same time (at $\mathrm{pH} 7$ ) compared to only $51 \mathrm{mmol} \mathrm{As}[\mathrm{V}] / \mathrm{kg}$ goethite when the $\mathrm{P}[\mathrm{V}]$ was added 24 hours before $\mathrm{As}[\mathrm{V}]$ and $139 \mathrm{mmol} \mathrm{As}[\mathrm{V}] / \mathrm{kg}$ goethite when As[V] was added alone. Some researchers have also reported desorption of arsenic on iron oxides in the presence of phosphate (Alam et al., 2001; Jackson \& Miller, 2000).

The structure and particle size of iron (hydr)oxides can also be affected by phosphate. Mayer \& Jarrell (2000) found that crystallite growth was slower in the presence of phosphate. Lytle \& Snoeyink (2002) found that the particle size of iron oxide suspension, as observed through photon correlation spectroscopy, decreased in the presence of phosphate. In addition, polyphosphates are commonly added to drinking water to prevent iron from precipitating out of solution, though the more stable orthophosphate form is more likely to be present in natural waters. 


\section{Silicate}

The iron (hydr)oxide surface has a strong affinity for silicate (Sigg \& Stumm, 1981), however silicate adsorption is weaker than As[V] adsorption (Swedlund \& Webster, 1999). Silicic acid $\left(\mathrm{H}_{4} \mathrm{SiO}_{4}\right)$ has been shown to effectively compete with arsenic for adsorption sites (Davis et al., 2001). The adsorption of As[V] was found to decrease with increasing pH and silicate concentration (Holm, 2002) and Waltham \& Eick (2002) reported that silicate increased iron mobilization and interfered with arsenic removal. Meng et al. (2000) looked at Si and As adsorption onto ferrihydrite as ferrihydrite precipitated from solution. Adsorption of $\mathrm{As}[\mathrm{III}](300 \mu \mathrm{g} / \mathrm{L})$ and $\mathrm{As}[\mathrm{V}](500 \mu \mathrm{g} / \mathrm{L})$ at pH 6.8 began to decrease in the presence of $1 \mathrm{mg} / \mathrm{L} \mathrm{Si}$; at $10 \mathrm{mg} / \mathrm{L} \mathrm{Si}$, adsorption decreased by $70 \%$ for $\mathrm{As}[\mathrm{V}]$ and $80 \%$ for As[III]. Ferrihydrite that had been aged for 18-24 hours prior to adding $3.1 \mathrm{mg} / \mathrm{L} \mathrm{Si} \mathrm{showed} \mathrm{little} \mathrm{effect} \mathrm{on} \mathrm{adsorption} \mathrm{of} \mathrm{As}[\mathrm{V}]$ and $\mathrm{As}[\mathrm{III}]$ at $\mathrm{pH}<8$ (Swedlund \& Webster, 1999). In the presence of $56 \mathrm{mg} / \mathrm{L} \mathrm{Si}$, As[III] adsorption decreased between $\mathrm{pH} 4$ and 10 by as much as 35\%, and As[V] asorption decreased by as much as $60 \%$ above $\mathrm{pH}$. Swedlund \& Webster (1999) attributed the decrease in arsenic adsorption to surface site competition and polymerization of Si resulting in negative surface charge.

Silicate has also been known to affect the structure of iron (hydr)oxides. Silicate can affect the particle size and crystallinity of iron (hydr)oxides (Rushing et al., 2003; Davis et al., 2001; Meng et al., 2000; Deng, 1997; Anderson \& Benjamin, 1985). Mayer \& Jarrell (2000) found that the presence of Si during Fe[II] oxidation at molar ratios above $0.36 \mathrm{Si} /$ Fe promoted the formation of ferrihydrite, though it inhibited coagulation 
and crystallite growth. Anderson \& Benjamin (1985) similarly found the presence of silicate delayed ferrihydrite crystallization into goethite from $24 \mathrm{hr}$ to $1-2$ weeks. Davis et al. (2001) found that silicate sorption to iron oxides produced a highly negative surface charge, leading to the creation of smaller iron particles from larger particles. The effects of Si on iron (hydr)oxides mineralogy may be more important than the competition between $\mathrm{Si}$ and As for sorption sites. Increased sorption capacities of Fe oxides for phosphate (similar in structure to arsenate) in the presence of silica has been attributed to the effect of $\mathrm{Si}$ on Fe oxide mineralogy as opposed to competition between $\mathrm{P}$ and $\mathrm{Si}$ for sorption sites (Mayer \& Jarrell, 2000). However, the increased sorption capacity for phosphate could also increase the competitive edge of phosphate compared to arsenic in sorption site competition, enhancing the detrimental effect of phosphate.

There has been some evidence that silica can catalyze the oxidation of Fe[II] to $\mathrm{Fe}[\mathrm{III}]$ in aerated water in the pH range of 6.6 to 7.1 (Schenk \& Weber, 1968). However, Rushing et al. (2003) found that silica significantly slowed Fe[II] oxidation to Fe[III] in aerated water at $\mathrm{pH} 6.5$.

\section{Carbonate}

Carbonate species exist in natural waters as $\mathrm{H}_{2} \mathrm{CO}_{3}^{0}, \mathrm{HCO}_{3}^{-}$, and $\mathrm{CO}_{3}^{2-}$. There is some evidence of carbonate species adsorption onto iron (hydr)oxides (Villalobos \& Leckie, 2000; van Green et al., 1994; Zachara et al., 1987). It is usually described as an outer-sphere complex, but spectroscopic evidence suggests the formation of a monodentate inner-sphere complex (Villalobos \& Leckie, 2000; van Green et al., 1994). Meng 
et al. (2000) found essentially no effect of carbonate on adsorption of As[III] and As[V] on ferrihydrite in equilibrium with air and with a total carbonate concentration of $0.01 \mathrm{mM}$. Little effect was found on adsorption of $\mathrm{As}[\mathrm{V}]$ during coprecipitation in stream water with 3mM carbonate at pH 8 (Fuller et al., 1993). There is some evidence that carbonate may affect adsorption of As[III] but not As[V]. Wilkie \& Hering (1996) found that $1 \mathrm{mM}$ carbonate caused about a $10 \%$ decrease in $\mathrm{As}[\mathrm{III}]$ adsorption on ferrihydrite at $\mathrm{pH}$ 9 and only a few percent decrease in $\mathrm{As}[\mathrm{V}]$; no effect was seen at $\mathrm{pH}$. Some evidence also exists that adsorption of carbonate by ferrihydrite in a shallow aquifer ( $5 \mathrm{mM}$ carbonate, $7.1>\mathrm{pH}>7.7)$ may have decreased adsorption of As[III], and to a lesser extent As[V] (Appelo et al., 2002). High arsenic concentrations in groundwater have also been correlated with high bicarbonate concentrations (Nickson et al., 2000; Kim et al., 2000).

\section{Calcium and Magnesium}

Some solutes can reportedly enhance adsorption of arsenic. Wilkie \& Hering (1996) found an increase in As[V] adsorption by ferrihydrite when calcium was also adsorbed at $\mathrm{pH} 9$, apparently by increasing the positive charge on the surface. Meng et al. (2000) also noted that additions of calcium and magnesium to ferrihydrite suspensions negated part of the competitive effect of silica on arsenic adsorption. The presence of calcium has also enhanced adsorption of $\mathrm{As}[\mathrm{V}]$ on aluminum oxides at $\mathrm{pH}>8$ (Ghosh \& Teoh, 1985). 


\section{Sulfate}

Macroscopic chemical evidence suggests that sulfate $[\mathrm{S}(\mathrm{VI})]$ adsorbs via an outer-sphere complex and should therefore have much less effect on arsenic adsorption than inner-sphere complex formers such as phosphate (Stollenwerk, 2003). Jain \& Loeppert (2000) studied the influence of sulfate at S/As ratios of 10:1 and 50:1 on As[III] and $\mathrm{As}[\mathrm{V}]$ adsorption to ferrihydrite from $\mathrm{pH} 3-11$. They found essentially no effect on As[V] adsorption over the entire range, though an increase of a few percent was observed for the highest S/As ratio, attributed to an ionic strength effect. There was a slight decrease in As[III] adsorption at $\mathrm{pH}<7$, but no effect at higher $\mathrm{pH}$. Similar results were observed by Wilkie \& Hering (1996). As[III] adsorption by ferrihydrite decreased $20 \%$ at $\mathrm{pH} 7$ and $70 \%$ at $\mathrm{pH} 4$ in the presence of 4000:1 S/As, while As[V] adsorption decreased by only a few percent at $\mathrm{pH} 5$.

\section{Combined effect of ions}

Meng et al. (2002) looked at the combined effect of phosphate, silicate, and bicarbonate on the removal of arsenic from Bangladesh groundwater and simulated groundwater by iron (hydr)oxides. They found that phosphate $(0-0.08 \mathrm{mM})$, silicate $(0-0.8 \mathrm{mM})$, and bicarbonate $(0-14 \mathrm{mM})$ in separate solutions had none to moderate effects on $\mathrm{As}[\mathrm{V}]$

removal in solutions with $6.7 \mathrm{mg} / \mathrm{L} \mathrm{Fe}$ and $300 \mathrm{ppb} \mathrm{As}[\mathrm{V}]$. However, the presence of bicarbonate and silicate was found to magnify the adverse effect of phosphate such that the residual As[V] concentration increased 10 fold. Roberts et al. (2004) investigated the amount of $\mathrm{Fe}[\mathrm{II}]$ and $\mathrm{Fe}[\mathrm{III}]$ required in coprecipitation experiments to reduce $500 \mathrm{ppb}$ 
of $\mathrm{As}[\mathrm{III}]$ and $\mathrm{As}[\mathrm{V}]$ to below $50 \mathrm{ppb}$ with and without $30 \mathrm{mg} / \mathrm{L} \mathrm{Si}$ and $3 \mathrm{mg} / \mathrm{L} \mathrm{P.} \mathrm{Over}$ 2.5 and 7 times the Fe[III] was needed for $\mathrm{As}[\mathrm{III}]$ and $\mathrm{As}[\mathrm{V}]$ respectively in the presence of Si and P. Using Fe[II] additions, about 1.5 and 5 times as much was needed for As[III] and As[V] respectively. Mayer \& Jarrell (2000) found that the presence of silica during $\mathrm{Fe}[\mathrm{II}]$ oxidation greatly increased the sorption density and retention capacity of the resulting ferrihydrite for phosphate. This could potentially increase the detrimental effect of phosphate on arsenic adsorption if the affinity of iron (hydr)oxides for phosphate is increased more than the affinity of iron (hydr)oxides for arsenate or arsenite.

The presence of co-occuring solutes can also affect the relative efficiency of arsenic removal via coprecipitation with $\mathrm{Fe}^{2+}$ and $\mathrm{Fe}^{3+}$ salts. Roberts et al. (2004) observed that for synthetic groundwater free of $\mathrm{P}$ and $\mathrm{Si}, \mathrm{As}[\mathrm{V}]$ removal was similar using either $\mathrm{Fe}^{2+}$ or $\mathrm{Fe}^{3+}$, however in the presence of $30 \mathrm{mg} / \mathrm{L} \mathrm{Si}$ and $3 \mathrm{mg} / \mathrm{L} \mathrm{P}$, both $\mathrm{As}[\mathrm{V}]$ and $\mathrm{As}[\mathrm{III}]$ were more efficiently removed by the addition of $\mathrm{Fe}^{2+}$, with the effect being more pronounced for As[III].

\subsection{Electrocoagulation}

\subsubsection{Electrochemical cell theory}

To help follow the rest of the thesis with minimal reference to external texts, below is a summary of published material heavily based on Bard \& Faulkner (2001), but also embellished with additional references, inferences, and recorded observations.

A simple electrochemical cell consists of two electrical conductors (electrodes) 
separated by an ionic conductor (electrolyte). Charge is transported in the electrodes by the movement of electrons (and holes). Charge is transported in the electrolyte by the movement of ions. The boundary between two chemical phases, such as the solid electrode and aqueous electrolyte, is called the interface, and numerous processes and factors can affect the transport of charge across the interface. The difference in electric potential between the electrodes is called the cell potential, measured in volts (V), and is a measure of the energy available to move charge between electrodes. It is the sum of the electric potential across all of the various phases in the cell. The transition in electric potential crossing from one conducting phase to another (e.g. electrode to electrolyte) occurs almost entirely at the interface (Bard \& Faulkner, 2001), implying that high electric fields can exist in this region. The magnitude of the potential difference at the interface affects the energies of the charge carriers on either side of the interface, and hence it controls the direction and rate of charge transfer. The potential difference at each electrode/electrolyte interface controls the half-reaction occurring there, and hence the real chemical changes at the electrode (in turn controlling the chemical composition near the electrode). Commonly, one is interested in reactions occurring at only one of the electrodes, and that electrode is called the working or indicator electrode.

The cell potential is the sum of many potential differences across the cell, including each interface between phases and the ohmic drop across the electrolyte. To measure or control the potential at the working electrode alone, the non-working electrode is often a reference electrode, made up of components with essentially constant composition capable of maintaining a constant interface potential over a wide range of currents. The 
ohmic drop can be minimized by reducing the bulk solution resistance or using a three electrode cell (discussed in more detail below). The internationally accepted primary reference is the standard hydrogen electrode ( $\mathrm{SHE}$ ) in which $\mathrm{H}_{2}$ gas is bubbled near a platinum wire and all components have a unit activity. Standard potentials of a half reaction are measured in a simple electrochemical cell in which one electrode is a SHE. Other common reference electrodes include the saturated calomel electrode (SCE) with a potential of 0.242 V vs. SHE (Bard \& Faulkner, 2001) and the silver-silver chloride electrode with a potential of 0.197 V vs. SHE (Bard \& Faulkner, 2001). Control over the potential of the working electrode is thus achieved only with respect to the constant potential of the reference.

Driving the potential of the working electrode to more negative values (e.g. by connecting a battery or power supply to the cell with its negative side attached to the working electrode) will raise the energy of the electrons in the electrode. They can reach an energy high enough to transfer into vacant states on a species in the electrolyte, creating a flow of electrons from electrode to solution known as a reduction current. Similarly, driving the potential of the working electrode to more positive values can lower the energy of the electrons in the electrode enough for electrons on solutes in the electrolyte to transfer to more favorable energy states on the electrode, creating a flow from solution to electrode known as an oxidation current. The critical potentials at which these processes occur are related to the standard potentials, $E^{0}$, for the specific chemical substances in the system. The standard potential is measured in a cell where all the constituents are in their standard states (i.e. $1 \mathrm{M}$ at $25^{\circ} \mathrm{C}$ and 1 bar total pressure). 
Frequently, the concentrations of the reduced or oxidized species are different from 1M. Consider the reaction:

$$
\mathrm{O}+\mathrm{n}_{\mathrm{e}} \mathrm{e}^{-} \rightleftharpoons \mathrm{R}
$$

$O=$ Oxidized species

$R=$ Reduced species

In this case the critical potential is given by the Nernst equation:

$$
E=E^{0^{\prime}}+\frac{R T}{n_{e} F} \ln \frac{\left[C_{O}\right]}{\left[C_{R}\right]}
$$

$R=$ Universal gas constant $[\mathrm{kJ} / \mathrm{mol}-\mathrm{K}]$

$T=$ Temperature $[\mathrm{K}]$

$n_{e}=$ number of electrons in redox reaction

$F=$ Faraday's constant $\left[96,485.4 \mathrm{C} / \mathrm{mol} e^{-}\right]$

$C_{O}=$ Concentration of oxidized species $[\mathrm{M}]$

$C_{R}=$ Concentration of reduced species $[\mathrm{M}]$.

Here, $E^{0^{\prime}}$ is the formal potential, related to $E^{0}$ by:

$$
E^{0^{\prime}}=E^{0}+\frac{R T}{n_{e} F} \ln \frac{\gamma_{O}}{\gamma_{R}}
$$

$\gamma_{O}=$ activity coefficient of oxidized species

$\gamma_{R}=$ activity coefficient of reduced species.

The take-away from Equations 2.10 and 2.11 is that the critical potential depends on environmental conditions, such as temperature, as well as the relative concentrations of the reactants near the electrode surface and the activity of those reactants.

For faradic processes, the number of electrons that cross an interface is related stoichiometrically to the extent of chemical reaction (i.e. the amount of reactant consumed and product generated). Current is the total charge passed per unit time, and 
thus the current is a measure of the rate of chemical reactions occurring in the cell. Under some conditions, a given electrode/solution interface will show a range of potentials where no charge-transfer reactions occur because they are thermodynamically or kinetically unfavorable. However, adsorption or desorption reactions can occur that change the structure of the electrode-solution interface without changing the potential or solution composition. These are called non-faradic processes and can sometimes lead to transient currents as the electrode area, potential, or solution composition changes. These currents are not associated with charge-transfer. When one plots the current as a function of potential, one obtains a current-potential ( $i$ vs. E) curve including information about both faradic and non-faradic processes. Such curves can be very informative about the reactions occurring at the electrode (this is the basis of the transient voltammetry technique used in Chapter 3).

If well-defined redox couples exist at each electrode, then equilibrium can be established and the cell will have a well defined equilibrium potential, or open circuit potential, $E_{e q}$. This is the potential one would measure across the electrodes if no net current was flowing. In many cases, there is no well-defined equilibrium state for the cell, and the open circuit potential can only be placed within a potential range. The departure of the electrode potential from the equilibrium value upon the passage of a faradic current is termed polarization. The extent of polarization is measured by the overpotential, $\eta$ :

$$
\eta=E-E_{e q}
$$

The overpotential $\eta$ can be considered a sum of terms associated with different reaction 
steps:

$$
\eta=\eta_{M t}+\eta_{C t}+\eta_{r x n}
$$

$\eta_{M t}=$ mass-transfer overpotential [Volts]

$\eta_{C t}=$ charge-transfer overpotential [Volts]

$\eta_{\text {rxn }}=$ the overpotential associated with a preceding reaction [Volts]

$\eta_{M t}$ is the overpotential necessary to drive mass-transfer, the physical movement of ions from the bulk solution to the electrode surface where reactions take place (also called the concentration overpotential or diffusion overpotential). It is caused by the change in solute concentrations occurring in the proximity of the electrode surface due to the electrode reaction. The mass-transfer overpotential can be reduced by increasing the transport of ions from anode surface to the bulk solution, i.e. by increased mixing between electrodes. $\eta_{M t}$ becomes negligible when the reaction rate constant is much smaller than the mass-transfer coefficient (Mollah et al., 2004). The charge-transfer overpotential, $\eta_{C t}$, is the energy associated with electron transfer at the electrode surface and $\eta_{r x n}$ is energy associated with the chemical reactions that precede or follow electron transfer. The sum of $\eta_{C t}$ and $\eta_{r x n}$ is sometimes called the kinetic overpotential, $\eta_{K}$.

Both $\eta_{K}$ and $\eta_{M t}$ increase as the current density, $j=i / A$, increases. A precise relationship can be derived in the simple case of a one-step, one-electron process, leading to the Butler-Volmer formulation. For the case of interface equilibrium and a solution in which the bulk oxidized species concentration is equal to the bulk reduced species concentration, the Butler-Volmer formulation is (see Bard \& Faulkner (2001), section 3.3.3 for a detailed derivation):

$$
j=F k^{0}\left[C_{O}(0, t) e^{-\alpha f \eta}-C_{R}(0, t) e^{(1-\alpha) f \eta}\right]
$$


$j=$ Current Density

$F=$ Faraday's constant

$k^{0}=$ standard rate constant for the reaction

$C_{O}(0, t)=$ concentration of oxidized species at the electrode surface as a function of time

$C_{R}(0, t)=$ concentration of reduced species at the electrode surface as a function of time

$\alpha=$ transfer coefficient, ranging from zero to unity

$f=\mathrm{F} / \mathrm{RT}$

$\eta=$ overpotential at the electrode

The standard rate constant, $k^{0}\left(s^{-1}\right)$, is the rate constant for the reaction when $\eta=0$. The transfer coefficient $\alpha$ is a property of the system. Equation 2.14 demonstrates the tendency for the current density to be related exponentially to the overpotential $\eta$. This behavior is seen in many electrochemical systems, both simple and complicated, as first noted by Tafel in 1905 .

The applied cell potential necessary to get the desired current must take into account the potential across the working electrode, $E$ (which includes the overpotential), as well as the voltage drop across the solution due to the bulk solution resistance, $R_{s}$. Using the convention of positive current, $i$, for oxidation (or anodic) current ${ }^{1}$, the applied cell potential, $E_{a p p l}$ is:

$$
E_{a p p l}=E_{e q}+\eta+i R_{s}
$$

The solution resistance, $R_{s}(\Omega)$, is determined by:

$$
R_{s}=\frac{d}{A \cdot \kappa}
$$

\footnotetext{
${ }^{1}$ Note that this is opposite the convention used in Bard \& Faulkner (2001)
} 
$d=$ distance between electrodes $[\mathrm{m}]$

$A=$ active surface area of anode $\left[\mathrm{m}^{2}\right]$

$\kappa=$ specific conductivity of bulk solution $\left[10^{3} \mathrm{mS} / \mathrm{m}\right]$

The bulk resistance $R_{s}$, and hence the $i R_{s}$-drop, can be reduced by decreasing the distance between electrodes, increasing the submerged surface area of anode, or increasing the specific conductivity of the bulk solution.

For precise measurements of the working electrode interface potential in low conductivity solution, a three-electrode cell is often used. In this configuration, current is passed between the working electrode and a counter electrode. The counter electrode can be any convenient metal, because its electrochemical properties do not affect the behavior of the electrode of interest, though care must be taken that it will not produce solutes that can reach the working electrode and cause interfering reactions. A reference electrode is added with its tip very close to the working electrode to minimize the $i R_{s^{-}}$ drop. The working electrode potential is measured using a high impedance voltmeter between the working electrode and the reference electrode, allowing almost all of the current to pass through the cell from working to counter electrode, and negligibly small current to pass between the working and reference electrode. Even in this case, if the reference electrode is not touching the working electrode (it must be at least $2 d$ away from the working electrode to avoid shielding errors if its tip diameter is $d$ ), some fraction of $i R_{s}$, known as the uncompensated resistance, will still be measured on the voltmeter. 


\subsubsection{Electrocoagulation (EC) using iron}

Electrocoagulation (EC) is a complicated process in which consumable electrodes supply ions that can be used in situ as coagulants or sorbates. When the electrodes of an electrochemical cell are connected to an electrical DC power source, the pull of electrons from the anode will oxidize the anode metal, causing metal cations to dissolve into solution. In the case of an iron anode, the oxidized metal ions quickly hydrolyze to form insoluble polymeric iron (hydr)oxides, providing an active surface for sorption of contaminants, as well as excellent coagulating agents. Coagulation occurs when the metal cations combine with negative particles carried towards the anode by electrophoretic motion (Mollah et al., 2004). The negative ions neutralize ionic iron species in the solution, reducing electrostatic interparticle repulsion until van der Waals attraction predominates, aiding coagulation and aggregation into flocs (Mollah et al., 2004). The flocs create a sludge blanket able to entrap and bridge colloidial particles (Mollah et al., 2004), removing contaminants while enhancing further aggregation. Contaminants can precipitate directly or physically and chemically attach to the aggregating iron (hydr)oxides. This allows for the removal of colloidal contaminants via filtration or sedimentation of the aggregated iron (hydr)oxides they are attached to. In addition to the processes described above, some of the following may also occur in the electrocoagulation cell, which may hinder or aid contaminant removal (Mollah et al., 2004):

- Cathodic reduction of impurities.

- Anodic oxidation of impurities.

- Discharge and coagulation of colloidal contaminant particles. 
- Electrophoretic migration of ions in solution, increasing the probability of collisions (Persin \& Rumeau, 1989).

- Electroflotation of coagulated particles by $\mathrm{O}_{2}$ and $\mathrm{H}_{2}$ bubbles produced at the electrodes (bubbles can float contaminant flocs to the surface).

- Reduction of metal ions at the cathode.

- Other electrochemical and chemical processes.

The production of iron (hydr)oxides from the electrochemical dissolution of an iron anode may proceed through a number of different chemical reactions. Oxidation at the anode can produce $\mathrm{Fe}^{2+}$ or $\mathrm{Fe}^{3+}$ according to either:

$$
\mathrm{Fe}_{(\mathrm{s})} \rightleftharpoons \mathrm{Fe}_{(\mathrm{aq})}^{2+}+2 \mathrm{e}^{-}
$$

or

$$
\mathrm{Fe}_{(\mathrm{s})} \rightleftharpoons \mathrm{Fe}_{(\mathrm{aq})}^{3+}+3 \mathrm{e}^{-} \text {. }
$$

For Faradic processes, the theoretical amount of $\mathrm{Fe}^{3+}$ or $\mathrm{Fe}^{2+}$ ions, $w_{t h}(\mathrm{mg})$, dissolved into solution at current $i$ in current processing time $t_{C P}$ is given by Faraday's law:

$$
w_{t h}=\frac{i \cdot t_{C P} \cdot M}{n F}
$$

$i=$ operating current $(\mathrm{A})$

$t_{C P}=$ current processing time $(\mathrm{s})$

$M=$ molecular weight (for iron, $\mathrm{M}=55.845 \mathrm{~g} / \mathrm{mol}$ )

$n=$ number of electrons involved in the oxidation/reduction reaction ( $\mathrm{n}=2$ or 3 for $\mathrm{Fe}^{2+}$ or $\mathrm{Fe}^{3+}$ respectively)

$F=$ Faraday's constant. $96,500 \mathrm{C} / \mathrm{mol} \mathrm{e}^{-}$ 
Equation 2.19 assumes that every coulomb passing through the anode is used to oxidize iron. In reality, some of the charge often goes into oxidizing other available ions (side reactions), such as the oxygen evolution via:

$$
2 \mathrm{H}_{2} \mathrm{O}_{(\mathrm{l})} \rightleftharpoons \mathrm{O}_{2(\mathrm{~g})}+4 \mathrm{H}^{+}+4 \mathrm{e}^{-}
$$

The current efficiency, $\phi$, is often invoked to describe the fraction of coulombs supplied that are actually used to oxidize iron:

$$
\phi=\frac{w_{e x p}}{w_{t h}}
$$

where $w_{\text {exp }}(\mathrm{mg})$ is the measured weight of iron dissolved. $w_{\text {exp }}$ is determined experimentally by measuring the final iron concentration in solution or the difference between electrode weight before and after applied voltage. Both methods fail to take into account the buildup of an active oxide layer on the electrode surface which does not break into solution. An active layer on the electrode surface will lead Equation 2.21 to underestimate the actual iron dissolved by an amount proportional to the thickness of the layer, thereby slightly underestimating the current efficiency. Assuming that $\phi$ is accurate, the actual iron dissolved $w$ becomes:

$$
w=w_{t h} \cdot \phi
$$

Once in solution, the iron ions immediately hydrolyze (within milliseconds) to form (depending on $\mathrm{pH}$ ) a combination of $\mathrm{FeOH}^{+}, \mathrm{Fe}(\mathrm{OH})_{2}^{0}$, and $\mathrm{Fe}(\mathrm{OH})_{3}^{-}$in the case of $\mathrm{Fe}^{2+}$, or $\mathrm{FeOH}^{2+}, \mathrm{Fe}(\mathrm{OH})_{2}^{+}, \mathrm{Fe}(\mathrm{OH})_{3}^{0}$, and $\mathrm{Fe}(\mathrm{OH})_{4}^{-}$in the case of $\mathrm{Fe}^{3+}$. $\mathrm{Fe}[\mathrm{II}]$ species may either precipitate out as $\mathrm{Fe}[\mathrm{II}](\mathrm{hydr})$ oxides and $\mathrm{FeCO}_{3(\mathrm{~s})}$ or oxidize and precipitate to form $\mathrm{Fe}[\mathrm{III}]($ hydr)oxides. Fe[III] species may precipitate directly into 
Fe[III](hydr)oxides. The exact combination of end products is a sensitive function of the potential, $\mathrm{pH}$, and concentration of various species in solution. The resulting phase may be a meta-stable amorphous or freshly-precipitated crystalline mineral (Arienzo et al., 2002). The iron (hydr)oxides produced are sparingly soluble at neutral pH (e.g. HFO, $k_{s p}=10^{-38}$ (Martin \& Kempton, 2000).

The complete generation of iron (hydr)oxides by an iron anode in electrocoagulation is often summarized by the following two mechanisms (Mollah et al., 2004; Daneshvar et al., 2007; Yildiz et al., 2007; Endyuskin et al., 1983):

- Mechanism 1

$$
\begin{gathered}
4 \mathrm{Fe}_{(\mathrm{s})} \rightleftharpoons 4 \mathrm{Fe}_{(\mathrm{aq})}^{2+}+8 \mathrm{e}^{-} \\
4 \mathrm{Fe}_{(\mathrm{aq})}^{2+}+10 \mathrm{H}_{2} \mathrm{O}_{(\mathrm{l})}+\mathrm{O}_{2(\mathrm{~g})} \rightleftharpoons 4 \mathrm{Fe}(\mathrm{OH})_{3(\mathrm{~s})}+8 \mathrm{H}_{(\mathrm{aq})}^{+}
\end{gathered}
$$

Cathode:

$$
8 \mathrm{H}_{(\mathrm{aq})}^{+}+8 \mathrm{e}^{-} \rightleftharpoons 4 \mathrm{H}_{2(\mathrm{~g})}
$$

Overall:

$$
4 \mathrm{Fe}_{(\mathrm{s})}+10 \mathrm{H}_{2} \mathrm{O}_{(\mathrm{l})}+\mathrm{O}_{2(\mathrm{~g})} \rightleftharpoons 4 \mathrm{Fe}(\mathrm{OH})_{2(\mathrm{~s})}+4 \mathrm{H}_{2(\mathrm{~g})}
$$

- Mechanism 2:

Anode:

$$
\begin{gathered}
\mathrm{Fe}_{(\mathrm{s})} \rightleftharpoons \mathrm{Fe}_{(\mathrm{aq})}^{2+}+2 \mathrm{e}^{-} \\
\mathrm{Fe}_{(\mathrm{aq})}^{2+}+2 \mathrm{OH}_{(\mathrm{aq})}^{-} \rightleftharpoons \mathrm{Fe}(\mathrm{OH})_{2(\mathrm{~s})}
\end{gathered}
$$


Cathode:

$$
2 \mathrm{H}_{2} \mathrm{O}_{(\mathrm{l})}+2 \mathrm{e}^{-} \rightleftharpoons \mathrm{H}_{2(\mathrm{~g})}+2 \mathrm{OH}_{(\mathrm{aq})}^{-}
$$

Overall:

$$
\mathrm{Fe}_{(\mathrm{s})}+2 \mathrm{H}_{2} \mathrm{O}_{(\mathrm{l})} \rightleftharpoons \mathrm{Fe}(\mathrm{OH})_{2(\mathrm{~s})}+\mathrm{H}_{2(\mathrm{~g})}
$$

In both mechanisms, hydrogen evolution occurs at the cathode and $\mathrm{Fe}(\mathrm{OH})_{3}$ is the assumed iron (hydr)oxides end product. A schematic representation of the EC arsenic removal process is shown in Figure 2.1. As stated above, the type of iron (hydr)oxides actually produced at the electrode is a sensitive function of potential, $\mathrm{pH}$, and concentration of various species in solution. A Pourbaix diagram is often used to show the dominant species in equilibrium as a function of potential and $\mathrm{pH}$ for a given aqueous concentration. Figure 2.2 shows a simplified Pourbaix diagram for iron (assuming only iron is present in solution) at a given aqueous iron concentration. To demonstrate the complexity of a real system, imagine the entire Pourbaix diagram shifting slightly as the concentration of aqueous iron increases through continuous dissolution of the electrode (Equation 2.19). Further imagine that the electrode potential changes (Equation 2.10) as concentrations of reduced and oxidized species shift. The $\mathrm{pH}$ near the electrode may also change as electrochemical and chemical reactions occur and $\mathrm{OH}^{-}$ions produced at the cathode migrate to the anode in the applied electric field. Recall also that the Pourbaix diagram represents thermodynamic equilibrium, which may or may not be reached at a given potential depending on the rates of charge-transfer, mass-transfer, and chemical reactions in the system. Solids can precipitate and exist for hours to hundreds of years without reaching thermodynamic equilibrium (meta-stable states). Finally, recall that 
the Pourbaix diagram is a function of the entire solution composition, including other co-occuring solutes that may or may not be electrochemically active at a given potential. For all of these reasons, the dominant type of iron (hydr)oxides species produced is extremely difficult to predict.

All major iron (hydr)oxides have been identified in the corrosion products of iron and steel (Cornell \& Schwertmann, 2000). In some cases, the physical placement of corrosion products is more or less random, while in others, the different oxides are arranged in layers. Layer-type rust results from potential or chemical gradients across the oxide film. Such gradients often change with film thickness, leading to rust composition changes with distance from the metal (Cornell \& Schwertmann, 2000). Arsenic adsorption onto ZVI is thought to primarily occur on the oxide film forming around iron filings (Melitas et al., 2002b). In EC, it is not obvious whether arsenic removal is due to adsorption to an iron (hydr)oxides film formed on the anode (which may or may not break off into solution), or adsorption to iron (hydr)oxides formed in solution. Pinisakul et al. (2002) recovered $65-85 \%$ of spiked arsenic from the sludge present in bulk solution after electrocoagulation experiments (initial $\mathrm{pH} 3-10.5$ ) and only $0.03-1.1 \%$ was found adsorbed to the electrode plates and reactor walls, suggesting that adsorbed arsenic does not remain on the electrode. The iron (hydr)oxides produced will also age on timescales of minutes to days, tending to become more crystalline (see Section 2.3). Thus adsorption may occur on slightly more aged iron (hydr)oxides towards the end of electrolysis that at the beginning. 


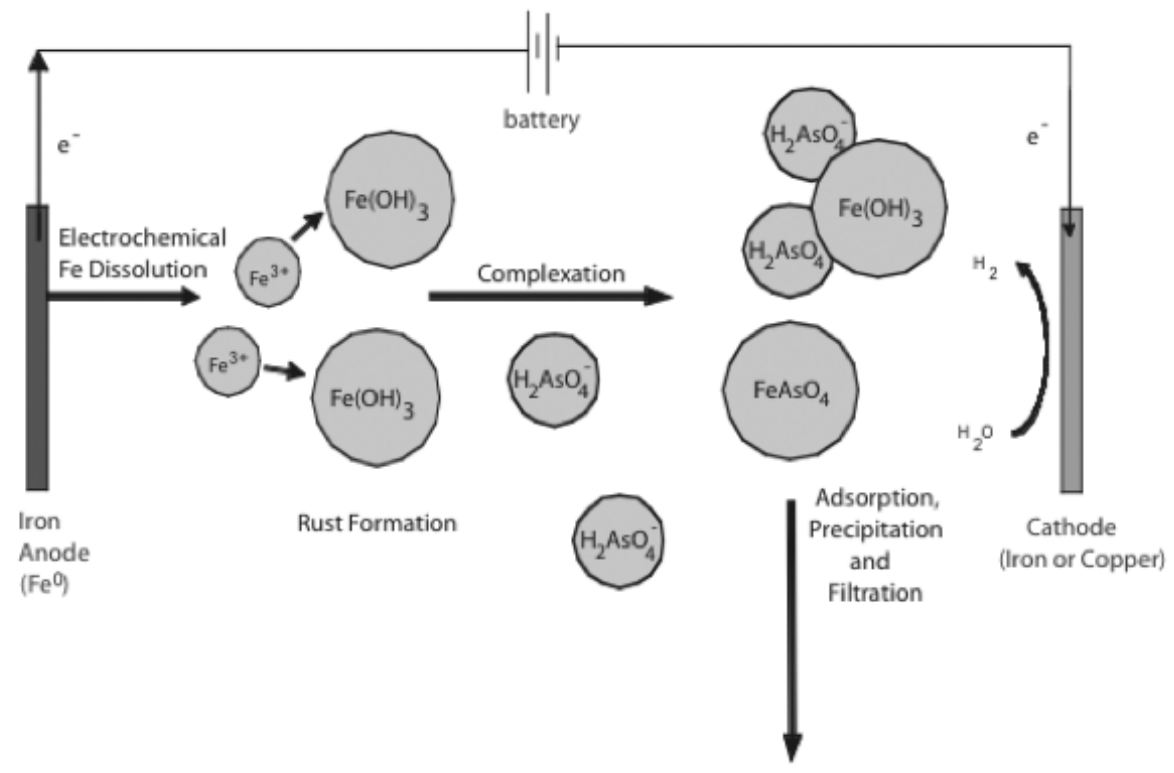

Figure 2.1: Schematic representation of the removal of arsenate ions from solution via EC. For this schematic, $\mathrm{Fe}^{3+}$ ions are dissolving from the electrode, though $\mathrm{Fe}^{2+}$ ions may dissolve as well. In addition, $\mathrm{Fe}(\mathrm{OH})_{3}$ is used to represent the precipitating iron (hydr)oxides, though the actual composition of iron (hydr)oxides formed may be quite complex (see text). 




Figure 2.2: Pourbaix diagram for iron. Assumes iron is the only species present. Eh is the equilibrium potential relative to a standard hydrogen electrode (adapted from Hem (1961)) 
Though the exact composition of iron (hydr)oxides end products is difficult to predict, the overpotential $\eta$ at the anode/solution interface is clearly one of the controlling variables. The overpotential can be adjusted by changing the applied cell potential $E_{a p p l}$ (Equation 2.15). However, in EC it is often desirable to run in galvonostatic mode, in which the current is kept constant through continuous small adjustments of $E_{a p p l}$. In this case, the overpotential is controlled by the current density $j$ via Equation 2.14. Some drifting of $\eta$ may occur during the process to maintain a constant current as $C_{O}(0, t)$ and $C_{R}(0, t)$ evolve with time, potentially leading to a shift in the thermodynamically favorable composition of ironox during the process. However, since $j$ is exponentially dependent on $\eta$, but linearly dependent on concentration, the drift is likely to be relatively small.

Two important parameters used to control EC are thus the current density $j$, which determines the composition of iron (hydr)oxides end products, and the iron dosage $w$ which determines the quantity of iron (hydr)oxides end products. As seen in Equations 2.19 and $2.22, w$ is determined by the operating current $i$, the current processing time $t_{p}$, and the current efficiency $\phi$. Since $\phi$ can only be determined experimentally, the charge density $q$ is often cited instead of $w$, where $q(\mathrm{C} / \mathrm{L})$ is given by:

$$
q=\frac{i t_{C P}}{V}
$$

$i=$ operating current $(\mathrm{A})$

$t_{C P}=$ current processing time $(\mathrm{s})$

$V=$ active solution volume $(\mathrm{L})$ 
If the active solution volume changes during the current processing time, for example as aliquots are removed for sampling, the charge density must be calculated using:

$$
q=\sum_{i} \frac{i \Delta t_{C P i}}{V_{i}}
$$

$i=$ operating current $(\mathrm{A})$

$\Delta t_{c p i}=$ duration of current processing time during which the active volume is $V_{i}$ (s)

$V_{i}=$ active solution volume for duration $\Delta t_{p i}(\mathrm{~L})$

where the total current processing time is:

$$
t_{C P t o t}=\sum_{i} \Delta t_{C P i}
$$

The processing time $t_{C P}$ is often considered important only to the extent that it affects $q$. However, it is possible for $t_{C P}$ to affect the EC process independently of $q$ if coagulation and aggregation are significantly enhanced by the EC process. In this case, more arsenic could be removed per mg of iron (i.e. per unit of $q$ ) when $t_{C P}$ is longer simply because larger particles are more easily separated from the water than smaller ones. In addition, a longer processing time gives the arsenic more time to complex with the continuously generated iron (hydr)oxides, which can be significant if the water is filtered immediately after EC treatment (i.e. with no additional complexation time after the current is turned off). Due to these potential effects, $t_{C P}$ should be considered an independently significant operating parameter controlling the EC process, along with $j$ and $q$.

The electrode surface area to treatment volume ratio, $A / V$, could also be an important controlling EC variable. The electrode area influences current density, posi- 
tion of cation dosing, as well as bubble production and bubble path length (important parameters for electroflotation). However, if filtration is used in place of electroflotation (making the bubble efficiency less important), and the solution is thoroughly and rapidly mixed (leading to even cation dosing regardless of the electrode size and position), then the electrode area should only effect the arsenic removal capacity (mg-As-removed/C) to the extent that it affects current density. Thus, with respect to arsenic removal capacity, it should not be considered a controlling EC variable separate from the current density. Note however that the $A / V$ ratio could separately affect the time required to achieve a given dosage $q$. Rearranging the charge density equation (2.31) in terms of $j$ and solving for $t_{C P}$, one gets:

$$
t_{C P}=\frac{q}{j(A / V)}
$$

Thus, although $A / V$ may not significantly affect the quantity of charge $q$ required to remove $n \mu \mathrm{g} / \mathrm{L}$ of arsenic (the arsenic removal capacity), it may affect the amount of time $t_{C P}$ required to achieve a given dose $q$. This effect was noted by Mameri et al. (1998) while studying the effect of $A / V$ on fluoride removal from Saharan groundwater using aluminum electrodes (a similar process in which removal is based on adsorption of fluoride to electrochemically generated aluminum hydroxides). They reported that minimum processing time required to reduce fluoride concentrations a given amount at a fixed current density decreased as $A / V$ increased, exactly the relation expected by Equation 2.34. They also reported that beyond a value of $A / V=28.7 \mathrm{~m}^{2} / \mathrm{m}^{3}$, no significant effect of $A / V$ on the processing time was observed, suggesting that additional effects may come into play (in the case of Mameri et al. (1998), these additional effects 
could be due to the use of electroflotation).

Equation 2.34 has additional significance as well. It points out that the EC operating parameters $q, j$, and $t_{C P}$, each of which may have distinct control over the arsenic removal capacity, are not completely independent of each other. They can only be adjusted independently if $A / V$ is used as a compensatory variable. $A / V$ in turn is limited by the physical constraints of the electrochemical cell and the extent to which the assumption that $A / V$ does not affect the arsenic removal capacity is valid.

\subsubsection{As[III] oxidation in EC}

One of the major advantages of using EC to remediate arsenic is the potential for a net oxidation of $\mathrm{As}[\mathrm{III}]$ species in the water to $\mathrm{As}[\mathrm{V}]$, which binds more readily to the ironox produced by corrosion at neutral $\mathrm{pH}$ (see Section 2.4.4). Water treatment techniques using chemical addition frequently have to add an oxidizing step before removing As[III] (Arienzo et al., 2002), which can degrade water quality and add to cost. The equilibrium potential for the $\mathrm{As}[\mathrm{III}] / \mathrm{As}[\mathrm{V}]$ redox couple depends on the relative concentrations of $\mathrm{As}[\mathrm{III}]$ and $\mathrm{As}[\mathrm{V}]$. It can also depend on whether the reaction occurs to bound or aqueous arsenic species (Melitas et al., 2002a). The reaction will take place very close to the electrodes, where the $\mathrm{pH}$ can be quite different from the bulk solution due to other reactions taking place there and the migration of $\mathrm{OH}^{-}$ions produced at the cathode. Oxidation of arsenic at the cathode can proceed according to:

$$
\mathrm{H}_{3} \mathrm{AsO}_{3}+\mathrm{H}_{2} \mathrm{O} \rightleftharpoons \mathrm{H}_{3} \mathrm{AsO}_{4}+2 \mathrm{H}^{+}+2 \mathrm{e}^{-}
$$


Reduction of As[V] back to As[III] can occur at the cathode as well. If the cathode is not separated from the anode, net oxidation can only occur if oxidized As[III] ions adsorb to ironox as As[V] before migrating to the cathode. Since As[V] tends to exist as negatively charged oxyanions at neutral $\mathrm{pH}$ (see Section 2.2), it will tend to drift away from the cathode, aiding net oxidation of As[III]. Predominant As[III] species are neutral at neutral $\mathrm{pH}$ will not be pulled by the applied electric field.

As[III] oxidation could also occur in parallel with Fe[II] oxidation due to dissolved $\mathrm{O}_{2}$ (see Section 2.4.3 for more details). Hug \& Leupin (2003) have found this reaction to contribute to $\mathrm{As}[\mathrm{III}]$ removal via zero-valent iron (ZVI).

Net oxidation of As[III] during EC was inferred by Kumar et al. (2004) who measured an initial increase in As[V] concentration during EC on Indian tap water spiked only with As[III]. They attributed the higher removal of As[III] seen in EC relative to the chemical addition of Fe[III] salts to As[III] oxidation. Gomes et al. (2007) verified some oxidation of As[III] to As[V] during EC using X-ray Photon Spectroscopy (XPS). Pinisakul et al. (2002) inferred oxidation of As[III] to As[V] due to the presence of $\mathrm{Fe}_{4} \mathrm{As}_{2} \mathrm{O}_{11}$ in precipitated sludge when only $\mathrm{As}[\mathrm{III}]$ was present in the original solution.

One research group measured $\mathrm{AsH}_{3}$ gas produced at the cathode during electrocoagulation (Pinisakul et al., 2002). The presumed cathodic reaction was:

$$
\mathrm{HAsO}_{2}+6 \mathrm{H}^{+}+6 \mathrm{e}^{-} \rightleftharpoons \mathrm{AsH}_{3(\mathrm{~g})}+2 \mathrm{H}_{2} \mathrm{O}
$$

Between 11-16\% of the arsenic removed was attributed to formation of $\mathrm{AsH}_{3}$ gas. The redox equilibrium potential for Equation 2.36 is $E^{0}=-0.35 \mathrm{~V}$ relative to a standard hydrogen electrode, only slightly less negative than the equilibrium potential of hydro- 
gen evolution (Equation 2.25) with $E^{0}=-0.88 \mathrm{~V}$ (Pinisakul et al., 2002). No other investigations reviewed here have attributed arsenic removal via EC to the formation of arsine gas.

\subsubsection{Arsenic removal mechanism using EC}

The mechanism of arsenic removal in EC is to produce complexes with arsenic that are large enough to be separated from water through either filtration or sedimentation and decantation. The mechanism of complex formation is not completely known. One possible process is adsorption of arsenic oxyanions to electrochemically generated iron (hydr)oxides. Colloidial arsenic-iron (hydr)oxides complexes may form and then aggregate, or arsenic may adsorb to surface of aggregated iron (hydr)oxides. A second possible process is the precipitation of an insoluble arsenic-iron phase (such as ferric arsenate). Surface precipitation of an arsenic-iron phase could also occur on the surface of aggregated iron (hydr)oxides. Other processes that may occur include electrochemically enhanced coagulation of colloidal arsenic oxyanions or even occlusion, which is the entrapment of contaminants in the interior of growing particles. As[III] removal may be enhanced by oxidation to As[V] followed by complexation.

\subsubsection{Arsenic removal studies using EC}

EC has been studied in bench scale reactors to remove arsenic from wastewater (Hansen et al., 2007, 2006; Balasubramanian \& Madhavan, 2001; Gomes et al., 2007) and potable water (Pinisakul et al., 2002; Kumar et al., 2004; Parga et al., 2005b; Arienzo 
et al., 2002). Wastewater studies have demonstrated the ability of EC to reduce influent arsenic concentrations of $100 \mathrm{ppm}$ to below 2 ppm at low initial pH (pH 1-2) using mild steel electrodes with (Hansen et al., 2006) and without (Balasubramanian \& Madhavan, 2001) additional air injection to facilitate oxidation of $\mathrm{Fe}^{2+}$ to $\mathrm{Fe}^{3+}$. Hansen et al. (2006) operated at a current density of $j=12 \mathrm{~mA} / \mathrm{cm}^{2}$ and (Balasubramanian \& Madhavan, 2001) at $j=5$ and $12.5 \mathrm{~mA} / \mathrm{cm}^{2}$. Gomes et al. (2007) used iron plate electrodes to reduce $13.4 \mathrm{ppm}$ As[III] to less than $50 \mathrm{ppb}$ in spiked salt water $\left(j=3\right.$ and $\left.30 \mathrm{~mA} / \mathrm{cm}^{2}\right)$. Ahmed Basha et al. (2008) lowered arsenic concentrations of $2000 \mathrm{ppm}$ in acidic $(\mathrm{pH}<1)$ copper smelting effluent to $100 \mathrm{ppm}$ using EC to reduce arsenic to arsine $\left(\mathrm{AsH}_{3}\right)$ as well as reduce co-occuring sulphate to a species that will readily form insoluble arsenic sulphides $\left(j=20\right.$ and $\left.40 \mathrm{~mA} / \mathrm{cm}^{2}\right)$. EC has also been used to reduce arsenic concentrations to levels acceptable in potable water. Arienzo et al. (2002) used EC with iron and the addition of $10-500$ ppm $\mathrm{H}_{2} \mathrm{O}_{2}$ to reduce $2.5 \mathrm{ppm} \mathrm{As}[\mathrm{III}]$ in spiked tap water to less than $10 \mathrm{ppb}$ for $\mathrm{pH} 3.5-9.5\left(j=0.4 \mathrm{~mA} / \mathrm{cm}^{2}\right)$. Parga et al. (2005a) used EC with carbon steel and air injection to reduce $2 \mathrm{ppm}$ samples to less than $2 \mathrm{ppb}$ (initial $\mathrm{pH} 2.86$ ) and successfully reduced arsenic in well water from La Comarca Lagunera, Mexico from initial values of 25-300 ppb to less than $2 \mathrm{ppb}$ under continuous flow at a rate of $2500 \mathrm{~m}^{3}$ /day $\left(j=3.7-4.6 \mathrm{~mA} / \mathrm{cm}^{2}\right)$. Kumar et al. (2004) reduced $2 \mathrm{ppm}$ of As[V] and As[III] using EC with iron in spiked Indian tap water to less than 10 ppb at pH 6-8 after about 100 $\mathrm{C} / \mathrm{L}$ was passed $\left(j=0.65\right.$ and $\left.1.53 \mathrm{~mA} / \mathrm{cm}^{2}\right)$. Arsenic removal is generally attributed to oxidation of $\mathrm{As}[\mathrm{III}]$ followed by adsorption of $\mathrm{As}[\mathrm{V}]$ to iron (hydr)oxides in solution. Different electrode materials were compared by arsenic removal capacity in sev- 
eral investigations. Gomes et al. (2007) looked at combination Al-Fe electrodes (polarity reversed every 15 minutes) compared to $\mathrm{Fe}-\mathrm{Fe}$ and $\mathrm{Al}-\mathrm{Al}$ electrodes. They found similar removal capacity for Al-Fe and Fe-Fe electrodes, with significantly less removal capacity using Al-Al electrodes alone. Kumar et al. (2004) looked at monopolar aluminum, iron, and titanium anodes and found removal efficiency increased in the sequence aluminum $<$ titanium $<$ iron. Iron has consistently been found to be the most efficient material for arsenic removal.

Notably, few of the above studies stress or monitor any additional complexation time following the electrolysis step in EC. Arienzo et al. (2002) mentions that the postelectrolysis solution is allowed to settle before filtration, but no time is given. Hansen et al. (2006) discuss continuous flow reactors that include a basin for precipitates to settle and cite a distinct current processing time (9.4 minutes) and sedimentation time (80 minutes), but the settling basin is not separate from the electrolysis chamber. Many EC studies do not mention the filtration or separation step at all (Balasubramanian \& Madhavan, 2001; Kumar et al., 2004; Parga et al., 2005b; Mameri et al., 1998), though filtration is implied by the theory stated. In these cases, filtration is assumed to be immediately following electrolysis. Thus there is no study attempting to separate of the effect of iron (hydr)oxides dose (controlled by the electrolysis or current processing time, $t_{C P}$ ) from the effect of arsenic-iron (hydr)oxides complexation time on arsenic removal efficiency in EC. 


\subsubsection{Kinetics of EC}

No study of arsenic removal via EC reviewed here fit data to a kinetic model. Many studies reported that initial arsenic removal was rapid, followed by a slower phase (Pinisakul et al., 2002; Kumar et al., 2004; Balasubramanian \& Madhavan, 2001). Pinisakul et al. (2002) treated initial arsenic concentrations of 0.5-5 ppm and $\mathrm{pH} 3-10.5$. They report that of the arsenic removed at the end of 20 minutes, $99 \%$ had been removed in the first 2 minutes. Kumar et al. (2004) reports $50-60 \%$ of the removal seen after 60 minutes occurred within the first 5 minutes (initial As 2 ppm, pH 6-8). Balasubramanian \& Madhavan (2001) reported a sharp drop in removal rate that eventually approaches a constant value (initial As $100 \mathrm{ppm}$ ). All attribute the initial rapid removal to the abundance of arsenic oxyanions available initially compared to later times.

Mameri et al. (1998) obtained good fits for fluoride removal using EC with aluminum electrodes to a first order model of the form:

$$
\frac{d C}{d t}=-K_{i} C
$$

where $t, C$, and $K_{i}$ are the time, concentration, and kinetic constant respectively. The kinetic constant depends on the current density and temperature. This model fit well over a current density range of $2.89-28.9 \mathrm{~mA} / \mathrm{cm}^{2}$ at $20^{\circ} \mathrm{C}$, confirming that defluoridation follows an exponential law with time. Mameri et al. (1998) attributed the first order behavior to the dual phenomena leading to fluoroaluminum complexes in EC: first the formation of aluminum hydroxide from $\mathrm{Al}^{3+}$ ions on the electrodes, and second the formation of fluoroaluminum complexes by fluoride adsorption on aluminum hydroxide. 
One expects that analogous processes must occur to produce arsenic-iron (hydr)oxides complexes. However, additional processes may occur as well, including the electrochemical oxidation of $\mathrm{As}[\mathrm{III}]$ to $\mathrm{As}[\mathrm{V}]$ and the oxidation of $\mathrm{Fe}^{2+}$ to $\mathrm{Fe}^{3+}$.

\subsubsection{Characterization of EC products}

Several investigators have studied the electrochemically generated by-products of EC (i.e. the iron (hydr)oxides produced). Pinisakul et al. (2002) used X-ray fluorescence and X-ray diffraction (XRD) to study the dried precipitated sludge produced from EC treatment at constant voltage (electric gradient of $200 \mathrm{~V} / \mathrm{m}$ maintained). They found the sludge to contain $98.29 \%$ maghemite, $\mathrm{Fe}_{2} \mathrm{O}_{3}$, and $0.26 \%$ angelellite, $\mathrm{Fe}_{4} \mathrm{As}_{2} \mathrm{O}_{11}$ plus some arsenic trioxide, $\mathrm{As}_{2} \mathrm{O}_{3}{ }^{2}$. The same group also measured arsine gas, $\mathrm{AsH}_{3}$, during electrolysis (see Section 2.5.3). Parga et al. (2005b) used XRD, scanning electron microscopy (SEM), and transmission Mössbauer spectroscopy (TEM) to find nonstoichiometric magnetite particles, goethite, and amorphous iron oxyhydroxides in the air-dried sludge produced from EC with air injection using carbon steel electrodes (current density $j=3.7-4.6 \mathrm{~mA} / \mathrm{cm}^{2}, \mathrm{pH}=7$ ). Arienzo et al. (2002) characterized the products from EC using XRD to find poorly ordered Fe mineral phases with a dominance of 5-line ferrihydrite, and including no lines of ferric arsenate phases $(j=0.4$ $\mathrm{mA} / \mathrm{cm}^{2}$, pH 6.5). Gomes et al. (2007) used a combination of XRD, Fourier transform infrared spectroscopy (FT-IR), X-ray photon spectroscopy (XPS), SEM, and Mössbauer

\footnotetext{
${ }^{2}$ Pinisakul et al. (2002) noted that $\mathrm{Fe}(\mathrm{OH})_{3}$ could be converted to $\mathrm{Fe}_{2} \mathrm{O}_{3}$ during the ignition of sludge samples at 700, which is a requirement of XRD analysis. They also reported at one point a sludge composition of $98.29 \% \mathrm{Fe}_{2} \mathrm{O}_{3}$ and $0.26 \% \mathrm{As}_{2} \mathrm{O}_{3}$ (replacing angelellite for arsenic trioxide). It is not known which is correct.
} 
spectroscopy to find well crystalline phases such as magnetite, and poorly crystalline phases, such as iron oxyhydroxides and lepidocrocite, along with amorphous or ultrafine particulates in the floc produced by EC with iron electrodes $\left(j=3\right.$ or $30 \mathrm{~mA} / \mathrm{cm}^{2}, \mathrm{pH}$ $=0.64)^{3}$.

\subsection{Chapter Summary}

A basic overview of arsenic in natural waters has been given. The predominant forms of arsenic in natural waters $\left(\mathrm{pH} \mathrm{6-8}\right.$ ) include $\mathrm{H}_{2} \mathrm{AsO}_{4}^{-}$and $\mathrm{HAsO}_{4}^{2-}$ for $\mathrm{As}[\mathrm{V}]$ and $\mathrm{H}_{3} \mathrm{AsO}_{3}$ for $\mathrm{As}[\mathrm{III}]$. Numerous insoluble iron (hydr)oxides have been reviewed which can be formed as iron is introduced to water. Iron oxide minerals tend to form in poorly crystalline states with high specific surface area such as amorphous- $\mathrm{Fe}(\mathrm{OH})_{3}($ sometimes called Ferrihydrite) but, over time, undergo transformations to more crystalline forms such as goethite or hematite.

Adsorption, or surface complexation, is the accumulation of a substance at or near an interface relative to its concentration in the bulk solution. Sorption is a term that encompasses adsorption, absorption, and surface precipitation, distinct from solidsolution formation in which a stoichiometric new phase is formed. Adsorption of arsenic onto ferrihydrite, goethite, and other iron (hydr)oxides has been studied extensively and tends to follow a Langmuir type isotherm and consist of strong inner-sphere binuclear, bidentate complexes. Iron (hydr)oxides tend to have a higher affinity for As[V] than As[III] at the $\mathrm{pH}$ of natural waters, though the affinity order can change depending on

\footnotetext{
${ }^{3}$ Both 3 and $30 \mathrm{~mA} / \mathrm{cm}^{2}$ were used in reported batch experiments, but the paper does not state which experiments were used in sludge product analysis
} 
pH. Arsenate adsorption tends to be maximized near $\mathrm{pH} 4$, while arsenite adsorption tends to be maximized near $\mathrm{pH}$ 6-7. Sorption of arsenic in a lab setting is commonly achieved by either coprecipitation or post-synthesis adsorption (PSA). In coprecipitation, iron (hydr)oxides are formed in the presence of arsenic oxyanions, usually by the chemical addition of ferric salts. In PSA, iron (hydr)oxides are formed prior to the addition of arsenic. Adsorption of arsenic onto zero-valent iron (ZVI) in solution is due to the continuous corrosion of the iron, producing $\mathrm{Fe}^{2+}$ and/or $\mathrm{Fe}^{3+}$ ions which quickly hydrolyze to form iron (hydr)oxides adsorbent.

The kinetics of adsorption onto amorphous ferrihydrite, and many other oxides, includes an initial rapid step, typically between 5-30 min, and is sometimes followed by a slower step during which the adsorption density may increase at a rate of a few percent per day. Coprecipitation experiments producing equivalent amounts of ferrihydrite showed much higher adsorption rates at $\mathrm{pH}$ 7.5-9.0, with $>95 \%$ As $[\mathrm{V}]$ adsorbed after 5 min and approximately twice as much As $[\mathrm{V}]$ adsorbed compared to PSA experiments after 24 hours. However, arsenic can begin to desorb from the copreciptate as the coprecipitate ages, approaching the capacity of PSA. The kinetics of arsenic removal via ZVI depends on the rates of several subprocesses, including the corrosion rate of $\mathrm{Fe}(0)$ (which depends on the DO concentration and $\mathrm{pH}$ ), the rate of formation of ferric hydroxide precipitates, as well as the rate of arsenic adsorption onto the precipitates.

Many inorganic and organic aqueous species can affect arsenic adsorption. They can compete with arsenic directly for available binding sites or indirectly by altering the electrostatic charge at the adsorbent surface. Both processes are influenced by $\mathrm{pH}$, ion 
concentration, and the intrinsic binding affinity of the adsorbent. Phosphate, silicate, and carbonate are known to decrease arsenic adsorption, with the combined effect that is worse than the sum of individual effects. Calcium, magnesium, and sulfate have little to no detrimental effect on arsenic adsorption.

A simple electrochemical cell consists of two electrical conductors (electrodes) separated by an ionic conductor (electrolyte). Numerous processes and factors can affect the transport of charge across the electrode/electrolyte interface. The cell potential must be large enough to compensate for the desired current density (governed by the Butler-Volmer relationship) as well as the ohmic drop across the electrolyte. To minimize the ohmic drop and obtain an isolated measurement of the interface potential, a threeelectrode cell is often used.

Electrocoagulation (EC) is a complicated process in which consumable electrodes supply ions that can be used in situ as coagulants or sorbates. The applied electric field can also aid coagulation and aggregation of particles into flocs. EC using iron can generate either $\mathrm{Fe}^{2+}$ or $\mathrm{Fe}^{3+}$ ions which may oxidize (in the case of $\mathrm{Fe}^{2+}$ ) and quickly hydrolyze to form insoluble iron (hydr)oxides. The exact composition of iron (hydr)oxides end products is a sensitive function of potential, $\mathrm{pH}$, and concentration of various species in solution, making it difficult to predict. However, the potential can be used to control the composition. If the process is galvonostatic (constant current), then the current density $j$ can be used to control the potential within a small range via the Butler-Volmer relationship. Other controlling parameters include the charge density $q$, which measures the number of iron ions dissolved into solution and hence the dosage of 
iron (hydr)oxides, and the current processing time $t_{C P}$ which controls the complexation coagulation time. The parameters $j, q$, and $t_{C P}$ can only be adjusted independently of one another if the electrode surface area to active volume ratio, $A / V$, is adjusted as a compensatory variable.

A major advantage of EC is the ability to oxidize As[III $]$ ions to As[V] ions, which more readily form complexes with iron (hydr)oxides at neutral $\mathrm{pH}$. The mechanism of arsenic complexation in EC is likely adsorption of As[III] and As[V] oxyanions onto iron (hydr)oxides, with some As[III] oxidizing to As[V] before adsorption.

EC has been studied in bench scale reactors to remove arsenic from wastewater and potable water. Iron electrodes have been found to be more efficient that aluminum or titanium electrodes. Few studies stress or monitor any additional complexation time following the electrolysis step in EC, though there is potential to increase efficiency by separating the iron (hydr)oxides dose (set by current processing time) from the complexation time. Many studies report that initial arsenic removal is rapid, followed by a slower phase, though none fit data to a kinetic model. A first order model was fit for fluoride removal with aluminum electrodes, but this model differs from arsenic removal in that there is no analogue to the oxidation of $\mathrm{As}[\mathrm{III}]$ and possibly $\mathrm{Fe}^{2+}$. Several studies have characterized the sludge containing corrosion products produced via EC. Amorphous iron oxyhydroxides, including ferrihydrite, are often found, along with some smaller more crystalline phases. 


\section{Chapter 3}

\section{Chemical and Physical Analysis}

\section{of Arsenic Complexation with}

\section{Iron in ECAR}

\subsection{Introduction}

\subsubsection{Operating parameters in ECAR}

It is clear from Chapters 1 and 2 that the parameters in ECAR - current density, $j$, charge density, $q$, current processing time, $t_{C P}$, mixing time, $t_{M}$, and filtration time, $t_{f i l t}$ - may have significant and potentially competing influences on performance considerations relevant to rural Bangladesh - arsenic removal effectiveness, treatment time, and operating costs. Chapter 2 described how these influences depend on specific properties of the water being treated, such as $\mathrm{pH}$ and the composition of co-occuring 
solutes. To better understand the influences of ECAR parameters on performance criteria in rural Bangladesh, empirical studies must be performed in the specific groundwater environment of Bangladesh. Exploratory studies to understand the cause of parameter influence are also essential to better predict and optimize ECAR performance, specifically with regard to lowering costs (a key consideration for rural Bangladesh).

The parameters of ECAR - $j, q, t_{C P}, t_{M}$, and $t_{f i l t}$ - and the processes they govern are described briefly in Section 1.4.1 and in more detail in Section 2.5.2. It will be assumed that the reader is familiar with these parameters and their potential effects.

To study the influence of parameters on performance, it is important to define measurable performance metrics. Performance goals relevant to rural Bangladesh include effective arsenic removal to levels below the legal Bangladesh limit $(50 \mu \mathrm{g} / \mathrm{L})$ or the WHO limit $(10 \mu \mathrm{g} / \mathrm{L})$, a treatment time within about 6-7 hours (for community scale treatment; see Section 1.4.2), and operating costs that are low enough for clean water to be affordable to those making less than US $\$ 1$ per day (for more discussion see Chapter 1 ). Effective removal for Bangladesh is demonstrated by reducing relevant levels of arsenic in a relevant water matrix to less than the legal Bangladesh or the WHO limit. For this work, arsenic removal capability, or just removal capability, will refer to the ability to reach a predefined low arsenic concentration (usually the Bangladesh or WHO limit) given a specific initial arsenic concentration and water matrix (i.e. synthetic or real Bangladesh groundwater). The metric for treatment time will simply be the treatment time, referring to the total time for ECAR treatment, $t_{\text {treat }}=t_{C P}+t_{M}+t_{\text {filt }}$, where $t_{C P}$ is the current processing time (i.e. the time of current flow through the water 
- see Equation 2.34), $t_{M}$ is the mixing time, and $t_{f i l t}$ is the total time of filtration. A measurable metric for operating cost is more subtle. The term arsenic removal efficiency is often used in literature a metric of dosage and cost, but the definition varies and is not always explicitly given. As discussed in Section 1.4.2, both iron and energy consumption (which determine operating costs) depend on the charge required to reduce arsenic from the initial to the final acceptable level. To act as a metric of operating costs, arsenic removal capacity, or just removal capacity, will be used to refer to the average arsenic removed per coulomb to reach a specified arsenic limit (usually the Bangladesh or WHO limit):

$$
\text { Arsenic Removal Capacity }=\frac{[A s]_{i}-[\text { As }]_{\text {limit }}}{q_{\text {limit }}}
$$

where $q_{\text {limit }}$ is the charge density required to reduce arsenic from initial concentration $[A s]_{i}$ to the limit concentration $[A s]_{\text {limit }}$. Operating costs are inversely related to removal capacity (i.e. higher removal capacity will lead to lower costs), though it should be noted that this does not take into account the effect of operating voltage on energy consumption.

The influence of ECAR parameters on performance metrics and the cause of that influence depends on a broad and diverse set of variables covering multiple disciplines. It is necessary to narrow the scope to understanding processes and variables that are most relevant to rural Bangladesh and that could potentially be manipulated to improve performance. In addition, although electrocoagulation is not new, very few studies exist to understand the underlying process of arsenic removal (see discussion in Section 2.5.5). Thus broad exploratory techniques are useful to help direct more specific 
research in the most relevant directions.

Batch tests are a simple yet effective tool to understand the influence of various parameter sets on arsenic removal capability, treatment time, and removal capacity in the specific groundwater environment of Bangladesh. This common technique involves treating small batches of water in the same initial state with some specific combination of operating parameters and comparing outcomes. Batch tests are limited by the ability to recreate the same initial water state and experimental conditions, though variability can be measured with repeat experiments. They can be designed to isolate the effect of single variables or explore the effect of different relevant variable sets, narrowing down the most relevant variables for further study.

Voltammetry is a sensitive technique used to probe the electrochemical behavior of a system. It involves adjusting the potential of an electrochemical cell and observing the resulting current response or polarization curve (i.e. an $i$-E curve, discussed in Section 2.5.1). Slow scan rates can be used to generate polarization curves that correlate a specific current density to the electrode interface potential in the solution of interest (using a three-electrode cell; described in Section 2.5.1). This scan can be compared to thermodynamic equilibrium potentials to identify the likely electrochemical reactions occurring at the iron anode at different current densities in ECAR. Polarization curves can also be used to gain important information about background processes that may affect energy consumption or arsenic removal capacity by decreasing the current efficiency of iron dissolution (discussed in Section 2.5.2).

Electrochemical impedance spectroscopy (EIS) is a powerful electrochemical 
technique that uses the measured impedance of a system to infer information about underlying reaction pathways or to separate and measure electrical properties of the system, such as electrolyte resistance. EIS provides a more accurate measure of electrolyte resistance than using a conductivity probe because electrolyte resistance depends on the geometry of the area in which the current is carried (to convert conductivity probe measurements to electrical resistance, one must assume that there is a uniform current distribution through a definite electrolyte area - rarely true in an electrochemical cell). Electrolyte resistance is a factor in the energy consumption, and hence operating costs, of ECAR treatment.

\subsubsection{Research Objectives}

The primary goals of this research were to demonstrate the arsenic removal capability of ECAR in the groundwater environment of Bangladesh, and to explore the influences of ECAR parameters on arsenic removal capacity for the purposes of optimizing costs within removal capability and treatment time constraints. Based on previous electrocoagulation research and expectations from electrochemical theory, initial focus was placed on parameters $j$ and $q$ with some exploration of $t_{M}$.

We began by designing a bench-top electrochemical cell and reproducible protocol for ECAR treatment. Two recipes were developed to generate reproducible batches of synthetic Bangladesh groundwater (SBGW) - one version with low phosphate and silicate and one version with average levels of phosphate and silicate. Batch tests were used to look at the effect of $j$ and $q$ on arsenic removal capacity and treatment time in 
the ranges $j=0.02-100 \mathrm{~mA} / \mathrm{cm}^{2}$ and $q=0-175 \mathrm{C} / \mathrm{L}$. Additional batch tests were used to explore the influence of $t_{C P}$ and $t_{M}$. Polarization scans, batch tests, and adsorption experiments with post-synthesis ECAR-generated iron (hydr)oxides adsorbent were used to explore the cause of current density trends in removal capacity, study adsorbent aging and affinity for arsenic, and understand the mechanism of As[III] removal. Information gained was used to suggest initial parameters for ECAR field tests and identify regions of parameter space for further study.

\section{Specific Research Objectives:}

1. Design a laboratory method for ECAR, keeping in mind eventual scale-up.

2. Demonstrate the capability of ECAR to reduce high levels of arsenic ( $>98 \%$ surveyed wells) to below the WHO recommended maximum limit of $10 \mu \mathrm{g} / \mathrm{L}$ in synthetic Bangladesh groundwater constituted to mimic key properties relevant to arsenic removal.

3. Characterize arsenic removal capacity of ECAR with respect to current density, $j$, and charge density, $q$, in synthetic Bangladesh groundwater.

4. Explore the cause of current density trends in arsenic removal capacity and the mechanism of As[III] removal.

5. Identify trends and tradeoffs in removal capacity and treatment time between operating parameters.

6. Identify an optimal range of current density and charge density for Bangladesh, 
taking into account arsenic removal effectiveness, treatment time, and minimization of operating costs.

7. Explore low cost alternatives to membrane filtration in ECAR.

\subsection{Methods}

\subsubsection{Arsenic analysis and arsenic speciation}

Arsenic in solution was measured using Inductively Coupled Plasma Mass Spectroscopy (ICPMS) analysis provided by Curtis \& Tompkins, Ltd (Berkeley, CA). ICPMS combines an inductively coupled plasma as a method of producing ions with a mass spectrometer as a method of separating and detecting the ions. It is one of the most accurate available methods for trace detection of metals. Curtis \& Tompkins (C\&T) follow EPA procedure 6020 (EPA, 1998) with a reporting limit for arsenic of 1 ppb and reported accuracy of $10 \%$. Repeated standard samples sent to C\&T suggest a precision of $<4 \%$. All samples were digested prior to being sent to $\mathrm{C} \& \mathrm{~T}$ using $1 \mathrm{~mL}$ of $10 \% \mathrm{HNO}_{3}$ (Mallinckrodt) per $9 \mathrm{~mL}$ sample. All results received from $\mathrm{C} \& \mathrm{~T}$ were multiplied by a factor of 10/9 to compensate for the dilution. One sample containing only deionized water and $\mathrm{HNO}_{3}$ was sent with each group of samples to measure the current arsenic level in the $\mathrm{HNO}_{3}$ (usually $<1 \mathrm{ppb}$, or below detection limit) to ensure no arsenic contamination from the preservative and to provide a check on C\&T procedures.

Sending samples to C\&T and obtaining results for ICP-MS typically took about

10 days. Immediate $(<10 \mathrm{~min})$ arsenic measurements for internal purposes and some 
field measurements were often performed using a Rapid Arsenic Quick Test (Industrial Test Systems, Inc, SC). The Quick Test is a colorimetric test capable of detecting As[V] and As[III] based on the reduction of arsenic compounds to arsine gas. Reported accuracy is $8-80 \%$ (Abbgy et al., 2002). In general, we found Quick Test measurements to be within $20 \%$ of ICPMS measurements over a range of $0-500 \mathrm{ppb}$ (all samples $>30 \mathrm{ppb}$ were diluted to $<30 \mathrm{ppb}$ for testing). Reported errors on Quick Test measurements are based on the separation between adjacent colorimetric levels on the company-provided color chart (5 - $20 \mathrm{ppb}$ depending on concentration). Most Quick test results were later verified via ICPMS. Thus all arsenic measurements reported here are based on ICPMS unless otherwise stated.

$\mathrm{As}[\mathrm{III}]$ and $\mathrm{As}[\mathrm{V}]$ were separated in water using disposable ion exchange columns (Metalsoft Center, Highland Park, NJ) containing $2.5 \mathrm{~g}$ of aluminosilicate adsorbent capable of retaining As[V] but not As[III] in the pH range 4-9 (Meng \& Wang, 1998). Per manufacturer's instructions, only $50 \mathrm{~mL}$ of effluent was accepted from each cartridge with the first $5 \mathrm{~mL}$ discarded. The cartridges were rated for a maximum total arsenic concentration of $500 \mu \mathrm{g} / \mathrm{L}$, so samples were first diluted with deionized water for concentrations above this range. The flow rate was maintained at $<90 \mathrm{~mL}$ per minute using a plastic syringe with a luer tip. Meng et al. (2001) found that the cartridges removed $98 \%$ of the $\mathrm{As}[\mathrm{V}]$ and less than $5 \%$ of the As[III] in spike and recovery tests within the specified operating range. Lab tests of deionized water spiked with 500 and $400 \mu \mathrm{g} / \mathrm{L} \mathrm{As}[\mathrm{V}]\left(\right.$ as $\left.\mathrm{Na}_{2} \mathrm{HAsO}_{4} * 7 \mathrm{H}_{2} 0\right)$ showed $96 \%$ and $100 \%$ removal respectively (via Quick Test), and similar tests with $100 \mu \mathrm{g} / \mathrm{L} \mathrm{As}[\mathrm{III}]$ (as $\mathrm{NaAsO}_{2}$ ) showed no arsenic re- 
moval, consistent with results of Meng \& Wang (1998). The cartridges require complete filtration of particulate arsenic before use, and so could not be relied upon to accurately measure As[III] in solutions in which rapid precipitation of iron (hydr)oxides was taking place (i.e. within 20 minutes of adding iron to arsenic-layden water at neutral $\mathrm{pH}$ ).

\subsection{2 $\mathrm{pH}$, dissolved oxygen measurements}

All pH measurements in the lab and in the field were performed using a SympHony $\mathrm{Ag} / \mathrm{AgCl}$ sealed gel electrode with a built in thermistor for automatic temperature compensation connected to a VWR SympHony Meter (model SP8OPD). The electrode was permanently filled with a KCL gel and was operated and maintained according to factory instructions. The probe was 2-point calibrated every two days of operation using a pH 7.01 buffer (Orion) and one of either a pH 4.01 or $\mathrm{pH} 10.01$ buffer (Orion).

Dissolved oxygen measurements were performed using a SympHony Dissolved Oxygen Probe containing a polargraphic sensor connected to the SympHony meter described above. The probe was operated and maintained according to factory instructions. Before each daily use, the probe was calibrated in water-saturated air (air calibration was recommended by the manufacturer for both air and aqueous measurements). A second 0-oxygen calibration point is recommended, but not required, for measurements of $<2 \mathrm{mg} / \mathrm{L}$, however this was not routinely performed since the majority of measurements were above this range. Note however that this may slightly increase the error on measurements $<2 \mathrm{mg} / \mathrm{L}$ reported below.

To determine probe errors in a solution of similar ionic strength to synthetic 
Bangladesh groundwater (SBGW), 20 consecutive pH and DO measurements were taken of leftover SBGW (recipe SBGW-1; described fully in Section 3.2.3 below) which was several days old and had reached $\mathrm{pH}$ equilibrium (i.e. the $\mathrm{pH}$ no longer drifted up). The $\mathrm{pH}$ probe was freshly rinsed in $0.1 \mathrm{M} \mathrm{HNO}_{3}$ followed by deionized (DI) water. Both probes were rinsed in DI between each measurement. Average $\mathrm{pH}$ was found to be 9.33 \pm 0.06 , and average DO was found to be $2.77 \pm 0.18 \mathrm{mg} / \mathrm{L}$ with no significant outliers. The values 0.06 and $0.18 \mathrm{mg} / \mathrm{L}$ were adopted as the measurement error on the $\mathrm{pH}$ and DO probe respectively.

\subsubsection{Development of Synthetic Bangladesh Groundwater (SBGW)}

Synthetic Bangladesh groundwater (SBGW) is essential for standardized reproducible work. Preparing and storing SBGW requires attention and care. This section describes these steps in detail to aid future experimenters.

\section{Choosing water parameters}

The country of Bangladesh is approximately the size of Iowa and contains several distinct geological regions with potentially very distinct groundwater chemistry. Arsenic occurs in the groundwater beneath the Halocene floodplains of the Ganges, Brahmaputra and Meghna Rivers in the Bengal Basin (Davies, 1995; Ravenscroft, 2003). Hand-pumped tube wells access groundwater 20-75 m deep (Burgess \& Ahmed, 2006) where lateral flow is very low, creating pockets of relatively similar aquifer chemistry in regions where high arsenic is found. Groundwater of the Bengal Basin is generally of the 
$\mathrm{Ca}^{2+}\left(\mathrm{Mg}^{2+}\right)-\mathrm{HCO}_{3}^{-}$type, with a near-neutral $\mathrm{pH}$, a relatively high level of mineralization (the conductivity is generally $500-1000 \mu \mathrm{S} / \mathrm{cm}$ ) and $\mathrm{HCO}_{3}^{-}$commonly present at several hundred mg/L (Burgess \& Ahmed, 2006). The water is predominantly anoxic and strongly reducing (Burgess \& Ahmed, 2006), though oxygen levels can increase by several $\mathrm{mg} / \mathrm{L}$ as the water is pumped from the tube well and into the open air.

The specific groundwater properties that are key to mimicking ECAR performance in Bangladesh are those which can affect the rate of iron dissolution or those which affect the sorption of arsenic onto iron (hydr)oxides. The pH, water conductivity, presence or absence of oxygen, and ions in high concentrations that are electrochemically active in our operating region could affect iron dissolution. The pH, oxygen level (through its effect on arsenic speciation), and competing co-occuring solutes could affect arsenic sorption onto iron (hydr)oxides.

To prepare synthetic groundwater with ion concentrations mimicking conditions in Bangladesh, we initially followed the composition developed by Petrusevski et al. (2002) for background ions, with exception of Fe and Mn. Fe was left out because the small amount present $(0.03 \mathrm{mg} / \mathrm{L})$ would be overwhelmed by the iron from our sacrificial anode. This first recipe (SBGW-1) is presented in Table 3.1. It is characterized by having low phosphate, no silicate, and high sulfate (to make up for ionic strength).

To understand the importance of interfering ions such as phosphate and silicate (see Section 2.4.6) on arsenic removal, a second water batch was developed. To determine representative values of key water properties for the second batch, the British Geological Survey (BGS) was used (BGS, 2001). The BGS, conducted 1998 - 1999, consists of 
Table 3.1: Synthetic groundwater composition and corresponding mean values and standard deviations of groundwater in Bangladesh, obtained from the BGS database (values for $\mathrm{pH}, \mathrm{As}[\mathrm{III}], \mathrm{As}[\mathrm{III}] / \mathrm{As}_{\text {tot }}, \mathrm{HCO}_{3}^{-}$, and $\mathrm{Cl}^{-}$taken from special Study Areas; all other values were taken from National Survey data, using only wells with As $>10 \mu \mathrm{g} / \mathrm{L}$ for data analysis, and setting values below detection limits to zero). For each solute, the percentage of wells in the BGS database with a concentration $(x)$ less than that in the synthetic groundwater 1 and $2\left(x_{s 1}, x_{s 2}\right)$ is given. Values for $\mathrm{pH}, \mathrm{As}, \mathrm{As}[\mathrm{III}]$, and $\mathrm{As}[\mathrm{III}] / \mathrm{As}_{\text {tot }}$ are measured values across water batches, all other ions are spiked values.

\begin{tabular}{|c|c|c|c|c|c|}
\hline & $\begin{array}{c}\text { SBGW } 1 \\
\text { Low } \mathrm{P}, \text { no } \mathrm{Si}\end{array}$ & $\begin{array}{c}\% \text { of BGS wells } \\
\text { with } x<x_{s 1}\end{array}$ & $\begin{array}{c}\text { SBGW } 2 \\
\text { Ave } \mathrm{P}, \text { Ave Si }\end{array}$ & $\begin{array}{c}\% \text { of BGS wells } \\
\text { with } x<x_{s 2}\end{array}$ & $\begin{array}{c}\text { Bangladesh } \\
\text { Groundwater (BGS) }\end{array}$ \\
\hline $\mathrm{pH}_{\text {initial }}$ & $7.04 \pm 0.42$ & $50^{\mathrm{a}}$ & $7.06 \pm 0.16$ & $47^{\mathrm{a}}$ & $7.05 \pm 0.22$ \\
\hline $\mathrm{As}(\mu \mathrm{g} / \mathrm{L})$ & $581 \pm 75$ & 98 & $556 \pm 29$ & 98 & $129 \pm 155$ \\
\hline $\mathrm{HCO}_{3}^{-}(\mathrm{mg} / \mathrm{L})$ & 239 & $0^{\mathrm{a}}$ & 275 & $6^{\mathrm{a}}$ & $501 \pm 144$ \\
\hline $\mathrm{P}(\mathrm{mg} / \mathrm{L})$ & 0.01 & 5 & 1.3 & 67 & $1.3 \pm 1.5$ \\
\hline $\mathrm{Si}(\mathrm{mg} / \mathrm{L})$ & 0 & 0 & 19.5 & 51 & $19.7 \pm 5.1$ \\
\hline $\mathrm{SO}_{4}^{2-}(\mathrm{mg} / \mathrm{L})$ & 81 & 99 & 8 & 88 & $4.6 \pm 17.4$ \\
\hline $\mathrm{Ca}(\mathrm{mg} / \mathrm{L})$ & 53 & 52 & 61 & 56 & $66 \pm 53$ \\
\hline
\end{tabular}

a Comparison to Special Survey Areas of BGS only (155 tube wells over a small region)

b Data available for 6 water batches only (As[III] was not routinely measured) 
samples from 3534 tubewells from 61 of the 64 districts of Bangladesh and from 433 of the 496 Upazilas. The samples covered approximately $0.03-0.05 \%$ of all tubewells in Bangladesh and is believed to be representative of all wells. In addition, three special study areas were established in the Upazilas of Nawabnanj, Faridpur, and Lakshmipur in which $\mathrm{pH}$, dissolved oxygen, and a wide range of additional trace elements were monitored. These study areas are not considered representative of all wells in Bangladesh, and indeed contain a greater proportion of deep wells than found in Bangladesh as a whole, so statistics from these areas were treated with caution.

To determine the average concentration of trace elements in arsenic-contaminated tube wells, only wells with $[\mathrm{As}] \geq 10 \mu \mathrm{g} / \mathrm{L}$ were used (1485 wells in the full survey, 155 in the special surveys). Relevant ions (aside from arsenic) were chosen as those with average concentrations $>0.3 \mathrm{mg} / \mathrm{L}$ in the full survey (excluding $\mathrm{K}, \mathrm{Mn}$ and Fe). Exceptions include relevant ions only available in the special survey, such as $\mathrm{Cl}^{-}$(known to enhance rusting) and $\mathrm{HCO}_{3}^{-}$(known to compete with arsenic). Average values of relevant ions from the BGS are listed in Table 3.1. In calculating averages, values below the detection limit were set to zero (detection limits and percentage of wells below detection limits are also presented in Table 3.1).

The special Survey Area results can be augmented by smaller surveys over wider and more representative geographic areas. Bhattacharya et al. (2002) surveyed 32 wells from 9 affected districts over a much larger geographical range than the special survey areas of the BGS, covering the full extent of the Ganges, Brahmaputra and Meghna rivers in Bangladesh. They measured $\mathrm{HCO}_{3}^{-}$concentrations of 320-600 mg/L (though 
$22 \%$ of wells had $\left.\mathrm{HCO}_{3}^{-}<300 \mathrm{mg} / \mathrm{L}\right), \mathrm{Cl}^{-}$of $1.9-794 \mathrm{mg} / \mathrm{L}$, and $\mathrm{As}[\mathrm{III}] / \mathrm{As}_{\text {tot }}$ of $67-99 \%$. Electrical conductivity varied from $307-1970 \mu \mathrm{S} / \mathrm{cm}$. These values are consistent with those in the BGS, though they suggest that $\mathrm{HCO}_{3}^{-}<300 \mathrm{mg} / \mathrm{L}$ is not as rare as the BGS suggests and that As[III] may be more prevalent in general than As[V].

The total arsenic concentration chosen for synthetic water was $600 \mu \mathrm{g} / \mathrm{L}$, greater than that found in $98 \%$ of wells surveyed by the BGS (actual measured values tended to be a bit lower, represented in Table 3.1). Of the $600 \mu \mathrm{g} / \mathrm{L}$, half was inserted as arsenite $(\mathrm{As}[\mathrm{III}])$ and half as arsenate $(\mathrm{As}[\mathrm{V}])$ to reflect the fact that both appear together in Bangladesh as well as take into account the difficulty of removing As[III] relative to $\mathrm{As}[\mathrm{V}]$.

The initial $\mathrm{pH}$ of all synthetic batches was set to $7.0 \pm 0.4$ to reflect the nearneutral pH of Bangladesh groundwater. The dissolved oxygen (DO) content of tube well water reported in the BGS (from a special Study Area and wells with As $>10 \mu \mathrm{g} / \mathrm{L}$ ) is $0.16 \pm 0.69$. However the act of pumping the water from the aquifer to the surface and into a bucket raised the oxygen level by $2-3 \mathrm{mg} / \mathrm{L}$. To reflect this, dissolved oxygen content in synthetic water was set to $<3.00 \mathrm{mg} / \mathrm{L}$, though in some select cases experiments were done with up to $4.00 \mathrm{mg} / \mathrm{L}$. Average DO levels for SBGW-1 and SBGW-2 were $2.27 \pm 0.50 \mathrm{mg} / \mathrm{L}$ and $2.91 \pm 0.40 \mathrm{mg} / \mathrm{L}$ respectively. In terms of the presence or absence of oxygen (which may be more important than the actual concentration), oxygen was present for these experiments. Equilibrium DO concentrations in the atmosphere were measured to be $7-9 \mathrm{mg} / \mathrm{L}$. 


\section{A reproducible protocol for synthetic water preparation}

To create synthetic Bangladesh groundwater, stock solutions of arsenate (As[V]) and arsenite $(\mathrm{As}[\mathrm{III}])$ were prepared from $\mathrm{Na}_{2} \mathrm{HAsO}_{4} \cdot 7 \mathrm{H}_{2} \mathrm{O}$ (Sigma) and $\mathrm{NaAsO}_{2}$ (Ricca) fully dissolved in deionized water. Deionized water used for stock As[III] solutions was previously purged with $\mathrm{N}_{2}$ and tightly capped to prevent $\mathrm{As}[\mathrm{III}]$ oxidation during storage. The ratio of $\mathrm{As}[\mathrm{III}]$ to $\mathrm{As}_{\text {tot }}$ was monitored every two weeks. This allowed us to ensure minimal conversion of $\mathrm{As}[\mathrm{III}]$ to $\mathrm{As}[\mathrm{V}]$ - if the ratio was less than .95, the solution was discarded and remade. Phosphate stock solutions were made from $\mathrm{Na}_{2} \mathrm{HPO}_{4} \cdot 7 \mathrm{H}_{2} \mathrm{O}$ (EM Sergeant). Bicarbonate stock solutions were made from $\mathrm{NaHCO}_{3}$ (Baker). Calcium Sulfate stock solutions were made from $\mathrm{CaSO}_{4} \cdot 2 \mathrm{H}_{2} \mathrm{O}$ (MP Biomedicals). A mixed stock solution was made from dissolving appropriate amounts of $\mathrm{MgCl}_{2} \cdot 6 \mathrm{H}_{2} \mathrm{O}$ (Mallinckrodt), $\mathrm{NaCl}$ (Mallinckrodt), and $\mathrm{CaCl}_{2}$ (Merck) in DI water. All chemicals were reagent grade.

To begin a water batch (typically 8 liters), stock solutions (excluding As[III]) were mixed together according to the appropriate recipe for SBGW-1 or SBGW-2 (see Table 3.1) and purged with $\mathrm{N}_{2}$ for approximately 1 hour to reduce the dissolved oxygen content, leaving a clear solution with a $\mathrm{pH}$ of about 8 and $\mathrm{DO}<2.0 \mathrm{mg} / \mathrm{L}$. The $\mathrm{pH}$ was then lowered by bubbling excess $\mathrm{CO}_{2}$ gas (at 3 psi) into the water for 1 min or 4 min, leaving a clear solution at about $\mathrm{pH} 7.0$ or $\mathrm{pH} 6.0$ for SBGW-1 or SBGW-2 respectively. For SBGW-2, silicate was added from a daily prepared stock solution $\left(\mathrm{Na}_{2} \mathrm{SiO}_{3}{ }_{5} \mathrm{H}_{2} \mathrm{O}\right.$ dissolved in $200 \mathrm{~mL}$ deionized water). To prevent any polymerization of the silicate, the alkaline stock solution was rapidly mixed into the slightly acidic synthetic groundwater ( $\mathrm{pH}$ 5.8-6.4 with excess dissolved $\mathrm{CO}_{2}$ ) where it was allowed to equilibrate for at least 
1 hour, or in some cases overnight. For SBGW-2 the pH was then raised to $7.0 \pm 0.4$ by bubbling compressed $\mathrm{N}_{2}$ into the solution (in some cases this was not necessary after the solution sat overnight). Finally, As[III] stock solution was added, resulting in a final total arsenic concentration of $600 \mu \mathrm{g} / \mathrm{L}$. The synthetic groundwater was stored in a loosely covered plastic carboy with $\mathrm{N}_{2}$ continuously supplying the headspace to maintain a low DO level. The DO of synthetic groundwater in the storage container tended to be $<1 \mathrm{mg}$ /L (lowered during rapid bubbling of $\mathrm{CO}_{2}$ ), however decanting the synthetic groundwater for testing and $\mathrm{pH}$ measurement generally caused the DO to rise to 2-3 $\mathrm{mg} / \mathrm{L}$. Water batches were stored for a maximum of 2 days until the $\mathrm{pH}$ of the solution rose outside of the required range ( $\mathrm{pH}$ 6.8-7.2). In some cases, As[III] and As[V] stock solutions were added to synthetic groundwater immediately before testing and stirred for 2-3 minutes before taking initial pre-treatment samples.

\section{Water batch behavior}

While the $\mathrm{As}_{\text {tot }}$ concentration and dissolved oxygen concentration in synthetic groundwater batches remained stable over several days, the $\mathrm{pH}$ and $\mathrm{As}[\mathrm{III}]$ concentration showed change. The $\mathrm{pH}$ tended to drift up by about $0.3 \mathrm{pH}$ units per day, quickly leaving the required range of $\mathrm{pH}$ 6.8-7.2. The As[III] concentration tended to decrease rapidly as well, demonstrated by the ratio of As[III]/Astot over 2-3 days for several water batches of the SBGW-1 type (Figure 3.1). Typical behavior, demonstrated by water batch 38 in Figure 3.1, shows almost complete oxidation of As[III] to As[V] after only 3 days. This is much faster than rate of $\mathrm{As}[\mathrm{III}]$ oxidation due to oxygen alone, which is 
known to be kinetically slow. Cherry et al. (1979) found only $25 \%$ oxidation of As[III] after 6 months in water saturated with pure $\mathrm{O}_{2}$ at $\mathrm{pH} 7$ compared to no oxidation in deoxygenated water. Eary \& Schramke (1990) cite a half-life of 1-3 years for As[III] in water equilibrated with atmospheric oxygen. Complete or near complete oxidation of As[III] within 2 days was observed by Devitre et al. (1991) in the presence of synthetic Fe oxyhydroxides and Mn oxyhydroxides, however neither Fe or Mn are present in SBGW-1 batch before electrochemical treatment. Other known oxidizing agents, such as chlorine, potassium, permanganate, peroxide, ozone, and light are either not present in SBGW or unable to completely oxidize As[III] on this rapid timescale. No significant As[III] oxidation was seen in deionized water spiked to $300 \mu \mathrm{g} / \mathrm{L}$ of As[III] and $300 \mu \mathrm{g} / \mathrm{L}$ As[V] (similar to SBGW) after 8 days of monitoring in the same container used to make SBGW, either in equilibrium with the atmosphere or with a DO $<2.0 \mathrm{mg} / \mathrm{L}$ (maintained through constant $\mathrm{N}_{2}$ purging of the headspace). $75 \%$ oxidation after 5 days was observed (using Quick Test results only) using the SBGW-1 recipe in equilibrium with the atmosphere, with and without excessive bubbling of $\mathrm{CO}_{2}$.

As[III] is known to form complexes with calcite (Roman-Ross et al., 2006). Calcite is oversaturated in SBGW, and its formation would lead to an increase in the $\mathrm{pH}$ over time, as observed. It is possible that calcite could capture some As[III] and settle to the bottom of the water batch storage container, effectively reducing the As[III] concentration. However, this would lead to a decreasing Astot concentration over time, and $\mathrm{As}_{\text {tot }}$ was observed to be stable. Careful studies to discover the cause of the rapid oxidation of As[III] were not pursued in favor of simply cutting off the useful lifetime of 
each water batch at 1 day.



Figure 3.1: The ratio of $\mathrm{As}[\mathrm{III}]$ to total-arsenic $\left(\mathrm{As}_{t o t}\right)$ over a period of 2-3 days for three representative water batches (WB) of the SBGW-1 type. For water batches 38 and 45, no initial (0 day) measurement of As[III] was done, so average As[III]/Astot ratio of monitored water batches ( 8 total) at day- 0 was used with the standard deviation as an error.

\subsubsection{Electrochemical cell}

Two benchtop electrochemical cells were used in experiments, each consisting of a glass beaker containing a pure iron wire $($ diameter $=0.18 \mathrm{~cm})$ anode coiled into a spiral and a copper mesh cathode. The active area of both iron and copper electrodes varied with experiment, ranging from $9-150 \mathrm{~cm}^{2}$ for the iron and $24-150 \mathrm{~cm}^{2}$ for the copper mesh. The iron wire was tested for iron content by Curtis \& Tompkins Lab (Berkeley, CA) using EPA preparation procedure 3050B and testing procedure 6010B and found to contain $10^{6} \mathrm{mg} / \mathrm{kg}$ iron (essentially $100 \%$ iron). Both cells were hooked up in series with an EG\&G model 173 Potentiostat/Galvonostat in galvonostatic mode 
with either a model 376 logarithmic current converter or model 176 current follower (all from Princeton Applied Research). This was in series with a Kiethley model 173A or Fluke model 73111 multimeter set to measure current. The setup for each cell is shown in Figures 3.2 and 3.3.

The first of the two cell configurations was a $850 \mathrm{~mL}$-separated cathode cell shown schematically in Figure 3.2. The 1 liter cell beaker had a diameter of $11 \mathrm{~cm}$. The iron electrode, coiled to a diameter of about $10 \mathrm{~cm}$, was submerged to approximately $4 \mathrm{~cm}$ above the beaker bottom to make room for a magnetic teflon-coated stir bar. A Schott porous glass frit filter, consisting of a glass beaker (diameter $=4.3 \mathrm{~cm}$ ) with a porous glass frit bottom (allowing charge, but very little water to pass), was placed approximately $2 \mathrm{~cm}$ above the anode with the copper cathode placed inside the glass beaker, creating a separated cathode chamber. The top of the glass filter was held above water level to ensure minimal mixing of the cathodic and anodic solutions. Sample water filled both chambers up to a total volume of $850 \mathrm{~mL}$ (approximately $40 \mathrm{~mL}$ in the cathode chamber). Separation of the cathode was intended to shield the main treatment volume from the reduction of $\mathrm{As}[\mathrm{V}]$ to $\mathrm{As}[\mathrm{III}]$ at the cathode and the $\mathrm{pH}$ increase caused by $\mathrm{H}^{+}$reduction to $\mathrm{H}_{2}$ gas. Minimum separation between the cathode and anode was approximately $2.5 \mathrm{~cm}$.

The second cell configuration was a 3 L-single chamber cell shown schematically in Figure 3.3. The cell body was a $3.5 \mathrm{~L}$ glass beaker with a diameter of $15.3 \mathrm{~cm}$. A flat spiral iron electrode (same shape and material used in the $850 \mathrm{~mL}$-separated cathode cell) was submerged $1 / 6$ of the total water height $(13 \mathrm{~cm}$ from the bottom), with the square 
copper mesh electrode placed flat on top. Two layers of refurbished polyvinylidene fluoride hydrophilic membranes with $0.1 \mu \mathrm{m}$ pore size (Millipore; see Section 3.2.5 for refurbishing procedure) were placed between the two electrodes for insulation, leading to an electrode separation of $0.05-0.5 \mathrm{~cm}$ (range varied as the iron electrode coil was not completely flat).

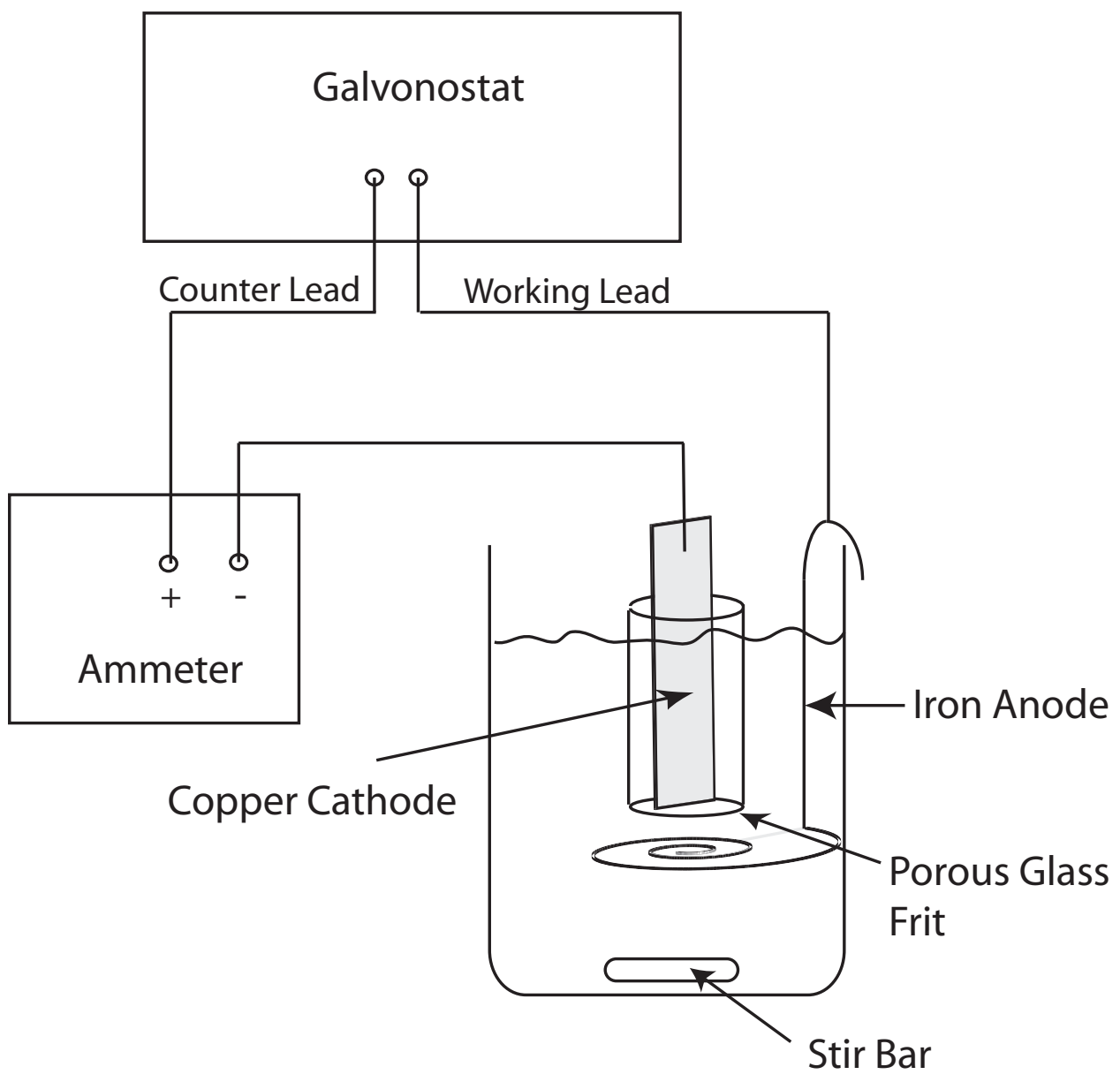

Figure 3.2: Schematic representation of $850 \mathrm{~mL}$-separated cathode electrochemical cell. 


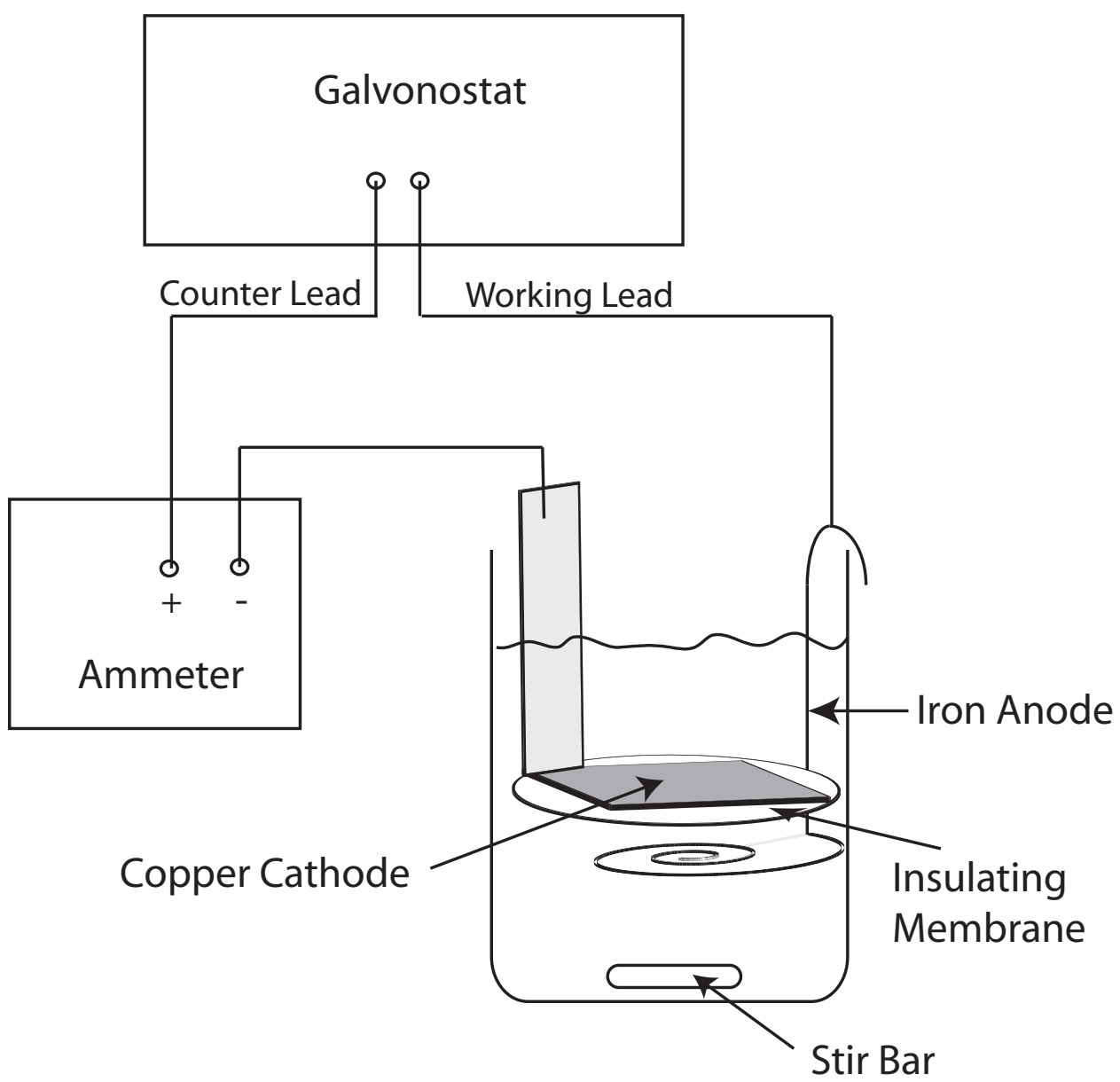

Figure 3.3: Schematic representation of 3 L-single chamber electrochemical cell. 


\subsubsection{Electrochemical Arsenic Remediation Procedure}

ECAR treatment consists of three stages in series; (1) electrochemical dosing, (2) mixing, and (3) filtration. The dosing stage is characterized by electrodes in contact with the contaminated water, as a galvonostatic (constant) current $I$ is passed using an external voltage source. During this stage, iron dissolves in amounts determined by Equation 2.19, forming EGA sludge (i.e. iron (hydr)oxides) in solution. Other processes that may occur during the dosing stage are discussed in Section 2.5.2. During the mixing stage, external voltage is off and electrodes are removed from the water, while the dosed solution is stirred or lightly agitated to facilitate further complexation between the arsenic in the water and EGA as well as coagulation of EGA and/or arsenic-EGA complexes to allow for filtration. In the filtration stage, arsenic-laden EGA is separated from the water using some kind of filtration process.

The basic methods used for each of the three ECAR stages during all lab tests are described below. Specific experiments are fully described by adjustable ECAR operating parameters $q, j, t_{C P}$ and $t_{M}\left(t_{f i l t}\right.$ is generally determined by the filtration process) along with experimental conditions, including the electrochemical cell parameters (designated by $A / V$ and the cell design), and the test solution composition (generally designated by SBGW-1 or SBGW-2). Experiment-specific procedures and/or experimentspecific modifications to the basic method are described in the subsequent sections. 


\section{Dosing method}

The dosing stage began by filling the empty electrochemical cell (i.e. without electrodes $)$ to the appropriate volume $(850 \mathrm{~mL}$ or 3 liters for the $850 \mathrm{~mL}$-separated cathode cell or the 3 L-single chamber cell respectively) with sample water (usually synthetic Bangladesh groundwater). In some cases, the sample water was stored without arsenic. In such cases, one or both of the As[III] and/or As[V] stock solutions were poured into the empty cell beaker first, with the sample water poured on top to facilitate mixing. Initial $\mathrm{pH}$ and $\mathrm{DO}$ measurements were taken in the beaker during mild stirring (via a magnetic stirrer); also facilitating the mixing of As[III $]$ and $\mathrm{As}[\mathrm{V}]$ into the solution. Initial $\mathrm{pH}$ and DO measurements took approximately 2-3 min, during which time the DO tended to rise from $<1.5 \mathrm{mg} / \mathrm{L}$ to $2-3 \mathrm{mg} / \mathrm{L}$. If required, aliquots of solution to measure pre-treatment $\mathrm{As}_{\text {tot }}$ and $\mathrm{As}[\mathrm{III}]$ concentrations were removed from the top using a glass luer tip syringe. If the pre-treatment As[III] concentration was measured, approximately half of the aliquot was immediately filtered through a speciation cartridge.

The electrodes were then inserted and connected according to Figures 3.2 or 3.3. The galvonostat was turned on at $t=0$ (measured by a portable timer) to provide constant current $I$, along with the magnetic stirrer using a low setting (just high enough to create a visible vortex at the top of the cell). Current was monitored using either the Keithley or Fluke digital multimeter (see Section 3.2.4. After a total current processing time $t_{C P}$, current and stirring were turned off and the electrodes were immediately removed, thus completing the dosing stage. Dosed solution was immediately passed into the mixing stage (within 1 minute or less). 


\section{Mixing method}

The mixing stage consisted of stirring the dosed solution in an open beaker for a fixed amount of time $t_{M}$ using a magnetic stirrer. A post-dosage $\mathrm{pH}$ and DO measurement were often taken during the first 2-3 minutes of mixing. Solution would pass from the mixing to the filtration stage within 5 minutes (sometimes waiting for an available filter apparatus).

\section{Filtration method}

Mixed solutions were filtered using a pyrex flask vacuum filter (Kontes) and $90 \mathrm{~mm}$ diameter polyvinylidene fluoride hydrophilic membranes with $0.1 \mu \mathrm{m}$ pore size (Millipore). Filtration of $150 \mathrm{~mL}$ took approximately 4-5 minutes.

Repeated filtration of the filtrate showed no change in arsenic concentration, indicating that no water was leaking around the membrane and all particles $>0.1 \mu \mathrm{m}$ were filtered out in the first pass. Results of filtration with pore sizes up to $0.8 \mu \mathrm{m}$ (discussed in Section 4.3.1) showed no increase in arsenic concentration, also indicating full removal of particles $>0.1 \mu \mathrm{m}$.

Membranes were refurbished one time after use in batch tests by soaking in dilute $\mathrm{HCl}$ and rinsing twice in deionized water. Filtration of mixed water using refurbished membranes yielded the same arsenic concentration (within 5\%) as filtration with new membranes. Refurbished membranes were used to insulate the anode from the cathode in the 3 L-single chamber cell only (see Section 3.2.4) - they were not used for filtration during batch tests. 


\section{Lab practices}

Before treatment, the electrochemical cell beaker was always rinsed in diluted $\mathrm{HCl}$ followed by DI water to remove any buildup of iron (hydr)oxides precipitate or arsenic from previous batch tests. All beakers used for aliquot transport were thoroughly rinsed in DI water. The vacuum filter apparatus was doubly rinsed in DI water between each filtration along with periodic rinses in dilute HCl. Sample vials for ICPMS testing were unused and radiation sterilized by the vendor (BD Biosciences). Sterile gloves were worn for all procedures.

\subsubsection{ECAR batch experiments run 1 - Matrix 1}

\section{Purpose and experimental parameters}

ECAR batch experiments were performed to (1) determine the minimum dose required to reduce arsenic concentrations from $600 \mu \mathrm{g} / \mathrm{L}$ to below the Bangladeshi and/or WHO maximum allowable concentration $(50 \mu \mathrm{g} / \mathrm{L}$ and $10 \mu \mathrm{g} / \mathrm{L}$ respectively $)$ as well as (2) study the effect of current density on arsenic removal capacity. In this case, arsenic removal capacity refers to the amount of arsenic removed per coulomb passed (in $\left.\mu \mathrm{g} / \mathrm{L}-\mathrm{As}_{\text {removed }} / \mathrm{C}\right)$. This round of testing, known as Matrix 1, used SBGW-1 synthetic groundwater and the $850 \mathrm{~mL}$-separated cathode electrochemical cell. Sixteen individual batch tests were performed to cover all combinations of current density $j=0.07,0.3$, 0.7 , and $1.1 \mathrm{~mA} / \mathrm{cm}^{2}$ combined with current processing times $t_{C P}=3,5,11$, and $50 \mathrm{~min}$ (total dose $1.4-390 \mathrm{C} / \mathrm{L}$ ). All tests used one of two flat spiral iron anodes with an active (submerged) area of $100 \mathrm{~cm}^{2}$, leading to a surface-area-to-volume ratio of $0.118 \mathrm{~cm}^{-1}$. 
Each electrode was either sanded with fine sandpaper or rinsed in dilute $\mathrm{HCl}$ followed by deionized water every three batch tests or every day, whichever came first. Initial arsenic was measured within 1 hour of creating each synthetic water batch. In one case, $j=1.1 \mathrm{~mA} / \mathrm{cm}^{2}$ and $t_{C P}=50 \mathrm{~min}$, the initial arsenic was not measured, and the initial arsenic for that test was taken to be the average of all water batches created for this run.

\section{Establishing the mixing time}

In order to set the appropriate mixing time, $t_{M}$, for batch experiments, some initial mixing kinetics tests were performed. In each test, dosed water was mixed for some time with aliquots removed, filtered, and tested for arsenic using the Quick Test (described in Section 3.2.1) every 5 minutes for 25 - 30 minutes. Results for two such tests (kinetics test 1 and 2 ) after dosing at $j=1.1 \mathrm{~mA} / \mathrm{cm}^{2}, q=39 \mathrm{C} / \mathrm{L}, t_{C P}=5 \mathrm{~min}$ are shown in Figure 3.4. In both cases, the arsenic concentration falls rapidly for the first 1015 minutes of mixing, and much more slowly after that. In the case of kinetics test 1 , the concentration seems to stabilize after $t_{M}=15 \mathrm{~min}$. In kinetics test 2 , the concentration drops rapidly for about 5-10 minutes followed by a slow decrease continuing after 25 minutes.

Adsorption of As $[\mathrm{V}]$ onto pre-formed amorphous ferrihydrite at $\mathrm{pH} 8$ is reported to have an initial rapid uptake $(<5 \mathrm{~min})$ followed by a slow uptake for as long as 8 days (Fuller et al., 1993). Coprecipitation experiments producing the same equivalent amount of ferrihydrite have shown rapid uptake $(<5 \mathrm{~min})$ followed by slow uptake for about 24 hours, followed by very slow desorption continuing after 31 days of reaction (Fuller et al., 




Figure 3.4: Fraction of remaining arsenic as a function of mixing time for SBGW-1. Both kinetics tests were performed under identical conditions after a dosing at $j=$ $1.1 \mathrm{~mA} / \mathrm{cm}^{2}, q=39 \mathrm{C} / \mathrm{L}, t_{C P}=5 \mathrm{~min}$, with initial arsenic As $\mathrm{s}_{t o t} \approx 1000 \mu \mathrm{g} / \mathrm{L}$.

1993), though final arsenic removal was still greater than for pre-formed ferrihydrite (see Section 2.4.5 for more discussion). In ECAR, arsenic concentrations at $t_{M}>15 \mathrm{~min}$ either stabilize at a constant value (suggested by kinetics test 1 in Figure 3.4), continue to slowly decrease (at a rate that is below the Quick Test detection limit), or slowly decrease and then begin to increase (as suggested by coprecipitation experiments). If arsenic continues to decrease slowly, then very little is gained past $t_{M}=15$ minutes for a large cost in treatment time (hours to days). If arsenic stabilizes or begins to increase, then nothing is gained and some removal capacity may be lost. Thus $t_{M}$ was chosen to encompass the period of rapid arsenic removal but exclude slow removal past a reasonable treatment time of several hours. Since $t_{M}$ is only one portion of the treatment time, it 
was set at 60 minutes for Matrix 1 batch $\operatorname{tests}^{1}$ to ensure a total treatment time of less than 2 hours.

The mixing kinetics profile in ECAR is likely to change with current processing time, $t_{C P}$. Complexation with arsenic will begin as soon as iron (hydr)oxides begin to form during electrolysis, and more or less of the rapid complexation phase seen in Figure $3.4\left(t_{C P}=5 \mathrm{~min}\right)$ may occur in this time. The kinetics profile may also change with current density if the adsorbent generated by ECAR is different. For these reasons, a check was placed on the $t_{M}$ value of 60 minutes for batch tests with different values of $t_{C P}$ and current density. A $120 \mathrm{~mL}$ aliquot was removed during mixing after $t_{M}=$ $40 \mathrm{~min}$ for each batch test. The aliquot was filtered and tested for arsenic using the Quick Test. If this measurement disagreed with a Quick Test measurement taken at $t_{M}$ $=60$ min by more than $20 \%$ (the approximate precision of the Quick Test), mixing was allowed to continue for an additional 20 minutes to ensure that the rapid complexation phase was complete. For Matrix 1, all Quick Test measurements taken at $t_{M}=40 \mathrm{~min}$ and $t_{M}=60$ min agreed to within $20 \%$.

It should be noted that in kinetics tests, the mixing time was defined as the time between dosage completion (i.e. electrodes removed from water) and pouring out the sample aliquot for filtration. Some additional complexation may have occurred during the 3-4 minutes between the start and end of filtration, leading to an uncertainty in complexation time of about $3 \mathrm{~min}$. This is expected to be more significant during the period of rapid complexation at $t_{M}<10-15 \mathrm{~min}$. It should also be noted that the

\footnotetext{
${ }^{1}$ The only exception was the test at $j=1.1 \mathrm{~mA} / \mathrm{cm}^{2}$ for $t_{C P}=50 \mathrm{~min}$, which has $t_{M}=30 \mathrm{~min}$. In this case, the Quick Test measured $\mathrm{As}_{t o t}=0 \mu \mathrm{g} / \mathrm{L}$ at both $t_{M}=20 \mathrm{~min}$ and $t_{M}=30 \mathrm{~min}$, indicating that rapid complexation was complete.
} 
electrochemical cell and SBGW-1 preparation procedure used for kinetics tests were slightly different than those described above. The iron electrode had the same active area, but was in a 3D coil shape rather than a spiral. The SBGW-1 water used was spiked to $\mathrm{As}_{t o t}=1000 \mu \mathrm{g} / \mathrm{L}$ instead of $600 \mu \mathrm{g} / \mathrm{L}$ (still containing a 50:50 ratio of $\mathrm{As}[\mathrm{III}]$ to $\mathrm{As}[\mathrm{V}])$. The $\mathrm{pH}$ of the water batch was lowered with small amounts of dilute sulfuric acid in place of bubbling $\mathrm{CO}_{2}$ (pre-treatment $\mathrm{pH}$ was 6.85 and 6.93 for kinetics tests 1 and 2 respectively). None of these differences are expected to have any significant effect on the kinetics behavior during mixing.

\section{Replication}

All tests were duplicated, and in some cases repeated 3-5 times. However, later ICPMS results of the pre-treatment sample water showed a greater than expected range of initial $\mathrm{As}[\mathrm{III}] / \mathrm{As}_{\text {tot }}$ ratios for $\mathrm{SBGW}-1$, in some cases falling much below 0.4 or greater than 0.6 (see Section 3.2.3). An extreme initial As[III]/ $\mathrm{As}_{\text {tot }}$ ratio is likely to bias batch test results because, in general, As[III] requires a higher dose to remove than As $[\mathrm{V}]$. Indeed, all replicates with extreme initial As[III]/Astot ratios followed this trend relative to the original tests. In order to maintain a consistent sample, batch tests with a measured $\mathrm{As}[\mathrm{III}] / \mathrm{As}_{\text {tot }}<0.40$ or $>0.6$ were removed. To account for water batches with no measured As[III]/Astot, and given the general trend for As[III]/Astot to decrease with storage time (measured in Figure 3.1), all tests using water batches $>1$ day old were removed as well. This cut left 7 out of 16 total batch tests with only a single measurement (no repeat), 8 out of 16 with two measurements, and 1 test with three 
measurements.

\section{Variability estimates}

To estimate the expected variability between outcomes of replicated batch tests (i.e. batch tests performed using the same ECAR operating conditions to treat water of the same composition), the eight Matrix 1 tests with at least one acceptable repeat measurement were used. Outcomes between "identical" batch tests may differ due to one or more of the following: slight differences in composition between water batches, slight differences in ambient pressure and temperature in the lab (expected to be small), slight differences in initial DO content (measured standard deviation of $0.5 \mathrm{mg} / \mathrm{L}$ between water batches) or initial $\mathrm{pH}$ (standard deviation of $0.42 \mathrm{pH}$ units between water

batches) as well as $\mathrm{pH}$ and DO drift during dosing and mixing (size of drift dependent on current processing and mixing time), differences in the initial spiked arsenic concentration (ICPMS measured standard deviation of total initial arsenic of $75 \mu \mathrm{g} / \mathrm{L}$, or $13 \%$ ) and differences in the initial As[III]/As[V] ratio (ICPMS measured standard deviation of 0.05 , or $10 \%$ ) and finally, uncertainty associated with ICPMS arsenic measurement (quoted as within $10 \%$ by $\mathrm{C} \& \mathrm{~T}$ lab). Many of the above sources of variability have a complicated and likely non-linear effect on the final arsenic concentration measured in a given batch test. Some are likely to cause more variability with increasing final arsenic concentration, while some are expected to cause random variability. The available data set is too small to discern between a linear and non-linear model, and thus a linear model has been chosen to obtain a rough estimate of variability. To remove error 
associated with slightly different initial arsenic concentrations between repeated tests, all arsenic concentrations were normalized to have an initial concentration of $\mathrm{As}_{\text {init }}=$ $580 \mu \mathrm{g} / \mathrm{L}$. Figure 3.5 shows the replicate variability between repeat measurements (i.e. $\left|y_{2}-y_{1}\right| / \sqrt{2}$ for two replicate measurements $y_{1}$ and $\left.y_{2}\right)^{2}$ as a function of the average final arsenic concentration.

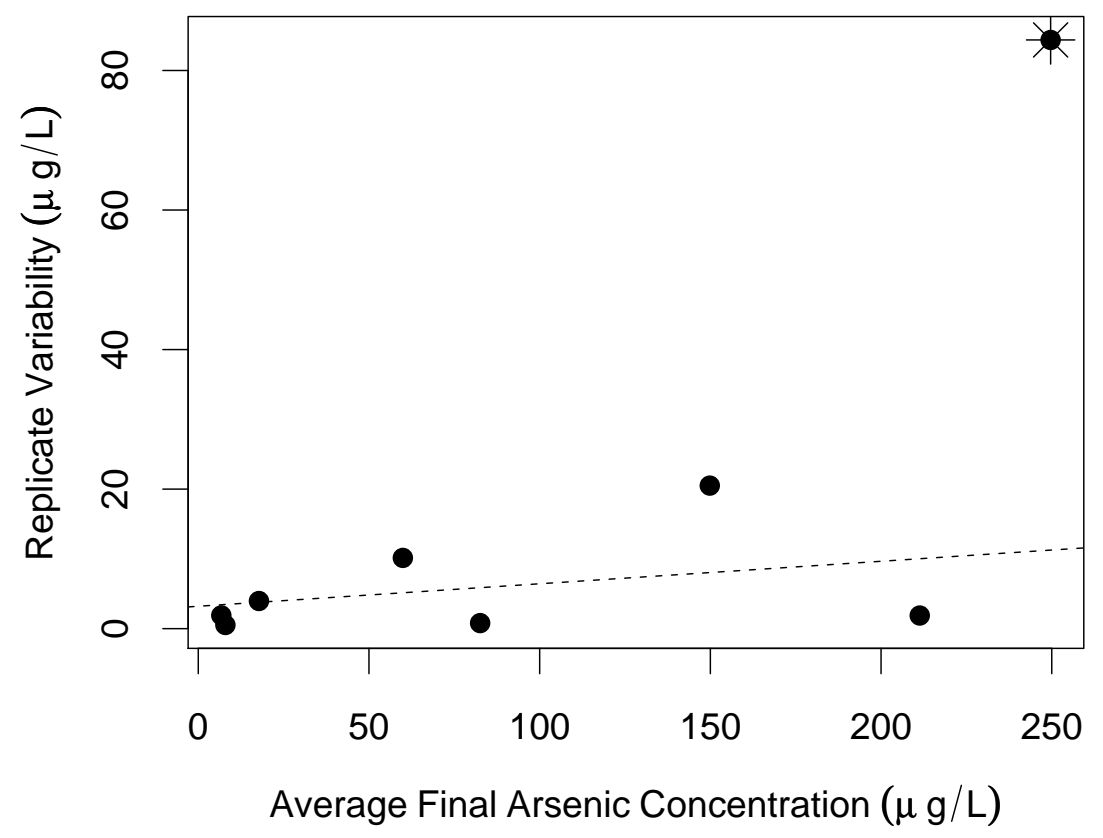

Figure 3.5: Replicate variability between arsenic concentrations measured in identical batch tests (repeat measurements) for Matrix 1 (where repeats are available) as a function of the average final arsenic concentration, $A s_{\text {fave }}$. The starred point has a higher than expected standard deviation, and represents the batch test performed at $j=30$ $\mathrm{mA} / \mathrm{cm}^{2}$ and $t_{C P}=5 \mathrm{~min}$. The dotted line in a linear fit to non-starred points.

Figure 3.5 indicates a trend (as expected) of increasing uncertainty with increasing average arsenic concentration. The trend appears approximately linear with

\footnotetext{
${ }^{2}$ For the one case with three acceptable replicate measurements, the replicate variability is the standard deviation.
} 
the exception of the final point (designated by a star) corresponding to the batch test performed at $j=30 \mathrm{~mA} / \mathrm{cm}^{2}$ and $t_{C P}=5 \mathrm{~min}$. The deviation between tests for this point is $84 \mu \mathrm{g} / \mathrm{L}$, or $34 \%$ of the average final arsenic value, much higher than the deviation measured for nearby points. While this deviation is consistent with highly non-linear behavior, it is also consistent with an unknown procedural error in one or both of the batch tests at $j=30 \mathrm{~mA} / \mathrm{cm}^{2}, t_{C P}=5 \mathrm{~min}$. Attribution to a procedural error is further supported by the fact that the resulting final arsenic concentrations for both batch tests at $j=30 \mathrm{~mA} / \mathrm{cm}^{2}, t_{C P}=5$ min were higher than the final arsenic concentrations at $j=30 \mathrm{~mA} / \mathrm{cm}^{2}, t_{C P}=3 \mathrm{~min}$, despite representing a higher dose. No other set of batch tests violated the trend of a higher dosage leading to a lower final arsenic concentration ${ }^{3}$. For these reasons, a procedural error has been assumed, and all batch tests for $j=30$ $\mathrm{mA} / \mathrm{cm}^{2}, t_{C P}=5 \mathrm{~min}$ were discarded for further analysis.

The remaining points were fit to a linear model, shown in Figure 3.5 as a dotted line. The resulting estimated variability for Matrix 1 points, $\sigma_{M 1}$, as a function of final arsenic concentration, $A s_{f}$, is (in units of $\mu \mathrm{g} / \mathrm{L}$ ):

$$
\sigma_{M 1}=3.2 \pm 4.1+(0.032 \pm 0.039) A s_{f}
$$

The $R^{2}$ value for this fit was quite low, with $R^{2}=0.1192$. In addition, the estimated errors for each parameter in Equation 3.2 are greater than 100\%. However, no better estimate is allowed by the small data set, leading to the adoption of this model over none. The model fit is low compared to the reported ICPMS measurement errors of

\footnotetext{
${ }^{3}$ In one other case $\left(j=110 \mathrm{~mA} / \mathrm{cm}^{2}\right)$, the average of two $t_{C P}=5 \mathrm{~min}$ batch tests resulted in a slightly higher final arsenic concentration than the average of the $t_{C P}=3 \mathrm{~min}$, however the difference was well within the error of ICPMS measurement and so not significant.
} 
$\pm 5 \%$ with a reporting limit of $1 \mu \mathrm{g} / \mathrm{L}$. This could be due to ICPMS error inflation by C\&T or the weakness of the fit.

\section{Experiments with no external voltage applied}

A series of tests were also run to study the arsenic removal capacity of the ironcopper electrode system with no external voltage applied. These tests followed the same procedure as above, but with the galvonostat removed from the circuit (i.e. no external voltage applied). Two batch tests were performed with current processing times $t_{C P}=$ 11 and 50 min. Each test was repeated and the results averaged. The iron electrode was rinsed in dilute $\mathrm{HCl}$ and $\mathrm{DI}$ water to remove rust between each batch test, with the exception of the duplicate $t_{C P}=50$ min test. This test used a pre-rusted iron electrode (rusted by allowing DI water to drip on the iron in air for several days). The measured $\mathrm{As}[\mathrm{III}] / \mathrm{As}_{t o t}$ ratio of the water used was 0.62 , slightly higher that the ratio allowed for other Matrix 1 experiments. The measured current through the electrodes during these tests ranged from $0.67-0.036 \mathrm{~mA}$, usually starting high and progressively decreasing, though in one case the current started low and increased. Two final batch tests were performed with no external voltage and no electrodes in the water (i.e. $j=0$ and $t_{C P}=$ $0)$. In essence, the synthetic groundwater was simply stirred for $t_{M}=60 \mathrm{~min}$ and filtered, giving a measurement of the removal capacity of background ions found in Bangladesh groundwater (excluding iron) and filtration alone. 


\subsubsection{ECAR batch experiments run 2 - Matrix 2}

\section{Purpose}

A second round of batch experiments was performed using SBGW-2 synthetic groundwater (containing average levels of phosphate and silicate) and the 3 L-single chamber electrochemical cell. The purpose of this run, designated Matrix 2, was to determine the minimum dose required to reduce 500-600 $\mu \mathrm{g} / \mathrm{L}$ arsenic in water with expected levels of phosphate and silicate to below the Bangladesh and WHO limits (50

$\mu \mathrm{g} / \mathrm{L}$ and $10 \mu \mathrm{g} / \mathrm{L}$ respectively), and to expand the range of current densities observed. The single chamber electrochemical cell was used in place of the separated cathode electrochemical cell (used in Matrix 1) after internal testing revealed little to no difference in arsenic removal capacity for the two cells operating under comparable conditions.

\section{Procedural differences from Matrix 1}

For Matrix 2, a single batch experiment was run for each current density and aliquots were removed at designated times corresponding to specific charge densities. Aliquots of $50 \mathrm{~mL}$ were removed using a glass luer tip syringe from the top of the cell. Sampling times were calculated according to Equation 2.32 taking into account the new treatment volume after sampling. Current densities 1.1, 5.0, 10, 30, and $100 \mathrm{~mA} / \mathrm{cm}^{2}$ were tested with aliquots removed after dosages of 25, $5075,100,125,150$, and in some cases $175 \mathrm{C} / \mathrm{L}$. Current density $0.020 \mathrm{~mA} / \mathrm{cm}^{2}$ was sampled after dosages of 6 , $12,18,25,50$, and $75 \mathrm{C} / \mathrm{L}$ due to the higher observed removal efficiency. Each $50 \mathrm{~mL}$ aliquot was mixed for $t_{M}>60$ min before filtration using a new membrane. If As[III] 
Table 3.2: Operating parameters for Matrix 2 tests.

\begin{tabular}{lccccc}
\hline \hline $\begin{array}{l}\text { Current } \\
\text { Density } \\
\left(\mathrm{mA} / \mathrm{cm}^{2}\right)\end{array}$ & $\begin{array}{c}\text { Operating } \\
\text { Current } \\
(\mathrm{mA})\end{array}$ & $\begin{array}{c}\text { Active } \\
\text { Electrode Area } \\
\left(\mathrm{cm}^{2}\right)\end{array}$ & $\begin{array}{c}\text { Max } \\
\text { Dosage } \\
(\mathrm{C} / \mathrm{L})\end{array}$ & $\begin{array}{c}\text { Max } \\
t_{C P}{ }^{\mathrm{a}} \\
(\mathrm{min})\end{array}$ & $\begin{array}{c}\mathrm{A} / \mathrm{V}^{\mathrm{b}} \\
\left(\mathrm{cm}^{-1}\right)\end{array}$ \\
\hline & & 9 & 175 & 9.20 & 0.0030 \\
30 & 900 & 30 & 150 & 8.13 & 0.010 \\
10 & 900 & 90 & 175 & 9.20 & 0.030 \\
5.0 & 900 & 100 & 175 & 16.33 & 0.033 \\
1.1 & 500 & 100 & 175 & 74.98 & 0.033 \\
0.020 & 110 & 150 & 75 & 1199.29 & 0.050 \\
& 3 & & & & \\
\hline
\end{tabular}

a $t_{C P}$ is the total current processing time for the batch test. Note that $t_{C P}$ for lower values of charge density will be lower. Note also that the treatment volume decreases slightly as aliquots are removed, decreasing the required current processing time from that expected by Equation 2.31 .

b Unless indicated otherwise, replicated tests used the same A/V ratio.

measurements were taken, the filtrate was immediately filtered through a speciation cartridge (Section 3.2.1) and placed in a sample vial. As[III] was measured at least once for each current density and dosage. Initial arsenic concentration was measured from unfiltered aliquots removed before electrodes were placed in the cell. $\mathrm{pH}$ and $\mathrm{DO}$ were measured 2-3 minutes before electrodes were inserted as well as immediately after dosing during the mixing stage. Synthetic groundwater was made less than 24 hours prior to testing.

Different sized electrodes bent to the same shape, and made of the same iron wire, were used for these experiments in order to work within the current range of the galvonostat. Operating current, current density, and active electrode area used for each test are given in Table 3.2 . 


\section{Mixing time kinetics profile}

The mixing time for Matrix 2 tests was set at $t_{M}=60$ min based on the kinetics tests from Matrix 1 (shown in Figure 3.4). To determine if the change in water batch recipe or procedure affected the mixing kinetics profile, one kinetics profile was measured in SBGW-2 using the Matrix 2 procedure. ECAR parameters were $j=1.1 \mathrm{~mA} / \mathrm{cm}^{2}$, $q=150 \mathrm{C} / \mathrm{L}, t_{C P}=66$ min. The resulting profile is shown in Figure 3.6. Although the arsenic concentration is still decreasing at $t_{M}=60 \mathrm{~min}$, the gain in percent arsenic removal is only about $0.2 \%$ between $t_{M}=60-100 \mathrm{~min}$, or $0.005 \%$ removal per min. This indicates that slow complexation has been reached by $t_{M}=60$ min even in SBGW-2.



Figure 3.6: Mixing time kinetics profile for SBGW-2 spiked to $\mathrm{As}_{\text {tot }}=510 \mu \mathrm{g} / \mathrm{L}$ treated at $j=1.1 \mathrm{~mA} / \mathrm{cm}^{2}, q=150 \mathrm{C} / \mathrm{L}, t_{C P}=66 \mathrm{~min}$. Residual arsenic concentration (left axis) and percent arsenic removed (right axis) as a function of mixing time are shown. A dashed horizontal line marks the WHO limit $(10 \mu \mathrm{g} / \mathrm{L})$. 


\section{Replication}

All batch experiments, with the exception of $j=30 \mathrm{~mA} / \mathrm{cm}^{2}$ and $j=100$ $\mathrm{mA} / \mathrm{cm}^{2}$, were repeated and the initial and residual arsenic concentrations at each value of $j$ and $q$ were averaged. In addition, for the replicated batch tests, the maximum $q$ was increased from $150 \mathrm{C} / \mathrm{L}$ to $175 \mathrm{C} / \mathrm{L}$, and hence there is only one observation per current density at $q=175 \mathrm{C} / \mathrm{L}$. With the exception of $j=0.02 \mathrm{~mA} / \mathrm{cm}^{2}$, the repeated tests used the same $\mathrm{A} / \mathrm{V}$ ratio, and $t_{C P}$ associated with each charge density varied by $<1$ minute $^{4}$.

\section{Replicate Variability estimates}

Variations in the arsenic concentration after ECAR treatment come from the same sources in Matrix 2 experiments as in Matrix 1 (discussed in Section 3.2.6). However, procedural differences caused a decrease in the spread of measured initial arsenic concentrations compared to Matrix 1 (for Matrix 2, the average over all batch tests was $\left.A s_{i n i t}=560 \pm 30 \mu \mathrm{g} / \mathrm{L}\right)$. The expected standard deviation as a function of arsenic concentration (assuming a gaussian distribution) was estimated by fitting the replicate variability (i.e. $\sigma=\left|y_{2}-y_{1}\right| / \sqrt{2}$ for two replicates) to the average final arsenic concentration for those tests (Figure 3.7). The resulting estimated uncertainty for Matrix 2 points, $\sigma_{M 2}$, as a function of final arsenic concentration, $A s_{f}$, is (in units of $\mu \mathrm{g} / \mathrm{L}$ ):

$$
\sigma_{M 2}=0.6 \pm 1.6+(0.071 \pm 0.007) A s_{f}
$$

\footnotetext{
${ }^{4}$ Small variations in $t_{C P}$ between batch tests were due to varying the aliquot volumes between tests - see Equation 2.32 for the relationship between treatment volume (which is reduced as each aliquot is removed) and $t_{C P}$.
} 
The $R^{2}$ value for this fit was $R^{2}=0.875$. The adjusted $R^{2}$ for this fit was $R_{a d j M 2}^{2}=$ 0.867 (compared to an adjusted $R^{2}$ of $R_{a d j M 1}^{2}=-0.057$ for the Matrix 1 fit).



Figure 3.7: Deviation in the final arsenic concentration measured between replicated batch tests for Matrix 2 as a function of the average final arsenic concentration, $A s_{\text {fave }}$. The deviation plotted between two measurement $y_{1}$ and $y_{2}$ is just $\sigma=\left|y_{2}-y_{1}\right| / \sqrt{2}$. The dashed line in a linear fit weighted by $1 / A s_{\text {fave }}^{2}$.

The current processing time required to provide a given dosage is a function of the operating current $I$ and not the current density $j$ (Equation 2.31). In the Matrix 2 experiments, the current was allowed to vary between current densities, in some case leading to drastically different current processing times. To isolate the effect of current density from the effect of current processing time on arsenic removal capacity, a single batch test was performed at $1.1 \mathrm{~mA} / \mathrm{cm}^{2}$ using the same current, $I=3 \mathrm{~mA}$, and hence the same current processing time, as the earlier batch tests performed at $0.02 \mathrm{~mA} / \mathrm{cm}^{2}$. Aliquots were removed in the same manner as before at times corresponding to $q=6$, 
$12,18,25,50$, and $75 \mathrm{C} / \mathrm{L}$ (to match the samples taken during the $0.020 \mathrm{~mA} / \mathrm{cm}^{2}$ batch test). To maintain the current density, an electrode with $2.73 \mathrm{~cm}^{2}$ active area was used $\left(\mathrm{A} / \mathrm{V}=0.00091 \mathrm{~cm}^{-1}\right)$.

\subsubsection{Adsorption using post-synthesis ECAR-generated adsorbent}

Two batch experiments, PSA 1 and PSA 2, were performed to measure the arsenic removal capacity of ECAR-generated iron (hydr)oxides particles allowed to form and age before being exposed to arsenic. To obtain post-synthesis ECAR-generated (PSEGA) adsorbent, synthetic groundwater was prepared according to the SBGW-2 recipe (with no initial arsenic) and dosed using the 3 L-single chamber electrochemical cell at $j$ $=5.0 \mathrm{~mA} / \mathrm{cm}^{2}, q=175 \mathrm{C} / \mathrm{L}$, and $t_{C P}=17.3 \mathrm{~min}$. After dosing, the solution was divided into Sample A (2 liters) and Sample B (1 liter). In sample A, arsenic was added at the start of mixing, and in Sample B, arsenic was added after 60 minutes of aging (while stirred). In both cases, the solution was mixed an additional time $t_{M}$ after arsenic was added, followed by filtration (note that $t_{M}$ does not include the 60 minutes of stirring prior to arsenic addition for Sample B).

Arsenate and arsenite stock solutions were added sequentially to sample A and sample B solutions (while stirring) in an amount corresponding to a final solution concentration of $300 \mu \mathrm{g} / \mathrm{L} \mathrm{As}[\mathrm{V}]$ plus $300 \mu \mathrm{g} / \mathrm{LAs}[\mathrm{III}]$ (ratios of stock As[V] and As[III] solution added to existing solution were $6 \cdot 10^{-4}$ and $6 \cdot 10^{-3}$ respectively). Stock solutions were the same as those used to make water batches (see Section 3.2.3). During the first batch experiment, unfiltered aliquots were removed to measure initial arsenic within 
30 seconds of adding arsenic $\left(\mathrm{HNO}_{3}\right.$ preservative was added right away to prevent precipitation and settling in the sample vial). However, the measured concentrations were quite low compared to expected values $\left(\mathrm{As}_{t o t}=210\right.$ and $300 \mu \mathrm{g} / \mathrm{L}$ for samples $\mathrm{A}$ and B respectively), suggesting that the arsenic had not fully mixed within the solution at the time of sampling. During the second batch experiment, aliquots to measure initial arsenic were removed 60 minutes after arsenic was added to solution (again, the aliquots were not filtered and preservative was added right away to prevent settling in the sample vial). These results were much closer to the expected amount of arsenic $\left(\mathrm{As}_{t o t}=630\right.$ and $590 \mu \mathrm{g} / \mathrm{L}$ for $\mathrm{A}$ and $\mathrm{B}$ respectively). Therefore, the initial arsenic level for both samples A and B in Exp 1 were estimated to be the average of measured initial arsenic for samples A and B in Exp $2\left(\mathrm{As}_{t o t}=610 \mu \mathrm{g} / \mathrm{L}\right)$.

In Exp 1, aliquots were removed from the top (using a glass luer tip syringe) of both samples A and B after $t_{M}=20,40,60,80,100,120$ min. Aliquots were $40 \mathrm{~mL}$ for all but $t_{M}=60 \mathrm{~min}$, when an aliquot of $\approx 500 \mathrm{~mL}$ was removed for XAS sample preparation (Section 4.2.4). In Exp 2, only one aliquot of $\approx 500 \mathrm{~mL}$ was removed after $t_{M}=60$ min. In both Exp 1 and 2 , a mixing time $t_{M}=60$ min was used to compare to Matrix 2 coprecipitation tests. After mixing for time $t_{M}=120 \mathrm{~min}$ and $t_{M}=60$ min for Exp 1 and 2 respectively, unfiltered samples of each solution (A and B) were placed in beakers and allowed to settle overnight. The samples were filtered and tested after settling for 1 and 2 days (1 day only for Exp 2). This was done to determine if further complexation and coagulation was still occurring. These points cannot properly be taken as an extension $t_{M}$ since the solution was not being mixed. 


\subsubsection{Polarization studies}

Voltammetry is a sensitive technique used to probe the electrochemical behavior of a system. It involves adjusting the potential of an electrochemical cell and observing the resulting current response or polarization curve (i.e an $i$-E curve, discussed in Section 2.5. $1^{5}$ ). The magnitude of the current flowing through the cell will be determined by the rates of heterogeneous and homogeneous reactions occurring in each half-cell as well as the rate at which reactants in solution can be transported to and from the electrodes by diffusion, migration, or convection. If the potential is scanned at a predetermined rate, analysis of the current response as a function of time can lead to useful information about the different reactions occurring in a cell as well as the relative reaction rates. If a three-electrode cell (discussed in Section 2.5.1) is used, then the working electrode can be isolated and the current response will reflect reaction rates and mass transport occurring at the working electrode-solution interface only.

Voltammetry is also extremely useful to determine if passivation is occurring on an electrode. Passivation can occur when an oxide layer forms on the electrode surface, preventing further electron transfer. This can appear very prominently on an i-E curve, as the net current will decrease to zero when passivation is complete.

Polarization curves were measured for the iron wire electrode used in Matrix 1 and Matrix 2 experiments using a $60 \mathrm{~mL}$ three-electrode cell. The three-electrode cell was fabricated as depicted in Figure 3.8 including a platinum counter electrode (surface area $\left.1.07 \mathrm{~cm}^{2}\right)$ and an $\mathrm{Ag} / \mathrm{AgCl}(3 \mathrm{M} \mathrm{NaCl})$ single junction reference electrode $(5.7 \mathrm{~mm}$

\footnotetext{
${ }^{5}$ This text relies heavily on background presented in Section 2.5.1, and it will be assumed here that the reader is familiar with its contents
} 
outer diameter) with a vycor frit (Bioanalytical). The working electrode was fashioned from iron wire by covering the length of the wire in epoxy resin and filing one end to expose a disk of iron with a diameter of $0.18 \mathrm{~cm}$ (surface area $0.025 \mathrm{~cm}^{2}$ ). The exposed end was bent to be within $1 \mathrm{~mm}$ of the reference electrode tip to reduce the IR potential drop (uncompensated resistance) due to the electrolyte (see Figure 3.8). Solution was purged with $\mathrm{N}_{2}$ gas for approximately 2 min before conditioning for each scan and $\mathrm{N}_{2}$ gas was used to purge the headspace during conditioning and scanning. The dissolved oxygen levels at the end of any given scan were $<0.10 \mathrm{mg} / \mathrm{L}$. The entire apparatus was placed inside a grounded copper mesh faraday cage to reduce electrical interference (primarily needed for EIS experiments, as explained in Section 3.2.10). Scans were performed with a Gamry Instruments Femptostat CE scanning potentiostat running Gamry Framework software v4.35.

The $\mathrm{Ag} / \mathrm{AgCl}$ reference electrode was tested against a second saturated calomel reference electrode (Beckman; ceramic frit) before use. The vycor frit on the reference electrode was secured to the glass electrode body using heat shrink wrap that was relatively easy to loosen, potentially allowing ions to bypass the vycor frit. To see if this was the case, the electrical impedance of the reference electrode was measured with only the vycor frit submerged and compared to the impedance with the potentially lose shrink wrap seal submerged. The impedance did not change, and so it was assumed that ions were not able to bipass the vycor frit through the shrink wrap seal.

The working electrode was sanded using Silcon Carbide 1500 mirror fine waterproof sandpaper before each scan. It was later suggested that non-reproducible back- 




Figure 3.8: Schematic of Three-Electrode Cell used for polarization studies and Electronic Impedance Spectroscopy (EIS). The cell contained a platinum counter electrode, an $\mathrm{Ag} / \mathrm{AgCl}$ reference electrode, and an exposed iron disk working electrode (surface area $0.025 \mathrm{~cm}^{2}$ ). The cell is described more fully in the text (Section 3.2.9). 
ground currents could be reduced if the electrode surface was systematically polished before each test using alumina or diamond paste as the polishing material. Background currents did not appear to be an issue for this round of tests, but more rigorous polishing should be adhered to in the future.

Polarization curves for the iron wire electrode were measured at a scan rate of $\nu=0.1 \mathrm{mV} / \mathrm{s}$ in $0.1 \mathrm{M} \mathrm{KClO}_{4}$ (Alfa Aesar) added to DI water and $0.1 \mathrm{M} \mathrm{KClO}_{4}$ added to synthetic Bangladesh groundwater (SBGW-2 recipe, see Table 3.1). The perchlorate salt was added to reduce the IR-drop between the reference and working electrode ${ }^{6}$. Perchlorate was chosen because it dissociates substantially at neutral $\mathrm{pH}$, the $\mathrm{ClO}_{4}^{-}$ions are slow to oxidize at room temperature in the voltage range scanned, and the ions do not form complexes with metal. Before each scan, the iron electrode was conditioned at a constant current of - 1nA for 6 hours. Open circuit voltage for plain perchlorate and perchlorate plus SBGW-2 was $-0.570 \mathrm{~V}$ and $-0.630 \mathrm{~V}$ vs $\mathrm{Ag} / \mathrm{AgCl}$ respectively. Scans started at the open circuit voltage scanning up to $-0.1 \mathrm{~V}$, down to $-1.6 \mathrm{~V}$ and back up to $-0.1 \mathrm{~V}$. Oxidation curves were derived from the second pass between open circuit voltage and $-0.1 \mathrm{~V}$. This ensured that any passive layer which might have formed on the iron electrode while exposed to air was completely reduced before the polarization curve.

The $\mathrm{pH}$ of the solution were measured before the initial $\mathrm{N}_{2}$ purge as well as after the completion of the scan. For plain perchlorate solution, the initial $\mathrm{pH}$ was $p H_{i}$ $=6.80$ and the final value, $p H_{f}=9.60$. For perchlorate plus SBGW, the values were

\footnotetext{
${ }^{6} \mathrm{DC}$ transient voltammetry requires concentrations above $0.1 \mathrm{mM}$ in order to achieve a significant Faradic current signal relative to background. Trace concentrations down to $10^{-7} \mathrm{M}$ may be determined using AC, pulse, or square-wave methods to enhance the Faradic-to-background current ratio (Bond, 2002).
} 
$p H_{i}=7.62$ and $p H_{f}=9.14$. The majority of this change likely occurred during the initial $\mathrm{N}_{2}$ purge and before the start of the scan, however, there is no way to be sure ${ }^{7}$.

\subsubsection{Electrochemical Impedance Spectroscopy}

Interpreting the results of Electrochemical Impedance Spectroscopy scans is based on the assumption that an equivalent circuit can be defined for an electrochemical system and the basic form of the equivalent circuit can be determined from the system's impedance response when driven at different frequencies. The impedance, $Z$, is measured by exciting the system with a potential $E(t)=E_{0} \cos (\omega t)$ and measuring the response current $I(t)=I_{0} \cos (\omega t-\phi)$. The current magnitude and phase shift measured in the response give the complex impedance at frequency $\omega$ according to:

$$
Z(\omega)=\frac{E}{I}=Z_{0}(\cos (\phi)+i \sin (\phi))
$$

If $Z$ is measured for a series of different excitation frequencies, the real and imaginary parts can be plotted on a Nyquist plot such as that shown in Figure 3.9. Note that the $\mathrm{Y}$-axis is negative and that each point on the Nyquist plot is the impedance at one frequency, $\omega$. The Nyquist plot shown is for a simple equivalent circuit with a single time constant, showing a characteristic semi-circle. EIS plots are often made up of numerous time constants, and often only a portion of the semi-circle created by a single time constant is measured. For a given electrochemical system, the measured Nyquist plot is compared or fit to the expected Nyquist plot that would result from an equivalent

\footnotetext{
${ }^{7} \mathrm{PH}$ could not be measured immediately after the initial $\mathrm{N}_{2}$ purge because this would allow oxygen back into the chamber.
} 
circuit. Knowledge of the reaction pathways in the real system in inferred from matching the behavior of different pathways to the behavior of equivalent circuit elements.

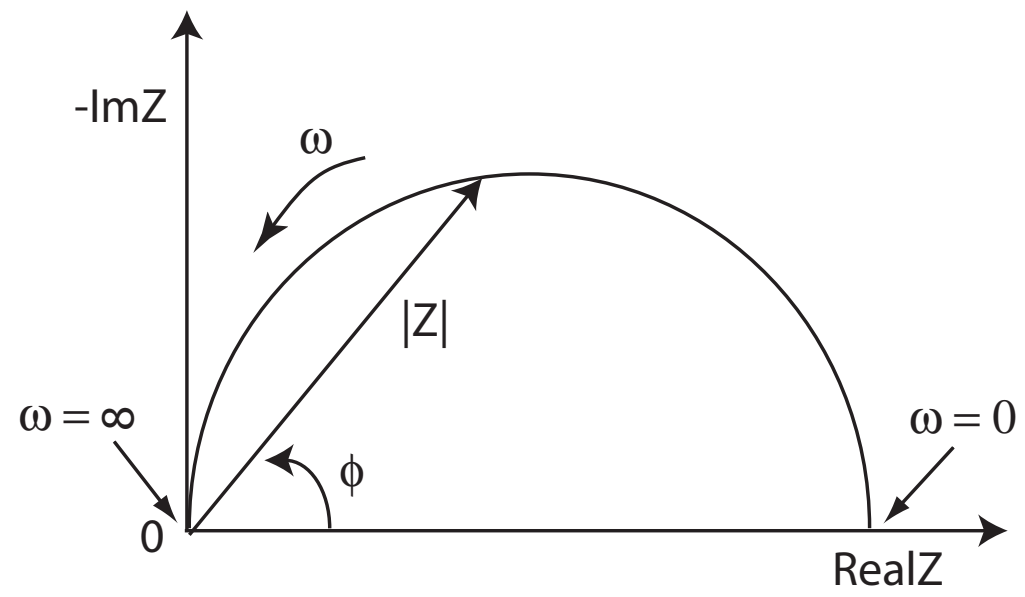

Figure 3.9: A simple Nyquist plot showing complex impedance vector $Z(\omega)$. Note that the $\mathrm{Y}$-axis is negative and that each point on the Nyquist plot is the impedance at one frequency, $\omega$. In this plot, low frequencies appear on the right side and high frequencies appear on the left. This is the case when the impedance falls as the frequency rises (usually, but not always, true).

Equivalent circuit elements must have a basis in electrochemical theory to be useful. Some of the common elements found in a three-electrode electrochemical cell include basic resistance and capacitance. Resistances include electrolyte resistance between the working and reference electrode, polarization resistance as the potential is changed which includes the kinetics of reactions and rates of diffusion to and from the electrode, and charge-transfer resistance based on single kinetically controlled reactions in equilibrium. Capacitance is caused by the electrical charge that gathers at the electrode surface to compensate for charge in the surrounding electrolyte (forming what is called the double layer capacitance). Diffusion can lead to impedance as well since, at high frequencies, the ions don't have to move very far to respond, whereas at low 
frequencies, they must diffuse farther. Impedance due to diffusion is known as Warburg impedance. Other more complicated electrochemical cell behavior can be modeled using more complicated circuit elements as well, making EIS a powerful technique for understanding cell behavior.

In practice, this technique suffers from some important limitations. For example, impedance analysis is much easier in linear systems (circuits) than non-linear ones. However, electrochemical systems are highly non-linear (doubling the voltage in general will not double the current). Thus in normal EIS practice, only a small (1 to $10 \mathrm{mV})$ AC signal is applied to confine the measurement to a pseudo-linear segment of the cell's current versus voltage curve. A second limitation is that the system must be in steady state over many hours to take an EIS spectrum. In practice, this is very difficult to achieve and may lead to very inaccurate results. Finally, this technique is also highly dependent on the equivalent circuit model chosen by the researcher. Numerous models may fit the same impedance response, and additional knowledge of the system is required to chose the most appropriate one.

EIS scans were performed to measure the electrolyte resistance in synthetic Bangladesh groundwater (SBGW-1). The same three-electrode cell shown in Figure 3.8 was used (described in the text of Section 3.2.9) hooked up to a Gamry Instruments Femptostat CE scanning potentiostat running Gamry Framework software EIS300 v.4.35. Before each scan, the current was monitored until approximate steady state was reached (usually 40 - 120 seconds). AC potential with an amplitude of $10 \mathrm{mV}$ was applied around the open circuit voltage $(-0.65 \mathrm{~V})$ in a frequency range of $1-100,000 \mathrm{~Hz}$ (10 data points 
per decade). It was found through trial and error that the results were much less noisy when the three-electrode cell was surrounded by a grounded copper mesh Faraday cage, so the cage was used for all measurements. The solution was $68 \mathrm{~mL}$ of freshly prepared synthetic Bangladesh groundwater (SBGW-1 recipe) with initial $\mathrm{pH}=7.00$ and $\mathrm{DO}=$ $1.53 \mathrm{mg} / \mathrm{L}$ (solution was purged with $\mathrm{N}_{2}$ ).

The setup and theory was tested using a dummy cell, which is just a real circuit containing the components shown in Figure 3.11. The dummy circuit values were $R_{e}=200 \Omega$ and $R_{c t}=3000 \Omega$. The EIS scan showed a nice semi-circle shape with low frequency intercept at $Z_{\text {real }}\left(\omega_{\text {low }}\right)=3210 \pm 20 \Omega$ and a high frequency intercept at $Z_{\text {real }}\left(\omega_{\text {high }}\right)=201 \pm 3 \Omega$, correctly recovering the values of $R_{c t}+R_{e}$ and $R_{e}$ respectively, indicating that the setup was sound and correctly calibrated to within $2 \%$.

\subsubsection{Sedimentation tests}

Sedimentation was compared to $0.1 \mu \mathrm{m}$ vacuum filtration as a means to separate ECAR generated particles from potable water during both the Matrix 1 and Matrix 2 batch experiments. During Matrix 1, an additional batch test was performed for each of four experiments with different operating conditions (including the following: (1) $j$ $=1.1 \mathrm{~mA} / \mathrm{cm}^{2}, q=23 \mathrm{C} / \mathrm{L}, t_{C P}=3 \mathrm{~min} ;(2) j=1.1 \mathrm{~mA} / \mathrm{cm}^{2}, q=85 \mathrm{C} / \mathrm{L}, t_{C P}=5$ $\min ;(3) j=0.07 \mathrm{~mA} / \mathrm{cm}^{2}, q=25 \mathrm{C} / \mathrm{L}, t_{C P}=50 \mathrm{~min}$; and (4) $j=0.70 \mathrm{~mA} / \mathrm{cm}^{2}, q$ $\left.=15 \mathrm{C} / \mathrm{L}, t_{C P}=3 \mathrm{~min}\right)$. Dosed water from each batch test $(850 \mathrm{~mL})$ was placed in a 1-liter beaker and allowed to sit quiescently ${ }^{8}$ (no additional mixing was performed). At

${ }^{8}$ For this work, quiescent refers to sitting without mixing or shaking of any kind. 
designated times, small aliquots were removed from the top of the beaker using a luer tip syringe and tested for arsenic.

A brief test was conducted to determine if magnets could accelerate the separation of ECAR- generated particles from potable water. The mixed effluent from two Matrix 1 tests was placed in two small beakers - one with powerful flat magnets (salvaged from computer hard drives) taped to the bottom and one with no magnets. The two beakers were allowed to sit and visually evaluated one day later. Aliquots from the top of the beaker were testing using the QuickTest, but these values were not verified by ICPMS.

During Matrix 2 experiments, SBGW-2 water (Matrix 2 procedure) was used that had been dosed at $j=1.1 \mathrm{~mA} / \mathrm{cm}^{2}, q=100 \mathrm{C} / \mathrm{L}$, and $t_{C P}=44.7 \mathrm{~min}$. One portion of the dosed water was mixed for 30 minutes using a magnetic stirrer and set aside, while the rest was mixed for 60 minutes, providing two samples for experimentation to see if prior mixing could accelerate the settling process. After mixing, samples sat quiescent and uncovered in 1-liter glass beakers to allow settling. In each sample, aliquots were removed from the top $2 \mathrm{~cm}$ using glass luer tip syringe at intervals from 0 to 45 hours after mixing ceased. Half of the aliquot was filtered using a $0.1 \mu \mathrm{m}$ membrane before arsenic testing and half the sample was not filtered. 


\subsection{Results and Discussion}

\subsubsection{Resistivity of synthetic Bangladesh water}

Electrochemical Impedance Spectroscopy (EIS) was used to measure the resistivity of synthetic Bangladesh groundwater (SBGW-1 recipe). Several EIS scans were performed about the open circuit potential in freshly prepared SBGW-1 water (full procedure described in Section 3.2.10). The high frequency portion (near $100 \mathrm{kHz}$ ) of a representative scan (Figure 3.10) reveals half of the semi-circle shape characteristic of a single time constant (similar to the example shown in Figure 3.9). A simple equivalent circuit with a single time constant was used to model the system. This circuit, shown schematically in Figure 3.11, includes a double-layer capacitance, $C_{d l}$, in parallel with resistance due to charge-transfer and polarization, $R_{c t}$, which occurs across the double layer (the electrochemical meaning of these elements is explained more fully in Section 3.2.10). $C_{d l}$ and $R_{c t}$ are added in series to the resistance of the electrolyte, $R_{e}$ between the working and reference electrode.

An EIS scan of the circuit shown in Figure 3.11 would produce a semi-circle on a Nyquist plot crossing the real axis at two points. At very high frequencies, the capacitor will contribute very little to the impedance $Z$, and hence most of the current will flow through the capacitor $C_{d l}$ and resistor $R_{e}$, bypassing the resistor $R_{c t}$. Thus the high frequency real axis intercept will correspond to the resistance ${ }^{9} Z_{\text {real }}\left(\omega_{\text {high }}\right)=$ $R_{e}$. Using the circuit in Figure 3.11 as a model, the bulk resistance of the electrolyte

\footnotetext{
${ }^{9}$ Conversely, at low frequencies, the impedance of the capacitor is very high and most current will flow through the resistors $R_{c t}$ and $R_{e}$ in series. The real value of $Z$ at this point should be $Z_{\text {real }}\left(\omega_{l o w}\right)=$ $R_{c t}+R_{e}$, however, this does not concern the current measurement.
} 


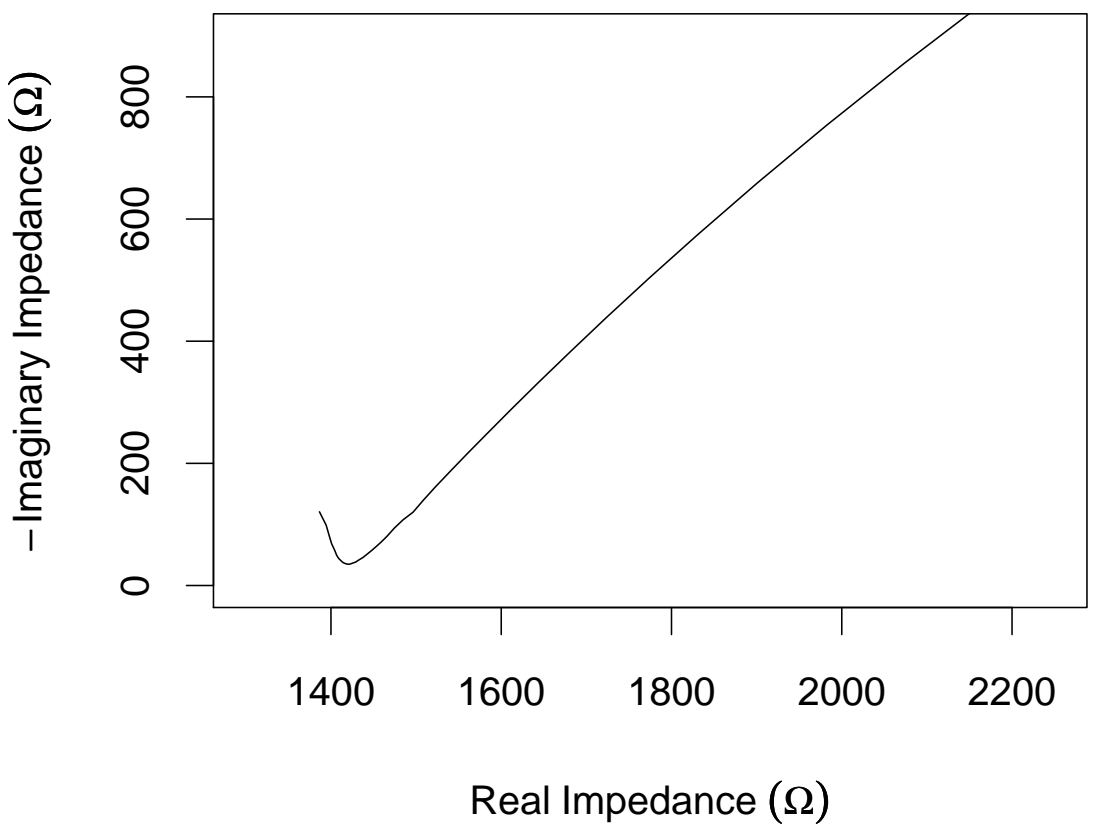

Figure 3.10: A Nyquist plot for a representative electrochemical impedance scan about the open circuit potential in synthetic Bangladesh groundwater (SBGW-1 recipe).

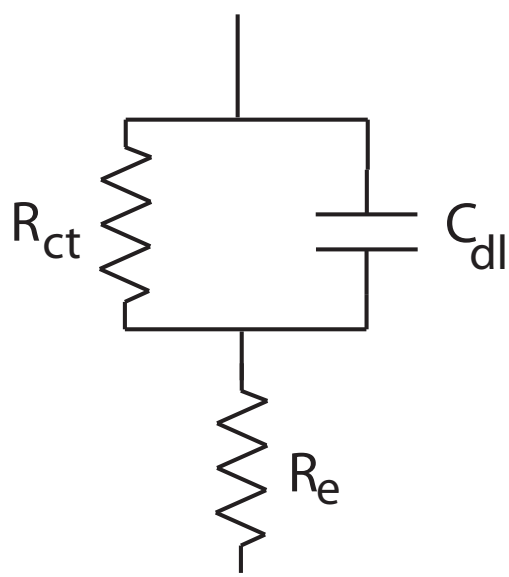

Figure 3.11: Equivalent circuit used in EIS analysis to measure electrolyte resistance of synthetic Bangladesh groundwater. Components include the double-layer capacitance, $C_{d l}$, the charge-transfer resistance, $R_{c t}$, and the electrolyte resistance, $R_{e}$. 
should correspond to the high frequency impedance real axis intercept measured from Figure 3.10.

The high frequency impedance real axis intercept averaged over 4 separate EIS scans is $1420 \pm 180 \Omega$. This corresponds to an electrolyte resistance of $R_{e}=1420 \pm 180 \Omega$. The resistance is measured across a gap of $1 \mathrm{~mm}$ over an electrode area of $0.025 \mathrm{~cm}^{2}$, leading to a resistivity of $\rho_{e}=355 \Omega \cdot \mathrm{cm}$. As expected from the composition of SBGW-1, the resistivity is very high. However, it is still not as high as the $1000-2000 \Omega \cdot \mathrm{cm}$ reported for groundwater in Bangladesh (Burgess \& Ahmed (2006), converted from a conductivity of $500-1000 \mu \mathrm{S} / \mathrm{cm})$. This could partially be due to the excess sulfate in SBGW-1 compared to real Bangladesh groundwater (sulfate was reduced from 81 to $8 \mathrm{mg} / \mathrm{L}$ in SBGW-2 to be more consistent with real values). In either case, the resistivity is high enough to suggest that the voltage required of constant current treatment may be high in Bangladesh if no modifications are made. A small amount of table salt (below the taste threshold) added to the water before treatment could potentially increase the conductivity and lower the power consumption of treatment - this should be explored in future research.

It is important to note that only a small fraction of the power of EIS has been employed in this measurement. EIS could be very useful to determine the reactions occurring during ECAR treatment. For example, EIS scans around different mean potentials would likely be able to distinguish whether the iron electrode in ECAR is producing $\mathrm{Fe}^{2+}$ leading to Fe[II]hydr(oxides) that oxidize to Fe[III]hydr(oxides), or $\mathrm{Fe}^{3+}$ leading to Fe[III]hydr(oxides) directly (the former would be associated with additional resistance). 
This was the original intention for applying it to this work, however time constraints did not allow EIS to be fully utilized. This single measurement and detailed methods section (Section 3.2.10) can act as a guide for the student or students who carry on and expand on this work. This measurement does verify a working setup and capability to continue EIS scans in the future. Suggested future measurements include (1) EIS scans around a series of potentials associated with the current densities used in Matrix 1 and Matrix 2 to determine if a different chemical pathway (characterized by a different impedance) predominates at different current densities, and (2) a bulk resistance measurement of the SBGW-2 synthetic and real groundwater in ECAR prototype units.

\subsubsection{Arsenic removal capability in synthetic Bangladesh water}

It is important that any technology suitable for Bangladesh be able to reduce relevant arsenic levels below either the legal maximum in Bangladesh $(50 \mu \mathrm{g} / \mathrm{L})$, or the more conservative maximum recommended limit by WHO $(10 \mu \mathrm{g} / \mathrm{L})$ in Bangladesh groundwater. Batch tests of ECAR treatment have been performed over a range of charge and current densities ( $q$ and $j$ ) using two recipes for synthetic Bangladesh groundwater - SBGW-1 and SBGW-2 (composition described in Table 3.1). SBGW-1 and SBGW-2 differed in the amount of phosphate and silicate added; SBGW-1 had very low levels of phosphate and silicate while SBGW-2 had average levels of both ions relative to tube wells in Bangladesh. In each case, SBGW was spiked with $300 \mu \mathrm{g} / \mathrm{L} \mathrm{As}[\mathrm{III}]$ and $300 \mu \mathrm{g} / \mathrm{L}$ $\mathrm{As}[\mathrm{V}]\left(\mathrm{As}_{t o t}=600 \mu \mathrm{g} / \mathrm{L}\right)$.

Residual arsenic concentrations after batch tests in SBGW-1 are listed in Ta- 
Table 3.3: Results of ECAR batch tests in synthetic Bangladesh groundwater (SBGW1) containing low phosphate and no silicate. Initial and residual arsenic concentrations are averaged over replicate batch tests. Boldface indicates the lowest residual arsenic concentration achieved for each current density. Errors on arsenic measurements are $10 \%$.

\begin{tabular}{|c|c|c|c|c|c|c|}
\hline $\begin{array}{c}\text { Current } \\
\text { Density } \\
\left(\mathrm{mA} / \mathrm{cm}^{2}\right)\end{array}$ & $\begin{array}{c}\text { Charge } \\
\text { Density } \\
(\mathrm{C} / \mathrm{L})\end{array}$ & $\begin{array}{c}\text { Current } \\
\text { Processing Time } \\
\text { (min) }\end{array}$ & $\begin{array}{c}\text { Initial } \\
\text { As } \\
(\mu \mathrm{g} / \mathrm{L})\end{array}$ & $\begin{array}{l}\text { Residual } \\
\text { As } \\
(\mu \mathrm{g} / \mathrm{L})\end{array}$ & $\begin{array}{c}\% \text { As } \\
\text { Removed }\end{array}$ & $\begin{array}{l}\text { Num } \\
\text { Replicates }\end{array}$ \\
\hline $0^{\mathrm{a}}$ & $0^{\mathrm{a}}$ & 0 & 580 & 530 & 8 & 2 \\
\hline $0^{\mathrm{b}}$ & $0^{\mathrm{b}}$ & $11^{\mathrm{c}}$ & 580 & 200 & 65 & 2 \\
\hline $\mathbf{0}^{\mathrm{b}}$ & $\mathbf{0}^{\mathrm{b}}$ & $\mathbf{5 0}^{\mathrm{c}}$ & 580 & 93 & 84 & 2 \\
\hline 0.07 & 1.5 & 3 & 560 & 370 & 34 & 1 \\
\hline 0.07 & 2.5 & 5 & 590 & 220 & 64 & 2 \\
\hline 0.07 & 5.4 & 11 & 600 & 160 & 73 & 1 \\
\hline 0.07 & 25 & 50 & 620 & 19 & 97 & 2 \\
\hline 0.30 & 6.4 & 3 & 580 & 170 & 71 & 1 \\
\hline 0.30 & 11 & 5 & 590 & 260 & 57 & 2 \\
\hline 0.30 & 23 & 11 & 580 & 130 & 78 & 1 \\
\hline 0.30 & 110 & 50 & 560 & 6.4 & 99 & 2 \\
\hline 0.70 & 15 & 3 & 600 & 160 & 74 & 2 \\
\hline 0.70 & 25 & 5 & 590 & 84 & 86 & 2 \\
\hline 0.70 & 54 & 11 & 530 & 29 & 95 & 1 \\
\hline 0.70 & 250 & 50 & 580 & 5.7 & 99 & 1 \\
\hline 1.1 & 23 & 3 & 580 & 60 & 90 & 3 \\
\hline 1.1 & 39 & 5 & 530 & 54 & 90 & 1 \\
\hline 1.1 & 85 & 11 & 620 & 8.5 & 99 & 2 \\
\hline 1.1 & 390 & 50 & 580 & 4.3 & 99 & 1 \\
\hline
\end{tabular}

a In this case, electrodes were never in contact with the solution, though the solution was stirred for $t_{M}=$ $60 \mathrm{~min}$ and filtered, similar to other batch tests.

b For these tests no external voltage was applied, but the electrodes were in contact and some small current $\left(0.0004-0.007 \mathrm{~mA} / \mathrm{cm}^{2}\right)$ flowed through the electrodes due to natural electrochemical processes.

c In this case, current processing time represents the amount of time that the electrodes were in contact with solution, though no external voltage was applied. 

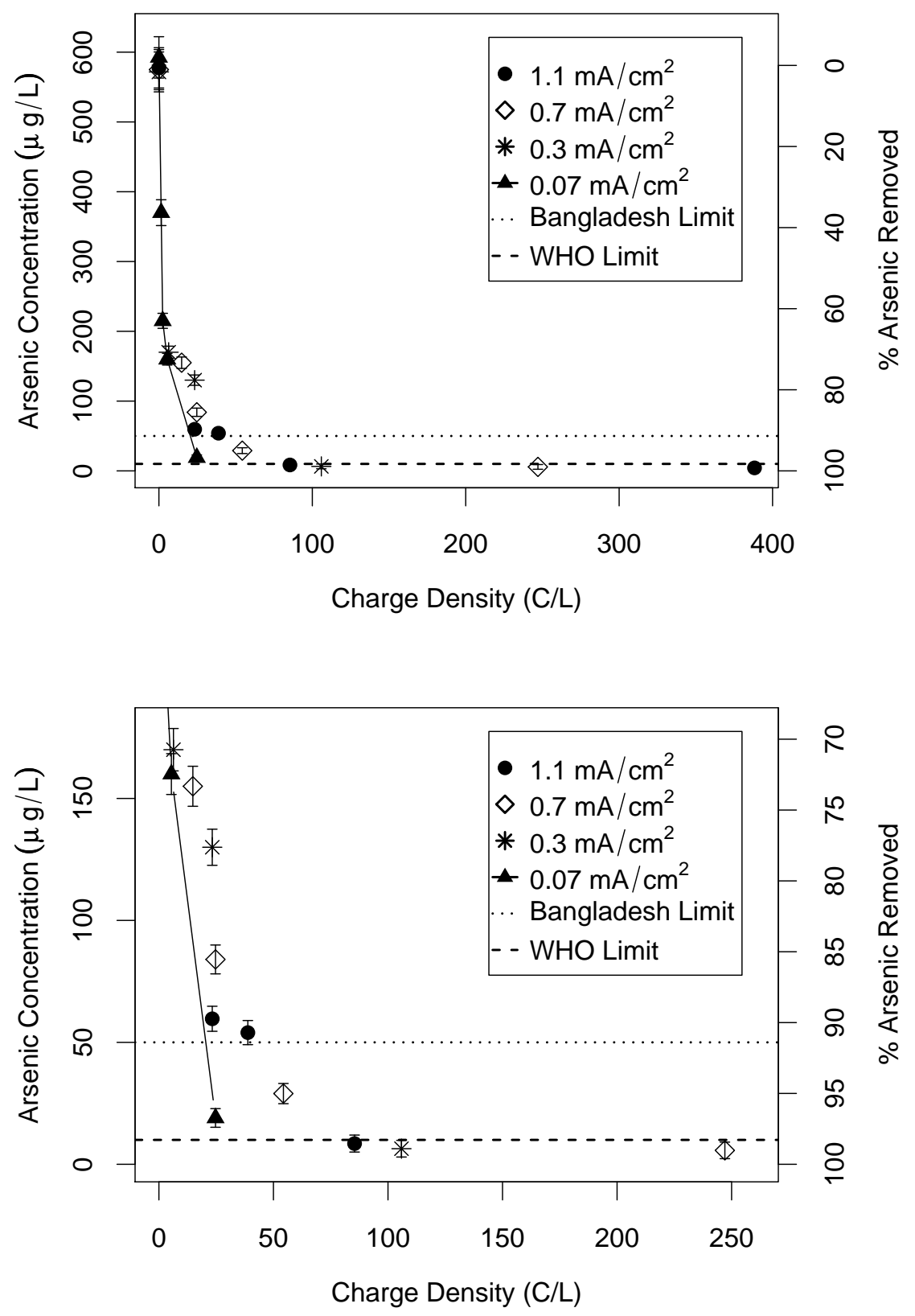

Figure 3.12: Arsenic removal (concentration on left vertical axis; percent arsenic removed on right vertical axis) as a function of charge density, $q$, after ECAR treatment at current densities $0.07-1.1 \mathrm{~mA} / \mathrm{cm}^{2}$ of low phosphate, zero silicate synthetic Bangladesh groundwater (SBGW-1 recipe - Matrix 1 conditions). Lower plot presents same data as upper plot on a reduced vertical scale to show detail at low arsenic concentrations. Percent arsenic removed is based on the average initial arsenic concentration. The WHO and Bangladesh maximum allowable arsenic limits are shown as dashed and dotted lines respectively. Error bars represent the variability between identical batch tests. 
Table 3.4: Results of ECAR batch tests in synthetic Bangladesh groundwater SBGW2, including the average concentrations of phosphate and silicate found in Bangladesh. Initial and residual arsenic concentrations are averaged over replicate batch tests. Boldface indicates the lowest residual arsenic concentration acheived for each current density. Errors on arsenic measurements are $10 \%$.

\begin{tabular}{|c|c|c|c|c|c|c|}
\hline $\begin{array}{c}\text { Current } \\
\text { Density } \\
\left(\mathrm{mA} / \mathrm{cm}^{2}\right)\end{array}$ & $\begin{array}{c}\text { Charge } \\
\text { Density } \\
(\mathrm{C} / \mathrm{L})\end{array}$ & $\begin{array}{c}\text { Current } \\
\text { Processing Time } \\
\text { (min) }\end{array}$ & $\begin{array}{c}\text { Initial } \\
\mathrm{As} \\
(\mu \mathrm{g} / \mathrm{L})\end{array}$ & $\begin{array}{c}\text { Residual } \\
\text { As } \\
(\mu \mathrm{g} / \mathrm{L})\end{array}$ & $\begin{array}{c}\% \text { As } \\
\text { Removed }\end{array}$ & $\begin{array}{c}\text { Num } \\
\text { Replicates }\end{array}$ \\
\hline 0.02 & 6 & 97.5 & 580 & 260 & 56 & 1 \\
\hline 0.02 & 12 & 195.5 & 580 & 58 & 90 & 1 \\
\hline 0.02 & 18 & 291 & 580 & 16 & 97 & 1 \\
\hline 0.02 & 25 & 407.27 & 560 & 9.7 & 98 & 2 \\
\hline 0.02 & 50 & 805.54 & 560 & 4.9 & 99 & 2 \\
\hline 0.02 & 75 & 1199.29 & 560 & 6 & 99 & 2 \\
\hline 0.02 & 100 & 1638.88 & 540 & 4.2 & 99 & 1 \\
\hline 0.02 & 125 & 2041.67 & 540 & 2.8 & 99.5 & 1 \\
\hline 0.02 & 150 & 2441.67 & 540 & 1.8 & 99.7 & 1 \\
\hline 1.1 & 25 & 11.24 & 550 & 130 & 77 & 2 \\
\hline 1.1 & 50 & 22.37 & 550 & 57 & 90 & 2 \\
\hline 1.1 & 75 & 33.38 & 550 & 33 & 94 & 2 \\
\hline 1.1 & 100 & 44.27 & 550 & 20 & 96 & 2 \\
\hline 1.1 & 125 & 55.03 & 550 & 14 & 97 & 2 \\
\hline 1.1 & 150 & 65.67 & 550 & 8.2 & 98 & 2 \\
\hline 1.1 & 175 & 74.98 & 590 & 6.0 & 99 & 1 \\
\hline 5 & 25 & 2.48 & 520 & 150 & 71 & 2 \\
\hline 5 & 50 & 4.92 & 520 & 75 & 86 & 2 \\
\hline 5 & 75 & 7.33 & 520 & 46 & 91 & 2 \\
\hline 5 & 100 & 9.7 & 520 & 33 & 94 & 2 \\
\hline 5 & 125 & 12.07 & 520 & 23 & 96 & 2 \\
\hline 5 & 150 & 14.39 & 520 & 14 & 97 & 2 \\
\hline 5 & 175 & 16.33 & 540 & 10 & 98 & 1 \\
\hline 10 & 25 & 1.38 & 560 & 160 & 72 & 2 \\
\hline 10 & 50 & 2.72 & 580 & 72 & 88 & 1 \\
\hline 10 & 75 & 4.08 & 560 & 55 & 90 & 2 \\
\hline 10 & 100 & 5.42 & 560 & 36 & 93 & 2 \\
\hline 10 & 125 & 6.73 & 560 & 27 & 95 & 2 \\
\hline 10 & 150 & 8.04 & 560 & 18 & 97 & 2 \\
\hline 10 & 175 & 9.2 & 580 & 11 & 98 & 1 \\
\hline 30 & 25 & 1.38 & 530 & 160 & 71 & 1 \\
\hline 30 & 50 & 2.75 & 530 & 80 & 85 & 1 \\
\hline 30 & 75 & 4.12 & 530 & 47 & 91 & 1 \\
\hline 30 & 100 & 5.47 & 530 & 33 & 94 & 1 \\
\hline 30 & 125 & 6.8 & 530 & 24 & 95 & 1 \\
\hline 30 & 150 & 8.13 & 530 & 14 & 97 & 1 \\
\hline 100 & 25 & 1.37 & 570 & 170 & 71 & 1 \\
\hline 100 & 50 & 2.72 & 570 & 84 & 85 & 1 \\
\hline 100 & 75 & 4.05 & 570 & 57 & 90 & 1 \\
\hline 100 & 100 & 5.37 & 570 & 40 & 93 & 1 \\
\hline 100 & 125 & 6.67 & 570 & 30 & 95 & 1 \\
\hline 100 & 150 & 7.95 & 570 & 12 & 98 & 1 \\
\hline 100 & 175 & 9.2 & 570 & 13 & 98 & 1 \\
\hline
\end{tabular}

a As explained in Section 3.2.7, the current processing time differs slightly from that expected by Equation 2.34 or 2.31 due to small solution volume changes as aliquots were removed for testing (changing A/V slightly). The current processing time associated with each charge density was instead calculated using Equation 2.32 and the solution volume as a function of time (based on the aliquote removal schedule). Replicate experiments sometimes followed slightly different aliquot removal schedules, leading to slightly different $(<4 \%)$ current processing times between tests. The average current processing time is listed for each test. 



Figure 3.13: Arsenic removal (concentration on left vertical axis; percent arsenic removed on right vertical axis) as a function of charge density, $q$, after ECAR treatment at current densities $0.02-100 \mathrm{~mA} / \mathrm{cm}^{2}$ of average phosphate, average silicate synthetic Bangladesh groundwater (SBGW-2 recipe - Matrix 2 conditions). Lower plot presents same data as upper plot on a reduced vertical scale to show detail at low arsenic concentrations. Percent arsenic removed is based on the average initial arsenic concentration. The WHO and Bangladesh maximum allowable arsenic limits are shown as dashed and dotted lines respectively. Error bars represent the variability between identical batch tests. 
ble 3.3. For $j=0.30-1.1 \mathrm{~mA} / \mathrm{cm}^{2}$, the final concentration is $<7 \mu \mathrm{g} / \mathrm{L}(99 \%$ arsenic removal), reaching $<5 \mu \mathrm{g} / \mathrm{L}$ in the case of $j=1.1 \mathrm{~mA} / \mathrm{cm}^{2}$. This demonstrates the ability of ECAR treatment to reduce arsenic below the WHO limit of $10 \mu \mathrm{g} / \mathrm{L}$ in synthetic Bangladesh groundwater with low phosphate and silicate (SBGW-1). Treatment at $j=0.07 \mathrm{~mA} / \mathrm{cm}^{2}$ did not reach the WHO limit after passing a charge density of $q=$ $25 \mathrm{C} / \mathrm{L}\left(\mathrm{As}_{\text {final }}=19 \mu \mathrm{g} / \mathrm{L}\right)$. However, looking at the behavior of arsenic concentration as a function of charge density (Figure $3.12 ; j=0.07 \mathrm{~mA} / \mathrm{cm}^{2}$ data points connected with a line for emphasis), it seems likely that the WHO limit would be reached with the addition of more charge.

Results of "blank" tests (i.e. $j \approx 0 \mathrm{~mA} / \mathrm{cm}^{2}$ ) are also listed in Table 3.3. For blank tests, electrodes were in contact with the water for a time $t_{C P}$, but not external voltage was applied. In the case of $t_{C P}=0 \mathrm{~min}$, there was no electrode contact and treatment went directly to the mixing stage $\left(t_{M}=60 \mathrm{~min}\right)$ followed by filtration. In this case, only $8 \%$ of the arsenic was removed, reaching a final concentration well above the Bangladesh legal limit of $50 \mu \mathrm{g} / \mathrm{L}\left(\operatorname{As}_{\text {final }}=530 \mu \mathrm{g} / \mathrm{L}\right)$. This demonstrates the inability of filtration alone to remove arsenic oxyanions from groundwater. Electrode contact for $t_{C P}=50$ min was also insufficient to reach either the Bangladesh or WHO limit $\left(\mathrm{As}_{\text {final }}\right.$ $=93 \mu \mathrm{g} / \mathrm{L})$, though $84 \%$ of the arsenic was removed. This removal was likely due to EGA generated from natural rates of corrosion. Current during treatment fluctuated from $0.0004-0.007 \mathrm{~mA} / \mathrm{cm}^{2}$, indicating a positive corrosion rate. The much higher removal achieved after treatment at $j=1.1 \mathrm{~mA} / \mathrm{cm}^{2}$ demonstrate the effectiveness of ECAR over technologies based on natural rates of rusting. 
Residual arsenic concentrations after batch tests in SBGW-2 are listed in Table 3.4. Final arsenic concentrations for $j=0.02-5 \mathrm{~mA} / \mathrm{cm}^{2}$ are $\leq 10 \mu \mathrm{g} / \mathrm{L}$, reaching $<2 \mu \mathrm{g} / \mathrm{L}$ for $j=0.02 \mathrm{~mA} / \mathrm{cm}^{2}$. This demonstrates the ability of ECAR treatment to reduce arsenic levels to $<2 \mu \mathrm{g} / \mathrm{L}$ - well below the WHO limit of $10 \mu \mathrm{g} / \mathrm{L}$ - in synthetic Bangladesh groundwater with relevant levels of phosphate and silicate. This demonstration is more significant than SBGW-1 because the SBGW-2 is more representative of Bangladesh groundwater, specifically with respect to solutes that compete with arsenic. Given the high levels of competitive ions in SBGW-2 (including more phosphate and silicate combined than $80 \%$ of contaminated wells in Bangladesh - see Section 3.2.3), these results strongly suggest that ECAR will perform equally well in Bangladesh.

For $j=10-100 \mathrm{~mA} / \mathrm{cm}^{2}$ treatment in SBGW-2 (Table 3.4) arsenic concentrations were quite close to the WHO limit at the highest charge density, $q=175 \mathrm{C} / \mathrm{L}$ $\left(\operatorname{As}_{\text {final }}<15 \mu \mathrm{g} / \mathrm{L}\right)$. Looking at the arsenic concentration as a function of charge density for $j=10-100 \mathrm{~mA} / \mathrm{cm}^{2}$ (Figure 3.13), one sees that concentration is still falling at the end of each run. This suggests that the WHO limit would be reached with the addition of some small amount of charge density.

The arsenic removal capability of ECAR in groundwater with bicarbonate, silicate, and phosphate as well as both As[III] and As[V] is extraordinary. Most chemical adsorbents used in filters in Bangladesh report reducing arsenic to $20-50 \mu \mathrm{g} / \mathrm{L}$, below the Bangladesh legal limit but above the WHO recommended safe limit. These results demonstrate that ECAR can be an effective even in regions of Bangladesh with high levels of phosphate and silicate as well as As[III]. 


\subsubsection{Effect of charge density}

Charge density controls the amount of iron dissolved into solution during the dosing stage of ECAR, and hence the amount of EGA generated. Figures 3.12 and 3.13 show the residual arsenic concentration as a function of charge density from Matrix 1 and Matrix 2 batch experiments respectively. It is apparent from both figures that more charge density leads to more arsenic removal. It is also apparent that the arsenic removal capacity (i.e. the slope of Figure 3.12 or 3.13 ) decreases significantly with the residual arsenic concentration. This behavior is likely due a decrease in adsorption capacity of iron (hydr)oxides at low arsenic concentrations (recall that iron (hydr)oxides typically obey a Langmuir isotherm, discussed in Section 2.4.1). Recall however that the operating costs depend on the average arsenic removal capacity over treatment, not the instantaneous removal capacity. Thus it is the total charge requirement to meet either the Bangladesh or WHO limit that is relevant for Bangladesh. The total charge density required to reach the Bangladesh and WHO limit ( $q_{B D e s h}$ and $q_{W H O}$ respectively) has been estimated in Table 3.5 based on Figures 3.12 and 3.13. In cases where data did not extend far enough in charge density for arsenic to reach the WHO limit, nearby points (i.e. $<9 \mu \mathrm{g} / \mathrm{L}$ above the WHO limit) were linearly extrapolated from the last two or three measured data points. In all cases, extrapolation was $<20 \%$ of the distance between the last two data points. The value of $q_{W H O}$ was used to calculate the arsenic removal capacity and iron capacity as $\mathrm{Fe}^{3+}$ (i.e. $\mu \mathrm{g}$ arsenic removed per $\mathrm{mg}$ of iron dissolved assuming all iron enters solution as $\mathrm{Fe}^{3+}$ ) in Table 3.5. Also calculated is an estimate of the current processing time required to reduce arsenic to the WHO limit based on Equation 2.34 
Table 3.5: Performance metrics to reach the Bangladesh arsenic limit (50 $\mu \mathrm{g} / \mathrm{L})$ and the WHO arsenic limit $(10 \mu \mathrm{g} / \mathrm{L})$ based on ECAR batch tests in synthetic Bangladesh groundwater containing a 1:1 ratio of As[III]:As[V]. Arsenic removal capacity, iron capacity (assuming all is dissolved as $\mathrm{Fe}^{3+}$ ), and total treatment time have been calculated based on the WHO limit and data in Figures 3.12 and 3.13.

\begin{tabular}{|c|c|c|c|c|c|c|c|c|c|}
\hline \multirow[b]{2}{*}{$\begin{array}{l}\text { Water } \\
\text { Type }\end{array}$} & \multirow[b]{2}{*}{$\begin{array}{c}\mathrm{j} \\
\left(\mathrm{mA} / \mathrm{cm}^{2}\right)\end{array}$} & \multirow[b]{2}{*}{$\begin{array}{c}\mathrm{q}_{B D e s h}{ }^{\mathrm{a}} \\
(\mathrm{C} / \mathrm{L})\end{array}$} & \multicolumn{5}{|c|}{ Based on WHO limit } & \multirow[b]{2}{*}{$\begin{array}{c}t_{M} \mathrm{~g} \\
(\min )\end{array}$} & \multirow[b]{2}{*}{$\begin{array}{c}\text { Total } \\
\text { Treatment } \\
\text { Time }^{\mathrm{h}} \\
(\mathrm{Hrs})\end{array}$} \\
\hline & & & $\begin{array}{c}\mathrm{q}_{W H O^{\mathrm{b}}} \\
(\mathrm{C} / \mathrm{L})\end{array}$ & $\begin{array}{c}\text { As } \\
\text { Removed }^{\mathrm{c}} \\
(\mu \mathrm{g} / \mathrm{L})\end{array}$ & $\begin{array}{l}\text { As Removal } \\
\text { Capacity }^{\mathrm{d}} \\
\left(\mu \mathrm{g}-\mathrm{As}_{r e m}\right. \\
\text { /C) }\end{array}$ & $\begin{array}{c}\text { Iron Capacity } \\
\text { as Fe }^{3+} \\
\left(\mu \mathrm{g}-\mathrm{As}_{r e m}\right. \\
\left./ \mathrm{mg}-\mathrm{Fe}^{3+}\right)\end{array}$ & $\begin{array}{l}t_{C P^{f}} \\
(\min )\end{array}$ & & \\
\hline \multicolumn{10}{|l|}{ Matrix 1} \\
\hline SBGW-1 & 1.1 & 50 & 70 & 570 & 8.1 & 42 & 8.99 & 60 & 1.15 \\
\hline SBGW-1 & 0.70 & 50 & 70 & 560 & 8.0 & 42 & 14.12 & 60 & 1.24 \\
\hline SBGW-1 & 0.30 & 50 & 70 & 570 & 8.1 & 42 & 32.96 & 60 & 1.55 \\
\hline SBGW-1 & 0.07 & 15 & $25^{\mathrm{i}}$ & 580 & $22^{\mathrm{j}}$ & $116^{\mathrm{j}}$ & $52.46^{\mathrm{j}}$ & 60 & 1.87 \\
\hline \multicolumn{10}{|l|}{ Matrix 2} \\
\hline SBGW-2 & 100 & 90 & $180^{\mathrm{i}}$ & 560 & $3.0^{\mathrm{j}}$ & $16^{\mathrm{j}}$ & $10.21^{\mathrm{j}}$ & 60 & 1.17 \\
\hline SBGW-2 & 30 & 75 & $160^{\mathrm{i}}$ & 520 & $3.3^{\mathrm{j}}$ & $17^{\mathrm{j}}$ & $8.89^{\mathrm{j}}$ & 60 & 1.15 \\
\hline SBGW-2 & 10 & 75 & $180^{\mathrm{i}}$ & 560 & $3.1^{\mathrm{j}}$ & $16^{\mathrm{j}}$ & $9.92^{\mathrm{j}}$ & 60 & 1.17 \\
\hline SBGW-2 & 5.0 & 75 & 175 & 510 & 2.9 & 15 & 17.68 & 60 & 1.29 \\
\hline SBGW-2 & 1.1 & 55 & 150 & 550 & 3.7 & 19 & 68.87 & 60 & 2.15 \\
\hline SBGW-2 & 0.02 & 15 & 25 & 560 & 22 & 116 & 416.67 & 60 & 7.94 \\
\hline \multicolumn{10}{|l|}{ Other ${ }^{\mathrm{k}}$} \\
\hline SBGW-2 & 1.1 & 25 & 50 & 560 & 11 & 58 & 832.50 & 60 & 14.88 \\
\hline
\end{tabular}

a Charge density required to reduce arsenic to the Bangladesh legal limit of $50 \mu \mathrm{g} / \mathrm{L}$.

b Charge density required to reduce arsenic to the WHO limit of $10 \mu \mathrm{g} / \mathrm{L}$.

c Arsenic removed to reach the WHO limit $(10 \mu \mathrm{g} / \mathrm{L})$ based on the average initial arsenic concentration.

$\mathrm{d}$ Arsenic removal capacity is the average $\mu \mathrm{g}$ arsenic removed per Coulomb to reduce arsenic to the WHO limit $(10 \mu \mathrm{g} / \mathrm{L})$.

e This is the average amount arsenic removed per $\mathrm{Fe}^{3+}$ added to the water to reduce arsenic to the WHO limit $(10 \mu \mathrm{g} / \mathrm{L})$, assuming that all charge is used to oxidize $\mathrm{Fe}(0)$ to $\mathrm{Fe}^{3+}$ (based on faraday's law - Equation 2.19).

f $t_{C P}$ is the estimated current processing time required to reach $\mathrm{q}_{W H O}$ based on Equation 2.34 and the same experimental conditions (i.e. the same A/V).

$\mathrm{g}$ The mixing time, $t_{M}$, is the same as that used for batch tests since the value of $q_{W H O}$ is based on batch test data (and hence based on the choice of $t_{M}$ ).

$\mathrm{h}$ Total ECAR treatment time to reach the WHO limit $(10 \mu \mathrm{g} / \mathrm{L})$ assuming $t_{f i l t}=0$ min. Total treatment time is $T_{\text {treat }}=t_{C P}+t_{M}+t_{\text {filt }}$.

i This value was linearly extrapolated past existing data using the last two or three data points. In each case, the extrapolated distance was $<20 \%$ of the distance between the last two points and the final measured concentration was within $10 \mu \mathrm{g} / \mathrm{L}$ of the WHO limit.

j Based on data extrapolated just beyond the final measured batch test (see previous note).

$\mathrm{k}$ Slow $t_{C P}$ experiment, described in Section 3.3.4. 
(plugging in $q_{W H O}$ and the same value of $\mathrm{A} / \mathrm{V}$ used in the original batch test). Total treatment time to reach the WHO limit is calculated using the same value of $t_{M}$ used in batch tests (since the determination of $q_{W H O}$ from batch tests depends in part on this parameter).

For Matrix 1 tests in SBGW-1, the charge density required for removal to the WHO limit falls between $q_{W H O}=25-70 \mathrm{C} / \mathrm{L}$ depending on current density. For $j=$ $0.30-1.1 \mathrm{~mA} / \mathrm{cm}^{2}$, the charge density requirement is the same, and indeed, removal appears to follow a very similar path for these current densities in Figure 3.12. In this current density range, charge density is a more important factor in removal than current density, agreeing with the findings of Chen et al. (2000) and Kumar et al. (2004). Arsenic removal capacity in this region is around $8 \mu \mathrm{g}-\mathrm{As}_{\mathrm{rem}} / \mathrm{C}$. It is difficult to interpret the much higher removal capacity of $22 \mu \mathrm{g}-\mathrm{As}_{\mathrm{rem}} / \mathrm{C}$ extrapolated from $j=0.07 \mathrm{~mA} / \mathrm{cm}^{2}$ data. From the upper plot of Figure 3.12 (data for $j=0.07$ macmsq connected with lines for emphasis), it appears that the $j=0.07 \mathrm{~mA} / \mathrm{cm}^{2}$ data would follow nicely along the path of other current densities if not for the last data point near $q=25 \mathrm{C} / \mathrm{L}$. From Table 3.3, we see that only one batch tests at this point is available. Further batch tests at higher charge density are needed to determine if current density is affecting removal at $j=0.07 \mathrm{~mA} / \mathrm{cm}^{2}$.

For Matrix 2 tests in SBGW-2, the charge density required to reach the WHO limit varies from $q_{W H O}=25-180 \mathrm{C} / \mathrm{L}$. Arsenic removal capacity is similar for current density range $j=1.1-100 \mathrm{~mA} / \mathrm{cm}^{2}$. However, at the lowest current density, $j=$ $0.02 \mathrm{~mA} / \mathrm{cm}^{2}$, removal capacity is $5-6$ times higher. Unlike the case of $j=0.07 \mathrm{~mA} / \mathrm{cm}^{2}$ 
in SBGW-1, data for $j=0.02 \mathrm{~mA} / \mathrm{cm}^{2}$ extends far above the charge density required to reach the WHO limit (Figure 3.12). This clearly demonstrates that charge density is not the only predictor of arsenic removal capacity in ECAR. In light of this behavior, it is likely that the increase in removal capacity calculated from extrapolated values for $j=0.07 \mathrm{~mA} / \mathrm{cm}^{2}$ is real, and removal capacity is progressively larger at lower current densities.

\subsubsection{Effect of current density}

As discussed in Sections 1.4.3 and 2.5.5, investigators have reported mixed results for the influence that current density has on the efficiency of electrocoagulation processes. For arsenic removal with iron electrodes, Kumar et al. (2004) and Gomes et al. (2007) reported no change in removal efficiency over a current density range of $j=0.65$ $1.53 \mathrm{~mA} / \mathrm{cm}^{2}(\mathrm{pH} 6-8)$ and $j=3-30 \mathrm{~mA} / \mathrm{cm}^{2}(\mathrm{pH} 2.4$ ) respectively. For the removal of other contaminants, Pouet \& Grasmick (1995) reported that current density can affect treatment efficiency, while Chen et al. (2000) disagreed, attributing the discrepancy to the fact that charge density was not held constant in experiments of Pouet and Grasmick. All of the above researchers have noted that current density affects treatment time.

As stated in Section 3.3.3, the batch test results presented in Figures 3.12 and 3.13 and performance metrics based on this data (Table 3.5) indicate that current density does have some effect on arsenic removal capacity, though no effect is seen within certain current density ranges. Recall from Section 2.5.2 that the current density is expected to control the composition of iron (hydr)oxides in EGA. Because the composition of 
iron (hydr)oxides depends on a number of other variables (such as $\mathrm{pH}$ and solution composition) as well as the details of a complicated path through Eh-pH space during electrolysis, it is extremely difficult to predict. It is possible that there is no significant change in composition within certain ranges of current density, but large differences between ranges. This could lead to pockets of stability within which, changing the current density has no effect on arsenic removal capacity.

In the lower plot of Figure 3.13, current densities seem to follow a progression in arsenic removal capacity, with $0.02 \mathrm{~mA} / \mathrm{cm}^{2}>1.1 \mathrm{~mA} / \mathrm{cm}^{2}>5.0 \mathrm{~mA} / \mathrm{cm}^{2}>$ $10 \mathrm{~mA} / \mathrm{cm}^{2} \approx 30 \mathrm{~mA} / \mathrm{cm}^{2} \approx 100 \mathrm{~mA} / \mathrm{cm}^{2}$. However, the calculated removal capacity for $j=5.0 \mathrm{~mA} / \mathrm{cm}^{2}$ in Table 3.5 is very close (within $6 \%$ ) to that of $j=10 \mathrm{~mA} / \mathrm{cm}^{2}$. The lower amount of arsenic removed for $j=5.0 \mathrm{~mA} / \mathrm{cm}^{2}$ indicates that the apparent difference seen in Figure 3.13 is due to a lower initial arsenic concentration rather than an actual increase in removal capacity. Taking this into account, the removal capacity in descending order is $0.02 \mathrm{~mA} / \mathrm{cm}^{2}>1.1 \mathrm{~mA} / \mathrm{cm}^{2}>5.0 \mathrm{~mA} / \mathrm{cm}^{2} \approx 10 \mathrm{~mA} / \mathrm{cm}^{2}$ $\approx 30 \mathrm{~mA} / \mathrm{cm}^{2} \approx 100 \mathrm{~mA} / \mathrm{cm}^{2}$. From batch tests in SBGW-1, the removal capacity in descending order is $0.07 \mathrm{~mA} / \mathrm{cm}^{2}>0.30 \mathrm{~mA} / \mathrm{cm}^{2} \approx 0.70 \mathrm{~mA} / \mathrm{cm}^{2} \approx 1.1 \mathrm{~mA} / \mathrm{cm}^{2}$. The removal capacity in SBGW-1 is not directly comparable to the removal capacity in SBGW-2 because the differences in water composition have a direct affect on removal capacity. However, it is possible to combine the data into an expected progression. From comparisons of $j=1.1 \mathrm{~mA} / \mathrm{cm}^{2}$ in SBGW-1 and SBGW-2, removal capacity decreases in SBGW-2 relative to SBGW-1 (reasons for this effect are discussed more below). The removal capacity of $j=0.07$ macmsq in SBGW-1 is the same as that of $j=0.02 \mathrm{~mA} / \mathrm{cm}^{2}$ 
in SBGW-2 - this suggests that the removal capacity of $0.07 \mathrm{~mA} / \mathrm{cm}^{2}$ would be less than that of $j=0.02 \mathrm{~mA} / \mathrm{cm}^{2}$ if both were used on the same water. Similar comparisons yield the following expected progression, now using ranges of current density to indicate pockets of similar removal capacity: $0.02 \mathrm{~mA} / \mathrm{cm}^{2}>0.07 \mathrm{~mA} / \mathrm{cm}^{2}>0.30-1.1 \mathrm{~mA} / \mathrm{cm}^{2}$ $>5.0-100 \mathrm{~mA} / \mathrm{cm}^{2}$. These results are consistent with both Kumar et al. (2004) and Gomes et al. (2007), who found no effect of current density on arsenic removal efficiency within a range of $j=0.64-1.53 \mathrm{~mA} / \mathrm{cm}^{2}$ and $j=3-30 \mathrm{~mA} / \mathrm{cm}^{2}$ respectively. Both of these ranges fall within the current density pockets of stable arsenic removal capacity ${ }^{10}$.

It has been proposed that changes in arsenic removal capacity with current density are due to a change in iron (hydr)oxides composition. However, recall from the Pourbaix diagram of iron (Figure 2.2) as well as Section 2.5.2 that as the cell potential $\left(\mathrm{E}_{H}\right)$ increases, oxygen evolution becomes thermodynamically favorable. Once this occurs, oxygen evolution will compete with iron dissolution for electrons, lowering the current efficiency (Equation 2.21) and effectively producing fewer iron (hydr)oxides per coulomb than possible at lower cell potentials. In this system, Eh is controlled by current density, and thus oxygen evolution would compete for electrons more effectively as the current density increases. Given that the removal capacity between $j=5.0 \mathrm{~mA} / \mathrm{cm}^{2}$ and $100 \mathrm{~mA} / \mathrm{cm}^{2}$ is so similar, oxygen evolution is unlikely, since the effect would become progressively detrimental to arsenic removal with increasing current density. In addition, no decrease in efficiency was seen by Chen et al. (2000) in the current density range $j=1.25-10.89 \mathrm{~mA} / \mathrm{cm}^{2}$ or Gomes et al. (2007) in the range $j=3-30 \mathrm{~mA} / \mathrm{cm}^{2}$,

\footnotetext{
${ }^{10}$ Note that Gomes et al. (2007) looked at arsenic removal in wastewater at $\mathrm{pH} 2.4$. At this low $\mathrm{pH}$, the ranges of current density pockets are likely to be different from those at $\mathrm{pH}=7$, so it may be coincidence that the tested range falls into a pocket of stable removal capacity.
} 
which should have crossed the oxygen evolution threshold around $j=5 \mathrm{~mA} / \mathrm{cm}^{2}$ if the explanation above is correct. In the case of Gomes et al. (2007), this could be due to the low $\mathrm{pH}$ used $(\mathrm{pH}=2.4)$. The thermodynamic threshold of oxygen evolution increases steeply as pH lowers, as demonstrated in Figure 2.2. Polarization curves in synthetic Bangladesh water can detect oxygen evolution if it is occurring (see Section 3.3.10).

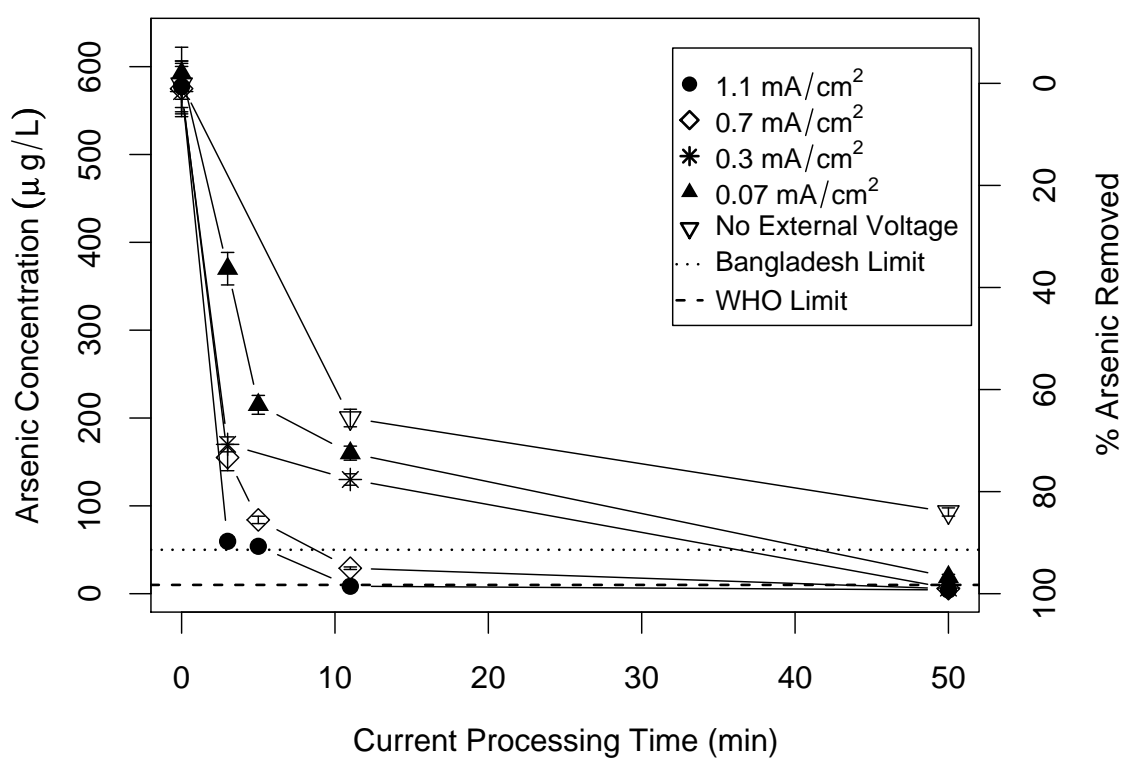

Figure 3.14: Arsenic removal (concentration on left vertical axis; percent arsenic removed on right vertical axis) as a function current processing time for Matrix 1 batch experiments for current densities $j=0.07,0.30,0.70$, and $1.1 \mathrm{~mA} / \mathrm{cm}^{2}$. Also included are batch tests performed with no external voltage (blanks). Percent arsenic removed is based on the average initial arsenic concentration. The WHO and Bangladesh maximum allowable arsenic limits are shown as dashed and dotted lines respectively. Error bars represent the variability between identical batch tests.

Figure 3.14 shows the resulting arsenic concentration for Matrix 1 batch tests 
as a function of current processing time, $t_{C P}$. Consistent with Chen et al. (2000); Kumar et al. (2004); Gomes et al. (2007), an increase in current density decreases the current processing time required to reduce arsenic arsenic. The estimated treatment time to reduce arsenic to the WHO limit is calculated in Table 3.5, revealing a similar progression for SBGW-1 tests. It is difficult to directly compare the treatment times listed for SBGW-2 tests because different values of $\mathrm{A} / \mathrm{V}$ were used to obtain the extreme current densities with available equipment.

Because of the tradeoff between operating variables $j, q, t_{C P}$, and $\mathrm{A} / \mathrm{V}$ (Equation 2.34), $t_{C P}$ was not always constant for a given $q$ dosage between current densities. In fact, $t_{C P}$ varied from minutes to days between $j=100 \mathrm{~mA} / \mathrm{cm}^{2}$ and $j=0.02 \mathrm{~mA} / \mathrm{cm}^{2}$ (see Table 3.2). It is possible that some, or even all, of the increased arsenic removal capacity seen at the lowest current density is due to the drastically increased contact time. In an attempt to isolate the effect of current density, one batch test was run at $j$ $=1.1 \mathrm{~mA} / \mathrm{cm}^{2}, q_{\text {total }}=100 \mathrm{C} / \mathrm{L}$, using the same values of $t_{C P}$ used in the Matrix 2 run for $j=0.02 \mathrm{~mA} / \mathrm{cm}^{2}$ (i.e. a slow- $j=1.1 \mathrm{~mA} / \mathrm{cm}^{2}$ run compared to the relatively fast $j=1.1 \mathrm{~mA} / \mathrm{cm}^{2}$ run from Matrix 2). Results for this run are shown in Figure 3.15 and listed in Table 3.5 under other.

It is clear from Figure 3.15 that some, but not all, of the efficiency gains seen at $j=0.02 \mathrm{~mA} / \mathrm{cm}^{2}$ can be recouped by drastically increasing the current processing time. The charge density required to reach the WHO limit for $j=1.1 \mathrm{~mA} / \mathrm{cm}^{2}$ drops from $q_{W H O}=150 \mathrm{C} / \mathrm{L}$ to $q_{W H O}=50 \mathrm{C} / \mathrm{L}$ with the additional time. This increase in efficiency with additional processing time indicates that either the system is far from 



Figure 3.15: Arsenic removal (concentration on left vertical axis; percent arsenic removed on right vertical axis) as a function of charge density, $q$, for three batch tests; $j=0.02$ $\mathrm{mA} / \mathrm{cm}^{2}$ with $t_{C \text { Ptotal }}=19.4$ hours, $j=1.1 \mathrm{~mA} / \mathrm{cm}^{2}$ with the same $t_{C P \text { total }}=19.4$ hours, and the original Matrix 2 batch test run for $j=1.1 \mathrm{~mA} / \mathrm{cm}^{2}$ using the much shorter $t_{C P t o t a l}=1.2$ hours. Lower plot presents same data as upper plot on a reduced vertical scale to show detail at low arsenic concentrations. Percent arsenic removed is based on the average initial arsenic concentration. The WHO and Bangladesh maximum allowable arsenic limits are shown as dashed and dotted lines respectively. Error bars represent the variability between identical batch tests. 
equilibrium when the batch test is performed quickly, or that oxidation of $\mathrm{As}[\mathrm{III}]$ to $\mathrm{As}[\mathrm{V}]$ is much slower than the processing times used in the original batch test. In either case, increasing the processing time can clearly be used to increase the efficiency of ECAR. It is important to note, however, that $j=0.02 \mathrm{~mA} / \mathrm{cm}^{2}$ still demonstrates a higher arsenic removal capacity than $j=1.1 \mathrm{~mA} / \mathrm{cm}^{2}$ even with processing time is kept constant. This effect is indeed likely due to a significant change in the composition of iron (hydr)oxides produced.

\subsubsection{Effect of current processing time}



Figure 3.16: Arsenic removal (normalized concentration on left vertical axis; percent arsenic removed on right vertical axis) as a function of charge density, $q$, for two batch tests at $j=0.02 \mathrm{~mA} / \mathrm{cm}^{2}$ in which the only difference is a decrease in $t_{C P}$ by $60 \%$ for the Fast test relative to the slow tests. Both batch tests used Matrix 2 procedures and SBGW-2 water with initial arsenic $=600 \mu \mathrm{g} / \mathrm{L}$. 
The current processing time, $t_{C P}$, is generally used only to adjust the charge density (via Equation 2.34). However, $t_{C P}$ may affect arsenic removal capacity in ECAR in a number of different ways that are independent of the applied charge density. As discussed in Section 2.5.2, increasing $t_{C P}$ may allow for increased coagulation relative to mixing due to the presence of the electric field during $t_{C P}$. It could also allow for increased oxidation of As[III] to As[V], a process that does not occur, or occurs much more slowly, during the mixing stage. To determine if any of these processes are significant in ECAR, two batch tests were performed at $j=0.02 \mathrm{~mA} / \mathrm{cm}^{2}$ in which the only change was to decrease $t_{C P}$ by $60 \%$ for each value of charge density $q$ (Figure 3.16 ). The faster run lags in terms of arsenic removal until around $q=25 \mathrm{C} / \mathrm{L}$, at which point it catches up with the slower run. This suggests that there is a separate effect of $t_{C P}$ on arsenic removal until some threshold is reached, at which point the effect disappears. This is consistent with a limitation based on the oxidation of As[III $]$ to As[V] - at some point, oxidation will be complete and no longer delay arsenic removal. If the limitation were due to coagulation, then the fast run would consistently show less arsenic removal.

These results, along with those presented in Figure 3.15 indicate that $t_{C P}$ can affect removal capacity independent of charge density or current density. This is an important finding that indicates (1) the arsenic removal capacity values in Table 3.5 must be taken in the context of their specific $t_{C P}$ values (which are based on the same $\mathrm{A} / \mathrm{V}$ ratio used in batch tests) and (2) $t_{C P}$ should be included as a controlling variable along with $j, q$, and $t_{M}$, that can be tuned to optimize performance metrics in a local water environment. It is worthwhile to note that the differences in $t_{C P}$ between long 
and slow tests is quite large - total treatment to $q=150 \mathrm{C} / \mathrm{L}$ took 19.4 hours in the slow case presented in Figure 3.15, compared to 1.2 hours in the original batch test. The slow case may not be viable in the field if treatment is to occur continuously, or at the longest, overnight. It is not known if smaller changes in $t_{C P}$ significantly affect removal capacity. Future tests are planned to determine the benefit available with smaller time losses.

\subsubsection{Effect of phosphate and silicate on arsenic removal}

It is difficult to directly measure the effect that increased levels of phosphate and silicate in the water have on arsenic removal using this dataset because the Matrix 1 and Matrix 2 procedures utilized different surface-area-to-volume (A/V) ratios, and consequently different current processing times. In addition, the Matrix 1 experiments used a separated cathode cell, which will speed up the net oxidation of As[III] relative to a single chamber cell, since reduction of $\mathrm{As}[\mathrm{V}]$ back to $\mathrm{As}[\mathrm{III}]$ is no longer possible. Thus only a general idea of the effect of silicate and phosphate on arsenic removal via ECAR can be given.

Figure 3.17 shows the final arsenic concentration as a function of charge density, $q$, after ECAR treatment of low phosphate, zero silicate synthetic Bangladesh groundwater (SBGW-1) and average phosphate, average silicate synthetic Bangladesh groundwater (SBGW-2) at current density $j=1.1 \mathrm{~mA} / \mathrm{cm}^{2}$. From the figure, a higher charge density is required to achieve the WHO limit $(10 \mu \mathrm{g} / \mathrm{L})$ of arsenic in SBGW-2 water. For low phosphate, zero silicate water, the first measured point with final arsenic less than 10 


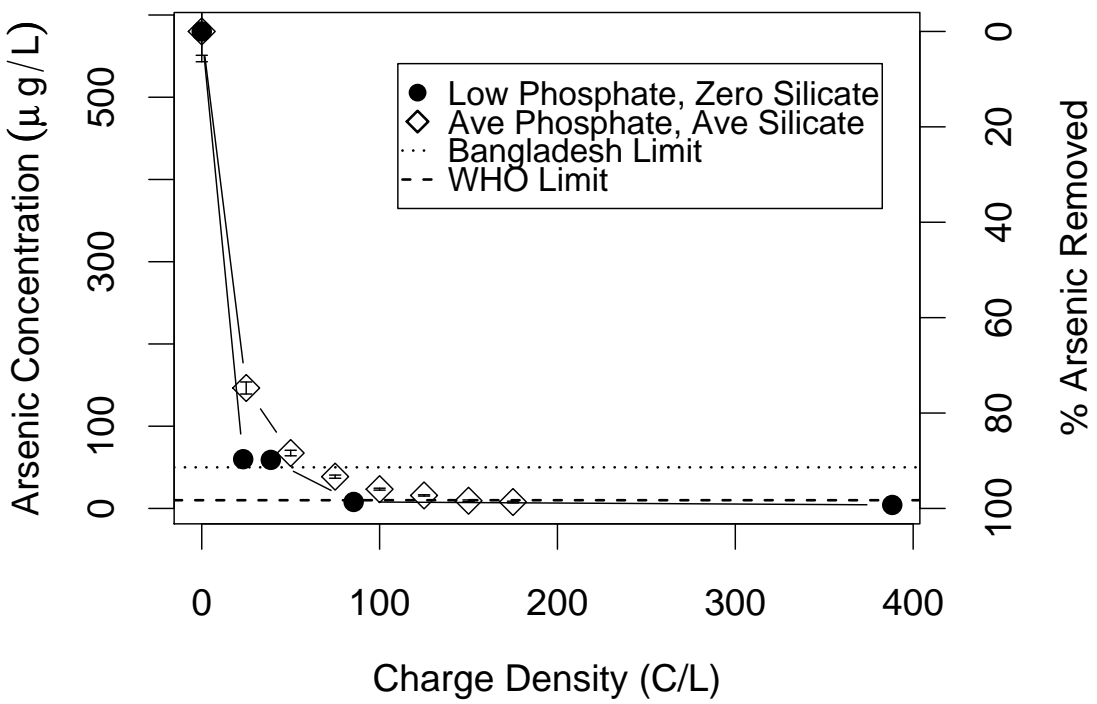

Figure 3.17: Arsenic removal (concentration on left vertical axis; percent arsenic removed on right vertical axis) as a function of charge density, $q$, for low phosphate, zero silicate synthetic Bangladesh groundwater (SBGW-1, using Matrix 1 procedure) and average phosphate, average silicate synthetic Bangladesh groundwater (SBGW-2, using Matrix 2 procedure). ECAR treatment occured at $j=1.1 \mathrm{~mA} / \mathrm{cm}^{2}$ in both cases. All data has been normalized to have an initial arsenic concentration of $580 \mu \mathrm{g} / \mathrm{L}$ for comparison purposes. Lines connecting the data points have been added to guide the eye only. The WHO and Bangladesh maximum allowable arsenic limits are shown as dashed and dotted lines respectively. 
$\mu \mathrm{g} / \mathrm{L}$ is $q=85 \mathrm{C} / \mathrm{L}$, whereas $q=150 \mathrm{C} / \mathrm{L}$ is required in average phosphate, average silicate water, an increase of $76 \%$. From Faraday's law (Equation 2.19), this corresponds also to an increased dosage of Fe[III] or Fe[II] by $76 \%$, or 1.76 times, as well. SBGW-2 water contains $19.5 \mathrm{mg} / \mathrm{L} \mathrm{Si}$ and $1.3 \mathrm{mg} / \mathrm{L} \mathrm{P}$ (see Table 3.1), compared to $0 \mathrm{mg} / \mathrm{L}$ Si and 0.01 $\mathrm{mg} / \mathrm{L} \mathrm{P}$ in SBGW-1. The ratio of Si:Astot in $\mathrm{SBGW-2}$ is $\approx 33: 1$ and that of $\mathrm{P}: \mathrm{As}_{\text {tot }}$ is $\approx 2: 1$.

Recall from Section 2.4.6 that Roberts et al. (2004) investigated the amount of $\mathrm{Fe}[\mathrm{II}]$ and $\mathrm{Fe}[\mathrm{III}]$ required in coprecipitation experiments to reduce $500 \mathrm{ppb}$ of $\mathrm{As}[\mathrm{III}]$ and $\mathrm{As}[\mathrm{V}]$ to below $50 \mathrm{ppb}$ with and without $30 \mathrm{mg} / \mathrm{L} \mathrm{Si}\left(\mathrm{a} \mathrm{Si}: \mathrm{As}_{\text {tot }}\right.$ ratio of 60:1) and 3 mg/L P (a P:Astot ratio of 6:1). Over 2.5 and 7 times the Fe[III] was needed for As[III] and $\mathrm{As}[\mathrm{V}]$ respectively in the presence of $\mathrm{Si}$ and $\mathrm{P}$. About 1.5 and 5 times the Fe[II] was needed for As[III] and As[V] respectively. If the increase in $\mathrm{Si}$ and $\mathrm{P}$ produced a linear detrimental effect on arsenic removal, the Roberts et al. (2004) data would imply 1.253.5 times the $\mathrm{Fe}[\mathrm{III}]$ or $0.75-2.5$ times the $\mathrm{Fe}[\mathrm{II}]$ additions would be required to remove arsenic from SBGW-2 water (which has approximately half the Si and P added per mg As as the Roberts et al. (2004) data, along with containing half As[III] and half As[V] initially). In this case, the increase in dosage required by ECAR in the presence of $\mathrm{Si}$ and $\mathrm{P}$ is similar to that required by coprecipitation with $\mathrm{Fe}[\mathrm{II}]$ or $\mathrm{Fe}[\mathrm{III}]$. In addition, SBGW-2 has bicarbonate, which can act with Si to enhance the detrimental effect of P (Section 2.4.6). We can nevertheless say that the detrimental effect on arsenic removal via ECAR in the presence of $\mathrm{Si}$ and $\mathrm{P}$ is consistent with the detrimental effect seen in arsenic removal via coprecipitation with Fe[II] or Fe[III]. 


\subsubsection{Parameter trends, tradeoffs, and implications for Bangladesh}

The ECAR parameters $j, q, t_{C P}$, and $t_{M}$ were all found to have an effect on arsenic removal capacity in synthetic Bangladesh groundwater at $\mathrm{pH}=7$. Removal capacity was found to decrease with current density excepting certain ranges of stability defined by: $0.02 \mathrm{~mA} / \mathrm{cm}^{2}>0.07 \mathrm{~mA} / \mathrm{cm}^{2}>0.30-1.1 \mathrm{~mA} / \mathrm{cm}^{2}>5.0-100 \mathrm{~mA} / \mathrm{cm}^{2}$. Increasing the charge density decreases the current processing time if $\mathrm{A} / \mathrm{V}$ is held constant. Increasing the charge density requires either a higher operating current (and hence a higher operating voltage, leading to increased energy consumption) or an increase in the active electrode surface area.

Arsenic removal was found to increase with charge density, $q$. The charge density required to reduce $550-580 \mu \mathrm{g} / \mathrm{L}$ arsenic to below the WHO limit with $t_{M}=$ $60 \min$ was $q=25-180 \mathrm{C} / \mathrm{L}$. The charge density requirement was found to be sensitive to the composition of sample water and operating conditions such as $j$ and $t_{C P}$. It should not be taken as an absolute value, but calibrated to the specific water environment and conditions in use. In general, higher arsenic removal capacity implies a lower charge density requirement to reach a given concentration limit. At a given current density, increasing the charge density requires an increase in the current processing time.

Increasing the current processing time was found to increase the arsenic removal capacity independently of $q$. Increasing the mixing time, $t_{M}$ was found to increase arsenic removal capacity rapidly for the first 10 - 15 minutes, and very slowly thereafter (data presented in Section 3.2). Thus there is a tradeoff between removal capacity and treatment time for both $t_{C P}$ and $t_{M}$. Filtration times greater than 3 days (in 
sedimentation tests) were found to increase the arsenic removal capacity slightly at for a large time tradeoff. This effect differs from an increase in $t_{M}$ only because of the lack of stirring during filtration (i.e. increasing $t_{M}$ is likely to be more time efficient).

In batch tests, the highest arsenic removal capacity in SBGW-2 (including relevant levels of bicarbonate, phosphate, and silicate to give a reasonable estimate of performance in Bangladesh) with a final concentration at the WHO limit, was achieved at $j=0.02 \mathrm{~mA} / \mathrm{cm}^{2}, q=25 \mathrm{C} / \mathrm{L}, t_{C P}=416 \mathrm{~min}(\sim 7 \mathrm{hrs}), t_{M}=60 \mathrm{~min}$. Total treatment time ( $8 \mathrm{hrs})$ was just above the target for a community water system $(6-7 \mathrm{hrs})$. In light of the long treatment time, a second effective set of conditions in SBGW-2 was $j=$ $1.1 \mathrm{~mA} / \mathrm{cm}^{2}, q=150 \mathrm{C} / \mathrm{L}, t_{C P}=69 \mathrm{~min}, t_{M}=60 \mathrm{~min}$. The total treatment time was 2.15 hrs. Both of these parameter sets have advantages (the first in terms of operating costs, and the second in terms of treatment time) and choosing between them would require more detailed knowledge on the importance of reduced operating costs compared to treatment time. The actual operating conditions should also take into account relevant safety factors.

\subsubsection{Adsorption using post-synthesis ECAR-generated adsorbent}

In order to explore the significance of producing iron (hydr)oxides in-situ with arsenic during ECAR, experiments were performed using iron (hydr)oxides that were not generated in-situ. Post-synthesis ECAR-generated iron (hydr)oxides adsorbent, or PS-EGA adsorbent, was created by running the ECAR dosing process in synthetic Bangladesh groundwater containing no arsenic. PS-EGA was placed in contact with ar- 
senic after being freshly made (fresh-PS-EGA) as well as after being aged for 60 minutes (aged-PS-EGA). Figure 3.18 shows the adsorption achieved by both fresh- and aged-PSEGA adsorbent as a function of contact time. It is clear that fresh-PS-EGA adsorbent removes arsenic much more quickly than the aged-PS-EGA adsorbent, leading to a final arsenic value that is 10 times lower after 120 minutes of contact time. In terms of removal capability, fresh-PS-EGA adsorbent is capable of reducing arsenic to below the Bangladesh limit after about 45 minutes of contact time, as well as approach the WHO limit at 120 minutes (final arsenic concentration was $22 \mu \mathrm{g} / \mathrm{L}$ ). However, aged-PS-EGA adsorbent comes nowhere near even the Bangladesh limit after 120 minutes of contact (final arsenic concentration was $190 \mu \mathrm{g} / \mathrm{L}$ ). This implies that the age of PS-EGA adsorbent has a huge impact on the final arsenic concentration achievable using post-synthesis ECAR-generated adsorbents. Even after aging for only 60 minutes, treatment of synthetic Bangladesh groundwater to below the Bangladesh legal limit becomes impossible without (perhaps) a large increase in adsorbent added or (possibly) a large increase in contact time. Since 60 minutes is too short to transport pre-formed adsorbent in a useful way, in-situ generation is necessary to achieve the arsenic removal capacity and arsenic removal capability of ECAR.

It appears from Figure 3.18 that the arsenic concentration for aged-PS-EGA plateaus after about 80 minutes of contact, while the arsenic concentration for freshPS-EGA continues to decrease up to 120 minutes of contact. Figure 3.19 and Table 3.6 show results after 1-2 days of quiescent contact time following the mixing period (in each case, samples were filtered using $0.1 \mu \mathrm{m}$ membranes before arsenic analysis). From this 


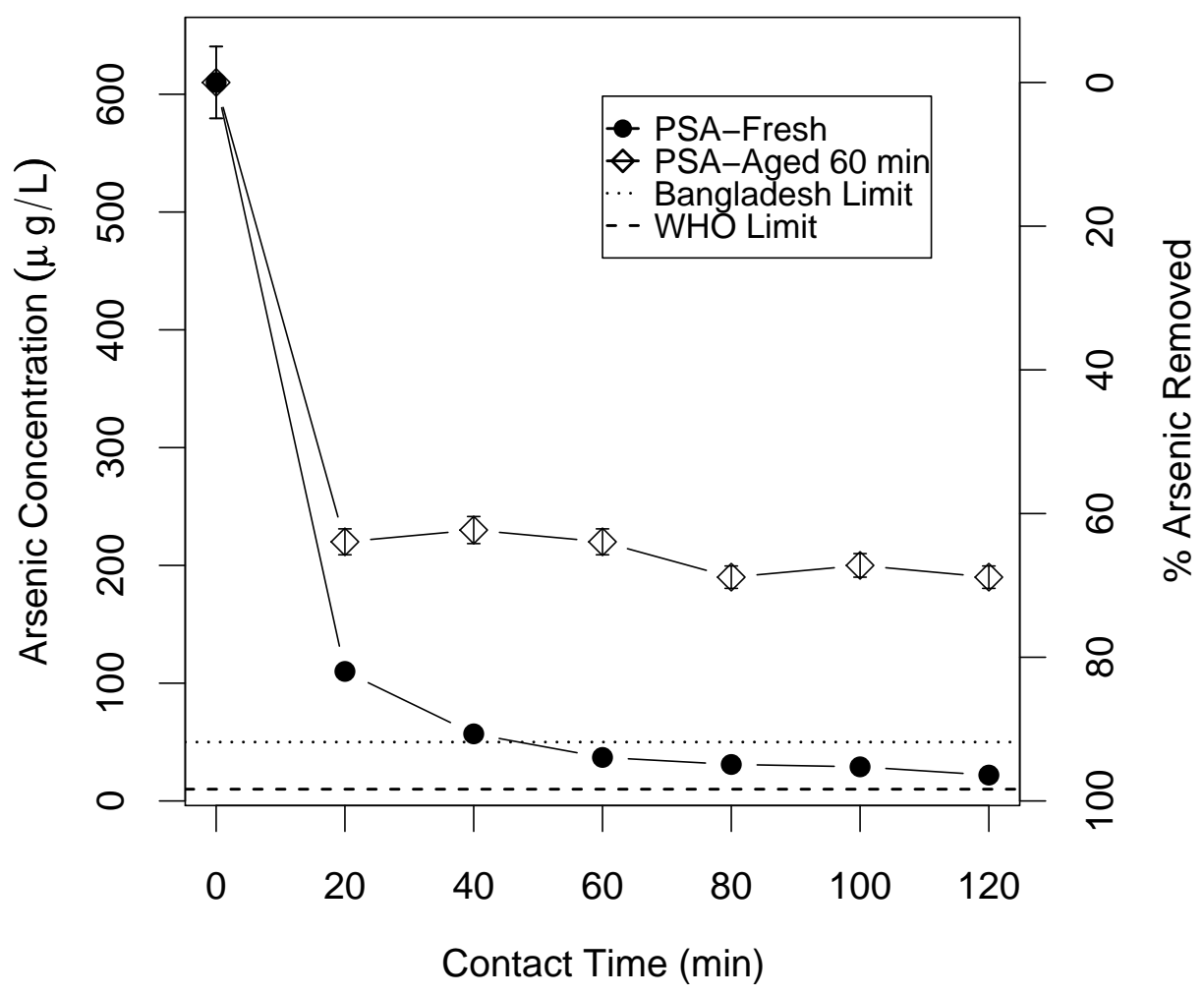

Figure 3.18: Arsenic removal (concentration on left vertical axis; percent arsenic removed on right vertical axis) as a function of contact time for PSA experiments using post-synthesis ECAR-generated adsorbents, both freshly prepared (PSA-Fresh) and aged for 60 minutes (PSA-Aged $60 \mathrm{~min}$ ). Adsorbent was generated using ECAR operating conditions $j=5.0 \mathrm{~mA} / \mathrm{cm}^{2}, q=175 \mathrm{C} / \mathrm{L}, t_{C P}=17.27 \mathrm{~min}$ in arsenic-free SBGW-2 water. Note that the initial arsenic level is estimated based on the spiked amount (see Section 3.2.8). Percent arsenic removed is based on the average initial arsenic concentration. The WHO and Bangladesh maximum allowable arsenic limits are shown as dashed and dotted lines respectively. Error bars represent the variability between identical batch tests. 


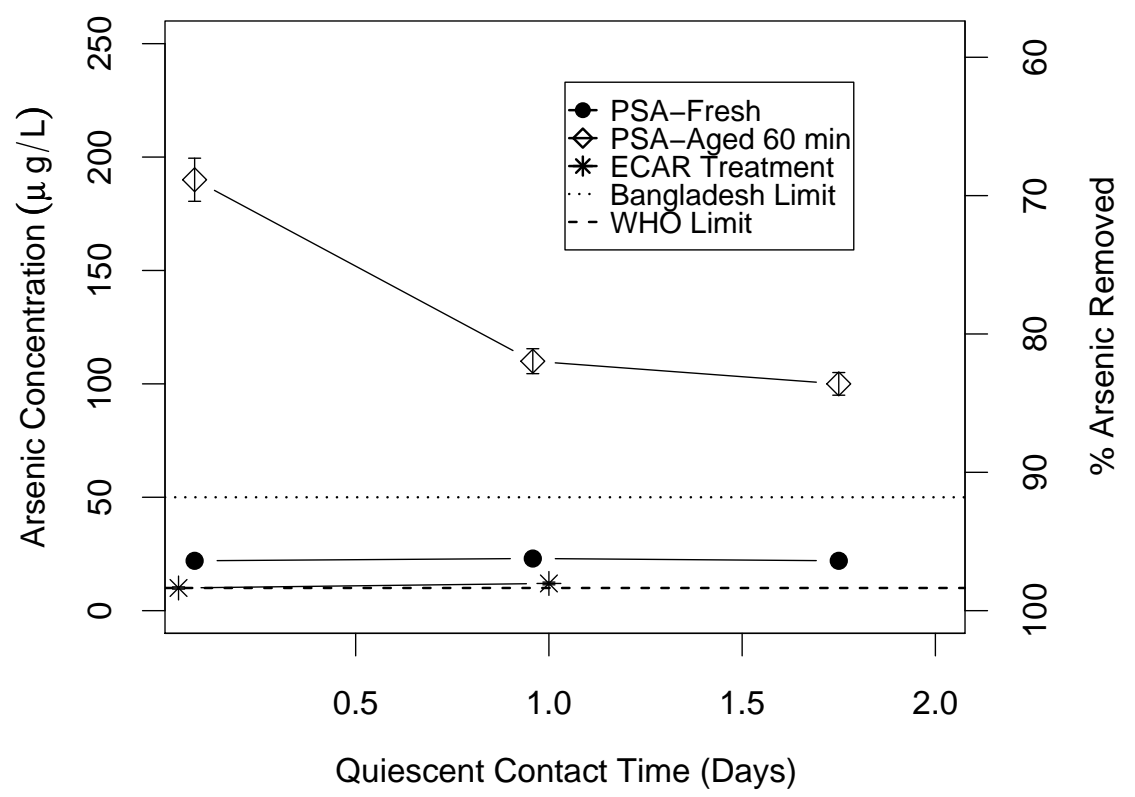

Figure 3.19: Arsenic removal (concentration on left vertical axis; percent arsenic removed on right vertical axis) as a function of quiescent contact time for PSA experiments using post-synthesis ECAR-generated adsorbents, both freshly prepared (PSA-Fresh) and aged for 60 minutes (PSA-Aged $60 \mathrm{~min}$ ), and compared to ECAR treatment at the same current density $\left(j=5.0 \mathrm{~mA} / \mathrm{cm}^{2}\right)$ and a similar current processing time $\left(t_{C P}=16.33\right.$ min) as that used to generate the PSA adsorbents. This is a continuation of Figure 3.18 (note however the change in $\mathrm{x}$-axis units). In all cases, quiescent contact began after a period of active mixing $\left(t_{M}=60 \mathrm{~min}\right.$ in the case of ECAR treatment, and $t_{M}=120$ minutes in the case of PSA experiments). Initial arsenic concentration in all three cases was $\mathrm{As}_{t o t}=590 \pm 40 \mu \mathrm{g} / \mathrm{L}$. Percent arsenic removed is based on the average initial arsenic concentration. The WHO and Bangladesh maximum allowable arsenic limits are shown as dashed and dotted lines respectively. Error bars represent the variability between identical batch tests. 
Table 3.6: Comparison of arsenic removal capability and arsenic removal capacity using ECAR treatment versus treatment with freshly made ( $P S A$-fresh) and aged $60 \mathrm{~min}$ (PSA-aged) post-synthesis ECAR-generated adsorbents. Arsenic removal capacity has been calculated assuming that all of the applied charge (during ECAR treatment or ECAR-generation of adsorbent) is used to form $\mathrm{Fe}^{3+}$ ions in solution.

\begin{tabular}{|c|c|c|c|c|c|c|c|}
\hline Treatment & $\begin{array}{c}\text { Initial } \\
\text { As } \\
(\mu \mathrm{g} / \mathrm{L})\end{array}$ & $\begin{array}{l}\text { Residual } \\
\text { As at } \\
60 \text { min } \\
(\mu \mathrm{g} / \mathrm{L})\end{array}$ & $\begin{array}{l}\text { Residual } \\
\text { As at } \\
120 \text { min } \\
(\mu \mathrm{g} / \mathrm{L})\end{array}$ & $\begin{array}{c}\text { Residual } \\
\text { As at } \\
1 \text { day settle } \\
(\mu \mathrm{g} / \mathrm{L})\end{array}$ & $\begin{array}{c}\text { Residual } \\
\text { As at } \\
2 \text { day settle } \\
(\mu \mathrm{g} / \mathrm{L})\end{array}$ & $\begin{array}{c}\text { As Removal } \\
\text { Capacity }^{\mathrm{a}} \text { at } \\
60 \mathrm{~min} \\
\left(\mu \mathrm{g}-\mathrm{As}_{r e m} /\right. \\
\left.\mathrm{mg}-\mathrm{Fe}^{3+}\right) \\
\end{array}$ & $\begin{array}{l}\text { As Removal } \\
\text { Capacity }^{\mathrm{a}} \text { At } \\
2 \text { day settle } \\
\left(\mu \mathrm{g}-\mathrm{As}_{r e m} /\right. \\
\left.\mathrm{mg} \mathrm{Fe}^{3+}\right)\end{array}$ \\
\hline $\mathrm{ECAR}^{\mathrm{b}}$ & 540 & 10 & NA & 12 & NA & 18.3 & $18.3^{\mathrm{c}}$ \\
\hline PSA-Fresh & $610^{\mathrm{d}}$ & 37 & 22 & 23 & 22 & 17.0 & 17.4 \\
\hline PSA-Aged & $610^{\mathrm{d}}$ & 220 & 190 & 110 & 100 & 11.5 & 15.1 \\
\hline
\end{tabular}

a Arsenic removal capacity (in $\mu \mathrm{g}$-As-removed $/ \mathrm{mg}^{-} \mathrm{Fe}^{3+}$ ) assumes that all charge added is used to generate $\mathrm{Fe}^{3+}$ ions in solution.

b ECAR treatment used similar operating parameters $\left(j=5.0 \mathrm{~mA} / \mathrm{cm}^{2}, q=175 \mathrm{C} / \mathrm{L}, t_{C P}=16.33 \mathrm{~min}\right)$ as those used to generate PS-EGA adsorbents $\left(j=5.0 \mathrm{~mA} / \mathrm{cm}^{2}, q=175 \mathrm{C} / \mathrm{L}, t_{C P}=17.27 \mathrm{~min}\right)$ and used the same sample water composition (SBGW-2). After 60 minutes of mixing, the ECAR sample was left to quiescent settling, and thus no data is available at 120 minutes of mixing. In addition, data was only taken after 1 day of quiescent settling.

c The arsenic removal capacity here is assumed to be the same as it was after 1 day of quiescent settling, based on showing no change in arsenic between 60 minutes and 1 day of settling (and assuming no change would occur between 1-2 days).

d Initial arsenic values are estimated from spiked amount (see Section 3.2.8 for explanation). 
figure, it is clear that the arsenic concentration for fresh-PS-EGA has reached a plateau, while the arsenic concentration for aged-PS-EGA continues to fall. This is similar to the trend seen in adsorption via coprecipitation compared to PSA. Recall from Section 2.4.5 that adsorption onto pre-formed iron (hydr)oxides (PSA) tends to take longer than adsorption via coprecipitation because aggregates have already formed, and ions must diffuse into the aggregates before steady-state can be achieved. The formation of aggregates is likely the cause of the slower arsenic removal and lower arsenic removal capacity seen in aged-PS-EGA compared to fresh-PS-EGA.

Table 3.6 lists the arsenic concentration and inferred arsenic removal capacity (assuming all charge is used to generate $\mathrm{Fe}^{3+}$ ions) for the PS-EGA adsorbents as well as ECAR treatment. In this comparison, the ECAR treatment used similar operating parameters $\left(j=5.0 \mathrm{~mA} / \mathrm{cm}^{2}, q=175 \mathrm{C} / \mathrm{L}, t_{C P}=16.33 \mathrm{~min}\right)$ as those used to generate PS-EGA adsorbents $\left(j=5.0 \mathrm{~mA} / \mathrm{cm}^{2}, q=175 \mathrm{C} / \mathrm{L}, t_{C P}=17.27 \mathrm{~min}\right)$ and treated the same sample water composition (SBGW-2). Therefore, differences in arsenic removal capacity are not likely due to differences in the iron (hydr)oxides composition of the adsorbent produced or composition differences in the sample. From Table 3.6, one can see that the initial arsenic concentration is a bit lower for the ECAR treatment test compared to the PS-EGA tests, however this difference is almost within measurement error $(10 \%)$ and is automatically normalized during the arsenic removal capacity calculation. One can see from Table 3.6, as well as Figure 3.19, that ECAR treatment performs better than PSEGA adsorbents both in the short term (after the standard 60 minutes of mixing contact) and longer term (after 1-2 days of quiescent contact). Notably however, the performance 
of fresh-PS-EGA is not far behind that of ECAR in terms of arsenic removal capability (reducing to $22 \mu \mathrm{g} / \mathrm{L}$ as opposed to $10 \mu \mathrm{g} / \mathrm{L}$ ) or arsenic removal capacity (17.0 compared

to $\left.18.3 \mu \mathrm{g}-\mathrm{As}_{\mathrm{rem}} / \mathrm{mg}-\mathrm{Fe}^{3+}\right)$. This indicates that only a small relative advantage is gained from arsenic adsorption that occurs during the current processing time of ECAR. This is interesting to note because one process that may occur during electrolysis is enhanced coagulation of forming iron (hydr)oxides particles due to the applied electric field (see Section 2.5.2). Enhanced coagulation would tend to age the generated iron (hydr)oxides (in terms of aggregate formation) at an accelerated rate compared to simple mixing. From the comparison of fresh-PS-EGA to 60 min aged-PS-EGA, a small acceleration in aging could significantly reduce the arsenic removal capacity of the generated adsorbent. This does not seem to be the case, indicating that the iron (hydr)oxides formed during ECAR are not significantly more aggregated due to electrolysis.

It is also interesting to note that the final arsenic concentration in fresh-PSEGA solution does not approach the final concentration due to ECAR treatment, even after 2 days of quiescent contact (Figure 3.19). If the only difference between the two adsorbents were aggregation, the one would expect the final concentration to converge as more time was allowed for diffusion. This indicates that some advantage of in-situ generation during ECAR cannot be made up for using pre-formed adsorbent.

\subsubsection{As[III] removal in ECAR}

It is clear from Matrix 1 and Matrix 2 results (Figures 3.12 and 3.13) that ECAR is capable of reducing both $\mathrm{As}[\mathrm{III}]$ and $\mathrm{As}[\mathrm{V}]$ from $300 \mu \mathrm{g} / \mathrm{L}$ to levels below the 




Figure 3.20: Post-treatment As[III] and As[V] (concentration on left vertical axis; percent arsenic removed on right vertical axis) as a function of charge density, $q$. This batch test was run on SBGW-2 water with operating conditions $j=1.1 \mathrm{~mA} / \mathrm{cm}^{2}, t_{C P}=66.58 \mathrm{~min}$, Matrix 2 procedure. These results were typical of all current densities in Matrix 2 (i.e. $0.02,1.1,5.0,10,30$, and $100 \mathrm{~mA} / \mathrm{cm}^{2}$ ). Lines have been added to guide the eye only. Percent arsenic removed is based on the average initial arsenic concentration. The WHO and Bangladesh maximum allowable arsenic limits are shown as dashed and dotted lines respectively. Error bars represent the variability between identical batch tests. 


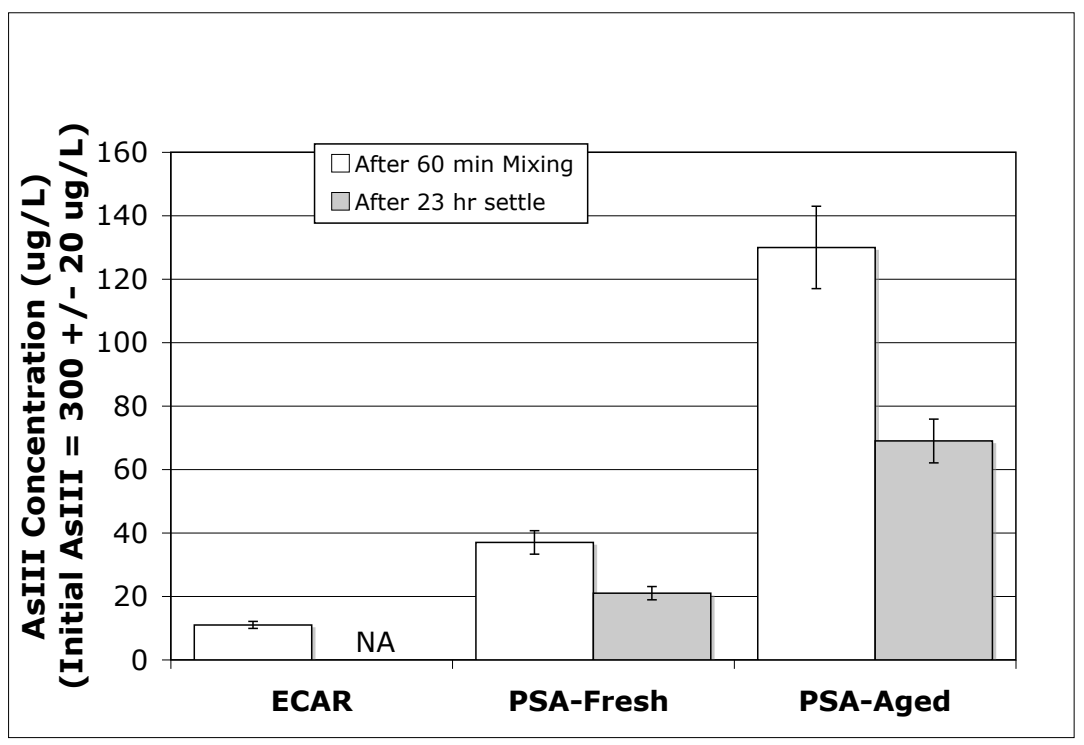

Figure 3.21: Comparison of the final As[III] concentration (initial concentration 300 $\mu \mathrm{g} / \mathrm{L}$ ) for SBGW-2 water after ECAR treatment to adsorption with freshly made post-synthesis ECAR-generated adsorbent (PSA-Fresh) and aged post-synthesis ECARgenerated adsorbent (PSA-Aged). ECAR treatment was performed under similar conditions to the generation of post-synthesis adsorbent. See Section 3.3.8 and Section 3.2.8 for experimental conditions. 
WHO limit of $10 \mu \mathrm{g} / \mathrm{L}$ at $\mathrm{pH}$ 7. Kumar et al. (2004) has attributed similar behavior (using EC with iron) to net oxidation of As[III]. Net electrochemical oxidation of As[III] to $\mathrm{As}[\mathrm{V}]$ is a significant side-reaction that may occur during the electrochemical process in ECAR. Oxidation of As[III] may also occur in parallel with Fe[II] oxidation by dissolved $\mathrm{O}_{2}$ (see Section 2.4.3). Oxidation of As[III] is advantageous if ECAR-generated iron (hydr)oxides have a higher binding affinity for As[V] than As[III]. Recall from Section 2.4.4 that coprecipitation with alum, ferric chloride $\left(\mathrm{Fe}^{3+}\right)$, and ferric sulfate $\left(\mathrm{Fe}^{2+}\right)$ is less efficient for As[III] removal than As[V] under comparable conditions (Hering et al., 1996; Gulledge \& Oconnor, 1973; Sorg \& Logsdon, 1978; Roberts et al., 2004; Meng et al., 2002; Shen, 1973; Leckie et al., 1980; Meng et al., 2000). However, experiments on preformed iron (hydr)oxides, including ferrihydrite and goethite, have been discrepant at arsenic concentrations near those used here $(8 \mu \mathrm{M})$. A crossover $\mathrm{pH}$ (at which the binding strength of $\mathrm{As}[\mathrm{III}]$ and $\mathrm{As}[\mathrm{V}]$ are equal) of 7.0 has been reported using ferrihydrite at concentrations of $13.3 \mu \mathrm{M}$ arsenic (Pierce \& Moore, 1982). Yet, Dixit \& Hering (2003) report a crossover $\mathrm{pH}$ of 8.5 for ferrihydrite at similar arsenic concentrations (see Section 2.4.4 for more discussion). In light of the disagreements in the literature, it is not clear if the ability to reduce As[III] concentrations comes primarily from a high affinity of ECARgenerated iron (hydr)oxides for As[III] at $\mathrm{pH} 7$ or from the net oxidation of As[III] and subsequent adsorption as As[V].

Figure 3.20 shows the $\mathrm{As}[\mathrm{III}]$ and $\mathrm{As}[\mathrm{V}]$ concentrations as a function of charge density during one of the Matrix 2 batch tests (SBGW-2 water, $j=1.1 \mathrm{~mA} / \mathrm{cm}^{2}, t_{M}$ $=60 \mathrm{~min}, q$ and $t_{C P}$ varied). Despite starting from similar initial concentrations, As $[\mathrm{V}]$ 
is reduced to less than $10 \mu \mathrm{g} / \mathrm{L}$ after only $50 \mathrm{C} / \mathrm{L}$ passed, while $\mathrm{As}[\mathrm{III}]$ requires up to $150 \mathrm{C} / \mathrm{L}^{11}$. Even as a lower bound, this implies that $\mathrm{As}[\mathrm{V}]$ is removed more efficiently than As[III] (i.e. requires less charge per mg arsenic removed). Since the composition of iron (hydr)oxides produced during ECAR are a function of the current density used, the results shown would only apply to $j=1.1 \mathrm{~mA} / \mathrm{cm}^{2}$. However, these results were typical of all measured current densities, including $0.02,1.1,5.0,10,30$, and $100 \mathrm{~mA} / \mathrm{cm}^{2}$, implying that $\mathrm{As}[\mathrm{V}]$ is more efficiently removed within the tested current density range. This is consistent with a net oxidation of $\mathrm{As}[\mathrm{III}]$. It is also consistent with a slightly lower binding affinity for As[III], requiring more time, as opposed to more charge, to adsorb to iron (hydr)oxides.

One way to separate electrochemical oxidation of As[III] from adsorption of As[III] and/or oxidation of As[III] via parallel Fe[II] oxidation is to look at the As[III] concentration during post-synthesis ECAR-generated (PS-EGA) adsorbent experiments (Section 3.3.8). In these experiments, As[III] is not present during electrolysis, and so cannot be oxidized by the anode ${ }^{12}$. It can, however, adsorb to the PS-EGA adsorbent as As[III] or oxidize in parallel with Fe[II]. Figure 3.21 compares the As[III] concentration after ECAR treatment (including 60 minutes of mixing) to adsorption onto PS-EGA adsorbent after 60 minutes of contact and 23 hours of quiescent contact. ECAR treatment has been chosen to closely match the conditions of adsorbent generation for PS-EGA

\footnotetext{
${ }^{11}$ Note that these numbers could only be used to calculate a lower bound on the arsenic removal capacity for $\mathrm{As}[\mathrm{V}]$ and $\mathrm{As}[\mathrm{III}]$, since the net $\mathrm{As}[\mathrm{V}]$ concentration may grow during electrolysis if net oxidation of $\mathrm{As}[\mathrm{III}]$ is occurring. It is also possible that the As[V] concentration could lower if net reduction to As[III] is occurring, though this is unlikely.

${ }^{12}$ Oxidation of As[III] could occur without electrolysis, as measured in synthetic Bangladesh groundwater batches during storage (see Section 3.2.3). However, this effect was only significant after 2-3 days of storage.
} 
(see Section 3.3.8). It is clear that less As[III] remains after ECAR treatment than after adsorption with PS-EGA. However, $90 \%$ and $40 \%$ of the As[III] is adsorbed by fresh-PSEGA and aged-PS-EGA respectively within 60 minutes of contact. This clearly indicates that ECAR-generated adsorbent has some affinity for As[III], even in the presence of competing As $[\mathrm{V}]$ as well as background ions present in SBGW-2 water. The As[III] continues to adsorb over 23 hours of quiescent contact. Thus one could argue that the lower levels of As[III] seen during ECAR treatment are entirely due to the increased contact time allowed by arsenic contact during electrolysis (i.e. in the ECAR case, arsenic was in contact with ECAR-generated iron (hydr)oxides for 16 minutes longer during electrolysis). However, even after 23 hours of contact, As[III] levels in the PS-EGA adsorbent have not reached those of ECAR treatment after 76 minutes of contact, indicating that the extra contact time is not the only advantage. This provides evidence that some electrochemical oxidation of $\mathrm{As}[\mathrm{III}]$ is occurring during electrolysis. Oxidation may explain part or all of the advantage of in-situ generation over post-synthesis adsorption see in Section 3.3.8.

\subsubsection{Polarization studies}

Figure 3.22 shows an anodic polarization scan for iron in plain perchlorate solution $\left(0.1 \mathrm{M} \mathrm{KClO}_{4}\right)$ compared to perchlorate plus synthetic Bangladesh groundwater $\left(0.1 \mathrm{M} \mathrm{KClO}_{4}+\mathrm{SBGW}\right)$ at a scan rate of $\nu=0.1 \mathrm{mV} / \mathrm{s}$. The slow scan rate allows the current to come close to equilibrium at each potential step, allowing for some idea of the equilibrium current density that corresponds to each voltage in SBGW (see discussion in 




Figure 3.22: Anodic polarization curve for iron wire electrode in $0.1 \mathrm{M} \mathrm{KClO}_{4}$ (thin line) and $0.1 \mathrm{M} \mathrm{KClO}_{4}+\mathrm{SBGW}$ (thick line) scanned at $\nu=0.1 \mathrm{mV} / \mathrm{s}$. Horizontal lines indicate the current densities used in Matrix 1 (dotted) and Matrix 2 (dashed) experiments. Thermodynamic equilibrium potentials at $25^{\circ} \mathrm{C}$ for appropriate redox couples are shown for $\mathrm{pH}=7$ (in red) - expected of natural groundwater - and $\mathrm{pH}=9$ (in blue) - close to the $\mathrm{pH}$ of the actual scan. A pourbaix diagram composed by Bang et al. (2005a) assuming solid $\mathrm{Fe}, \mathrm{Fe}(\mathrm{OH})_{2}$ and $\mathrm{Fe}(\mathrm{OH})_{3}$ exist was consulted to select appropriate lines at each $\mathrm{pH}$. If concentration appears in the equilibrium calculation, a range of concentrations from $10^{-8} \mathrm{M}$ to $1 \mathrm{M}$ are shown. 
Section 3.2.9). This in turn gives an idea of the interface potential expected in SBGW at a given constant current density. Horizontal lines depict the current densities used in Matrix 1 and Matrix 2 experiments. All scans went fully negative (to -1.4V vs SHE) before the oxidation scan to eliminate any prior oxide layer buildup on the electrode.

A comparison of the scan in plain perchlorate to the scan in perchlorate plus SBGW should reveal electrochemical effects due only to ionic species in the synthetic groundwater. In Figure 3.22, the SBGW polarization scan is shifted anodically by approximately $50 \mathrm{mV}$ compared to the plain perchlorate scan. This shift translates into a large drop in current, especially at potentials $<-0.1 \mathrm{~V}$, suggesting that species in the SBGW are interfering with the dissolution processes on the iron electrode. The drop in current could indicate slower kinetics, a different oxidation product distribution, and/or a compact passive layer inhibiting but not preventing current flow. The shift is too large to be attributed to $\mathrm{pH}$ changes alone $\left(\mathrm{pH}_{f}=9.14\right.$ and $\mathrm{pH}_{f}=9.60$ in plain perchlorate and perchlorate plus SBGW respectively). 0.1M perchlorate at was added to both solutions, and thus the decrease in current is not due to the high resistivity of SBGW. Thus, in addition to the overpotential required to overcome the high resistivity of Bangladesh groundwater, some overpotential will be required overcome the drop in current measured above, further increasing the energy consumption of ECAR treatment. The drop in current relative to plain perchlorate decreases with increasing current density, indicating that this effect is smaller at higher current densities $\left(j>5 \mathrm{~mA} / \mathrm{cm}^{2}\right)$. Thus higher current densities may have some advantage over lower current densities in terms of energy 
consumption ${ }^{13}$.

Notably, no iron passivation is seen within the range of the scan (i.e. up to $\left.10 \mathrm{~mA} / \mathrm{cm}^{2}\right)$. Passivation of iron occurs when an oxide layer builds up on the electrode surface, preventing current from passing. The onset is characterized by a steep decrease in current during oxidation, falling as low as $30-50 \mu \mathrm{A} / \mathrm{cm}^{2}$ in perchlorate solution (Jovanovic \& Hackerman, 1998). The passivation potential of iron in perchlorate solution is typically $0.544 \pm 0.030 \mathrm{~V}$ vs SHE (Jovanovic \& Hackerman, 1998), well above the region corresponding to $j=10 \mathrm{~mA} / \mathrm{cm}^{2}$ in Figure 3.22. Unfortunately, the scan did not reach current densities as high as 30 or $100 \mathrm{~mA} / \mathrm{cm}^{2}$, though the fact that significant arsenic was removed at these current densities through ECAR suggests that passivation in this region did not occur. It is worthwhile to note that the $\mathrm{pH}$ of the solution during the scan was likely as high as $\mathrm{pH}=9.14$ (see Section 3.2 .9 ), and the passivation potential is known to shift with $\mathrm{pH}$. However, the passivation potential shifts cathodically (lower in voltage) with increasing $\mathrm{pH}$, making it more likely that passivation would be seen in this system than in the real system at neutral $\mathrm{pH}$.

The lack of passivation up to current density $10 \mathrm{~mA} / \mathrm{cm}^{2}$ is significant. Mameri et al. (1998) point out that all teams working with aluminum monopolar electrodes to remove fluoride via electrocoagulation met with passivation problems. The lack of passivation in iron over the current density region that is most effective for arsenic removal is promising in terms of maintenance requirements and the ability to operate in monopolar mode.

\footnotetext{
${ }^{13}$ Note that higher current densities may also have energy consumption disadvantages if a higher operating current is used (as opposed to decreasing the active electrode area), requiring a higher voltage.
} 
It is also significant to see that the potential at current densities as high as 10 $\mathrm{mA} / \mathrm{cm}^{2}$ is well below the potential required for oxygen evolution, which occurs near $0.8 \mathrm{~V}$ vs $\mathrm{SHE}$ at atmospheric pressure and $\mathrm{pH}=7$, and near $0.7 \mathrm{~V}$ vs $\mathrm{SHE}$ near $\mathrm{pH}=$ 9 (Misawa, 1973) . Oxygen evolution can compete with iron dissolution for electrons, reducing the current efficiency (Equation 2.21). This leads to fewer iron (hydr)oxides produced per coulomb and hence less arsenic removal capacity. Recall that one possible explanation for the decrease in arsenic removal capacity seen at $j>5.0 \mathrm{~mA} / \mathrm{cm}^{2}$ was the onset of oxygen evolution. While the data in Figure 3.22 cannot rule out oxygen evolution at $j>30 \mathrm{~mA} / \mathrm{cm}^{2}$, it makes this explanation highly unlikely for $j \leq 10.0 \mathrm{~mA} / \mathrm{cm}^{2}$. The onset of oxygen evolution also limits the highest current density that could potentially be effective in ECAR. Because that limit has not yet been reached, higher current densities should be explored to look for possible increases in arsenic removal capacity (if the composition of EGA changes favorably) that may occur before reaching the oxygen evolution potential. Since higher current densities can deliver more charge in less time, this could potentially provide a very favorable parameter space for arsenic removal.

Thermodynamic equilibrium potentials at $25^{\circ} \mathrm{C}$ for appropriate redox couples are shown in Figure 3.22 for $\mathrm{pH}=7$ (in red) - expected of natural groundwater - and $\mathrm{pH}$ $=9$ (in blue) - close to the final $\mathrm{pH}$ of the scan. A Pourbaix diagram composed by Bang et al. (2005a) assuming solid $\mathrm{Fe}, \mathrm{Fe}(\mathrm{OH})_{2}$ and $\mathrm{Fe}(\mathrm{OH})_{3}$ exist was consulted to select appropriate lines at each pH. If concentration appears in the equilibrium calculation, a range of concentrations from $10^{-8} \mathrm{M}-1 \mathrm{M}$ are shown. It is difficult to make direct comparisons to ECAR treatment due to the high $\mathrm{pH}$ of the scan, however some general 
comparisons are useful to suggest further testing.

Current densities $j=0.02 \mathrm{~mA} / \mathrm{cm}^{2}$ and $j=0.07 \mathrm{~mA} / \mathrm{cm}^{2}$ both occur to the anodic side of the transition between $\mathrm{Fe}(0)$ and $\mathrm{Fe}[\mathrm{II}]$ species and to the cathodic side of the transition between $\mathrm{Fe}[\mathrm{II}]$ to Fe[III] species for both $\mathrm{pH} 7$ and 9. This suggests that the iron (hydr)oxides produced at these current densities are based predominantly on Fe[II] species rather than $\mathrm{Fe}[\mathrm{III}]$, though there is some uncertainty due to the high $\mathrm{pH}$ during the scan $(\mathrm{pH}=9.14)$ relative to natural groundwater. Higher current densities fall to the anodic side of the transition between $\mathrm{Fe}[\mathrm{II}]$ and Fe[III] species, making it more likely that the iron (hydr)oxides are composed of Fe[III]. The increased arsenic removal capacity seen at current densities $j=0.02$ and $0.07 \mathrm{~mA} / \mathrm{cm}^{2}$ suggests that $\mathrm{Fe}[\mathrm{II}]$ species may have higher sorption capacities than Fe[III] species. Alternatively, Fe[III](hydr)oxides formed from the oxidation of Fe[II] may have a higher adsorption capacity for arsenic than Fe[III](hydr)oxides formed from Fe[III] directly. Roberts et al. (2004) found that both $\mathrm{As}[\mathrm{III}]$ and $\mathrm{As}[\mathrm{V}]$ removal efficiencies were higher with the chemical addition of Fe[II] salts (quickly oxidized to Fe[III]) than the addition of Fe[III] salts in simulated groundwater containing phosphate and silicate. This suggests that Fe[III](hydr)oxides produced from $\mathrm{Fe}[\mathrm{II}]$ oxidation may have higher adsorption capacities than $\mathrm{Fe}[\mathrm{III}]$ in Bangladesh groundwater.

Interestingly, the equilibrium potential for $\mathrm{As}[\mathrm{III}]$ oxidation to $\mathrm{As}[\mathrm{V}]\left(\mathrm{H}_{3} \mathrm{AsO}_{3} / \mathrm{HAsO}_{4}^{2-}\right.$ or $\mathrm{H}_{2} \mathrm{AsO}_{4}^{-}$) is above the potential associated with current densities $j<0.7 \mathrm{~mA} / \mathrm{cm}^{2}$ for $\mathrm{pH} 7$ and $j<5.0 \mathrm{~mA} / \mathrm{cm}^{2}$ for $\mathrm{pH} 9$. This means that it is thermodynamically favorable for $\mathrm{As}[\mathrm{V}]$ to reduce to $\mathrm{As}[\mathrm{III}]$ at many current densities. Matrix 1 and Matrix 2 results 
indicate that As[III] is effectively removed at all current densities tested - previously attributed to As[III] oxidation followed by As[V] complexation to iron (hydr)oxides. These results indicate the possibility that As[III] is effectively removed due to direct complexation with iron (hydr)oxides rather than oxidation followed by complexation. Due to kinetics and other effects, equilibrium potentials do not prove that $\mathrm{As}[\mathrm{V}]$ is being reduced at low current densities, but it does suggest that further testing would be appropriate. Note also that direct complexation of As[III] would be consistent with the results showing significant As[III] removal using post-synthesis ECAR-generated adsorbent.

\subsubsection{Sedimentation versus $0.1 \mu \mathrm{m}$ vacuum filtration}

The arsenic removal capability of ECAR using $0.1 \mu \mathrm{m}$ filtration was compared to that of ECAR using sedimentation (i.e. quiescent settling followed by decantation) during both the Matrix 1 and Matrix 2 experiments. Figure 3.23 shows the the final arsenic concentration of four Matrix 1 experiments utilizing $0.1 \mu \mathrm{m}$ filtration compared to the experiments of the same operating conditions utilizing sedimentation. The four experiments cover a range of settling times, $T s$, before decantation $(T s=1.9-6$ days $)$ and represent experiments from a range of operating conditions $\left(j=0.07-1.1 \mathrm{~mA} / \mathrm{cm}^{2}\right.$, $q=15-85 \mathrm{C} / \mathrm{L}, t_{C P}=3-50 \mathrm{~min}$; see Figure 3.23 caption for exact conditions of each). For the first three experiments (1-3), with $T s \leq 3.1$ days, the arsenic concentration in settled samples is much higher (by $10-64 \mu \mathrm{g} / \mathrm{L}$ ), that the $0.1 \mu \mathrm{m}$ filtered samples. In one case (experiment 2), the final arsenic concentration of the $0.1 \mu \mathrm{m}$ filtered sample is lower than the WHO limit of $10 \mu \mathrm{g} / \mathrm{L}$, while the sedimentation sample ( $T s=1.9$ 




Figure 3.23: Comparison of the final arsenic concentration for $0.1 \mu \mathrm{m}$ filtered and sedimentation samples from the Matrix 1 series of experiments. Ts, listed below each series of bars, is the quiescent settling time allowed before decantation for the settled sample $(0.1 \mu \mathrm{m}$ filtered samples were all filtered immediately after mixing). In the case of series 4 (right), a second sample was removed and tested from the sedimentation beaker at a later time. The ECAR operating conditions for the above experiments were (1) $j=1.1$ $\mathrm{mA} / \mathrm{cm}^{2}, q=23 \mathrm{C} / \mathrm{L}, t_{C P}=3 \mathrm{~min} ;(2) j=1.1 \mathrm{~mA} / \mathrm{cm}^{2}, q=85 \mathrm{C} / \mathrm{L}, t_{C P}=5 \mathrm{~min}$; (3) $j$ $=0.07 \mathrm{~mA} / \mathrm{cm}^{2}, q=25 \mathrm{C} / \mathrm{L}, t_{C P}=50 \mathrm{~min}$; and $(4) j=0.70 \mathrm{~mA} / \mathrm{cm}^{2}, q=15 \mathrm{C} / \mathrm{L}, t_{C P}$ $=3$ min. Each series has been normalized to its average initial arsenic concentration to avoid small deviations due to different starting arsenic levels - the average initial arsenic concentration was $610 \pm 40 \mu \mathrm{g} / \mathrm{L}$. 


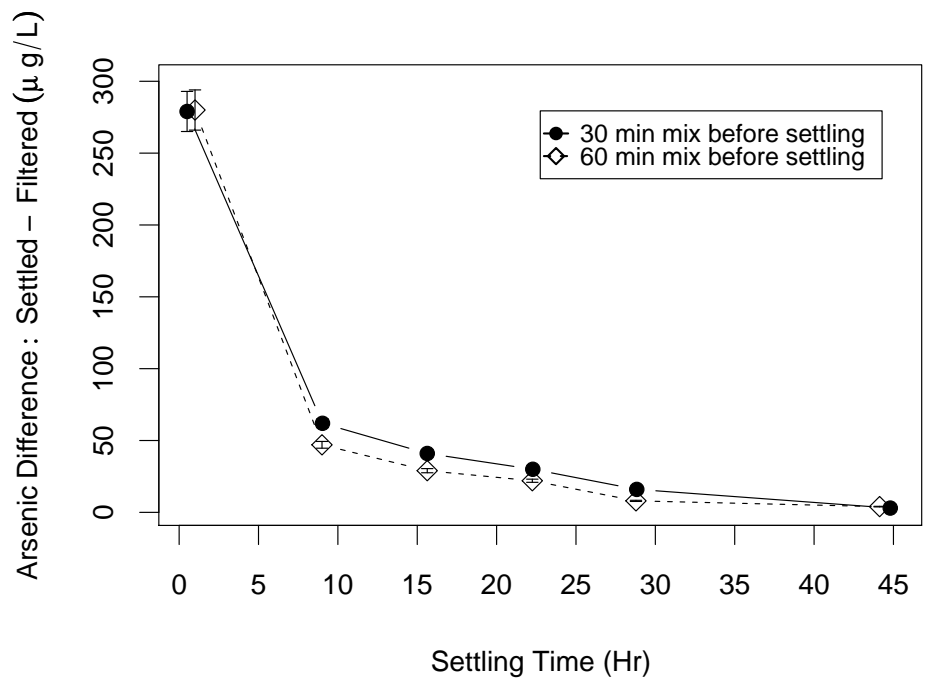

Figure 3.24: The difference in arsenic concentration between samples filtered through a $0.1 \mu \mathrm{m}$ membrane and samples decanted from the top of a beaker after ECAR treatment $\left(j=1.1 . \mathrm{mA} / \mathrm{cm}^{2}, q=100 \mathrm{C} / \mathrm{L}\right)$ following a period of quiescent settling as a function of the quiescent settling time. Two tests are shown in which the solution was mixed for 30 minutes before quiescent settling (solid line, solid symbols) and mixed for 60 minutes before quiescent settling (dashed line, open symbols). Lines have been added to guide the eye only. 
days) is higher than even the Bangladesh limit of $50 \mu \mathrm{g} / \mathrm{L}$. All of this indicates that 3.1 days is too short for sedimentation to be as effective or nearly as effective as $0.1 \mu \mathrm{m}$ filtration, specifically with respect to reducing levels to below the WHO limit. For the final experiment (4), the first sedimentation sample was decanted after settling for 3.8 days. This sample shows a lower arsenic concentration (by about $20 \mathrm{ppb}$ ) than the immediately filtered sample, probably due to the additional complexation time allowed by settling and/or increase oxidation of $\mathrm{As}[\mathrm{III}]$ into $\mathrm{As}[\mathrm{V}]$ due to atmospheric exposure over time. An additional observation after 6 days shows the same arsenic concentration as found after 3.8 days, indicating that the concentration has stabilized some time before 3.8 days. Thus arsenic removal from SBGW-1 water using ECAR with sedimentation can be as good as or even better than (due to the increased complexation time) ECAR with $0.1 \mu \mathrm{m}$ filtering, as long as the sample is allowed to settle for at least 3.8 days (or possibly some time between $3.1-3.8$ days).

At one point, salvaged hard drive magnets were attached to the bottom of a beaker containing dosed and mixed SBGW-1 water (Matrix 1 procedure) and the settling rate compared to a beaker containing the same effluent with no magnets. After 21 hours of settling, both beakers appeared clear on top with an orange precipitate uniformly spread over the bottom of the beaker. There was a very slight outline of the magnets made by precipitate, indicating that the ECAR-generated particles are only slightly magnetic. QuickTest measurements of the supernatant showed the same arsenic concentration within error $(50 \pm 25 \mu \mathrm{g} / \mathrm{L})$ for magnet-enhanced and non-magnetenhanced settling. A repeat of this test yielded the same results after 3.5 hours of settling, 
though in this case, no visible outline of the magnet was observed in the precipitate. It is likely that the ECAR-generated particles in this current density range $(0.07-1.1$ $\mathrm{mA} / \mathrm{cm}^{2}$ ) are not magnetic enough for settling to be aided by low-cost magnets.

Using the Matrix 2 procedure and SBGW-2 water, sedimentation has been compared to $0.1 \mu \mathrm{m}$ filtration over a period of two days by sampling the top effluent of a quiescent sample after ECAR treatment (at $j=1.1 \mathrm{~mA} / \mathrm{cm}^{2}, q=100 \mathrm{C} / \mathrm{L}, t_{C P}=44.7$ min) and comparing the unfiltered effluent to the filtered effluent. Two sedimentation samples were compared in order to see if sedimentation could be accelerated by initial mixing, including one experiment with dosed water that was mixed for 30 minutes before settling and another that was mixed for 60 minutes before settling. The results of both are shown in Figure 3.24.

From Figure 3.24 it is clear that sedimentation could not replace $0.1 \mu \mathrm{m}$ vacuum filtration immediately after mixing, at which time the sedimentation sample contains $275 \mu \mathrm{g} / \mathrm{L}$ more arsenic than the $0.1 \mu \mathrm{m}$ filtered sample. In fact, the difference in arsenic concentration does not approach zero until nearly 45 hours, or 1.9 days, have passed (the final difference in arsenic measured was $3 \mu \mathrm{g} / \mathrm{L}$ in both experiments). This implies that a two-day storage time would be required to replace $0.1 \mu \mathrm{m}$ filtration with sedimentation to yield the same results.

The sample that had been mixed for 60 minutes prior to settling did settle slightly faster than the sample that had been mixed for 30 minutes, though the gap began to close with time. Both sedimentation samples were $3 \mu \mathrm{g} / \mathrm{L}$ above the $0.1 \mu \mathrm{m}$ filtered samples after 45 hours. This is consistent with a slowing of the settling rate of the 
60-min sample relative to the 30 -min sample over time. It is also consistent with reaching a maximum level of removal via sedimentation. For example some particles may be too small to settle but large enough to be removed on a $0.1 \mu \mathrm{m}$ membrane. In this case, the 60-min sample may have reached this limit anytime between 28 and 45 hours. Thus it is possible that the additional mixing could reduce the required settling time by up to about 10 hours, making the total required settling time as low as 1.5 days. Additional testing with more frequent samples between 28 and 45 hours and longer mixing times could determine if increasing the mixing time further does in fact reduce the settling time, and if so, if there is a mixing time such that the settling time could be reduced substantially.

\subsection{Chapter Summary}

Two bench-top electrochemical cells have been designed and incorporated into a small-scale ECAR batch treatment procedure. Two recipes for synthetic Bangladesh groundwater (SBGW-1 and SBGW-2) have been developed, differing in the levels of competitive co-occuring solutes phosphate, silicate, bicarbonate, and sulfate. The resistivity of SBGW-1 was measured to be $\rho_{e}=355 \Omega \cdot \mathrm{cm}$ using impedance spectroscopy (EIS). This high resistivity will likely lead to a high voltage requirement in real Bangladesh groundwater, potentially mitigated by the addition of a small amount of salt before treatment.

The arsenic removal capability of ECAR in synthetic Bangladesh groundwater $(\mathrm{SBGW})$ at $\mathrm{pH}=7$ was demonstrated in two distinct groundwater recipes (SBGW-1 and 
SBGW-2, composition in Table 3.1) by reducing initial concentrations of 550-580 $\mu \mathrm{g} / \mathrm{L}$ arsenic (including both $\mathrm{As}[\mathrm{III}]$ and $\mathrm{As}[\mathrm{V}]$ in ratio $\mathrm{As}[\mathrm{III}]: \mathrm{As}[\mathrm{V}]=1: 1$ ) to below the WHO limit of $10 \mu \mathrm{g} / \mathrm{L}$. This was explicitly demonstrated for current densities $j=0.30$ - $1.1 \mathrm{~mA} / \mathrm{cm}^{2}$ in SBGW-1 and $j=0.02-5.0 \mathrm{~mA} / \mathrm{cm}^{2}$ in SBGW-2. Based on trends, it is likely that the WHO limit would be reached for $j=0.07 \mathrm{~mA} / \mathrm{cm}^{2}$ in SBGW-1 and $j=10,30$, and $100 \mathrm{~mA} / \mathrm{cm}^{2}$ in SBGW-2 with a small increase in charge density. The legal Bangladesh limit $(50 \mu \mathrm{g} / \mathrm{L})$ was explicitly reached in every case. Arsenic removal capability in SBGW-2 is better indicator of removal capability in Bangladesh than SBGW-1 due to the average levels co-occurring solutes known to compete with arsenic for adsorption, such as phosphate and silicate.

The charge density required to reach the Bangladesh and WHO limit in batch tests (extrapolated in cases where it was not explicitly reached) is listed in Table 3.5. Values based on the WHO limit were used to calculate arsenic removal capacity, iron capacity (assuming dissolution of $\mathrm{Fe}^{3+}$ ), and total treatment time. The charge density required to reach the WHO limit varied from $q_{W H O}=25-70 \mathrm{C} / \mathrm{L}$ in SBGW-1 and $q_{W H O}=25-180 \mathrm{C} / \mathrm{L}$ in SBGW-2 depending on current density. The corresponding arsenic removal capacity was $8-22 \mu \mathrm{g}-\mathrm{As}_{\mathrm{rem}} / \mathrm{C}$ in SBGW-1 and $3-22 \mu \mathrm{g}-\mathrm{As}_{\mathrm{rem}} / \mathrm{C}$ in SBGW-2.

Arsenic removal capacity was found to be approximately constant within certain ranges of current density, and highly variable between ranges. In order of decreasing removal capacity, the pattern was: $0.02 \mathrm{~mA} / \mathrm{cm}^{2}>0.07 \mathrm{~mA} / \mathrm{cm}^{2}>0.30-1.1 \mathrm{~mA} / \mathrm{cm}^{2}$ $>5.0-100 \mathrm{~mA} / \mathrm{cm}^{2}$. These results are consistent with both Kumar et al. (2004) and 
Gomes et al. (2007), who found no effect of current density on arsenic removal efficiency within a range of $j=0.64-1.53 \mathrm{~mA} / \mathrm{cm}^{2}$ and $j=3-30 \mathrm{~mA} / \mathrm{cm}^{2}$ respectively. Both of these ranges fall within the current density pockets of stable arsenic removal capacity.

Batch test runs Matrix 1 and Matrix 2 (described in Sections 3.2.6 and 3.2.7) used charge density, $q$, and current density, $j$, as independent variables. The current processing time, $t_{C P}$ (which is a function of $q, j$, and the electrode surface area to volume ratio, A/V according to Equation 2.34), was allowed to vary over an order of magnitude between tests at $1.1 \mathrm{~mA} / \mathrm{cm}^{2}$ and $0.02 \mathrm{~mA} / \mathrm{cm}^{2}$. Surprisingly, a 6-fold increase in removal capacity was observed at $0.02 \mathrm{~mA} / \mathrm{cm}^{2}$ compared to $1.1 \mathrm{~mA} / \mathrm{cm}^{2}$. In order to determine if this increase was due to $j$ alone or the large difference in $t_{C P}$, an additional batch test was performed at $1.1 \mathrm{~mA} / \mathrm{cm}^{2}$ using the same $t_{C P}$ as the $0.02 \mathrm{~mA} / \mathrm{cm}^{2}$ batch test. This revealed that most, but not all, of the increase in removal capacity originally seen at $0.02 \mathrm{~mA} / \mathrm{cm}^{2}$ was due to the increase in $t_{C P}$. Comparisons of the two batch tests at $1.1 \mathrm{~mA} / \mathrm{cm}^{2}$ showed that removal capacity could be increased 3 -fold at the cost of increasing $t_{C P}$ from $\sim 1$ hour to $\sim 19$ hours. This finding indicates that increasing $t_{C P}$ can increase arsenic removal capacity independently of $q$. This effect could be due to the increase in contact time between EGA, the increase in duration of As[III] oxidation to $\mathrm{As}[\mathrm{V}]$, increased duration of coagulation in the presence of an electric field, or some unknown effect. If it is due to the increase in contact time, then adjusting the mixing time, $t_{M}$ should have the same effect as increasing $t_{C P}$. Further testing is needed to determine if $t_{C P}$ should be considered an additional controlling parameter (in addition to $j, q$, and $t_{M}$ ) and to determine if small increases in $t_{C P}$ (such that total treatment 
time is constrained to 6-7 hours) are capable of increasing arsenic removal capacity in a significant way.

Batch tests at $j=1.1 \mathrm{~mA} / \mathrm{cm}^{2}$ were performed in both SBGW-1 (containing low phosphate, average bicarbonate, and no silicate) and SBGW-2 (containing average levels of phosphate, bicarbonate, and silicate for Bangladesh groundwater). The additional phosphate and silicate in SBGW-2 lead to a 54\% decrease in arsenic removal capacity relative to the WHO limit. This is consistent with previous studies of by Roberts et al. (2004) of competitive adsorption between arsenic and phosphate or arsenic and silicate during coprecipitation with Fe[III] salts.

No single set of operating parameters was optimal in terms of both arsenic removal capacity and treatment time. Based on batch tests, two sets of parameters with different advantages were chosen. The parameter set with the highest overall arsenic removal capacity(i.e. the lowest operating costs) was $j=0.02 \mathrm{~mA} / \mathrm{cm}^{2}, q=25 \mathrm{C} / \mathrm{L}$, $t_{C P}=416 \min (\sim 7 \mathrm{hrs}), t_{M}=60 \mathrm{~min}$. Total treatment time $(8 \mathrm{hrs})$ was just above the target for a community water system (6-7 hrs). Constraining the treatment time to be around 2 hours, the highest arsenic removal capacity was achieved with $j=1.1 \mathrm{~mA} / \mathrm{cm}^{2}$, $q=150 \mathrm{C} / \mathrm{L}, t_{C P}=69 \mathrm{~min}, t_{M}=60 \mathrm{~min}$. Both of these parameter sets have advantages (the first in terms of operating costs, and the second in terms of treatment time) and choosing between them would require more detailed knowledge on the importance of reduced operating costs compared to treatment time.

In order to explore the significance of producing iron (hydr)oxides in-situ with arsenic during ECAR and study adsorbent aging, experiments were performed using 
iron (hydr)oxides that were not generated in-situ. Post-synthesis ECAR-generated iron (hydr)oxides adsorbent, or PS-EGA adsorbent, was created by running the ECAR dosing process in synthetic Bangladesh groundwater containing no arsenic. Freshly made PSEGA was able to reduce initial concentrations of $\sim 600 \mu \mathrm{g} / \mathrm{L}$ (including As[III] and As $[\mathrm{V}]$ in a 1:1 ratio) to $37 \mu \mathrm{g} / \mathrm{L}$ after 60 minutes of contact and $22 \mu \mathrm{g} / \mathrm{L}$ after 48 hours of contact. ECAR treatment under comparable conditions was able to reduce arsenic to $10 \mu \mathrm{g} / \mathrm{L}$ after 60 minutes of mixing. The low arsenic concentration implies that most of the initial $300 \mu \mathrm{g} / \mathrm{L}$ of As[III] was removed with PS-EGA without electrochemical oxidation. This As[III] could be forming complexes with PS-EGA directly, or being oxidized in parallel with $\mathrm{Fe}[\mathrm{II}]$ oxidation by dissolved $\mathrm{O}_{2}$. Significantly however, the adsorbent was not able to reduce total arsenic levels to below the WHO limit $(10 \mu \mathrm{g} / \mathrm{L})$, indicating that the in-situ generation of EGA during ECAR is significant to its arsenic removal capability.

Adsorption onto freshly prepared PS-EGA was compared to adsorption onto PS-EGA allowed to age for 60 minutes after being generated (aged-PS-EGA). Removal capacity (as $\mu \mathrm{g}-\mathrm{As}_{\mathrm{rem}} / \mathrm{mg}_{\mathrm{F}} \mathrm{Fe}^{3+}$ ) was $33 \%$ lower for aged-PS-EGA than fresh-PS-EGA after 60 minutes of contact and $13 \%$ lower after 48 hours $\left(\operatorname{As}_{\text {final }}=100 \mu \mathrm{g} / \mathrm{L}\right)$. This indicates that removal capacity of EGA is highly sensitive to aging. This is likely due to the formation of aggregates. Once aggregates form, arsenic oxyanions must diffuse into them to find available sites. It is possible that the adsorption capacity of agedPS-EGA could catch up with that of fresh-PS-EGA given enough time for diffusion into aggregates to occur. However, given the constraints on treatment time proposed in 
Chapter 1, attempting to form PS-EGA at a central location and shipping the adsorbent to satellite treatment sites in place of on-site ECAR treatment is not advised.

Electrochemical oxidation ${ }^{14}$ of $\mathrm{As}[\mathrm{III}]$ to $\mathrm{As}[\mathrm{V}]$ and subsequent adsorption of As $[\mathrm{V}]$ onto EGA has been proposed as the mechanism of effective As[III] removal in electrocoagulation. Measurements of As[III] concentration during ECAR treatment indicate that the arsenic removal capacity of ECAR for As[III] is approximately 3 times lower than the removal capacity for As[V]. This is consistent both with (1) EGA having a lower affinity for As[III] (thereby requiring a higher dosage) or (2) slow oxidation of As[III], requiring more current processing time rather than more charge. Freshly made PS-EGA (see previous paragraphs) was able to remove $90 \%$ of added As[III] after 60 minutes of contact in SBGW containing competitive ions such as phosphate, silicate, bicarbonate, and As $[\mathrm{V}]$. No electrodes were present or external voltage applied during the contact time, preventing electrochemical oxidation of As[III]. This implies that either (a) EGA has a significant affinity for As[III] on the timescale of ECAR treatment, even in the presence of competing ions, or (b) As[III] is being oxidized by something other than the anode, perhaps in parallel with $\mathrm{Fe}[\mathrm{II}]$ oxidation due to dissolved $\mathrm{O}_{2}$. Final As[III] concentrations are still lower after ECAR treatment than contact with PS-EGA, implying that either electrochemical oxidation is significant in reducing low arsenic concentrations down to less than $10 \mu \mathrm{g} / \mathrm{L}$, or perhaps that EGA generated in-situ with As[III] has a higher arsenic removal capacity than PS-EGA.

Transient voltammetry was performed on the iron electrode at $\nu=0.1 \mathrm{mV} / \mathrm{s}$ in

\footnotetext{
${ }^{14}$ Technically, all redox reactions are electrochemical, however, here I am referring to electrochemical oxidation caused by the external voltage applied.
} 
$0.1 \mathrm{M} \mathrm{KClO}_{4}$ (plain perchlorate) solution and $0.1 \mathrm{M} \mathrm{KClO}_{4}$ plus synthetic Bangladesh groundwater (SBGW-1 recipe). A comparison of the resulting oxidation scans at low potentials revealed an anodic shift of approximately $50 \mathrm{mV}$ in SBGW-1 compared to plain perchlorate solution, resulting in a large loss of current. This suggests that species in the SBGW interfere with the dissolution processes on the iron electrode. This resulting increase in the overpotential required to reach a given current density in SBGW will add to the energy consumption of ECAR treatment. Since the overpotential is less severe at higher current densities, this could give high current densities some advantage over low current densities in terms of energy consumption. This could help offset disadvantages due to higher operating current.

Electrode passivation, a common problem during electrocoagulation with aluminum electrodes, is not seen within the range of the scan (up to current density $j=$ $\left.10 \mathrm{~mA} / \mathrm{cm}^{2}\right)$. The lack of passivation in iron over the current density region that is most effective for arsenic removal is promising in terms of electrode longevity, maintenance requirements, and the ability to operate in monopolar mode.

The polarization scan revealed that the electrode potential over the entire range of scanned current density (i.e. $j \leq 10 \mathrm{~mA} / \mathrm{cm}^{2}$ ) is well below the thermodynamic equilibrium potential for oxygen evolution. This rules out oxygen evolution as a cause of the decrease in arsenic removal capacity seen at higher current density. The onset of oxygen evolution also limits the highest current density that could potentially be effective in ECAR. Because that limit has not yet been reached, higher current densities should be explored to look for possible increases in arsenic removal capacity (if the composition of 
EGA changes favorably) that may occur before reaching the oxygen evolution potential. Since higher current densities can deliver more charge in less time, this could potentially provide a very favorable parameter space for arsenic removal.

A comparison of the polarization scan in SBGW to thermodynamic equilibrium potentials for relevant iron species revealed that the lowest current density $(j=$ $0.02 \mathrm{~mA} / \mathrm{cm}^{2}$ ) is likely to produce iron (hydr)oxides based on Fe[II], while the higher current densities are likely to produce iron (hydr)oxides based on Fe[III]. The high removal capacity seen at low current density suggests that Fe[II] species may have a higher affinity for arsenic than Fe[III] species. A comparison of the polarization scan in SBGW to thermodynamic equilibrium potentials for relevant arsenic species revealed that As[V] reduction to As[III] may be thermodynamically favorable at the lower current densities tested. The fact that arsenic removal capacity is higher at low current density suggests that electrochemical oxidation of As[III] may not be essential for effective and efficient As[III] removal in ECAR. This is consistent with the relatively high adsorption of As[III] achieved by post-synthesis EGA after 60 minutes of contact in SBGW-2.

The arsenic removal capability of ECAR using $0.1 \mu \mathrm{m}$ filtration was compared to that of ECAR using sedimentation (i.e. quiescent settling followed by decantation). Dosed and mixed synthetic Bangladesh groundwater samples (SBGW-1) were left to settle over a period 2 - 6 days and compared to samples filtered immediately after mixing. For a settling time of less than 3.1 days, sedimentation samples contained $10-64 \mu \mathrm{g} / \mathrm{L}$ more arsenic than $0.1 \mu \mathrm{m}$ filtered samples. After 3.8 days of settling, sedimentation samples contained approximately $20 \mu \mathrm{g} / \mathrm{L}$ less arsenic than $0.1 \mu \mathrm{m}$ filtered samples. The 
increase in arsenic removed is probably due to the additional complexation time allowed by the settling period. Repeated sampling after 6 days of settling showed no additional decrease. Attempts to increase the settling rate using magnets were unsuccessful, suggesting that the EGA sludge is not very magnetic. Sedimentation tests using SBGW-2 water produced similar results. The difference in arsenic concentration between sedimentation samples and $0.1 \mu \mathrm{m}$ filtered samples approached zero after 1.9 days of settling. Increasing the mixing time by 30 minutes before quiescent settling accelerated the initial stages of settling, but 1.9 days was still required before sedimentation results began to approach $0.1 \mu \mathrm{m}$ filtered results. These tests imply that sedimentation cannot replace $0.1 \mu \mathrm{m}$ filtration without requiring water storage for up to 2 days or the addition of a coagulant. 


\section{Chapter 4}

\section{Characterization of Reaction}

\section{Products}

\subsection{Introduction}

Electrochemical arsenic remediation (ECAR) produces arsenic-laden iron (hydr)oxide sludge (EGA) during the treatment process. EGA sludge is composed of the reaction products formed during electrolysis (also called coulombic dosing) and post-electrolysis mixing ${ }^{1}$. Characterization of these reaction products is important to (1) characterize the particles that must be separated from potable water in the filtration step, (2) characterize the waste byproduct associated with ECAR treatment, (3) explore the reactions and arsenic removal mechanism in ECAR, and (4) explore the effect of ECAR operating parameters on arsenic removal. Each will be described in turn.

\footnotetext{
${ }^{1}$ For a review of the three stages in ECAR treatment, see Section 3.2.5.
} 
ECAR treatment involves a coulombic dosing stage, in which EGA particles are generated in arsenic-contaminated solution, a mixing stage, in which complexation between arsenic and EGA along with coagulation occurs, and a separation or filtration stage, in which arsenic-laden EGA is separated from potable water. In laboratory scale batch tests, filtration was achieved using $0.1 \mu \mathrm{m}$ pore size membrane filters (see Chapter 3). However, in rural Bangladesh, a low-cost alternative is desirable. Previous experiments to separate particles through simple settling and decantation (Chapter 3) revealed that $>3$ days of settling is required to achieve the same level of particle separation as $0.1 \mu \mathrm{m}$ filtration. A more detailed study of alternative low-cost options is needed, for which it is useful to characterize the average EGA particle size, shape, and homogeneity. Scanning electron microscopy (SEM) is a technique capable of imaging surface topography with high resolution $(\sim 5 \mathrm{~nm})$. SEM images can be used to estimate the primary aggregate size, particle shape, and homogeneity of an EGA sample. A simple comparison of filtrate arsenic concentration after membrane filtration with different pore sizes can help constrain the cluster size of EGA as well.

Arsenic-laden EGA sludge must be disposed of as a waste product after ECAR treatment. The amount and chemical composition of the waste produced are key to understanding safe disposal mechanisms and potential disposal costs in Bangladesh. Chemical analysis of dissolved EGA sludge can provide information about the arsenic content as well as the As/Fe ratio of the waste. The total sludge produced after ECAR treatment of synthetic Bangladesh groundwater can be weighed to estimate the waste per coulomb expected in Bangladesh. The strength and stability of the surface complexes between 
arsenic and EGA are also relevant to safe waste disposal. X-ray absorption fine structure (EXAFS) spectroscopy has been widely used to study the bonding mechanism of arsenic by geosorbents, such as iron (hydr)oxides (Fendorf et al., 1997; Paktunc et al., 2003). Inner sphere complexation between As and Fe hydroxides has been shown by EXAFS (Waychunas et al., 1993; Manceau, 1995; O’Reilly et al., 2001). EXAFS also showed that bidentate binuclear complexation was the major bonding mechanism for arsenate adsorption onto goethite (Fendorf et al., 1997; Waychunas et al., 1993; Manceau, 1995; O'Reilly et al., 2001). If a similar type of strong bonding exists between arsenic and EGA, this could provide initial evidence that As would remain immobilized in EGA waste.

An understanding of the arsenic bonding structure to EGA is also relevant to understanding the dominant arsenic removal mechanism in ECAR. EXAFS spectroscopy can distinguish between surface complexes of arsenic onto EGA and the formation of a solid phase, such as $\mathrm{FeAsO}_{4}$ (scorodite) (Foster, 2003). The primary location of arsenic removal (i.e. on the surface layer of the electrode versus within the bulk solution) could also be determined using EXAFS spectroscopy. Iron EXAFS spectroscopy and X-ray absorption near edge spectroscopy (XANES) could also be used to characterize the dominant iron (hydr)oxides structure of EGA. Fe atoms are 6-fold coordinated in iron oxyhydroxide (Manceau \& Combes, 1988). The 3D chains are made up of single or multiple chains of edge sharing octahedra that in turn may be joined along their lengths by sharing faces (e.g. Hematite), edges (e.g. lepidocrocite), or corners (goethite). Fe-Fe distances are roughly characteristic of a given polyhedral linkage (Manceau \& Combes, 
1988). Fe-XANES and EXAFS has been used to determine the oxidation state of iron compounds and distinguish between mineral classes such as oxides and oxyhydroxides (O’Day et al., 1994).

In Chapter 3, ECAR batch tests in synthetic Bangladesh groundwater revealed some difference in arsenic removal capacity with current density. Polarization curves ruled out oxygen evolution as the primary cause. It was suggested that these differences in removal capacity could be due to differences in EGA composition at different current densities. It was further suggested by polarization curves and thermodynamic equilibrium lines that EGA generated at lower current densities (with higher removal capacity) might be based on Fe[II] species while EGA generated at higher current densities might be based on Fe[III]-species. Fe XANES spectroscopy could determine the primary oxidation state of Fe in EGA. Fe EXAFS spectroscopy could be used to compare the EGA structure generated at different current densities and look for differences. As EXAFS spectroscopy is sensitive to the bonding structure of the iron compounds preferred by arsenic. As EXAFS of EGA generated at different current densities could reveal if arsenic prefers bonding to different components of EGA even if the primary component of EGA is unchanging.

\subsubsection{Research objectives}

The primary goal of this research was to characterize the EGA reaction products of ECAR generated at different current densities using chemical analysis, SEM, XANES, and EXAFS spectroscopy. The purpose of this characterization was fourfold: (1) to 
physically characterize the particles that must be separated from potable water in the filtration step of ECAR, (2) to characterize the waste products associated with ECAR treatment for safe disposal, (3) to determine the bonding structure of arsenic complexed to EGA responsible for arsenic removal in ECAR, and (4) to explore a difference in EGA composition as the primary cause of arsenic removal capacity difference seen at different current densities.

\section{Specific Research Objectives}

1. Characterize the primary aggregate size, cluster size, particle shape, and homogeneity of EGA for the purpose of designing a low-cost filtration mechanism in Bangladesh.

2. Measure the As content and $\mathrm{Fe} / \mathrm{As}$ ratio of arsenic-laden waste produced in ECAR at various current densities and use this to estimate the amount of waste per coulomb generated in ECAR.

3. Compare the iron (hydr)oxides structure of EGA produced at different current densities (EXAFS).

4. Compare the arsenic-EGA bonding structure in arsenic-laden EGA at different current densities (EXAFS).

5. Compare the iron (hydr)oxides structure of EGA to known reference iron oxyhydroxide and iron hydroxide compounds (EXAFS).

6. Compare the arsenic-iron (hydr)oxides bonding structure in arsenic-laden EGA to 
the known bonding structure of arsenic adsorbed to various iron (hydr)oxides and scorodite (EXAFS).

\subsection{Methods}

\subsubsection{Arsenic breakthrough as a function of membrane pore size}

A rough limit on the cluster size of EGA particles was measured during the filtration stage of ECAR using membrane filters with progressively larger pore sizes, from $0.1-0.8 \mu \mathrm{m}$. The membranes used were Millipore polyvinylidene fluoride hydrophilic ( $0.1 \mu \mathrm{m})$, Pall Supor $450(0.45 \mu \mathrm{m})$ and Pall supor $800(0.8 \mu \mathrm{m})$. In each case the pore size is the measure of the largest pore ${ }^{2}$. The sample used was SBGW-1 recipe synthetic Bangladesh groundwater treated at $j=0.70 \mathrm{~mA} / \mathrm{cm}^{2}, q=14.8 \mathrm{C} / \mathrm{L}, t_{C P}=3 \mathrm{~min}$, $t_{M}=60-135 \mathrm{~min}$. The arsenic concentration (measured via Quick Test described in Section 3.2.1) in $0.1 \mu \mathrm{m}$ pore size filtrate was stable (within $5 \mu \mathrm{g} / \mathrm{L}$ ) between $t_{M}=40 \mathrm{~min}$ and $t_{M}=60 \mathrm{~min}$. All tests were duplicated.

\subsubsection{Scanning Electron Microscopy (SEM)}

Scanning electron microscopy (SEM) was used to image the arsenic-laden EGA sludge generated by ECAR treatment in synthetic Bangladesh groundwater. SEM images the sample surface by scanning it with a high-energy beam of electrons. The electrons interact with atoms on the surface of the sample providing information about the

\footnotetext{
${ }^{2}$ Pore size is determined using a bubble point test, in which liquid is held in pores by surface tension and the minimum pressure required to force the liquid from the capillary pore structure is directly related to the capillary diameter.
} 
topography and enabling a uniquely large depth of field in the resulting micrograph. Arsenic-laden EGA sludge was collected on a $0.1 \mu \mathrm{m}$ membrane after ECAR treatment at $j=1.1 \mathrm{~mA} / \mathrm{cm}^{2}$. The sample dried in air and aged 7-9 days by the time of imaging. A sludge coated membrane was coated in metallic gold and imaged using a Hitachi SE/N 4300 scanning electron microscope at Lawrence Berkeley National Lab (LBNL). An accelerating voltage of $15 \mathrm{kV}$ was used to obtain images at 500x, 50,000x, and 100,000x magnification. Energy dispersive spectroscopy (EDS) was performed on several areas of the sample using a NORAM System Six (NSS; Thermo Scientific). Work with the scanning electron microscope was made possible by the excellent training and direct assistance of Dr. Eduardo Saiz of LBNL.

\subsubsection{Chemical analysis of EGA sludge}

During the Matrix 2 run of batch tests (described fully in Section 3.2.7), arsenicladen EGA sludge was collected onto $0.1 \mu \mathrm{m}$ membranes and dried ${ }^{3}$. The dry powder was scraped into glass test tubes and stored in open air. In one case $\left(j=0.020 \mathrm{~mA} / \mathrm{cm}^{2}\right)$, powder was collected from the iron electrode surface (after drying). Powder was lightly tapped off of the electrode rather than scraped in order to avoid scraping metallic iron flakes from the electrode itself.

All powder samples were dissolved in a 1.5:1 solution of $38 \%-\mathrm{HCl}$ acid: deionized water. The powder was fully dissolved after approximately 4 hours, and a 1:50 dilution was sent for ICPMS analysis of aqueous iron and arsenic (ICPMS described in

\footnotetext{
${ }^{3}$ Drying occurred in a glove box purged with $\mathrm{N}_{2}$ gas in conjunction with preparation of XAS samples (described in Section 4.2.4. However the powder was later exposed to air.
} 
Section 3.2.1). Reproducibility of this procedure was tested by sending four samples prepared from the same powder, in each case dissolving a different amount from 25 $73 \mathrm{mg}$ per $10 \mathrm{~mL}$ solution. The resulting Fe/As mass ratios were within $4 \%$ of eachother.

In one case, the total mass of sludge produced was measured using a precision balance (up to $0.0001 \mathrm{~g})^{4}$. However, $\sim 1 \mathrm{mg}$ may have been lost due to flaking limiting the precision of the measurement.

\subsubsection{X-ray Absorption Spectroscopy (XAS)}

\subsubsection{Overview}

X-ray Absorption Spectroscopy (XAS) consists of recording the absorption of X-rays by target atoms as a function of the X-ray beam energy (eV) to create an XAS spectra. Spectral features near the absorption edge appear within the X-ray Absorption Near-Edge Structure (XANES) spectrum. Farther from the absorption edge, an oscillatory signal is produced by the backscattering of ejected photoelectrons by atoms immediately surrounding the target atom. The oscillatory signal is called the Extended X-ray Absorption Fine Structure (EXAFS) spectrum. Given the short mean free path of the ejected photoelectron (a few angstroms for a kinetic energy of a few hundred eV) structural information can be discerned form the first atomic shells surrounding the Xray absorber. This ability makes EXAFS analysis a unique tool for elucidating the local structure of poorly crystallized particles and surface complexes (Charlet \& Manceau, 1993). The EXAFS spectrum, $\chi$ is a function of the photoelectron wave vector, $k$, where

\footnotetext{
${ }^{4}$ The total mass of sludge was not weighed in every case because some sludge was removed to prepare XAS samples before drying - described in Section 4.2.4 below.
} 
$k$ is related to the kinetic energy of the photoelectron (E) by:

$$
k=\left[\frac{2 m}{\hbar^{2}}\left(E-E_{0}\right)\right]^{1 / 2}
$$

where $E_{0}$ is the threshold energy of the photoelectron at $k=0$, and $m$ is the mass of the electron. A typical form of the EXAFS function for a single set of $N_{R}$ atoms at a distance $\mathrm{R}$ from the absorbing atom is given by (O'Day et al., 1994):

$$
\chi(k)=\sum_{r} N_{R} S_{0}^{2} \frac{\left|f_{e f f}(\pi, k, R)\right|}{k R^{2}} \sin \left(2 k R+2 \delta^{c}+\Phi\right) e^{-2 \sigma^{2} k^{2}} e^{-2 R / \lambda}
$$

where $f_{\text {eff }}(\pi, k, R)$ is the effective curve-wave backscattering amplitude of the scatterer (Teo \& Joy, 1981), $\delta^{c}$ and $\Phi$ are phase shifts for the absorber and backscatterer respectively, $S_{0}^{2}$ is a many-body amplitude reduction factor, $\sigma^{2}$ is a Debye-Waller term (or a mean-square relative displacement) assuming a harmonic oscillator, and $\lambda$ is the mean free path of the electron. Theoretical functions for the phase $\left(\delta^{c}\right.$ and $\left.\Phi\right)$ and amplitude $\left(f_{\text {eff }}(\pi, k, R)\right)$ are generated from ab initio theory by the program FEFF (Rehr et al., 1991) using model compounds of similar structure. This allows extraction of the values $N_{R}, R$, and $\sigma^{2}$ from the EXAFS function.

For this work, EXAFS and XANES spectra were collected over four short runs at two synchrotron facilities. The sample preparation procedure was refined and improved with each run, as described below. 


\subsubsection{Iron XANES and XRF-maps}

\section{Sample preparation}

Fe XANES samples were prepared from the EGA sludge and the surface of the iron electrode after ECAR treatment ${ }^{5}$ of SBGW-1 synthetic groundwater ${ }^{6}$ at various operating conditions (listed in Table 4.1). The initial $\mathrm{pH}$ of the synthetic groundwater before ECAR treatment was $\mathrm{pH}=7.80$, slightly higher than the average $\mathrm{pH}$ of SBGW-1 used in Matrix 1 batch tests ${ }^{7}$. Sludge samples were collected after the ECAR mixing stage on polyvinylidene fluoride membrane filters (Millipore; $0.1 \mu \mathrm{m}$ pore size) and dried in air. Electrode surface layer rubbing samples were collected by pressing Kapton tape to the wet electrode surface immediately after ECAR dosing. All EGA sludge were allowed to dry in air (individual sample ages are listed in Table 4.1), though it should be noted that in several cases, samples were prepared at the beamline and placed in the beam wet and within 5 minutes of completing ECAR treatment (the listed age for these samples is 0 days).

Note that ECAR treatment at $j=5.0$ and $0.02 \mathrm{~mA} / \mathrm{cm}^{2}$ was performed in a much smaller electrochemical cell than the than the $850 \mathrm{~mL}$-separated cathode used for Matrix 1 experiments. The anode was formed from an iron wire (diameter $0.18 \mathrm{~cm}$ ) coated in epoxy resin and sanded at one end - exposing an iron disk of area $0.025 \mathrm{~cm}^{2}$. The cathode was a platinum wire coil approximately $2 \mathrm{~mm}$ away from the anode. The cell was a small glass test tube containing $220 \mu \mathrm{L}$ of solution, forcing the same $\mathrm{A} / \mathrm{V}$ ratio of

\footnotetext{
${ }^{5}$ The ECAR treatment procedure is detailed in Section 3.2.5. The ECAR operating parameters are summarized in Section 1.4.1.

${ }^{6}$ The composition of SBGW-1 is given in Table 3.1 .

${ }^{7}$ Matrix 1 batch tests are presented in Chapter 3 .
} 
the $850 \mathrm{~mL}$-separated cathode cell used in Matrix 1 experiments $\left(\mathrm{A} / \mathrm{V}=0.118 \mathrm{~cm}^{-1}\right)$. The solution was not continuously stirred during the treatment or the mixing stage, though the test tube was lightly shaken periodically during both processes. Before ECAR treatment, the anode was conditioned for 6 minutes at a constant current of $-1 \mathrm{nA}$ to electrochemically reduce any oxide layer that may have formed on the electrode while sitting in air. The small cell was designed to facilitate grazing incidence spectroscopy, described below.

\section{Data collection}

Fe k-edge XANES spectra and spatially-resolved X-ray fluorescence (XRF) maps were collected under ambient conditions on beamline 10.3.2 at the Advanced Light Source (ALS; Berkeley, CA) with a 7-element germanium detector in fluorescence mode (Marcus et al., 2004). Ring conditions were 200-400mA and $1.9 \mathrm{GeV}$. Monochromator energy calibration was set with an $\mathrm{Fe}(0)$ foil at $7110.75 \mathrm{eV}$. XRF elemental maps were collected at an incident energy of $12067 \mathrm{eV}$ (i.e. $200 \mathrm{eV}$ above the As K-edge) with a pixel size of $5 \times 5 \mu \mathrm{m}$ and a dwell time of $100 \mathrm{~ms}$ per pixel. Maps were deadtime corrected and registered using custom beamline software developed by M. Marcus (ALS). The maps were used to locate regions of interest for collection of Fe XANES spectra. Experimental spectra were deadtime corrected and energy calibrated using custom beamline software developed by M. Marcus (ALS). 


\section{A note on grazing incidence spectroscopy}

The original purpose for choosing the 10.3.2 micro-beam at ALS was to perform grazing incidence spectroscopy on the electrode surface immediately after ECAR dosing. Grazing incidence spectroscopy takes advantage of the fact that the penetration depth of the X-ray beam is controlled by the glancing angle - if the angle can be controlled enough to cause a total reflection, the penetration depth can be suppressed to the order of a few nm (Sato et al., 2005). The beam footprint is lengthened by the grazing angle, making a small beam such as the micro-beam 10.3.2 at ALS, ideal to obtain maximum flux. Performing spectroscopy on the electrode itself immediately after treatment was intended to minimize disruption and aging of the surface layer, leading to a more accurate picture of the surface layer during the ECAR dosing stage.

The attempt to take spectra at grazing incidence ultimately failed as the signal from the surface layer was too weak to compete with the Fe(0) signal of the electrode itself. A steeper grazing angle (leading to a smaller penetration depth) was not able to compensate because the electrode surface was neither uniform enough nor smooth enough for the entire beam area to penetrate to the same depth at a steep angle. One suggestion for looking at the surface directly is to sputter a uniform and thin layer of iron onto an inert substrate and perform in-situ ECAR treatment while measuring the

EXAFS spectra. Because X-rays penetrate an appreciable distance through water, it is possible to perform surface studies at the electrolyte-solid interface (Tang \& Furtak, 1991). Building and testing such a cell was unfortunately beyond the scope of this work. This section is included for the benefit of future investigators. 
Table 4.1: ECAR operating conditions used to generate XAS samples. ECAR operating conditions (summarized in Section 1.4.1) include current density, charge density, current processing time $\left(t_{C P}\right)$, and mixing time $\left(t_{M}\right)$. XAS sample preparation procedures are described in Sections 4.2.4.2 - 4.2.4.5.

\begin{tabular}{|c|c|c|c|c|c|c|c|c|}
\hline $\begin{array}{l}\text { Spectra } \\
\text { Type }\end{array}$ & $\begin{array}{c}\text { Current } \\
\text { Density } \\
\left(\mathrm{mA} / \mathrm{cm}^{2}\right)\end{array}$ & $\begin{array}{l}\text { Sample } \\
\text { Type }^{\mathrm{b}}\end{array}$ & $\begin{array}{c}\text { Beam } \\
\text { Location }^{\mathrm{c}}\end{array}$ & $\begin{array}{c}\text { Charge } \\
\text { Density } \\
(\mathrm{C} / \mathrm{L})\end{array}$ & $\begin{array}{c}t_{C P} \\
(\min )\end{array}$ & $\begin{array}{c}t_{M} \\
(\min )\end{array}$ & $\begin{array}{l}\text { SBGW } \\
\text { Recipe }^{d}\end{array}$ & $\begin{array}{l}\text { Sample } \\
\text { Age }^{\mathrm{e}} \\
\text { (days) }\end{array}$ \\
\hline Fe XANES & 1.1 & EGA sludge & ALS & 100 & 12.8 & 60 & 1 & 1 \\
\hline Fe XANES & 5 & Elec SL & ALS & 100 & 2.83 & 40 & 1 & 0 \\
\hline Fe XANES & 0.02 & EGA sludge & ALS & 100 & 708 & 40 & 1 & 0 \\
\hline Fe XANES & 0.02 & Elec SL & ALS & 100 & 708 & 40 & 1 & 0 \\
\hline Fe XANES & 0.07 & EGA sludge & ALS & 100 & 203 & 60 & 1 & 2 \\
\hline Null As EXAFS & 1.1 & Elec SL & SSRL & 150 & 66.58 & 60 & 2 & 5 \\
\hline As EXAFS & 5 & EGA sludge & SSRL & 175 & 16.33 & 60 & 2 & 3 \\
\hline Fe EXAFS & 1.1 & EGA sludge & ALS & 175 & 25.58 & 60 & 2 & 2 \\
\hline Fe EXAFS & 0.02 & EGA sludge & ALS & 75 & 452.92 & 60 & 2 & 4 \\
\hline Fe EXAFS & 100 & EGA sludge & ALS & 175 & 3.17 & 60 & 2 & 4 \\
\hline Fe EXAFS & 5 & EGA sludge & ALS & 175 & 5.63 & 60 & 2 & 2 \\
\hline
\end{tabular}

a Null As EXAFS refers to attempts to generate As k-edge EXAFS spectra that failed to produce any As signal.

b Sample type can be either EGA Sludge, referring to samples collected from the bulk electrolyte, or Elec $S L$, referring to samples collected from the electrode surface layer after ECAR treatment.

b Beam location is either ALS (Advanced Light Source - Berkeley, CA) or SSRL (Stanford Synchrotron Radiation Laboratory- San Jose, CA).

d Synthetic Bangladesh Groundwater (SBGW) recipes 1 and 2 are described in Table 3.1.

e Sample age refers to the time between sample preparation and spectra collection. 


\subsubsection{Null arsenic EXAFS attempts}

Null arsenic EXAFS attempts refer to several attempts to generate As k-edge EXAFS spectra from electrode surface layer scrapings that failed to produce any As signal. These results are significant in constraining the location of arsenic removal in ECAR (i.e. on the electrode surface or within the bulk electrolyte), and as such, the sample preparation procedure and attempted data collection are described here.

\section{Sample preparation}

Electrode surface layer samples were collected after ECAR treatment of SBGW2 synthetic groundwater ${ }^{8}$ during Matrix 2 batch experiments ${ }^{9}$ at various operating conditions (listed in Table 4.1). After the dosing stage of ECAR treatment, the iron anode was immediately transferred to an anaerobic chamber for drying. Within the anaerobic chamber, the dry electrode surface layer was carefully scraped with a clean Xacto knife blade down to the metal electrode ${ }^{10}$. In the case of dosing at $j=0.02 \mathrm{~mA} / \mathrm{cm}^{2}$, the electrode surface was caked with a thick $(\sim 0.5 \mathrm{~mm})$ layer of orange powder that easily flaked off after drying without the use of a knife blade. Electrode surface layer scrapings were packed into glass capillaries with a $2 \mathrm{~mm}$ outer diameter. The estimated thickness of the glass itself is $0.25 \mathrm{~mm}$ (by eye $\mathrm{e}^{11}$ ). Capillaries were capped with epoxy resin and stored at ambient temperature in the anaerobic chamber until transfer to the beam

\footnotetext{
${ }^{8}$ The composition of SBGW-2 is given in Table 3.1.

${ }^{9}$ Matrix 2 batch tests are presented in Chapter 3.

${ }^{10}$ Note that in most cases, this procedure is not appropriate for Fe k-edge spectra because flakes of $\mathrm{Fe}(0)$ from the electrode or the stainless steel knife blade can easily contaminate the sample.

${ }^{11}$ The exact thickness is unknown because the capillaries were hand-pulled rather than purchased from a manufacturer.
} 
between 5-9 days after collection (individual sample ages are listed in Table 4.1).

It should be noted that after a reconnaissance trip to the beam, capillaries were found to be to too long for the sample chamber and the samples were repacked into new shorter glass capillaries (same outer diameter) 2 days before data collection. This relevant because during the repacking, it was noted that the electrode surface scrapings were far more magnetic than they had been during the first packing. This is a sign that the iron (hydr)oxides were aging into more crystalline magnetite or maghemite (both magnetic) during anaerobic storage.

\section{Data collection attempts}

Arsenic k-edge EXAFS collection was attempted with an unfocused beam (beamsize $2 \times 20 \mathrm{~mm}$ ) under ambient conditions at beamline 11-2 at the Stanford Synchrotron Radiation Laboratory (SSRL; San Jose, CA). Ring conditions were 50 - 100 mA at 3GeV. The beam was detuned $30 \%$ to avoid harmonics. Energy calibration was performed with an arsenic metal foil at $11867 \mathrm{eV}$. Beam energy was selected using a $\mathrm{Si}(220)$ monochromator and fluorescence signals were generated using a 30-element Germanium detector. In all cases, transmission and fluorescence signals were measured simultaneously. Stoller slits and Ge filters were used in front of the fluorescence detector to minimize the role of Compton scattered primary X-rays. In each attempt to find an arsenic signal, the beam

was physically centered on the sample using the easily detectable Fe peak and the slits were opened as wide as possible $(2 \times 20 \mathrm{~mm})$ at a beam energy $200 \mathrm{eV}$ above the As k-edge. Various combinations of filters (i.e. Al and Ge) were used to help resolve any 
possible As signal. Successful As k-edge EXAFS spectra were measured for the electrode scrapings from $j=0.02 \mathrm{~mA} / \mathrm{cm}^{2}$, proving that the setup was in proper working order for As detection ${ }^{12}$.

\subsubsection{Arsenic k-edge EXAFS}

\section{Sample preparation}

EGAsludge samples were collected after ECAR treatment of SBGW-2 synthetic groundwater $^{13}$ during Matrix 2 batch experiments at various operating conditions (listed in Table 4.1). After the ECAR mixing stage, sludge from $\sim 1$ liter of dosed and mixed solution was collected on a filter membrane $(0.1 \mu \mathrm{m}$ pore size; membrane filtration took $\approx$ 1 hour). Once filtration was complete, the wet sludge was transferred into the anaerobic chamber while submerged in filtrate solution (keeping the sludge wet during the 10 minute transfer into the chamber). Once in the chamber, wet sludge was scraped from the membrane onto Kapton film and formed into a pill shape in order to concentrate the arsenic as much as possible. A smaller piece of Kapton film was placed on top and the edges sealed with epoxy resin to maintain a wet paste consistency. Two identical paste preparations were prepared for each sludge sample. After 5 minutes (to allow the epoxy to dry), the sealed paste samples were sealed inside $20 \mathrm{~mL}$ glass jars (to slow oxygen contamination through the Kapton film) and transferred from the anaerobic chamber into a $1^{\circ} \mathrm{C}$ storage unit. The $20 \mathrm{~mL}$ glass jars were later placed inside $150 \mathrm{~mL}$

\footnotetext{
${ }^{12}$ As EXAFS spectra for $j=0.02 \mathrm{~mA} / \mathrm{cm}^{2}$ electrode scrapings were later remeasured using a different sample preparation procedure, described in Section 4.2.4.4.

${ }^{13}$ The composition of SBGW-2 is given in Table 3.1.
} 
plastic bottles (within the anaerobic chamber) to act as an additional barrier to oxygen contamination during storage. Samples were stored for 3-7 days (individual sample ages are listed in Table 4.1).

\section{Data collection}

Arsenic K-edge EXAFS spectra were taken on beamline 11-2 at SSRL under the same beam conditions described in Section 4.2.4.3. However, in this case, samples were removed from protective jars and placed immediately into a small anaerobic cell where they remained during the EXAFS scan. All wet paste samples appeared wet (by eye and touch) before and after EXAFS spectra collection. Spectra were collected using a beam size of $2 \times 20 \mathrm{~mm}$ to a $\mathrm{k}$-value of $14 \AA^{-1}$. Initially, beam harmonics were reduced using grazing incidence cut-off reflection mirrors to eliminate higher order radiation. However,

it was discovered during the run that spectral data above $k=11 \AA^{-1}$ was extremely noisy and non-reproducible between scans. The mirrors were removed and the beam was detuned 30\% to reduce harmonics, as was the case during the first SSRL run (described in Section 4.2.4.3). This reduced the noise considerably and led to reproducible data. All data presented here used detuning, however this note was included to inform future EXAFS data collectors. 


\subsubsection{Iron k-edge EXAFS}

\section{Sample preparation}

Sample preparation for Fe k-edge EXAFS was the same as for As k-edge EXAFS (Section 4.2.4.4), with the following exceptions: (1) Saran wrap was sandwiched inside the Kapton film surrounding each sample to further protect against oxygen contamination, (2) sealed Kapton/Saran film samples were placed inside $20 \mathrm{~mL}$ glass jars and $150 \mathrm{~mL}$ plastic jars immediately after being formed inside the anaerobic chamber neither jar was opened until just before EXAFS data collection, and (3) instead of scraping the wet sludge off of the membrane, a small piece of wet sludge-covered membrane was cut into strips and sealed within the Kapton/Saran wrap film. The last modification was done to provide a more uniform thin sludge layer ${ }^{14}$. All samples were stored at $1^{\circ} \mathrm{C}$ until EXAFS data collection.

\section{Data collection}

Iron k-edge EXFAS spectra were measured at beamline 10.3.2 at ALS. Ring conditions were 200 - $400 \mathrm{~mA}$ at $1.9 \mathrm{GeV}$. The beam was detuned $20 \%$ to avoid harmonics. Energy was calibrated to $\mathrm{Fe}(0)$ foil at $7110.75 \mathrm{eV}$. Spectra were collected using a beam size of $100 \times 10 \mu \mathrm{m}$ to $100 \times 20 \mu \mathrm{m}$. Individual scans collected at the same location were examined for changes in line shape and peak position and no beam radiation damage was observed. Spectra were collected to a k-value of $14 \AA^{-1}$. However, it was discovered during EXAFS collection that data past $\mathrm{k} \sim 11 \AA^{-1}$ had very low signal-to-noise and was

\footnotetext{
${ }^{14}$ Note also that previous samples were concentrated to get a better signal from trace arsenic. Iron was much more abundant in the samples, and therefore a thin layer provided plenty of iron signal.
} 
not reproducible between scans. This may have been due to the wet samples settling in gravity during the scan, effectively changing the beam penetration depth across the scan. The EXAFS from each scan were compared by eye and the average data was cut off to an appropriate k-value such that data was consistent across scans. Acceptable k-values were between $\mathrm{k}=10.5$ and $11.2 \AA^{-1}$ Experimental spectra were deadtime corrected and energy calibrated using custom beamline software developed by M. Marcus (ALS).

It should be noted that the micro-beam available at 10.3 .2 was not ideal for these samples. The EGA sludge is quite uniform, negating the spacial resolution power of a micro-beam. In addition, the increased flux produced by a larger beam would allow spectra to be taken much more quickly and possibly without some of the problems at high $\mathrm{k}$ discussed above. Beamline 10.3.2 was used because time on a bulk beam was not otherwise available ${ }^{15}$.

\subsubsection{Reference spectra}

Reference Fe XANES spectra for synthetic iron (hydr)oxides, including 2-line ferrihydrite, 6-line ferrihydrite, goethite, lepidocrocite, akaganeite, magnetite, and maghemite, along with Fe metal foil, were collected at beamline 10.3.2 at ALS by Dr. Matthew Marcus (ALS) and provided via personal communication. Spectra were received averaged, deadtime corrected, and energy calibrated.

Reference Fe EXAFS and Fe XANES spectra for synthetic samples of scorodite $\left(\mathrm{FeAsO}_{4} \cdot 2 \mathrm{H}_{2} \mathrm{O}\right)$, carbonate green rust $\left(\left(\mathrm{Fe}[\mathrm{II}]_{4} \mathrm{Fe}[\mathrm{III}]_{2}\right)(\mathrm{OH})_{12} \mathrm{CO}_{3}\right)$, and sulfate green

\footnotetext{
${ }^{15}$ Dr. Matthew A. Marcus and Ms. Sirine Fakra deserve thanks for making the micro-beam work as well as it could under non-optimal conditions.
} 
rust $\left(\left(\mathrm{Fe}[\mathrm{II}]_{4} \mathrm{Fe}[\mathrm{III}]_{2}\right)(\mathrm{OH})_{12} \mathrm{SO}_{4}\right)$ were provided by Dr. Peggy O'Day (Arizona State University). Sample preparation and data collection are described in O'Day et al. (1994). Spectra were received averaged, deadtime corrected, and energy calibrated.

Reference As EXAFS spectra for arsenate (As[V]) adsorbed onto 2-line ferrihydrite, arsenate adsorbed onto goethite, and scorodite were provided by Dr. Andrea Foster (US Geological Survey). Sample preparation and data collection are described in Foster (1999). Spectra were received averaged, deadtime corrected, and energy calibrated.

While all of the above authors were kind enough to offer me their spectra for my thesis work, they have neither read nor approved of the analysis based on comparisons to the above spectra, and thus it should not be taken to reflect their professional opinion.

\subsubsection{XAS data analysis}

Spectra averaging was performed using in SIXPack software (Webb (2003); SIXPack v2.3.1) for arsenic EXFAS spectra and using "EXAFS editor.exe" developed by M. Marcus (ALS) for iron XANES and iron EXAFS spectra. In the case of iron EXAFS spectra, each scan in the average was viewed side by side to determine an appropriate data cut off point based on reproducibility of the signal between scans (see discussion in Section 4.2.4.5). All averaged, unsmoothed EXAFS spectra were background-subtracted by a linear fit through the pre-edge region and a cubic spline through the spectrum above the absorption edge using SIXPack. A threshold energy (i.e. the energy at which $\mathrm{k}$, the photoelectron wave vector, equals zero) of $7132 \mathrm{eV}$ was used for iron and $11873 \mathrm{eV}$ was

used for arsenic. XANES spectra were normalized using the height of the edge step just 
above the absorption maximum using "EXAFS editor.exe." All reference spectra from outside sources were re-processed using the same methods as sample spectra used in comparisons.

\subsection{Results and Discussion}

\subsubsection{Limit on the average EGA cluster size}

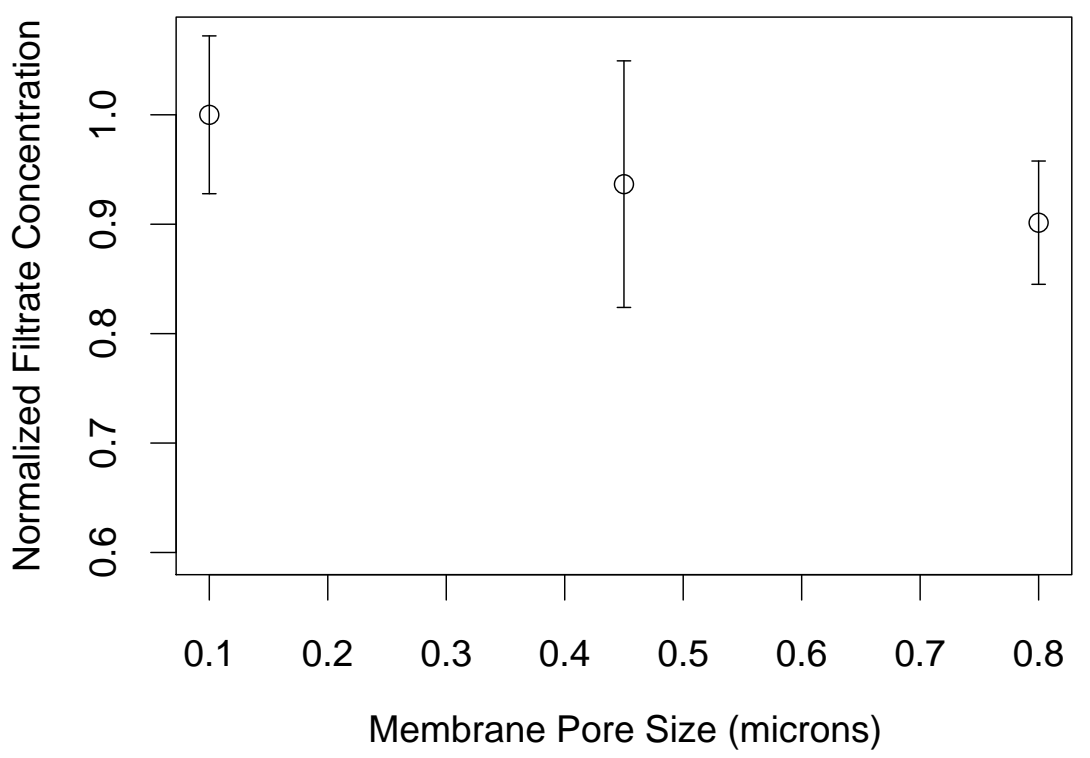

Figure 4.1: Arsenic concentration measured in filtrate (normalized to the highest average value) after ECAR-treated sample water passed through membranes of different pore size. Errors represent the variability between duplicate tests.

A rough lower limit on the average size of EGA clusters can be determined through filtration tests by progressively increasing the pore size of the filter membrane. Filtrate arsenic concentrations after filtration with $0.1-0.8 \mu \mathrm{m}$ pore size membranes were measured in SBGW-1 after ECAR treatment at $j=0.70 \mathrm{~mA} / \mathrm{cm}^{2}, q=14.8 \mathrm{C} / \mathrm{L}$, 
$t_{C P}=3 \min , t_{M}=60-135 \min$ (Figure 4.1). A small decrease in the effluent arsenic was observed, though this decrease was smaller than the variability between duplicate tests (indicated by error bars), resulting in no significant difference across pore sizes. A decrease in effluent arsenic is also unlikely, since a larger pore size should never lead to the passage of fewer particles. Some room for variability was introduced by allowing the mixing time to vary between filtrations, as clusters are likely to get larger with increasing mixing time. However, filtration occurred in order of decreasing pore size, and thus any bias would tend to lead inflate effluent concentrations at higher pore size. Thus this data, while not ideal, implies that the cluster size of EGA is greater than $0.8 \mu \mathrm{m}$. This result is significant largely because similar experiments reported in the literature often use $0.45 \mu \mathrm{m}$ pore size filtration, which can now be robustly compared to batch test results using $0.1 \mu \mathrm{m}$ filtration. It also increases the measured minimum cluster size by a factor 8 , which is relevant for selecting low cost alternatives to $0.1 \mu \mathrm{m}$ membrane filtration for a prototype device.

\subsubsection{Scanning Electron Microscopy (SEM)}

ECAR generates an iron (hydr)oxide sludge used to capture arsenic. The sludge, collected on a $0.1 \mu \mathrm{m}$ polyvinylidene fluoride membrane, was imaged at $500 \mathrm{x}$ magnification using SEM (shown in Figure 4.2A). At 500x magnification, the sludge appears to be a relatively uniform cake (showing cracks due to drying) dotted with small square crystals. Marked areas were imaged at higher magnification, including one area with a square crystal (shown at 8,000x magnification in Figure 4.2B) and one area con- 
taining caked powder (shown at 50,000x and 100,000x magnification in Figure 4.2C and D respectively). Energy dispersive spectra (EDS) were taken of the magnified images ${ }^{16}$, shown in Figure 4.3B and $\mathrm{C}$ corresponding to Figure 4.2B and $\mathrm{C}$ respectively, along with one spectra of the bare polyvinylidene fluoride membrane (Figure 4.3A; bare membrane image not shown). The bare membrane spectra shows the characteristic Au lines corresponding to the gold coating used in the SEM process, and no appreciable calcium, arsenic, or iron. The spectra of the square crystal (Figure 4.3B) shows peaks only due to the gold coating and calcium implying that the crystals are made of calcium, presumably $\mathrm{CaCO}_{3(\mathrm{~s})}$. Notably, no iron is associated with these crystals, indicating that the crystals are not a product of ECAR treatment. As expected, the caked powder contains appreciable amounts of iron along with some arsenic (shown in Figure 4.3C), identifying the magnified caked powder in Figure $4.2 \mathrm{C}$ and $\mathrm{D}$ as the iron (hydr)oxides product of ECAR treatment (i.e. as EGA). The presence of arsenic also verifies that some arsenic is removed in the sludge as opposed to all being adsorbed to the surface layer of the electrode.

Magnified images of EGA (Figures 4.2C and 4.2D) show that the iron (hydr)oxides particles are rounded, consisting of tiny spheres around $25 \mathrm{~nm}$ in diameter forming larger popcorm-like clumps with a primary aggregate size of 50 - $100 \mathrm{~nm}$. Only one particle shape can be identified in areas associated with iron on the micrographs, suggesting that the iron (hydr)oxide composition of EGA is homogeneous. Filtration tests using membranes with pore sizes between $100 \mathrm{~nm}$ and $800 \mathrm{~nm}$ (Section 4.2.1) showed no increase

\footnotetext{
${ }^{16}$ The spectrum of the crystal shown in Figure 4.2B was taken at a higher magnification than $8,000 \mathrm{x}$ and was centered on a bare section of crystal.
} 


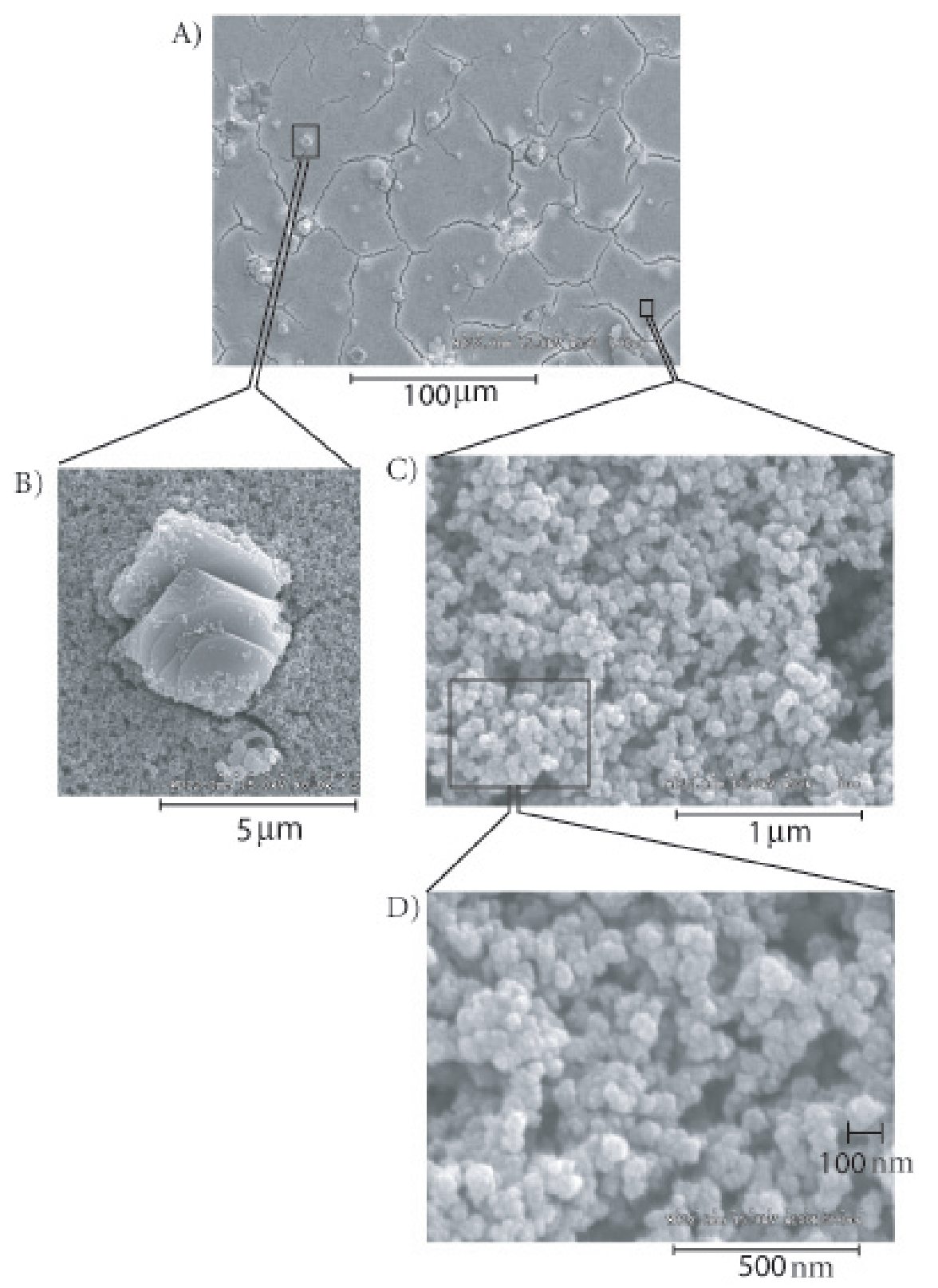

Figure 4.2: Scanning electron micrographs of ECAR generated sludge. Image $A$ is of air-dried sludge collected on a membrane and magnified 500x. The two marked areas in image $A$ are imaged at 8,000 x (image $B$ ) highlighting a representative square crystal and at 50,000x and 100,000x (image $C$ and $D$ respectively), highlighting a representative area of dry caked powder. 

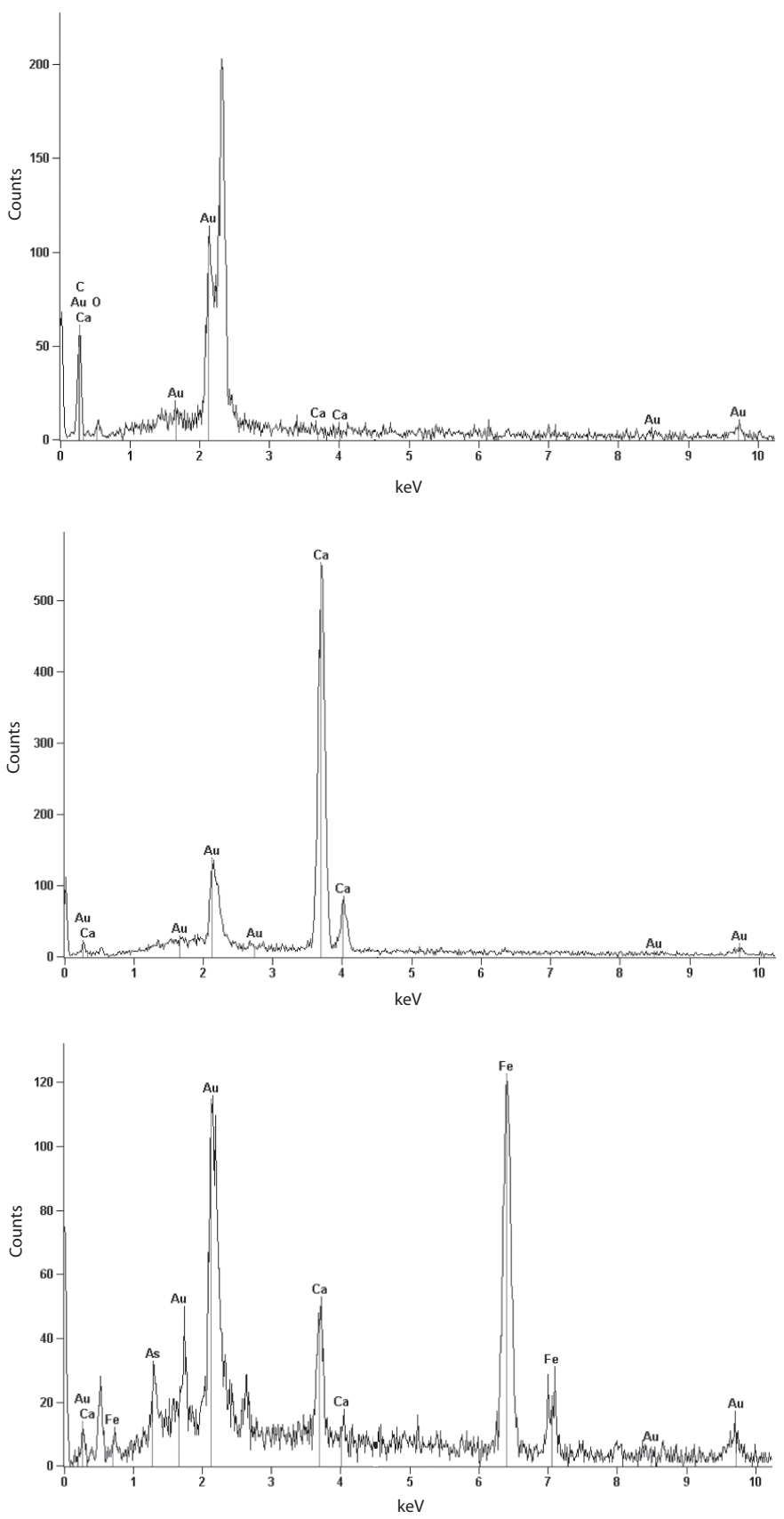

Figure 4.3: Energy dispersive spectra taken during SEM imaging of (A) the bare polyvinylidene fluoride membrane (background), (B) a further magnified image of Figure $4.2 \mathrm{~B}$ (square crystal) centered on a bare section of crystal, and $(\mathrm{C})$ the area shown in Figure 4.2D (caked powder). Au lines appear in all spectra due to the gold coating sputtered onto the sample to facilitate SEM imaging. 
in the arsenic concentration of the filtrate, suggesting that the cluster size of EGA is greater than $800 \mathrm{~nm}$.

Iron (hydr)oxides come in many different crystal shapes, and shape alone is not a unique identifier. However, it is worthwhile to note that out of the iron (hydr)oxides that appear at ambient temperature and pressure (e.g. goethite, lepidocrocite, akaganeite, ferrihydrite, hematite, magnetite, and maghemite), only ferrihydrite and occasionally magnetite and hematite appear as spheres. Goethite, known to form from amorphous- $\mathrm{Fe}(\mathrm{OH})_{3}$ during aging, commonly appears in an elongated acicular shape (Cornell \& Schwertmann, 2000). Hematite, also known to form from amorphous-Fe(OH) ${ }_{3}$, can appear as spheres, but most commonly appears as rounded or rhombohedral plate structures (i.e. flat as opposed to spherical). Ferrihydrite is characterized by small single spherical particles, ca. 4-6 nm in size. While not conclusive, it is interesting to note that after 7-9 days or aging in air, the ECAR generated sludge is consistent in appearance with ferrihydrite and does not appear to be either crystalline goethite or hematite.

\subsubsection{XRF Fe-As mapping of EGA sludge}

XRF mapping was done of the dry EGA sludge generated at $j=0.02,1.1$, and $5.0 \mathrm{~mA} / \mathrm{cm}^{2}$ at a pixel size of $2 \mu \mathrm{m}$. A representative map is shown in Figure 4.4 (maps at all current densities were similar). In this picture, each pixel is colored red in proportion to the As signal and green in proportion to the Fe signal (pixels with both arsenic and iron appear yellow). This map shows that both arsenic and iron are uniformly spread throughout the EGA sludge. The homogeneity of arsenic and iron 
seen here is similar to the homogeneity in particle shape and size seen in SEM images (Figure 4.2). Taken together, these data suggest that the EGA generated for a given current density is composed of iron (hydr)oxides of a similar structure and affinity for arsenic.



Figure 4.4: XRF bicolor map of EGA powder. In this picture, each pixel is colored red in proportion to the As signal and green in proportion to the Fe signal (pixels with both arsenic and iron appear yellow). This map was measured for EGA generated at $j=$ $1.1 \mathrm{~mA} / \mathrm{cm}^{2}$. Maps of EGA generated at $j=0.02$ and $5.0 \mathrm{~mA} / \mathrm{cm}^{2}$ were similar.

\subsubsection{Chemical analysis of waste EGA sludge}

The waste product of ECAR treatment is arsenic-laden EGA sludge (filtered out of the bulk solution). The leftover EGA sludge after ECAR batch treatment at various operating conditions was analyzed for Fe and As using ICPMS, listed in Table 4.2. The ratio of $\mathrm{As} / \mathrm{Fe}$ in the waste sludge depends on the coulombic dose (charge density), and thus should only be compared between batch tests with same total charge density. Also note that in one case $\left(j=0.02 \mathrm{~mA} / \mathrm{cm}^{2}\right)$, the electrode surface layer was collected and tested in addition to the EGA sludge from the bulk solution. 
Table 4.2: Chemical analysis of waste EGA sludge or electrode scrapings from ECAR treatment at various operating conditions. Sample preparation is described in Section 4.2.3.

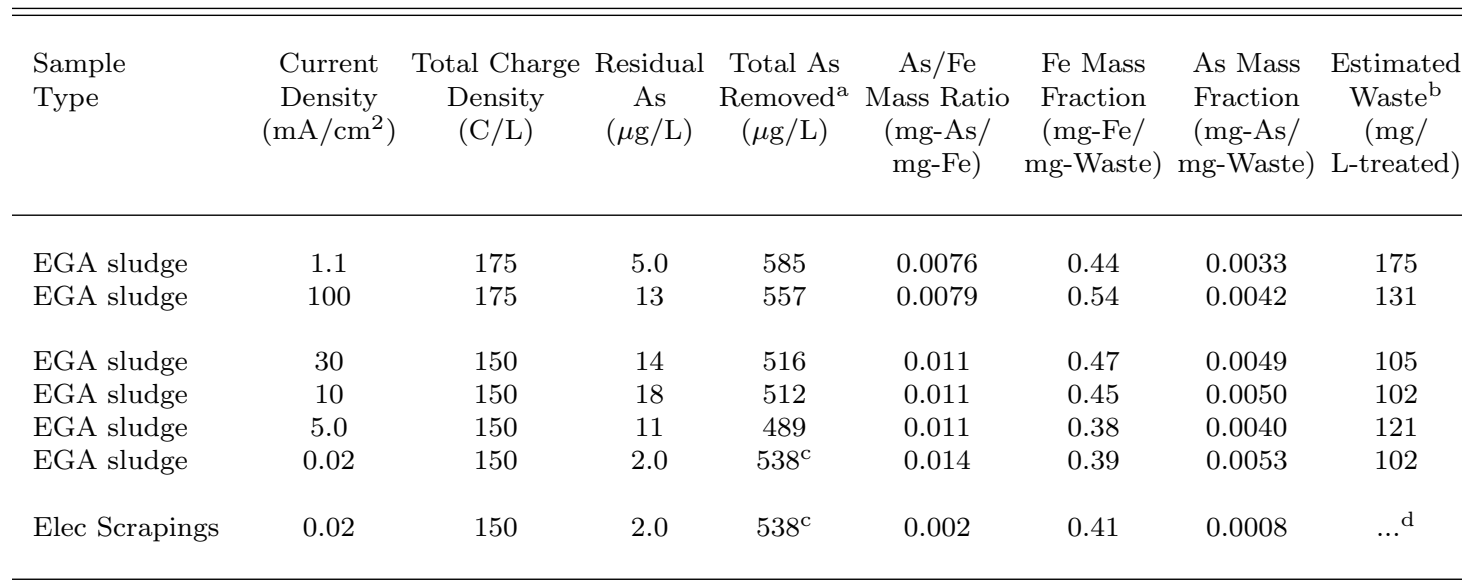

a This is the total arsenic removed during ECAR treatment - i.e. the initial aqueous arsenic concentration minus the final aqueous arsenic concentration.

b Estimated Waste uses the measured arsenic mass fraction (in mg-As/mg-Waste) and assumes all arsenic removed for the batch was removed in either the sludge or electrode scrapings waste (i.e. it is the arsenic removed divided by arsenic mass fraction).

c Note that this is the total arsenic removed from solution during ECAR treatment, including arsenic removed on the EGA sludge and on the electrode surface.

$\mathrm{d}$ The assumptions used to estimate the waste are not valid for electrode scrapings - see note b. 
As expected, the As/Fe ratio decreases with increasing charge density due to the additional iron dissolution. In addition, the As/Fe ratio will increase with the concentration of arsenic removed from the solution, creating some variation in the measured results due to the large variation in arsenic removed. The waste characteristics for ECAR treatment at $j=1.1 \mathrm{~mA} / \mathrm{cm}^{2}, q=175 \mathrm{C} / \mathrm{L}$ provide the best upper limit for the waste characteristics expected in Bangladesh because (1) the initial arsenic was higher than that measured in most tube wells $\left(>98 \%\right.$ of contaminated $^{17}$ tube wells contain less than $600 \mu \mathrm{g} / \mathrm{L}$ arsenic) and (2) the residual arsenic concentration is less than the WHO limit of $10 \mu \mathrm{g} / \mathrm{L}$, which is the treatment goal for ECAR. Solid waste for this batch test is characterized by an arsenic concentration of $0.0033 \mathrm{mg}-\mathrm{As} / \mathrm{mg}$-Waste, or $3300 \mathrm{ppm}$ $(1 \mathrm{ppm}=1 \mathrm{mg}-\mathrm{As} / \mathrm{kg}$-Waste $)$, and an As/Fe mass ratio of 0.0076

The iron mass fraction is $38 \%-54 \%$ across all sludge samples (excluding the electrode scrapings sample), consistent with an iron (hydr)oxide (for comparison, the iron mass fraction for pure $\mathrm{Fe}(\mathrm{OH})_{3}$ is $52 \%$ ).

The measured As/Fe mass ratio in electrode scrapings for EGA generated at $j=0.02 \mathrm{~mA} / \mathrm{cm}^{2}$ is 7 times less than the As/Fe ratio found in the EGA sludge. This implies that the iron (hydr)oxide surface layer formed on the electrode has a much lower affinity for arsenic than the EGA sludge in solution. The mass of the surface layer is also much smaller than the mass of EGA put into solution, implying that the surface layer affinity for arsenic would have to be very much greater than the EGA affinity for most of the arsenic removal to occur on the electrode surface rather than in the bulk.

\footnotetext{
17" Contaminated tube wells" includes all tube wells with arsenic concentrations above the WHO limit of $10 \mu \mathrm{g} / \mathrm{L}$ according to the BGS (2001) - see Section 3.2.3 for more info on tube well statistics.
} 
The majority of arsenic removal in ECAR is thus likely to occur in the bulk rather than on the electrode surface.

A very rough estimate for the amount of waste expected per liter of contaminated water treated by ECAR (assuming a charge density of 150-175 C/L) is calculated in Table 4.2. This estimate assumes that the arsenic mass fraction is constant throughout the waste (a fair assumption given the homogeneity of EGA seen in SEM images and XRF mapping) and assumes that all of the arsenic removed in the batch test occurred in the bulk solution (i.e. on the EGA sludge). The estimated waste is $102-175 \mathrm{mg}$ per liter treated. In one case, the waste EGA sludge produced after ECAR treatment of 3 liters at a charge density of $150 \mathrm{C} / \mathrm{L}$ was directly weighed, resulting in $100 \pm 1 \mathrm{mg}$ waste, or $33 \mathrm{mg}$ waste per liter treated. The factor of 3 discrepancy between estimated waste and measured waste indicates that the amount of arsenic removed from solution is not a good predictor of waste and a better method should be explored.

\subsubsection{Iron oxidation state in EGA}

The peak position in XANES spectra is characteristic of the oxidation number of the absorbing atom (Foster, 2003). In some case, features present in XANES spectra are characteristic of a particular mineral, however, Fe[III] oxides and oxyhydroxides with Fe in octahedral coordination are known to have few distinguishing features (O'Day et al., 1994). The peak position can be used to distinguish between Fe[III](hydr)oxides and Fe(II, III)(hydr)oxides, but not to identify individual minerals within these two classes (O’Day et al., 1994). Normalized Fe XANES spectra for EGA sludge after ECAR treat- 
ment at several different current densities $\left(j=0.02,0.07,1.1\right.$, and $\left.5.0 \mathrm{~mA} / \mathrm{cm}^{2}\right)$ are shown in Figure 4.5 along with Fe XANES spectra for various iron (hydr)oxide reference compounds, including Fe[III](hydr)oxides: 2-line ferrihydrite (2L FH), 6-line ferrihydrite (6L FH), goethite, lepidocrocite, and akaganeite and Fe(II, III)(hydr)oxides: green rust (carbonate) and green rust (sulfate) (average oxidation number 2.67). The edge features of the EGA samples are similar to the Fe[III](hydr)oxide reference compounds, though the features are too similar to distinguish individual Fe[III](hydr)oxide minerals (as expected). However, a line through the peak position of the EGA samples clearly crosses the peak position of the Fe[III](hydr)oxides and not the peak position of the Fe(II, III)(hydr)oxides (green rusts). This suggests that the oxidation of iron in EGA generated at current densities $j=0.02,0.07,1.1$, and $5.0 \mathrm{~mA} / \mathrm{cm}^{2}$ is predominantly $3+$ and not a mixture of $2+$ and $3+$.

An oxidation state of $3+$ for EGA at $0.02 \mathrm{~mA} / \mathrm{cm}^{2}$ rules out the possibility of predominantly Fe[II]-based iron (hydr)oxides at low current density. This was suggested by the slow scan polarization curve based on thermodynamic equilibrium potentials in Section 3.3.10.

\subsubsection{EGA iron structure - comparison to known iron (hydr)oxides}

In Figure 4.6, the $\mathrm{k}^{3}$-weighted Fe EXAFS spectrum for EGA generated at $j=$ $1.1 \mathrm{~mA} / \mathrm{cm}^{2}$ is compared to reference spectra for iron (hydr)oxides 2-line ferrihydrite (2L FH), goethite, and scorodite. These reference spectra were chosen as the most similar to the sample spectrum out of a set including green rusts, hematite, magnetite, siderite, 


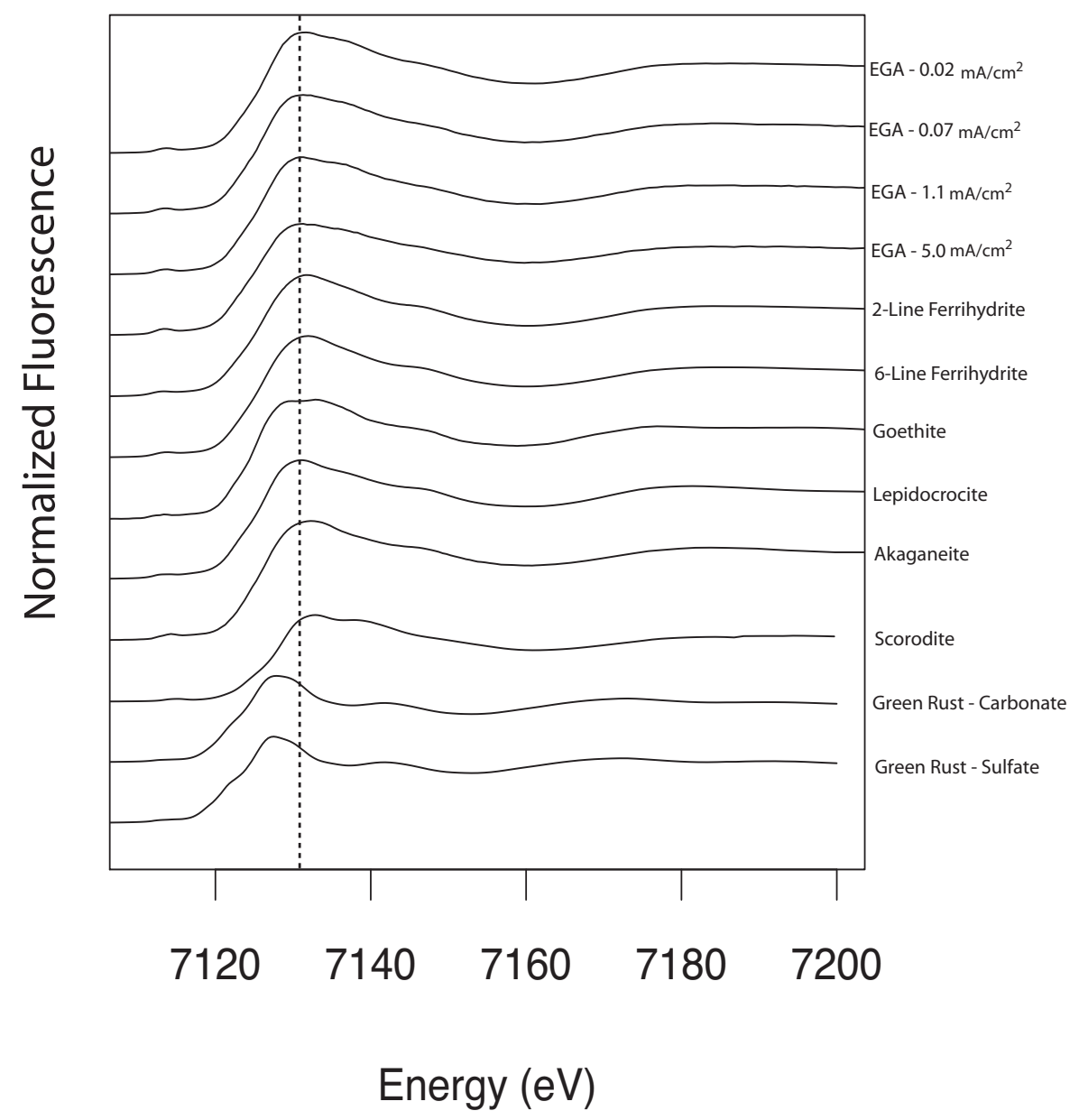

Figure 4.5: Normalized Fe XANES spectra for EGA sludge after ECAR treatment at several different current densities $\left(j=0.02,0.07,1.1\right.$, and $\left.5.0 \mathrm{~mA} / \mathrm{cm}^{2}\right)$ are shown along with Fe XANES spectra for various iron (hydr)oxides reference compounds, including Fe[III](hydr)oxides 2-line ferrihydrite, 6-line ferrihydrite, goethite, lepidocrocite, and akaganeite and Fe(II, III)(hydr)oxides (average oxidation number 2.67) green rust (carbonate) and green rust (sulfate). 


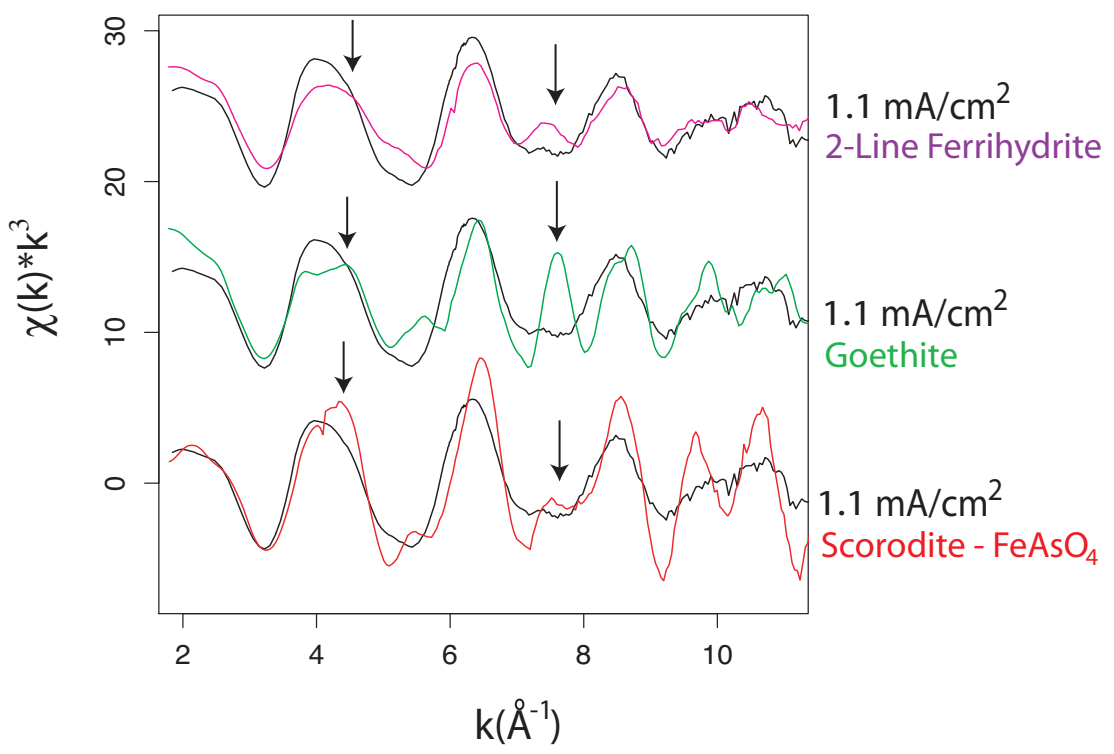

Figure 4.6: Iron EXAFS spectra (weighted by $\mathrm{k}^{3}$ ) for EGA generated at $j=1.1 \mathrm{~mA} / \mathrm{cm}^{2}$ compared to reference spectra for iron (hydr)oxides 2-line ferrihydrite (2L FH), goethite, and scorodite. Arrows note significant differences between spectra. 
and lepidocrocite. The four spectra in Figure 4.6 are similar in line-shape, phase, and amplitude characteristics. As 2L FH, goethite, and scorodite all have Fe[III]-O octahedra as the basic structural unit, one may infer that the EGA iron (hydr)oxide also has this basic structural unit based on the spectral similarities. The spectra also possess significant spectral differences, noted by arrows. Differences in line shape indicate that the way in which the Fe[III]-O octahedra are linked together (Toner et al., 2008). Recall from Section 2.3 that the basic polyhedra may be joined by faces, edges, or corners, each with a characteristic bond length between Fe-Fe atoms affecting the shape of EXAFS. The differences noted in Figure 4.6 indicate that these polyhedral linkages differ between the four reference spectra and the EGA sample.

The overall similarities between the EGA sample structure and 2L FH compared to other reference spectra suggest that EGA is also a Fe[III] oxyhydroxide, more similar in structure to $2 \mathrm{~L} \mathrm{FH}$ that either goethite or scorodite. The strong feature noted by an

arrow at $\mathrm{k}=7.8 \AA^{-1}$ tends to flatten as 3D structure is lost (Toner et al., 2008) (this is bourne out in the significant decrease in this feature between goethite and $2 \mathrm{~L} \mathrm{FH}$, associated with a decrease in $3 \mathrm{D}$ order). This feature is further diminished in the EGA sample compared to $2 \mathrm{~L} \mathrm{FH,} \mathrm{suggesting} \mathrm{that} \mathrm{the} \mathrm{EGA} \mathrm{may} \mathrm{be} \mathrm{less} \mathrm{ordered} \mathrm{than} 2 \mathrm{~L} \mathrm{FH}$.

\subsubsection{Arsenic bonding structure - comparison to known arsenic-iron complexes}

In Figure 4.7, the $\mathrm{k}^{3}$-weighted As EXAFS spectrum for arsenic-laden EGA generated at $j=1.1 \mathrm{~mA} / \mathrm{cm}^{2}$ is compared to reference spectra for As[V] adsorbed onto 




Figure 4.7: Arsenic EXAFS spectrum (weighted by $\mathrm{k}^{3}$ ) for arsenic-laden EGA generated at $j=1.1 \mathrm{~mA} / \mathrm{cm}^{2}$ compared to reference spectra for $\mathrm{As}[\mathrm{V}]$ adsorbed onto goethtite, $\mathrm{As}[\mathrm{V}]$ adsorbed onto 2-line ferrihydrite $(2 \mathrm{~L} \mathrm{FH})$, and the mineral scorodite $\left(\mathrm{FeAsO}_{4}\right)$. Arrows indicate spectral differences. 
goethite, $\mathrm{As}[\mathrm{V}]$ adsorbed onto 2-line ferrihydrite (2L FH), and the arsenic-iron mineral scorodite $\left(\mathrm{FeAsO}_{4}\right)$. The EGA sample is extremely similar in line shape, phase, and amplitude characteristics both to As[V] adsorbed to goethite and As[V] adsorbed to $2 \mathrm{~L}$ FH. Although similar to scorodite, there are two key difference in line shape, indicated by arrows. Differences in line shape are indicative of differences in the 3D arrangement of atoms. The differences between scorodite and the EGA sample are severe enough to imply that the majority of the arsenic in the sample is not forming scorodite. Based on similarities with the top two reference spectra, the EGA sample is consistent with As[V] adsorbed onto a Fe[III] oxyhydroxide mineral.

\subsubsection{EGA iron structure - comparison between current densities}

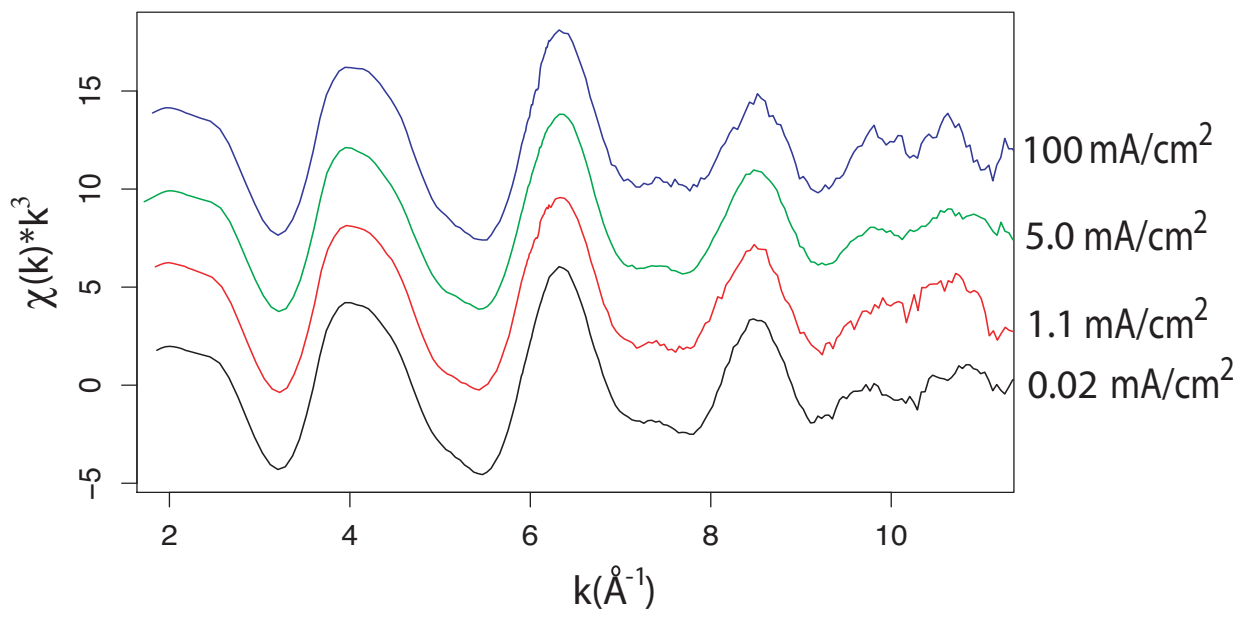

Figure 4.8: Iron EXAFS spectra (weighted by $\mathrm{k}^{3}$ ) for EGA generated at current densities $j=100 \mathrm{~mA} / \mathrm{cm}^{2}, 5.0 \mathrm{~mA} / \mathrm{cm}^{2}, 1.1 \mathrm{~mA} / \mathrm{cm}^{2}$, and $0.02 \mathrm{~mA} / \mathrm{cm}^{2}$ (displayed top to bottom).

In Figure 4.8, the $\mathrm{k}^{3}$-weighted Fe EXAFS spectrum for EGA generated at 
current densities $j=100 \mathrm{~mA} / \mathrm{cm}^{2}, 5.0 \mathrm{~mA} / \mathrm{cm}^{2}, 1.1 \mathrm{~mA} / \mathrm{cm}^{2}$, and $0.02 \mathrm{~mA} / \mathrm{cm}^{2}$ are compared. The four spectra are all extremely similar in line shape, phase, and amplitude. Some small differences exist in the amplitude near $\mathrm{k}=10 \AA^{-1}$, but it is difficult to attribute this to a change in structure because of the higher noise at high $\mathrm{k}$, especially in these spectra (see Section 4.2.4). In all other ways, these spectra are consistent with the same 3D iron (hydr)oxide structure across all four current densities.

Significantly, this result is inconsistent with the hypothesis that the higher arsenic removal capacity demonstrated at $j=0.02 \mathrm{~mA} / \mathrm{cm}^{2}$ compared to $j=1.1 \mathrm{~mA} / \mathrm{cm}^{2}$ (Section 3.3.4) is due to the increased presence of an iron (hydr)oxide with a higher affinity for arsenic in the EGA. The EXAFS signal is averaged over all of the iron (hydr)oxides present in EGA, and any significant differences in structure in the primary component would appear in the EXFAS spectrum. An alternative hypothesis is discussed more in Section 4.3.10 below.

Recall that from comparisons to iron reference compounds, EGA at $j=1.1 \mathrm{~mA} / \mathrm{cm}^{2}$ was found to be consistent with a Fe[III] oxyhydroxide similar in structure to 2-line ferrihydrite (Section 4.3.6). Given the similarity in EGA structure across current density shown here, EGA at $j=0.02 \mathrm{~mA} / \mathrm{cm}^{2}$ is also consistent with an Fe[III] oxyhydroxide, suggesting that EGA at $0.02 \mathrm{~mA} / \mathrm{cm}^{2}$ is Fe[III] based rather than Fe[II] based. Similar results were obtained from Fe XANES spectra above (Section 4.3.5). The provides further evidence against the hypothesis suggested by polarization studies that EGA at $j=$ $0.02 \mathrm{~mA} / \mathrm{cm}^{2}$ is primarily Fe[II]-based (Section 3.3 .10 ) 


\subsubsection{Arsenic bonding structure - comparison between current densi- ties}

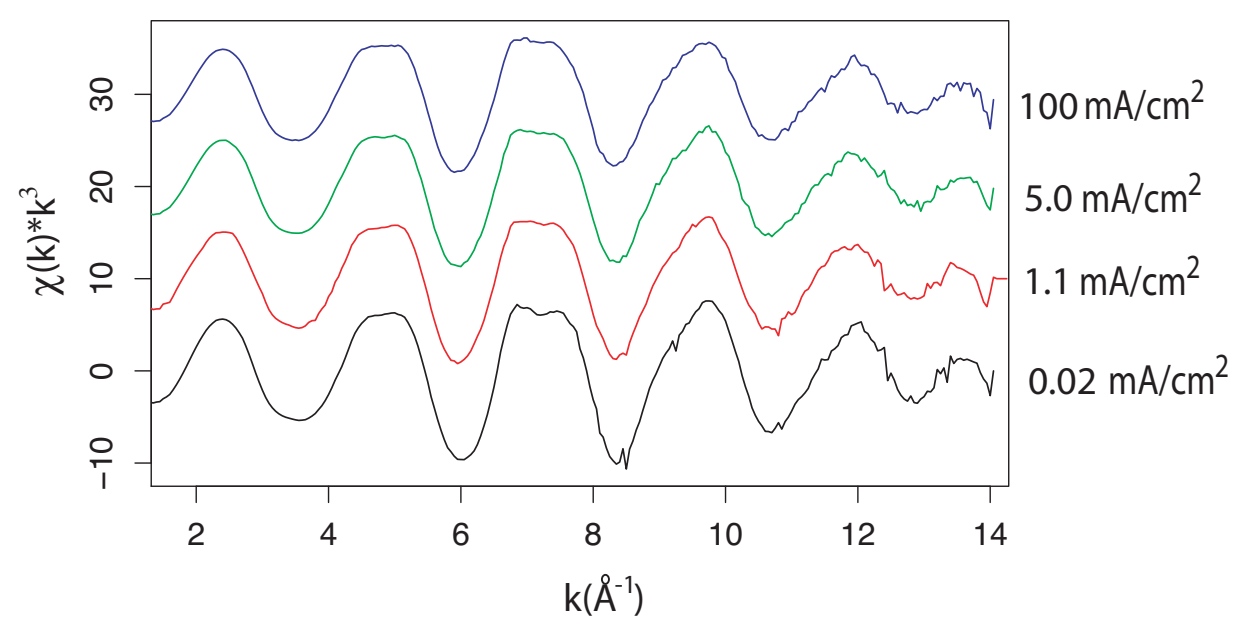

Figure 4.9: Arsenic EXAFS spectra (weighted by $\mathrm{k}^{3}$ ) for EGA generated at current densities $j=100 \mathrm{~mA} / \mathrm{cm}^{2}, 5.0 \mathrm{~mA} / \mathrm{cm}^{2}, 1.1 \mathrm{~mA} / \mathrm{cm}^{2}$, and $0.02 \mathrm{~mA} / \mathrm{cm}^{2}$ (displayed top to bottom).

In Figure 4.9, the $\mathrm{k}^{3}$-weighted As EXAFS spectrum for EGA generated at current densities $j=100 \mathrm{~mA} / \mathrm{cm}^{2}, 5.0 \mathrm{~mA} / \mathrm{cm}^{2}, 1.1 \mathrm{~mA} / \mathrm{cm}^{2}$, and $0.02 \mathrm{~mA} / \mathrm{cm}^{2}$ are compared. As was the case for Fe EXAFS, the line shape, phase, and amplitude across current densities is extremely similar. This suggests that the arsenic bonding structure, as well as the underlying structure of EGA bound to arsenic, is the same across current densities. Given the comparison of EGA at $j=1.1 \mathrm{~mA} / \mathrm{cm}^{2}$ to arsenic reference spectra above (Section 4.3.7), this suggests that the predominant bonding structure responsible for arsenic removal at all current densities is arsenic adsorption onto a Fe[III] oxyhydroxide mineral. 


\subsubsection{Arsenic capture in the bulk solution compared to the electrode surface}

During ECAR treatment, iron (hydr)oxides are generated on the surface of the electrode which may be very different in composition from the EGA generated in the bulk solution. Arsenic may be captured on either the electrode surface or in the bulk. As described in Section 4.2.4.3, several failed attempts were made to detect an arsenic XAS signal from electrode surface layer samples scraped from the iron anode immediately after ECAR treatment at $j=1.1$ and $5.0 \mathrm{~mA} / \mathrm{cm}^{2}$. This implies that most of the arsenic removal in ECAR occurs in the bulk rather than on the electrode surface at current densities $j=1.1$ and $5.0 \mathrm{~mA} / \mathrm{cm}^{2}$. This is consistent with results showing a much higher As/Fe mass ratio in EGA sludge compared to electrode scrapings at $j=$ $0.02 \mathrm{~mA} / \mathrm{cm}^{2}$ (Section 4.3.4). Interestingly however, the electrode scrapings at $j=0.02$ $\mathrm{mA} / \mathrm{cm}^{2}$ did show a strong arsenic XAS signal during the same XAS run and under the same beam conditions as described above. The null signal at $j=1.1$ and $5.0 \mathrm{~mA} / \mathrm{cm}^{2}$ suggests that chemical analysis of these electrode scrapings would yield an even lower (if existent) As/Fe ratio than the electrode scrapings at $j=0.02 \mathrm{~mA} / \mathrm{cm}^{2}$.

The presence of arsenic on the electrode surface at $j=0.02 \mathrm{~mA} / \mathrm{cm}^{2}$ compared

to no arsenic on the electrode surface at $j=1.1$ and $5.0 \mathrm{~mA} / \mathrm{cm}^{2}$ suggests an alternative hypothesis for increased removal capacity at $j=0.02 \mathrm{~mA} / \mathrm{cm}^{2}$. Perhaps at low current densities, more arsenic is adsorbed to the electrode surface layer, increasing removal capacity. This is further suggested by the fact that, during Matrix 2 batch tests (Chapter 3), the electrode-surface-area to active-solution-volume ratio $(\mathrm{A} / \mathrm{V})$ was allowed to 
vary to accommodate the factor of 5000 difference in current density between $j=0.02$ and $100 \mathrm{~mA} / \mathrm{cm}^{2}$. This resulted in a factor of 16 increase in electrode area relative to the treatment volume for $j=0.02 \mathrm{~mA} / \mathrm{cm}^{2}$ compared to $j=100 \mathrm{~mA} / \mathrm{cm}^{2}$. Further study is planned to explore this alternative hypothesis.

\subsection{Chapter Summary}

The reaction products of ECAR (known as EGA sludge) have been analyzed using SEM imaging, XRF mapping, chemical analysis, and X-ray absorption spectroscopy (EXAFS and XANES). SEM images showed that EGA is very homogenous and made up of rounded popcorn-like particles with a primary aggregate size of 50-100 nm. Arsenic breakthrough experiments with increasing pore size showed that the cluster size of EGA is as least $800 \mathrm{~nm}$. XRF mapping of the EGA sludge confirmed homogeneity and showed that arsenic is evenly captured across EGA.

Arsenic-laden EGA sludge is a waste product of ECAR and must be chemically characterized for safe disposal. An initial chemical characterization (including only Fe and As) showed that the waste from a representative ECAR treatment of synthetic Bangladesh groundwater $\left(j=1.1 \mathrm{~mA} / \mathrm{cm}^{2}, q=175 \mathrm{C} / \mathrm{L}\right.$, initial arsenic $=590 \mu \mathrm{g} / \mathrm{L}$, final arsenic $=5.0 \mu \mathrm{g} / \mathrm{L}, \mathrm{SBGW}-2$ recipe) contained $3300 \mathrm{ppm}$ arsenic by mass with an As/Fe mass ratio of 0.0076 . Estimates of the expected amount of waste per treated liter based on the amount of arsenic removed from solution at $q=150 \mathrm{C} / \mathrm{L}$ were a factor of 3 higher than the measured amount of waste produced. This indicates that more study is needed for accurate waste estimates. 
Fe XANES spectra for EGA generated at $j=0.02-100 \mathrm{~mA} / \mathrm{cm}^{2}$ were consistent with an iron oxidation state of $3+$ at all current densities. This disproved the hypothesis that EGA generated at $j=0.02 \mathrm{~mA} / \mathrm{cm}^{2}$ is dominated by Fe[II]-based (hydr)oxides, as suggested by polarization curves in Section 3.3.10.

Comparisons between Fe EXAFS spectra of EGA sludge generated at current densities $j=0.02,1.1,5.0$, and $100 \mathrm{~mA} / \mathrm{cm}^{2}$ revealed no discernible difference in iron (hydr)oxide structure with current density. Comparisons between arsenic EXAFS spectra similarly revealed no discernible difference in the structure of the iron (hydr)oxide bound to arsenic with current density. These results are inconsistent with the hypothesis that differences in EGA composition with current density account for the differences in arsenic removal capacity with current density measured in Chapter 3. An alternative hypothesis was formulated that attributes the increase in arsenic removal capacity to an increase in arsenic capture by the electrode surface layer at low current density. This could potentially be accounted for by the increase in the electrode-surface-area to active-solution-volume ratio $(\mathrm{A} / \mathrm{V})$ used in batch tests to accommodate the factor of 5000 difference in current density between $j=0.02$ and $100 \mathrm{~mA} / \mathrm{cm}^{2}$. Further experiments are planned to test this hypothesis.

Based on Fe EXAFS spectral comparisons between EGA sludge and reference iron (hydr)oxide compounds, EGA sludge was found to be consistent with a Fe[III] oxyhydroxide similar in structure to 2-line ferrihydrite. This is consistent with the Fe XANES results showing a $3+$ oxidation state for iron.

Comparisons were made between As EXAFS spectra of EGA sludge and refer- 
ence compounds, including arsenate adsorbed to 2-line ferrihydrite, arsenate adsorbed to goethite, and the As-Fe mineral phase scorodite $\left(\mathrm{FeAsO}_{4}\right)$. Based on strong similarities in key spectral features such as line shape, phase, and amplitude, the arsenic bonding structure responsible for arsenic removal in ECAR treatment was found to be very consistent with arsenate adsorbed to a Fe[III] oxyhydroxide. Based on key differences in line shape, the spectrum is inconsistent with scorodite. Further analysis is underway to determine the bonding structure from characteristic bond lengths determined by shell-by-shell fits to the EXAFS spectra.

Combining these results, two important conclusions are reached - (1) there is no significant difference in the arsenic removal mechanism of ECAR during operation at different current densities (at least in the bulk solution), and (2) based on EXAFS spectral comparisons, the arsenic removal mechanism in ECAR is consistent with arsenate adsorption onto a homogenous Fe[III] oxyhydroxide similar in structure to 2-line ferrihydrite. Further analysis of the EXAFS spectra beyond the scope of this thesis will allow for more concrete conclusions. 


\section{Chapter 5}

\section{ECAR Performance in Real}

\section{Bangladesh Groundwater}

\subsection{Introduction}

It is difficult to model the diverse and complex chemistry state of real groundwater. Although care was taken to build a synthetic groundwater using the average ion concentrations found in Bangladesh, actual groundwater in Bangladesh covers a wide range of concentrations of ions as well as composition of ions (i.e. different relative concentrations) (BGS, 2001). It is known, for example, that bicarbonate, phosphate, and silicate can adversely affect arsenic adsorption onto iron (hydr)oxides (Section 2.4.6) and that these ions exist in relatively high concentrations in the groundwater of Bangladesh (Section 3.2.3). The presence of these ions together can interfere more than the sum of each appearing alone (Meng et al., 2002), making it difficult to predict the relative 
and absolute concentrations that will lead to a greater or lesser effect. In addition, the ions in real groundwater have been interacting for much longer than in synthetic water, and may have aged into compounds that cannot easily be mimicked in the synthetic groundwater. Thus, it is essential to demonstrate the ability of ECAR to remove arsenic from real arsenic-contaminated groundwater from tube well sources in Bangladesh.

In addition, observations of synthetic groundwater designed to mimic conditions in Bangladesh (described in Section 3.2.3) showed almost complete oxidation of As[III] to $\mathrm{As}[\mathrm{V}]$ within 2 days. Iron (hydr)oxides are known to have a lower affinity for As[III], which is also known to sorb at a slower rate (Bissen \& Frimmel, 2003). During ECAR treatment, there should be a net oxidation of $\mathrm{As}[\mathrm{III}]$ to $\mathrm{As}[\mathrm{V}]$ in the electrochemical cell (Section 2.5.3), however the rate of this net reaction is not known, and there is some suggestion that this may be the rate determining step for the residence time $t_{r}$ (Section 3.3.9). It was also observed the that the residual arsenic after treatment is $>80 \%$ As[III] (Section 3.3.9). Thus it is prudent to explore if similar oxidation is seen in real Bangladesh groundwater removed from a tube well. If oxidation does occur, a waiting period before treatment could potentially increase the efficiency of arsenic removal.

\subsubsection{Issues of groundwater transport and storage}

Due to laboratory and time constraints in Bangladesh, it was decided to transport groundwater samples from tube wells in Bangladesh to the lab in Berkeley, CA for ECAR testing. Transport occurred within three weeks of sample collection. The 
transport of water inevitably changes certain water qualities, making it important to list and understand the potential changes.

Based on observations of synthetic groundwater in the lab showing rapid oxidation of $\mathrm{As}[\mathrm{III}]$ to $\mathrm{As}[\mathrm{V}]$, it is likely that most or all of the As[III] originally present in real groundwater will be oxidized to $\mathrm{As}[\mathrm{V}]$ by the time of testing. Gallagher et al. (2004) reported changes in the ratio of $\mathrm{As}[\mathrm{III}]$ to $\mathrm{As}_{\text {tot }}$ in groundwater shipped overnight without treatment. Batch tests of ECAR using synthetic groundwater showed that $80 \%$ of the residual aqueous arsenic after treatment was As[III] (Section 3.2.1), implying that ECAR generated iron (hydr)oxides have a higher affinity for As[V]. A reduction in the amount of As[III] relative to As[V] could therefore artificially decrease the apparent required dosage for complete arsenic removal in real Bangladesh groundwater.

Arsenic preservation problems in iron-rich water samples have been reported by Aggett \& Kriegman (1987), Borho \& Wilderer (1997), Anderson et al. (1986), and Boyle et al. (1998). Arsenic-contaminated groundwater in Bangladesh is known to contain iron (average $\mathrm{Fe}_{\text {tot }}=5.6 \pm 5.9 \mathrm{mg} / \mathrm{L}$ according to National Survey data with $\mathrm{As}_{\text {tot }}>10$ $\mu \mathrm{g} / \mathrm{L}$ in BGS (2001)). Iron(II) is abundant in well waters, and is slowly oxidized by oxygen in air, forming iron[III]hydroxides which precipitate out of the water (Crisp \& Chowdhury, 2001). Calcium and bicarbonate are also abundant in well waters, including those of Bangladesh. When exposed to air, carbon dioxide is lost and calcium carbonate precipitates (Crisp \& Chowdhury, 2001). Aqueous arsenic adsorbs to iron(III) hydroxide and calcium carbonate, reducing the aqueous arsenic concentration in the water sample. Mamtaz \& Bache (2001) found that naturally occurring iron $\geq 1.1 \mathrm{mg} / \mathrm{L}$ in Bangladesh 
groundwater could be used to reduce $100 \mu \mathrm{g} / \mathrm{L}$ As[III] to below $50 \mu \mathrm{g} / \mathrm{L}$ simply by mixing the solution and allowing it to settle for 3 days. Similar processes will occur in the samples as iron precipitates and settles, or sticks to the bottle sides - reducing the arsenic concentration in the supernatant of the sample bottle from the original arsenic concentration in the tube well water. Gallagher et al. (2004) reported losing 25-40\% of total arsenic during overnight transport in US groundwater samples containing iron precipitate. Any loss in arsenic due to iron precipitation in the sample must be accounted for by measuring the arsenic concentration in the supernatant immediately before testing. This will not prevent arsenic loss, but will allow for an accurate understanding of the arsenic removed due to ECAR treatment as opposed to iron precipitation during storage.

\subsubsection{Research objectives}

Specific research objectives for real Bangladesh groundwater included the following:

1. Demonstrate the ability of ECAR treatment to reduce arsenic levels from $100-500$ $\mu \mathrm{g} / \mathrm{L}$ in real Bangladesh groundwater to below either the Bangladesh maximum allowable limit $(50 \mu \mathrm{g} / \mathrm{L})$ or ideally, the WHO recommended maximum limit (10 $\mu \mathrm{g} / \mathrm{L})$

2. Measure the level of As[III] to As[V] oxidation in tube well water samples up to seven days after collection.

3. Characterize the co-occurring solute composition of tube well water samples and compare to regional and national survey data from BGS (2001). 


\subsection{Methods}

\subsubsection{Arsenic analysis and arsenic speciation}

All reported arsenic concentrations were measured using ICPMS provided by Curtis \& Tompkins, Ltd (as described in Section 3.2.1) unless otherwise noted. Samples for ICPMS were transported to Berkeley in plastic $15-\mathrm{mL}$ vials that had been radiation sterilized by the vendor (BD Biosciences). Preservation and digestion of samples occurred in Berkeley, and in every case digested samples appeared clear and free of precipitate. Quick Test arsenic measurements, as described in Section 3.2.1, were sometimes used in the field and lab as a rough estimate of arsenic concentration. Separation of As[III] from $\mathrm{As}[\mathrm{V}]$ in the field was performed using arsenic speciation cartridges described in Section 3.2.1. Experiment specific procedures used in the field are described below.

\subsubsection{Tube well water sample collection and storage}

From March 23 to April 7, 2007, thirteen tube wells in Bangladesh were sampled, twelve with high arsenic concentrations $\left(\mathrm{As}_{t o t}>100 \mu \mathrm{g} / \mathrm{L}\right)$. Samples were collected from five villages in Jhikargachha Upazila and Abhaynagar Upazila (both of Jessore district in Khulna division) and one village from Sonargaon Upazila, just outside of Dhaka (see Figure 5.1). Seven samples were later used in testing, listed in Table 5.1, including at least one sample from each village vistited.

Villages containing tube wells with high levels of arsenic were identified by the Bangladesh Rural Advancement Committee (BRAC), a Bangladeshi NGO, using the final report of their 1999-2000 arsenic study (BRAC, 2000). During all field visits we 


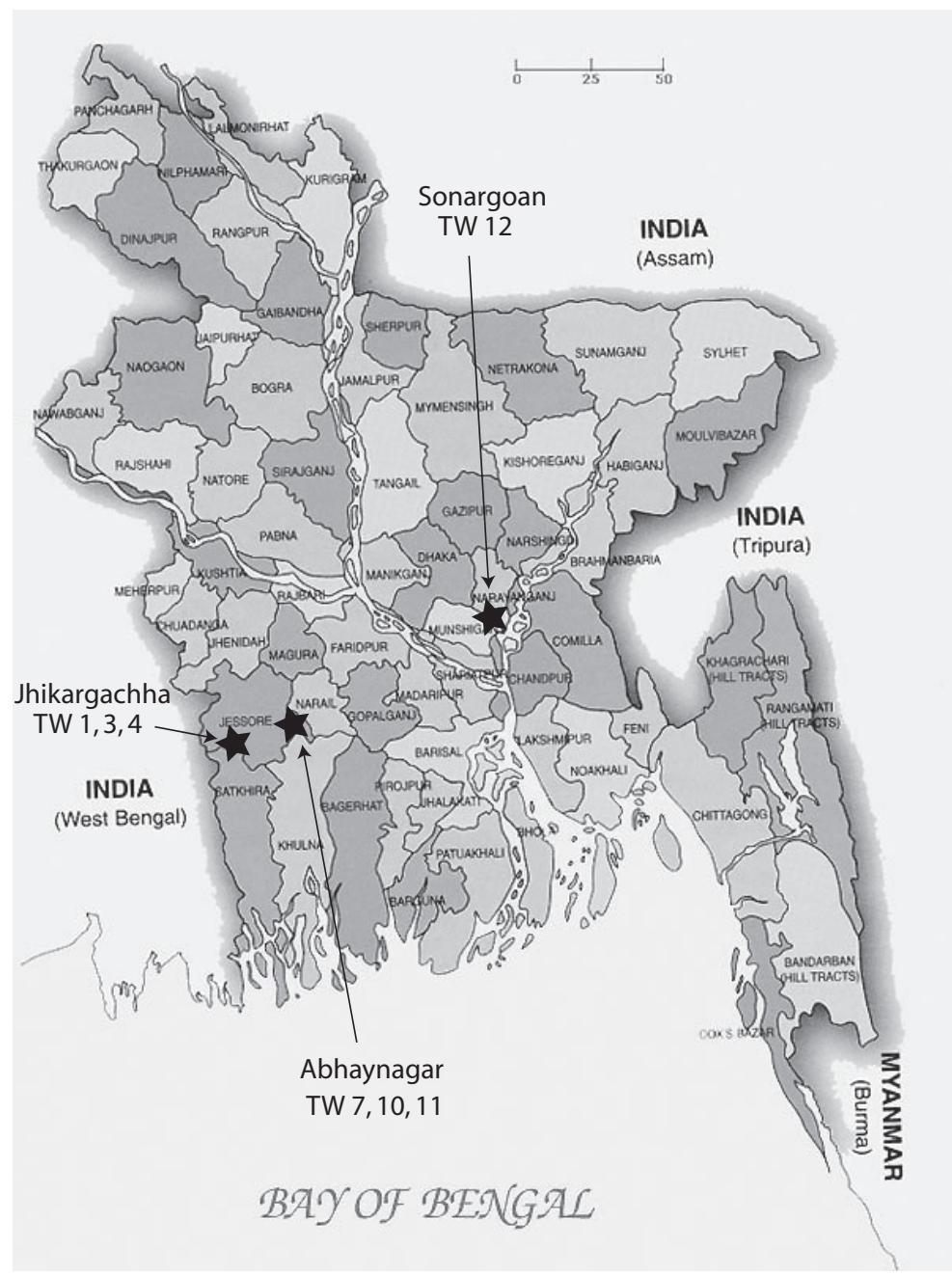

Figure 5.1: Map of Bangladesh highlighting areas visited. Map modified from www.homelandbangladesh.com. 
were accompanied by at least one BRAC employee. In each village, BRAC employees, community leaders, and local villagers helped identify households with contaminated tube wells.

At each tube well, the initial pH, Dissolved Oxygen (DO), and arsenic concentration were measured. The $\mathrm{pH}$ and DO were measured twice in the field and averaged, using a portable $\mathrm{pH}$ and DO probe and meter (SympHony). The $\mathrm{pH}$ probe was disconnected and dried for travel to Bangladesh. Upon arrival, it was rinsed in tap water and allowed to sit in electrolyte solution for $60 \mathrm{~min}$. The $\mathrm{pH}$ probe was calibrated every other day using $\mathrm{pH} 7.01$ and $\mathrm{pH} 4.01$ buffer solutions (Orion) which were stored in a cool dry place. The DO probe was calibrated daily using a wet sponge (same procedure described in 3.2.2). The arsenic concentration was estimated on site using the QuickTest. The goal of the initial measurement was only to accept or reject the well as arsenic-contaminated, thus the water was not initially diluted to be within the optimum measurement range of the test, leading to initial results with high errors (up to 200\%). For later samples, 20x dilutions were made using Jessore city tap water pre-filtered to remove particulates (via an MSR hand-pumped water filter built for hiking) and subsequently determined by ICPMS to contain $<1 \mu \mathrm{g} / \mathrm{L}$ arsenic.

If arsenic levels were sufficiently high according to the Quick Test (in most cases, greater than $200 \mu \mathrm{g} / \mathrm{L})$, two or three 1-liter tube well water samples were collected. All samples were collected after pumping the tube well for five minutes in order to ensure that the sample would be free from biological contamination and oxygenated water from the 
tube well column ${ }^{1}$. All sample bottles (previously unused 1L high density polyethylene) were filled to the brim and tightly capped to reduce exposure to oxygen. All sample bottles were stored in the dark (to prevent algae growth and photo-oxidation) and not opened again until the first testing was performed, either in Bangladesh (to monitor As $[\mathrm{III}]$ to As[V] oxidation) or in Berkeley (ECAR treatment and measurement of cooccuring solute concentrations). No acid or preservatives were added to the tube well samples. Tube well samples are designated by TW $\mathrm{x}$, where " $\mathrm{x}$ " numbers the tube wells in order of sample collection.

Aliquots for ICPMS measurement of the initial arsenic concentration were removed from one sample bottle for each tube well within 0-9 hours of collection (samples times listed in Table 5.1). In retrospect, initial water samples should have all been collected at the tube well itself and preserved with nitric acid to avoid arsenic adsorption to natural iron hydroxides precipitating in the sample bottle (see Section 5.1.1). It was noted that within 1-2 hours of collection some of the plastic sample bottles containing tube well water began to turn brown, with the exception of TW 12 from Sonargaon Upazila. In addition, a small amount of brown precipitate began to form in the bottom of the bottles, though TW 12 samples remained clear. The brown precipitate and bottle discoloration indicate the precipitation of iron hydroxides, likely removing some of the original arsenic present in the tube well water via adsorption and settling. Thus the initial arsenic concentration listed in Table 5.1 is a lower limit of the arsenic present in the tube well at the time of pumping.

\footnotetext{
${ }^{1}$ Tube well depth is typically 40-80 m (Burgess \& Ahmed, 2006).
} 
Table 5.1: Tube well locations, initial arsenic concentration and time of initial aliquot, and list of tests performed on each tube well water sample.

\begin{tabular}{|c|c|c|c|c|c|c|}
\hline Tube Well (TW) & District & Upazila $^{\mathrm{a}}$ & Union & Tests b & $\begin{array}{c}\text { Initial } \\
\text { As } \\
(\mu \mathrm{g} / \mathrm{L})\end{array}$ & $\begin{array}{c}\text { Time of } \\
\text { As Sample } \\
\text { (Hrs) }\end{array}$ \\
\hline 1 & Jessore & Jhikargachha & Godkhali & $I$ & 667 & 9.0 \\
\hline 3 & Jessore & Jhikargachha & Godkhali & $A$ & 444 & 7.5 \\
\hline 4 & Jessore & Jhikargachha & Godkhali & $A, E$ & 333 & 6.5 \\
\hline 7 & Jessore & Abhaynagar & Prembug & $I$ & 378 & 0.7 \\
\hline 10 & Jessore & Abhaynagar & Prembug & $E$ & 378 & 2.7 \\
\hline 11 & Jessore & Abhaynagar & Prembug & $E$ & 356 & 2.1 \\
\hline 12 & Narayanganj & Sonargoan & Amnipur & $I, A, E$ & 600 & 0 \\
\hline
\end{tabular}

\subsubsection{Measurements of $\mathrm{As}[\mathrm{III}] / \mathrm{As}_{t o t}$ ratio over time}

Three tube wells were chosen arbitrarily for As[III]/Astot monitoring over seven days in the field - TW 3, 4, and 12. In each case, the As[III]concentration was measured by filtering an aliquot of tube well water sample through a single disposable speciation cartridge and storing the effluent for ICPMS arsenic measurement in Berkeley. For TW 4, the tube well water was pre-filtered (via an MGR hand-pumped water filter) to remove high levels of particulate matter before being filtered through the speciation cartridge (as recommended by the manufacturer). The $\mathrm{As}_{t o t}$ concentration was measured by removing an aliquot of tube well water sample and storing it unfiltered for ICPMS arsenic measurement in Berkeley. Samples were not preserved or digested (via addition of $10 \% \mathrm{HNO}_{3}$ ) until 1-2 days before ICPMS testing in Berkeley.

For TW 3 and 4, the initial Astot aliquot was unfortunately removed from the sample 6-8 hours after the As[III] aliquot was filtered. Due to iron precipitation observed 
in the sample after 1-2 hours, the total aqueous arsenic is likely to have decreased during the first 6-8 hours, allowing only an upper limit estimate of the initial As[III]/Astot ratio in the tube well. For later measurements, aliquots for $\mathrm{As}[\mathrm{III}]$ and Astot measurement were removed at the same time. However, due to the iron precipitation, some As[III] was likely removed while attached to iron hydroxide particles during filtration through the speciation cartridge itself. The cartridge is filled with aluminosilicate sand to bind to As[V]. However, if large iron hydroxide particles are passed, it effectively acts as a sand filter. The $\mathrm{As}_{\text {tot }}$ aliquot was not filtered through sand, again preventing a robust comparison between the $\mathrm{As}[\mathrm{III}]$ and $\mathrm{As}_{\text {tot }}$ measured. A lower limit to the $\mathrm{As}[\mathrm{III}] / \mathrm{As}_{t o t}$ ratio might have been determined for the 2-day and 4-day values based on the assumption that As[III] could only decrease due to precipitate filtered out in the speciation cartridge. However, large quantities of precipitate may have changed the ability of the aluminosilicate to remove As $[\mathrm{V}]$ properly. Thus the data from these wells was thrown out, and valuable lessons were learned about field speciation.

For TW 12, all aliquots to measure As[III] and $\mathrm{As}_{\text {tot }}$ were removed at the same time. Iron content appeared to be very low in TW 12 samples, evidenced by no visible iron precipitate after 20 days and only a very slight browning of the sample bottle. Measurements of $\mathrm{As}_{\text {tot }}$ taken from the sample bottle demonstrate a decrease in $\mathrm{As}_{\text {tot }}$ of only $15 \%$ after 8 days of storage (Table 5.3), also suggesting low iron precipitation. Finally, ICPMS measurement of the total iron concentration in 11 day old TW 12 samples showed $0.1 \mathrm{mg} / \mathrm{L}$ (Table 5.4). Low iron precipitation will minimize the effect of As[III] removal in speciation cartridges for TW 12. For comparison, the decrease in aqueous 
Astot measured in the sample for TW 4, which contained visible iron precipitate, was $72 \%$ over 13 days (Table 5.3 ).

\subsubsection{Chemical analysis for co-occuring solutes}

One tube well water sample from each Upazila (identified in Table 5.1) was tested for a series of co-occuring solutes (i.e. background ion composition) to determine how representative the samples were of wells in the BGS. Samples were tested at Curtis \& Tomkins Laboratory (Berkeley, CA) for $\mathrm{Cl}^{-}, \mathrm{NO}_{3}^{-}$and $\mathrm{SO}_{4}^{2-}$ using ion chromatography (EPA procedure 300.0), $\mathrm{P}_{\text {tot }}$ (EPA procedure 365.2), and $\mathrm{Ca}, \mathrm{Mg}, \mathrm{Na}, \mathrm{K}, \mathrm{Fe}$, and $\mathrm{Mn}$ using ICPMS (procedure EPA 6020). Between 11-19 days after collection, samples were shaken in the original sample bottle before aliquots of $100-150 \mathrm{~mL}$ were removed for solute testing. This is the same procedure used before aliquots were removed for ECAR testing after aging approximately the same amount of time (described in Section 5.2.5), so the measured solute concentrations are likely to represent the aqueous concentrations of solutes present in aliquots tested with ECAR. Unfortunately, the tube well water samples were not preserved properly to measure alkalinity or $\mathrm{HCO}_{3}^{-}$concentration at the time of ECAR testing, and no equipment or lab was available and within our budget capable of measuring $\mathrm{Si}$ or silicate at the time. In the future, it would be useful to obtain pre-ECAR measurements of these solutes, given the detrimental effect they may have on arsenic removal via ECAR (Section 2.4.6). 


\subsubsection{ECAR Treatment}

Sealed 1-liter tube well water samples were opened in Berkeley 8-22 days after collection (specific dates and tube wells listed in Table 5.3). Dissolved oxygen (DO) was measured immediately after opening, with the change in DO from the time of collection presented in Table 5.3. A DO rise of $<2.5 \mathrm{mg} / \mathrm{L}$ in most cases indicates minimal oxygen contamination during transport and storage of samples. In the case of TW 11, oxygen contamination was the highest $(\Delta \mathrm{DO}=4.16 \mathrm{mg} / \mathrm{L})$ but the sample still had not reached equilibrium with the atmosphere $(\mathrm{DO}=7-9 \mathrm{mg} / \mathrm{L})$. Sample $\mathrm{pH}$ changed very little during transport and storage (average $p H-p H_{\text {collection }}=-0.08$, maximum $\left.p H-p H_{\text {collection }}=-0.39\right)$. Four tube well water samples $(\mathrm{TW} 4,10,11$ and 12) were chosen to cover a range of initial arsenic concentrations as well as geographic locations (listed in Table 5.1). Batch tests were performed on $850 \mathrm{~mL}$ aliquots of sample, decanted from the sample bottle after vigorous shaking, with the exception of TW 4 which was not shaken. ECAR procedures as described in Section 3.2.5 were used with the 850 mL-separated cathode cell (described in Section 3.2.4). Iron electrodes were rinsed in dilute $\mathrm{HCl}$ followed by deionized water before each test. Current densities of $0.75-1.1$ $\mathrm{mA} / \mathrm{cm}^{2}$ were applied for a residence time of $t_{r}=11-22$ minutes $(q=86-175 \mathrm{C} / \mathrm{L}$; see Table 5.3 for the exact operating conditions used for each well). For comparison, one batch test using SBGW-1 synthetic groundwater $\left(\mathrm{As}_{t o t}=530 \mu \mathrm{g} / \mathrm{L}, \mathrm{As}[\mathrm{III}] / \mathrm{As}_{t o t}=\right.$ $0.58, \mathrm{pH}=7.41, \mathrm{DO}=1.27 \mathrm{mg} / \mathrm{L}$, background ions described in Table 3.1$)$ was dosed concurrently using the same $\operatorname{setup}\left(j=1.1 \mathrm{~mA} / \mathrm{cm}^{2}, t_{r}=22 \mathrm{~min}, q=179.8 \mathrm{C} / \mathrm{L}\right)$.

Since only one ECAR test could be performed per 1-liter tube well water sample, 
the applied dosage was purposefully higher than the minimal dosage required to reduce $600 \mu \mathrm{g} / \mathrm{L}$ to $<10 \mu \mathrm{g} / \mathrm{L}$ using SBGW-1 synthetic groundwater. Higher dosages were used to remediate higher initial arsenic concentrations (dosage based on Quick Test measurement of initial arsenic). The target current density for all tests was $1.1 \mathrm{~mA} / \mathrm{cm}^{2}$, however, lower current densities were used for water with a high resistivity so as not to overload the Galvonostat at $1.1 \mathrm{~mA} / \mathrm{cm}^{2}$. An alternative would have been to bring the electrodes closer together, however an overload could not be discovered until treatment was already underway, and a delay during dosage could have produced unknown side effects that would be difficult to account for.

Dosed samples were mixed (as described in Section 3.2.5) with aliquots were removed every 15 minutes starting at $t_{M}=0$ min followed by filtration (as described in Section 3.2.5) and a Quick Test for arsenic. When the Quick Test measured $\mathrm{As}_{\text {tot }}=0$ $\mu \mathrm{g} / \mathrm{L}$ for two subsequent measurements, the mixing was stopped and the final filtrate was sent for ICPMS arsenic testing. This resulted in $t_{M}=15-30$ min. Aliquots filtered after $t_{M}=0$ min were sent for ICPMS testing as well. For TW 4, ICPMS measurements were made at $t_{M}=0,15,30,45$, and 60 min to verify the Quick Test results showing $\mathrm{As}_{\text {tot }}=0$ $\mu \mathrm{g} / \mathrm{L}$ for successive measurements. Note that the filtration process takes 3-4 minutes per aliquot, during which time the remaining solution is mixed as it flows through the filter. The mixing time $t_{M}$ refers to the start of filtration. Thus the mixing time has an error of $\approx+3 \min$ in each case.

As noted in Section 3.2.1, each set of samples sent to C\&T for ICPMS testing was accompanied by a blank sample, containing only deionized (DI) water and 10\% 
$\mathrm{HNO}_{3}$ as a preservative. Usually, no arsenic was detected in blank samples. However, the blank sample sent in with samples from TW 4 and TW 11 was reported to have $10 \mu \mathrm{g} / \mathrm{L}$. A second blank sample was made using the same bottle of $\mathrm{HNO}_{3}$ used to preserve TW 4 and TW 11 along with a blank sample using fresh $\mathrm{HNO}_{3}$. The fresh $\mathrm{HNO}_{3}$ sample showed no arsenic (as expected), while the sample with acid used for TW 4 and TW 11 was reported to have $18 \mu \mathrm{g} / \mathrm{L}$, pointing to arsenic contamination in the acid bottle. ECAR tests could not be repeated, since the majority of tube well water was used up for a single batch test. In the end, a systematic error of $10 \mu \mathrm{g} / \mathrm{L}$ was subtracted from TW 4 and TW 11 measurements, choosing the more conservative arsenic value measured in the blanks. In addition to the systematic error, the ICPMS measured value for TW 4 after $t_{M}=0$ min came out to be $170 \mu \mathrm{g} / \mathrm{L}$ - much higher than the initial arsenic measured before the ECAR testing, Asinit $=103 \mu \mathrm{g} / \mathrm{L}$, and too high to be explained by a systematic error of $10-20 \mu \mathrm{g} / \mathrm{L}$. Contamination during dosage is possible from electrode use in previous batch tests, however contamination was not seen elsewhere and all electrodes were washed in $\mathrm{HCl}$ before testing, making contamination unlikely. Quick Test measurements at $t_{M}=0$ min reported $3 \pm 10 \mu \mathrm{g} / \mathrm{L}$, consistent with other tube wells, but inconsistent with the ICPMS measurement. Given the known acid contamination affecting the ICPMS value, the Quick Test measurement was used for this point only. ICPMS measurements for remaining points were $<15 \mu \mathrm{g} / \mathrm{L}$, and since contamination could never artificially lower arsenic, these could not be affected by as large of an anomaly as the $t_{M}=0 \mathrm{~min}$ point. This experience led to new procedures for adding acid to ICPMS samples, including redundancy to prevent contamination of the 
Table 5.2: $\mathrm{As}_{\text {tot }}$, As[III], and As[III]/Astot ratio for TW 12 up to 7 days after collection.

\begin{tabular}{lccccc}
\hline \hline $\begin{array}{l}\text { Days since } \\
\text { Collection }\end{array}$ & $\begin{array}{c}\text { Astot } \\
(\mu \mathrm{g} / \mathrm{L})\end{array}$ & $\begin{array}{c}\mathrm{As}[\mathrm{III}] \\
(\mu \mathrm{g} / \mathrm{L})\end{array}$ & $\mathrm{As}[\mathrm{III}] /$ Astot & $\mathrm{pH}$ & $\begin{array}{c}\mathrm{DO} \\
(\mathrm{mg} / \mathrm{L})\end{array}$ \\
\hline & & & & & \\
0 & 600 & 578 & 0.96 & 6.93 & 1.78 \\
1.33 & 533 & 444 & 0.83 & 7.02 & 3.92 \\
3.92 & 567 & 60 & 0.11 & 6.98 & 6.13 \\
7.00 & 511 & 27 & 0.052 & 7.61 & 4.15 \\
& & & & & \\
\hline
\end{tabular}

acid, as well as mandatory back-up samples kept without acid for every point.

In addition to ECAR batch tests performed on tube well samples, an aliquot of one sample (TW 10) was simply filtered using a $0.1 \mu \mathrm{m}$ membrane (as described in Section 3.2.5) to obtain a measure of possible arsenic removal via complexation with natural iron precipitates in the water. The TW 10 sample was filtered 12 days after collection and the sample bottle was shaken before the aliquot was removed. Arsenic complexation to iron precipitates in the water was the reason for the decrease in $\mathrm{As}_{t o t}$ between collection and 12 days after collection (i.e. before ECAR treatment); thus the proper initial arsenic concentration to measure removal via complexation to iron precipitates is the original Astot measured in the sample as soon after collection as possible.

\subsection{Results and Discussion}

5.3.1 As $[\mathrm{III}]$ monitoring in samples of real groundwater

This section describes results and possible mechanisms of changing As[III] content of stored groundwater samples.

The ratio As[III]/Astot for TW 12 shows a clear decrease over 7 days, lowering 
from 0.96 to 0.052 (Table 5.2). The total arsenic decreases by only $15 \%$ in the same period (presumably due to complexation with settling iron precipitates in the water), and cannot account for the $95 \%$ decrease in As[III] after 7 days, or the $90 \%$ decrease after 4 days. As[III] is known to be slowly oxidized by dissolved $\mathrm{O}_{2}$, though the half-life reported in the literature is inconsistent. Eary \& Schramke (1990) measured an As[III] half-life of 1 - 3 years in equilibrium with atmospheric oxygen - much slower than the oxidation rate observed here. Kim \& Nriagu (2000) report a half-life of 4 - 9 days for As[III] in air-saturated groundwater with low iron content and $\mathrm{pH} 7.6-8.5$. This is still slower than the rate observed here, but is much closer. Two possible mechanisms for the observed reduction in As[III] are described in the following.

The first scenario involves a measurement error. As[III] will form complexes with natural iron precipitates in the water over time. ICPMS would detect this As[III] in the total arsenic measurement, but the large precipitates and associated As[III] would be filtered out in the As[III] speciation cartridge, underestimating the As[III] in the sample. For the $95 \%$ decrease seen in As[III] to be accounted for by complexation to iron precipitates, the removal capacity of the natural iron would have to be $0.551 \mathrm{mg} / \mathrm{L}$ As[III] for $0.1 \mathrm{mg} / \mathrm{L}$ iron (measured from TW 12 after 9 days storage via ICPMS - see Section 5.3.4), or equivalently, $55 \mathrm{mg}-\mathrm{As}[\mathrm{III}] / \mathrm{mg}-\mathrm{Fe}$. Measurements of As[III] removal during coprecipitation with iron after addition of $\mathrm{FeCl}_{3}$ have been reported as 0.002 $0.012 \mathrm{mg}-\mathrm{As}[\mathrm{III}] / \mathrm{mg}$ Fe at pH 7 (estimated from figure in Hering et al. (1996)). Thus it is unlikely that this effect could account for the entire observed rapid reduction in As[III]. 
The second scenario involves the oxidation of As[III] in conjunction with the oxidation of Fe[II] over time. Hug \& Leupin (2003) found that, parallel to the disappearance of $\mathrm{Fe}[\mathrm{II}]$ due to oxidation by dissolved $\mathrm{O}_{2}$, As[III] is partially oxidized to $\mathrm{As}[\mathrm{V}]$ on a time scale of hours. As[III] oxidation was attributed to reactive oxygen species that are formed as intermediates during the reduction of $\mathrm{O}_{2}$ with Fe[II]. The same group found that bicarbonate increases the fraction of oxidized As[III]. This could provide a possible pathway for the rapid As[III] oxidation seen.

The rapid oxidation of As[III] observed in TW 12 is very similar to the rapid oxidation observed in synthetic Bangladesh groundwater (Section 3.2.3). Figure 5.2 shows the percent As[III] (relative to initial concentration) remaining after 0 - 7 days for three standard synthetic Bangladesh water batches (SBGW-1 type) compared to TW 12 (data for SBGW taken from Section 3.2.3). The similarity in the behavior of As[III] oxidation between synthetic and real groundwater suggests that the same mechanism is responsible in both cases. However, synthetic Bangladesh groundwater batches contained no iron at all, requiring a different mechanism to be at play in each case or ruling out the Fe[II] oxidation theory above. Unfortunately, the uncertainty in the As[III]/Astot ratio measured (discussed in Section 5.2.3) makes it difficult to draw conclusions on the basis of this data alone. A repeated and more careful experiment is required for further speculation. 


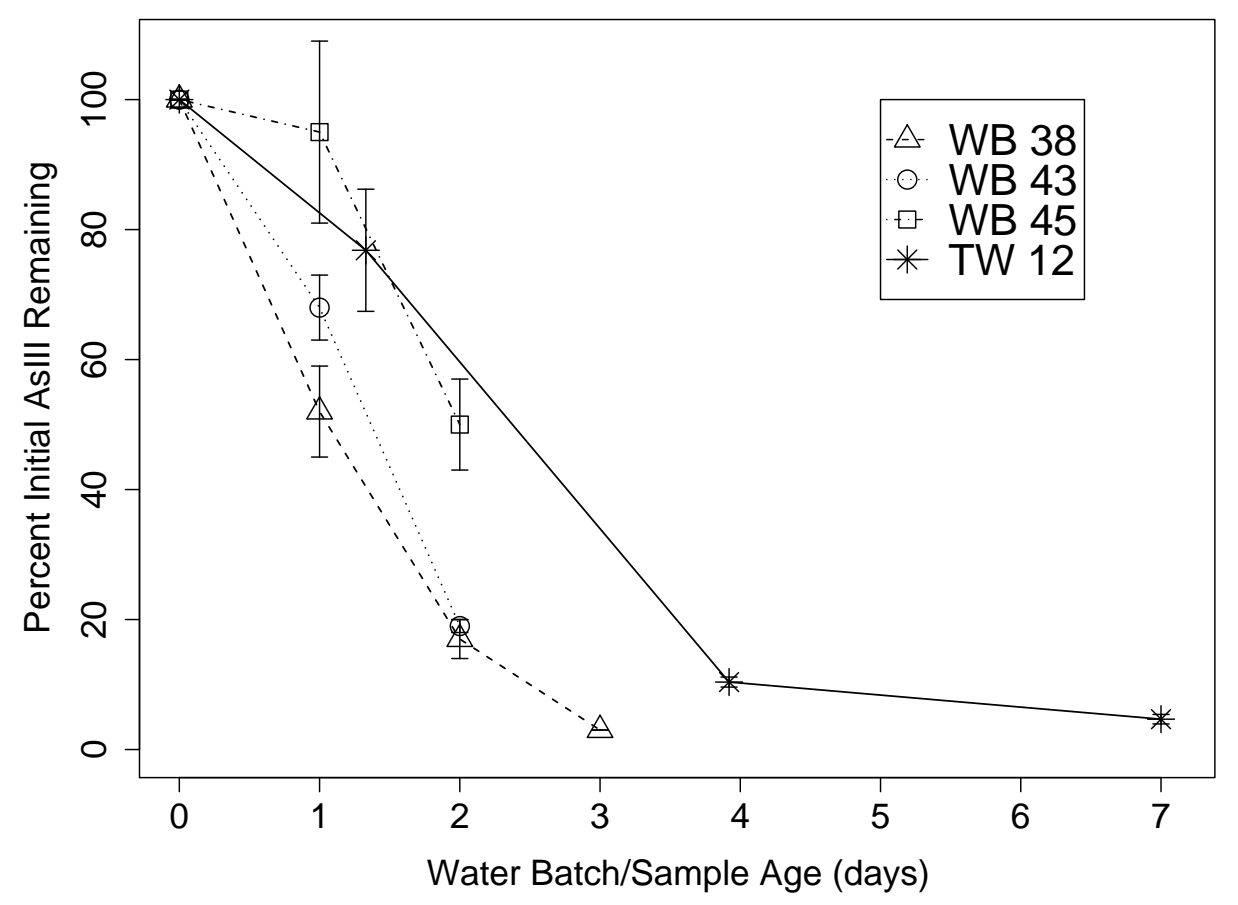

Figure 5.2: Percent As[III] relative to initial concentration remaining with time for three synthetic Bangladesh groundwater batches (WB; open symbols, dotted lines) and tube well water sample 12 (TW 12; solid symbol, solid line). The initial As[III] concentration for WB 38 and 45 was not measured, so the value is assumed to be the average As[III] concentration for all measured water batches, As[III $]=304 \pm 41 \mu \mathrm{g} / \mathrm{L}$. This uncertainty appears in all subsequent points, since the measured As[III] concentration must be divided by the initial As[III] concentration to calculate the percentage As[III] remaining. The uncertainty in As[III $] / \mathrm{As}[\mathrm{III}]_{\text {initial }}$ for TW 12 is estimated as the percentage decrease in $\mathrm{As}_{\text {tot }}$ relative to the initial concentration plus the ICPMS measurement uncertainty for both As[III $]$ and As $[\mathrm{III}]_{\text {initial }}$. 


\subsubsection{ECAR performance in real groundwater}

ECAR treatment was able to reduce the arsenic concentration in real Bangladesh groundwater to less than the WHO recommended limit $\left(\mathrm{As}_{t o t} \leq 10 \mu \mathrm{g} / \mathrm{L}\right)$ in every case (Figure 5.3), removing $97-99 \%$ of the total initial arsenic (Table 5.3). Pre-treatment arsenic concentrations ranged from $93-510 \mu \mathrm{g} / \mathrm{L}$, covering the concentrations found in $>96 \%$ of contaminated tube wells in Bangladesh (according the the BGS survey - Section 3.2.3). Coulombic dosage, residence time, and mixing time varied between tests (Table 5.3) to ensure removal to below the WHO limit. Dosages were purposely high to ensure complete removal; thus the dosages listed in Table 5.3 should not be mistaken for the minimum dosage required in real groundwater. Minimum dosage estimation will require further controlled testing.

From Figure 5.3, we see that final arsenic concentrations for tube well samples treated with ECAR were comparable to, and slightly smaller than, final arsenic concentrations for synthetic groundwater (SBGW-1). In fact, the arsenic concentration immediately after Coulombic dosing (designated by $t_{M}=0$ min in Figure 5.3 and Table 5.3) was already below the WHO limit for TW 4, 10, and 12 and quite close for TW 11, while for synthetic groundwater the concentration is still above the less stringent Bangladesh limit of $50 \mu \mathrm{g} / \mathrm{L}$. Due to the different ECAR conditions, it is difficult to make generalizations about the kinetics from this data alone, and further data and discussion is included in Section 5.3.3 below.

It is clear from Figure 5.3 and Table 5.3 that coprecipitation using the natural iron in the well water is insufficient on its own to reduce arsenic to below either the WHO 


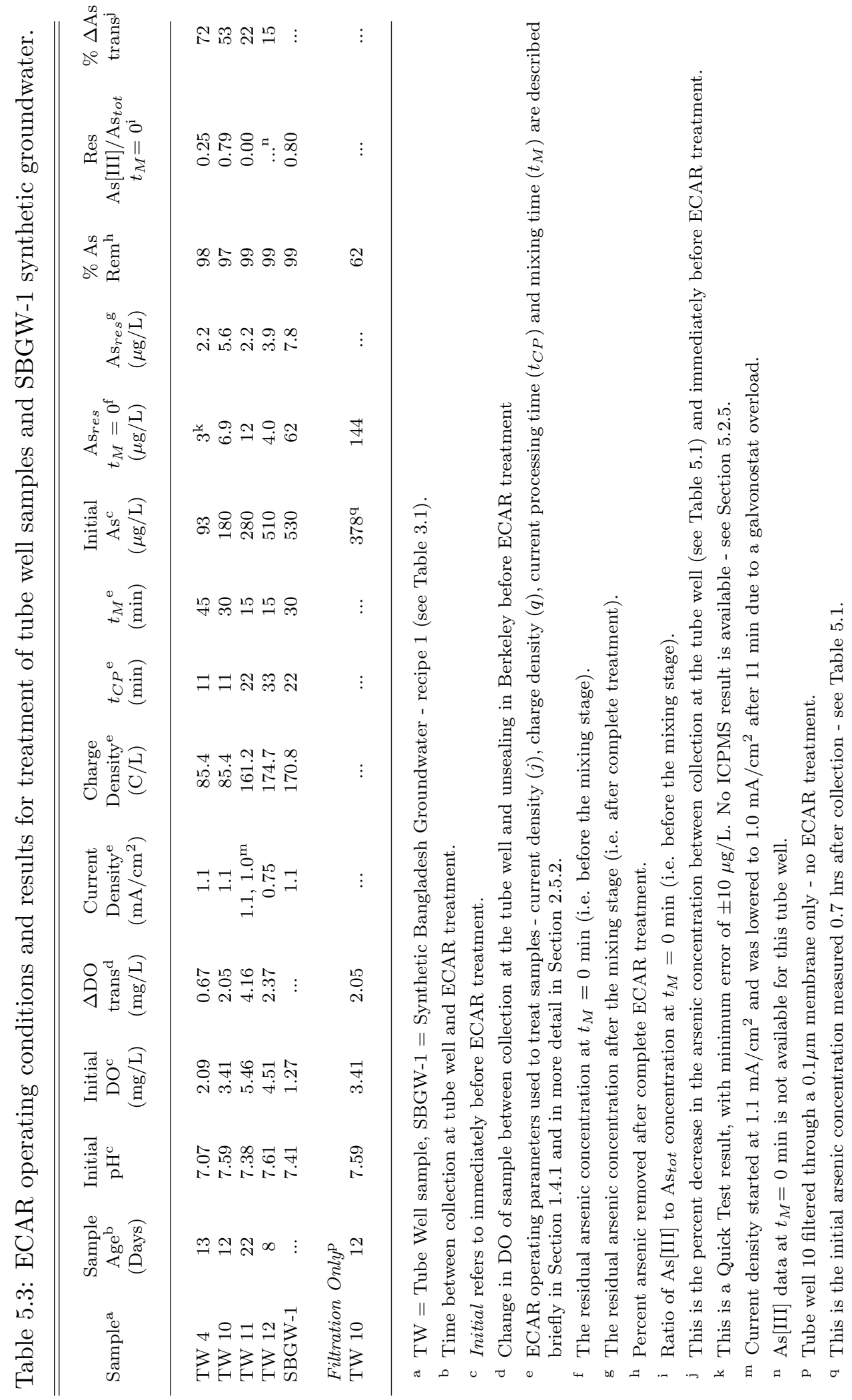




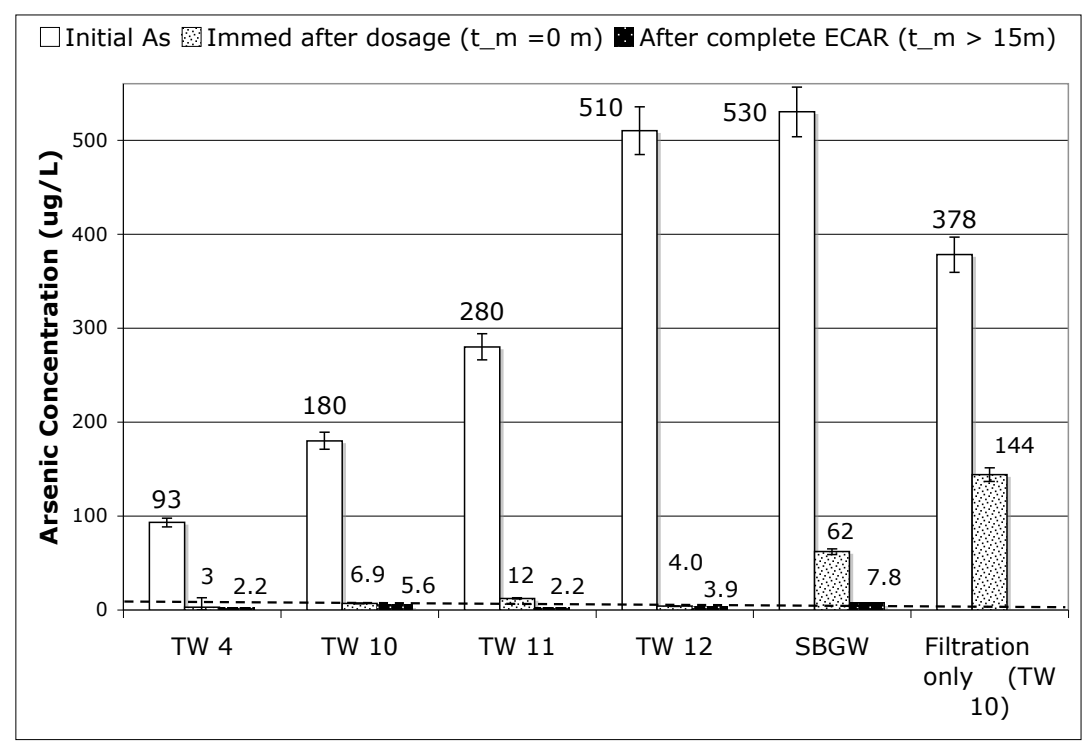

Figure 5.3: Arsenic concentrations before and after ECAR treatment for groundwater from four tube wells in Bangladesh along with SBGW-1 synthetic groundwater (SBGW), plus results from filtration alone (no ECAR) of TW 10 after 12 days of settling. Posttreatment concentrations are given for samples filtered immediately after Coulombic dosing (i.e. no mixing, of $t_{M}=0 \mathrm{~min}$ ) and after more than $15 \mathrm{~min}$ mixing $\left(t_{M}>15\right.$ min, see Table 5.3 for mixing times). Note that only Quick Test arsenic measurements are available for $\mathrm{TW} 4, t_{M}=0 \mathrm{~min}$; all other measurements are via ICPMS. The initial arsenic concentration for $T W 10$ and Filtered only $T W 10$ refers to the arsenic concentration immediately before ECAR treatment and $0.7 \mathrm{hrs}$ after collection from the tube well respectively. 
or Bangladesh limits in TW 10. Filtration alone reduced the total arsenic from $378 \mu \mathrm{g} / \mathrm{L}$ to $144 \mu \mathrm{g} / \mathrm{L}$, a reduction of only $62 \%$. It is difficult to compare this directly to ECAR, since ECAR treatment on the same tube well used the residual arsenic left in the water after some iron precipitation and settling occurred, resulting in a lower initial arsenic concentration $(180 \mu \mathrm{g} / \mathrm{L}$ compared to $378 \mu \mathrm{g} / \mathrm{L})$. However, TW 10 demonstrates that an initial settling period and filtration step before ECAR could lower the initial arsenic, allowing ECAR treatment with a lower Coulombic dosage.

The reduction in the arsenic concentration between collection at the tube well and immediately before ECAR treatment is most likely due to iron precipitation and subsequent adsorption in the well water (Section 5.2.4). Thus the magnitude of the decrease in arsenic can act as a relative measure of the amount of iron precipitation that occurred in each sample. Table 5.3 lists the percent decrease in arsenic during storage. As expected, TW 12, which appeared clear and free of precipitates, has the lowest decrease (15\% loss compared to $22-72 \%$ loss in other tube wells).

\subsubsection{Mixing time in real groundwater compared to synthetic ground- water}

It is clear from Figure 5.3 that residual arsenic concentrations immediately after Coulombic dosing $\left(t_{M}=0 \mathrm{~min}\right)$ are lower for tube well samples than for synthetic groundwater. In fact, residual arsenic for all tube well samples at $t_{M}=0$ min is below the WHO limit of $10 \mu \mathrm{g} / \mathrm{L}$. Significant time and money savings could result if the mixing stage were found to be unnecessary for removal to $<10 \mu \mathrm{g} / \mathrm{L}$ in Bangladesh groundwater. 
Further comparison of mixing time is allowed from TW 4 arsenic measurements at $t_{M}=$ 0, 15, 30, 45, and 60 min, shown in Figure 5.4. Corresponding measurements for synthetic groundwater at $t_{M}=0,15$, and $30 \mathrm{~min}$ are overlaid, showing a large discrepancy at $t_{M}=0 \mathrm{~min}$ and $15 \mathrm{~min}$, and convergence on the same value at $t_{M}=30 \mathrm{~min}$. The discrepancy is not due to the different residence time between TW 4 treatment and synthetic groundwater treatment because, from Table 5.3, the residence time is lower for TW 4 than for synthetic groundwater. Additional residence time for the synthetic groundwater would only reduce the arsenic concentration at $t_{M}=0 \mathrm{~min}$. However, it is likely that the discrepancy is due to the large difference in initial arsenic present in the synthetic groundwater compared to TW 4. Despite having twice the residence time, over four times as much arsenic must be removed from the synthetic water. This hypothesis would seem to be refuted by Figure 5.3, in which TW 12, with a comparable initial arsenic concentration to the synthetic groundwater $\left(A s_{\text {initial }}=511 \mu \mathrm{g} / \mathrm{L}\right.$ compared to $\left.A s_{\text {initial }}=533 \mu \mathrm{g} / \mathrm{L}\right)$ also has a significantly lower residual arsenic concentration at $t_{M}=0$ min. However, from Table 5.3, we see that the residence time for TW 12 is $50 \%$ longer than that of synthetic groundwater. This additional time could account for the discrepancy. TW 11 has the same residence time as the synthetic groundwater, and again shows a much lower residual arsenic at $t_{M}=0 \mathrm{~min}$. However the initial arsenic for TW 11 is lower compared to synthetic groundwater $\left(\mathrm{As}_{\mathrm{initial}}=278 \mu \mathrm{g} / \mathrm{L}\right.$ for $\left.\mathrm{TW} 11\right)$, which could account for the discrepancy. Thus it cannot be ruled out from these data that the required mixing time to reach $<10 \mu \mathrm{g} / \mathrm{L}$ for synthetic water is due to a higher initial arsenic concentration. 


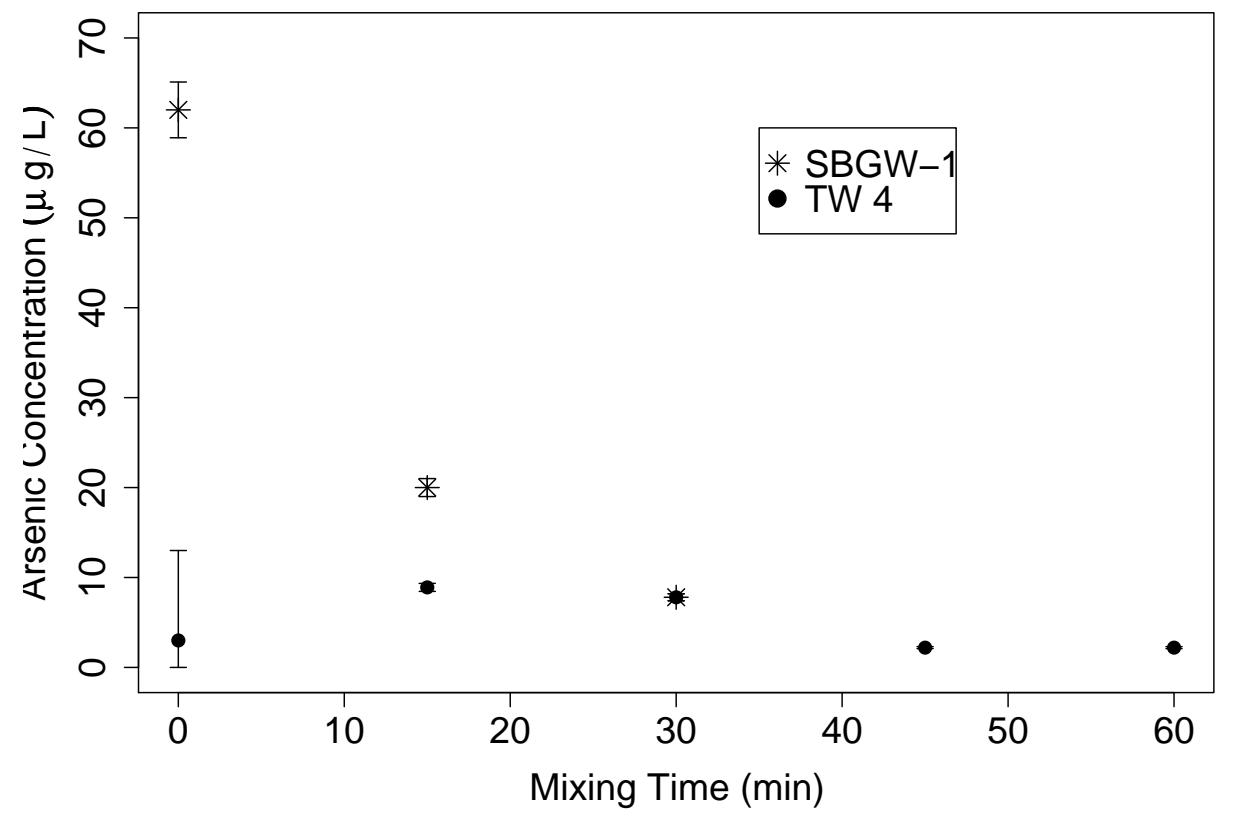

Figure 5.4: Residual arsenic concentration as function of mixing time $\left(t_{M}\right)$ for SBGW-1 synthetic groundwater $\left(\mathrm{As}_{\text {initial }}=530 \mu \mathrm{g} / \mathrm{L}\right)$ and real groundwater from Tube Well 4 $\left(\mathrm{TW} 4 ; \mathrm{As}_{\text {initial }}=93 \mu \mathrm{g} / \mathrm{L}\right)$. Note that points overlap at $t_{M}=30 \mathrm{~min}$. All arsenic concentrations were measured using ICPMS except for TW $4, t_{M}=0 \mathrm{~min}$, which was measured using the Quick Test (hence the higher error). Error in mixing time is +3 min for all points due to time of filtration. 
While the higher initial arsenic concentration cannot be ruled out, a second plausible explanation is the higher level of As[III] compared to As[V]. The synthetic groundwater batch started with a measured $\mathrm{As}[\mathrm{III}] / \mathrm{As}_{\text {tot }}$ ratio of 0.58 . The tube well samples are likely to have converted most of the original As[III] to As[V] by the time of ECAR testing, based on observations of rapid (within 7 days) oxidation discussed in Section 5.3.1. Initial As[III] aliquots were filtered through speciation cartidges, but the shaking of sample bottles prior to removing the aliquots likely increased the bias due to filtration of As[III] complexed to iron precipitates in the speciation cartridge (discussed in Section 5.2.3). The post-treatment As[III] concentration was measured after filtration through a $0.1 \mu \mathrm{m}$ membrane, and therefore should display little bias due to particulate filtration from the speciation cartridge (some bias will still occur due to precipitation that occurs after filtration through the $0.1 \mu \mathrm{m}$ membrane but before filtration through the speciation cartidge - artificially reducing the measured As[III $] / \mathrm{As}_{\text {tot }}$ ratio). The measured ratio at $t_{M}=0 \mathrm{~min}$ (in Table 5.3) for synthetic groundwater is 0.80 , indicating that most of the remaining arsenic is As[III]. The ratio for tube well samples varies from $0-0.79$, and in all cases As $[\mathrm{III}] \leq 4.4 \mu \mathrm{g} / \mathrm{L}$. This is consistent with both the hypothesis that very little As[III] was in the tube well samples to begin with and that high As[III] content is the cause of the increased mixing time needed in the synthetic groundwater. However, it is not enough to prove either theory.

These data suggest, but do not prove, that it might be possible to avoid or minimize the mixing stage in real Bangladesh water by letting the water sit for 4 days and allowing the As[III] to oxidize to As[V]. Further study is recommended. 


\subsubsection{Sample well comparison to BGS - co-occurring solutes}

One groundwater sample from each region visited (designated by Bangladesh Upazila) was analyzed for numerous co-occuring solutes after 11-19 days of storage to approximate the background composition of tube well samples immediately before ECAR treatment. Tube well samples collected from the same region were close together, suggesting similar geochemistry. The measured concentrations, shown in Table 5.4, can be compared to average concentrations found in the BGS (discussed more fully in Section 3.2.3) for shallow tube wells from the same Upazila (496 Upazilas cover the $150 \mathrm{~km}^{2}$ area of Bangladesh). These local statistics from BGS roughly approximate the background composition of the tube well before transport and storage, though the averages suffer from poor statistics (only a few wells exist in BGS from each region - 7 from Jhikargachha and 4 each from Abhaynagar and Sonargaon). Statistics derived from the full BGS database (covering 433 of the 496 Upazilas) as well as synthetic groundwater recipes are shown for comparison. All statistics derived from BGS include the average solute concentration for wells in the designated region with $\mathrm{As}_{t o t} \geq 10 \mu \mathrm{g} / \mathrm{L}$, along with the standard deviation (a rough estimate of the spread, since the distributions are not gaussian).

\section{Comparison of sampled tube wells to regional BGS concentrations}

Comparisons of measured tube well solute concentrations to BGS regional average concentrations can give a rough estimate of the effect of storage and transportation on tube well solute composition. Inter-well variation within a region is approximated by 


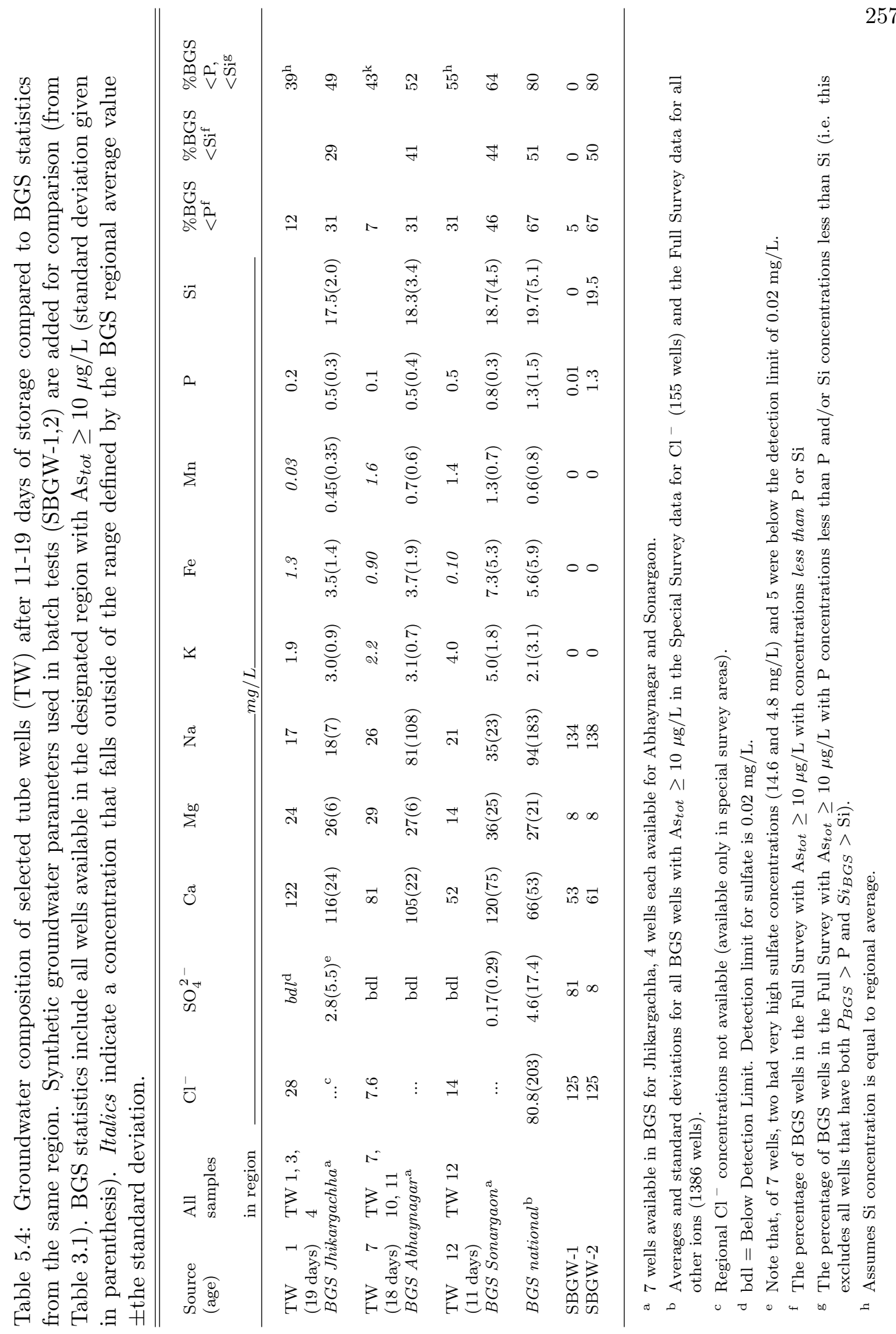


the standard deviation, which is admittedly uncertain due to a lack of gaussian distributions and low counting statistics.

Not surprisingly, Table 5.4 indicates that all aged TW samples tested contain below-average Fe concentrations for their region - this is likely an indicator of how much Fe has precipitated out of solution and/or stuck to the sides of the sample bottle. Oddly, TW 12, which contained no visible iron precipitate, has the lowest Fe concentration compared to the highest regional average values in BGS. This suggests that TW 12 contained a low Fe concentration to begin with. Mamtaz \& Bache (2001) reports that natural iron in Bangladesh groundwater of a concentration $F e[I I I]=66 * A s^{1.75}$ where $\mathrm{Fe}[\mathrm{III}]$, As are in $\mathrm{mg} / \mathrm{L}$ is sufficient to reduce arsenic concentrations to below $50 \mu \mathrm{g} / \mathrm{L}$ after shaking and 3 days settling. For TW 10 from Abhaynagar, initial arsenic at the tube well (2.7 hours after collection - Table 5.1) was $378 \mu \mathrm{g} / \mathrm{L}$, requiring $12 \mathrm{mg} / \mathrm{L}$ of iron(III) to reduce arsenic to $<50 \mu \mathrm{g} / \mathrm{L}$ using this method. Neither the measured value of iron in nearby TW 7 nor the regional average in Abhaynagar are large enough to support removal via natural arsenic alone, explaining the low $62 \%$ removal seen using settling and filtration alone (Figure 5.3) for TW 10.

Other concentrations in tube well samples that are low or high compared to regional averages include the Manganese (Mn) concentration for TW 1 and 7. Manganese is another metal that can form insoluble hydroxides upon exposure to oxygen, perhaps precipitating out with the iron. This could explain the lower than average concentration measured in TW 1. The presence of synthetic Mn oxyhydroxides was found to aid the rapid oxidation of As[III] (Devitre et al. (1991)) and could perhaps be one cause 
of extremely low As[III] concentrations found in TW 12 after 4 days (Section 5.3.1). However, the Mn level measured in TW 12 is right within the range expected for the region, suggesting little $\mathrm{Mn}$ has precipitated out and settled, though some could have been disturbed in transit. Synthetic groundwater contained no Mn to begin with, implying a different effect would have to be the cause of rapid oxidation in real groundwater compared to synthetic (which is possible). The Mn concentration measured for TW 7 is above both the regional and national averages, however, high Mn is not likely to affect adversely ECAR treatment.

Sulfate is lower than the regional average for TW 1. However, in this case, it does not imply that the sulfate concentration is abnormally low since 5 of the 7 wells used in the average contained no sulfate (i.e. levels were below detection limit of 0.02 mg/L). The remaining 2 wells contained abnormally high levels of sulfate, inflating the average.

TW 7 has low $\mathrm{K}$ concentrations compared to the regional average, but the concentration is right in the range of the national average. $\mathrm{Na}$ is also on the low side for this well, though it is within the regional range. Low Na and $\mathrm{K}$ could slightly decrease the conductivity of this water relative to average. This could help explain the low conductivity displayed by the overloading galvonostat during ECAR Coulombic dosing of TW 11 (Section 5.2.5). However, no overloading occurred during ECAR treatment of TW 4, likely to have even lower $\mathrm{Na}$ and $\mathrm{K}$ concentration based on the regional and measured TW 1 values (though higher Ca concentrations could make up for the low $\mathrm{Na}$ ). Phosphorous is within range, but on the low side for all aged tube well samples. 
This could be due to either a relatively low phosphorous concentration in the tube well at the time of collection, or adsorption of phosphate onto iron hydroxide precipitates in the sample bottle. In either case, phosphorous at the time of ECAR testing is representative of the phosphorous levels in the region.

While silicate was not measured in the aged tube well samples, the regional average concentrations of silica fall within a tight range for all available wells in the region. This suggests that the original silica concentration in our tube well samples was within this range as well, though little is known about how much may have been removed due to iron precipitation during aging.

All other co-occuring solutes are within the range defined by the average BGS regional value \pm the standard deviation.

\section{Comparison of sampled tube wells to national BGS concentrations}

A comparison of aged tube well solute concentrations and regional BGS averages

to the national BGS averages can help characterize how representative our tube well samples are to tube wells across Bangladesh, specifically in terms of interfering ions (see Section 2.4.6). The key solutes that can be detrimental to arsenic removal are phosphate, silicate, and bicarbonate and to some extent sulfate (see Section 2.4.6).

Bicarbonate representativeness cannot be characterized from the current data, both because it was not measured in our samples and because it is not available in the Full Survey data of the BGS. Phosphorous concentrations in both the aged tube well samples and BGS regional averages are 40-90\% lower than the national average. In the 
BGS Full Survey, only $31 \%$ of wells tested (with Astot $\geq 10 \mu \mathrm{g} / \mathrm{L}$ ) have phosphorous levels below $0.5 \mathrm{mg} / \mathrm{L}$, the highest value measured in the three aged samples. BGS regional average silica concentrations are $5-11 \%$ less than the national average, but well within one standard deviation. The lowest regional silica average, occurring in Jhikargachha, is greater than $29 \%$ of the BGS Full Survey wells (with $\mathrm{As}_{t o t} \geq 10 \mu \mathrm{g} / \mathrm{L}$ ), putting it firmly on the low side. Similar to the phosphorous concentration in the aged tube well samples, the initial silica is likely to have decreased further before ECAR treatment due to iron precipitation. Given these results, it is likely that the tube well samples used in ECAR treatment tests contained relatively low levels of key solutes such as phosphate and silicate compared to national averages.

Phosphate and silicate present together are known to have a more detrimental effect on arsenic removal than the combination of effects from either alone (Section 2.4.6). In Table 5.4, the percentage of BGS wells in the Full Survey with less P, less Si, or less $\mathrm{P}$ and $\mathrm{Si}$ combined than the measured values is listed (for aged TW samples, the Si concentration is assumed to be the regional average). Essentially, this estimates the percentage of BGS wells for which the combined detrimental effect of phosphate and silicate is likely to be worse than the effect seen in sample tube wells. By this metric, sample tube wells represent 39 - $55 \%$ of the tube wells in Bangladesh in terms of combined phosphate and silicate interference.

From Section 2.4.6, sulfate has been found to have little to no effect on As[V] adsorption to ferrihydrite. As[III] adsorption was found to decrease a few percent for $\mathrm{pH}$ $<7$ for $\mathrm{S}(\mathrm{VI}) /$ As ratios of 10:1 and 50:1. Assuming the national average concentration of 
sulfate $\left(4.6 \mathrm{mg} / \mathrm{L} \mathrm{SO}_{4}, 1.54 \mathrm{mg} / \mathrm{L} \mathrm{S}(\mathrm{VI})\right)$ and the initial arsenic concentrations of aged tube well samples used in ECAR experiments (Table 5.3), the ratio of $\mathrm{S}(\mathrm{VI}) /$ As ranges from 17:1 to 3:1, within the range of measured slight decrease in As[III] adsorption. The measured sulfate levels for aged tube well samples and BGS regional averages (Table 5.4) are therefore low with respect to possible detrimental effects due to sulfate that might occur in other regions. However, the effect on As[III] adsorption near $\mathrm{pH} 7$ is very small (1\%) even for 50:1 S(VI)/As, and not likely to effect ECAR treatment significantly. In fact, higher sulfate levels could aid ECAR efficiency due to the increase in water conductivity.

\subsection{Chapter Summary}

Real groundwater from a tube well in Bangladesh was collected from the Sonargaon Upazila (initial $\mathrm{As}_{\text {tot }}=600 \mu \mathrm{g} / \mathrm{L}$ ) and the $\mathrm{As}[\mathrm{III}] / \mathrm{As}_{\text {tot }}$ ratio was monitored up to 7 days after collection. A 95\% decrease in the initial As[III] concentration was observed over 7 days, with a $90 \%$ decrease after only 4 days. This is similar to the rapid oxidation seen in synthetic Bangladesh groundwater over a similar time scale. In real groundwater, $95 \%$ oxidation took slightly longer than in synthetic groundwater (7 days compared to 3 days). Observed oxidation is too rapid to be due to atmospheric oxygen alone. More careful measurements are required to determine the cause of rapid oxidation.

Real groundwater from Bangladesh was collected from high-arsenic (100 - 500 $\mu \mathrm{g} / \mathrm{L})$ tube wells in three regions and treated using ECAR. In every case, the final arsenic concentration was reduced to below the WHO recommended limit of $10 \mu \mathrm{g} / \mathrm{L}$. 
Simple filtration of one tube well sample (i.e. with no ECAR) was only able to reduce the arsenic concentration from $378 \mu \mathrm{g} / \mathrm{L}$ to $144 \mu \mathrm{g} / \mathrm{L}$ after 12 days of settling due to complexation with naturally occurring iron precipitates in the water, indicating that further treatment is necessary for this region. ECAR treatment in real groundwater was was able to reduce the arsenic concentration to $<12 \mu \mathrm{g} / \mathrm{L}$ in every case with no mixing time, compared to synthetic groundwater which required at least 30 minutes of mixing. This could be due to the low initial As[III]/Astot ratio of tested tube well water compared to synthetic groundwater, suggesting that oxidation of As[III] prior to ECAR treatment could remove the need for a mixing step. However, different residence times used for the ECAR treatment of tube well samples (to control dosage) make it difficult to generalize about the mixing time relative to synthetic groundwater; further experiments are recommended with residence time held constant.

Concentrations of several co-occuring solutes were measured in representative tube well water samples and compared to regional and national averages in the BGS. ECAR-tested tube wells were found to have low initial phosphate concentrations compared to regional and national tube wells (possibly due to complexation with iron precipitates and settling out of solution). Silica concentrations for BGS tube wells in each region were within range of national values. Assuming the BGS regional values for silica and all $\mathrm{P}$ and $\mathrm{Si}$ in the form of phosphate and silicate, measured tube wells contained more phosphate and silicate in combination than $39-55 \%$ of tube wells in Bangladesh. There was some suggestion through low sulfate, potassium, and sodium concentrations that conductivity might be lower than average in the tube wells tested. 
Valuable information was also gained on methods and procedures for sampling and testing arsenic-contaminated groundwater in Bangladesh. The importance of on-site ECAR testing was highlighted by issues of natural iron precipitation in the well water. Although the iron precipitation is likely to aid arsenic removal through adsorption of some arsenic, phosphate, and silicate, it adversely affects predictions of ECAR performance in groundwater straight from the tube well. In addition, valuable information was learned about the use and limitations of disposable speciation cartidges as a way to measure As[III] in water with particulate matter. 


\section{Chapter 6}

\section{Conclusions}

\subsection{Summary of results}

Electrochemical arsenic remediation (ECAR) using iron has been proposed as a potentially viable, low-cost, and effective technology for community scale arsenic remediation in rural Bangladesh. Two bench-top electrochemical cells have been designed and incorporated into a small-scale ECAR batch treatment procedure. Synthetic Bangladesh groundwater (SBGW) has been developed to mimic key environmental properties that could affect arsenic removal in Bangladesh, such as high levels of phosphate and silicate. Arsenic removal capacity - defined as the average amount of arsenic removed per coulomb between $600 \mu \mathrm{g} / \mathrm{L}$ and the WHO recommended maximum arsenic limit of $10 \mu \mathrm{g} / \mathrm{L}$ - has been proposed as a performance metric directly related to the operating costs of ECAR treatment. The arsenic removal capability to reach the WHO limit in the groundwater environment of Bangladesh and total treatment time have been proposed as additional 
key performance metrics for assessing ECAR.

The arsenic removal capability of ECAR has been demonstrated by reducing initial concentrations of $550-580 \mu \mathrm{g} / \mathrm{L}$ arsenic (including equal amounts of As[III] and $\mathrm{As}[\mathrm{V}])$ to below $10 \mu \mathrm{g} / \mathrm{L}$ in synthetic Bangladesh groundwater.

The effect of ECAR operating parameters - including current density, $j$, charge density, $q$, mixing time, $t_{M}$, and current processing time, $t_{C P}$ - on arsenic removal capacity and total treatment time has been explored, resulting in the following observations and measurements:

1. Increasing the duration of the post-electrolysis mixing stage in ECAR, controlled by $t_{M}$, can be used to increase arsenic removal capacity at the expense of increasing the treatment time.

2. Arsenic removal during the mixing stage is biphasic, with a period of rapid arsenic reduction between 10-40 minutes long (depending in part on $t_{C P}$ ) followed by slow reduction.

3. For a given value of $j, t_{M}$, and $t_{C P}$, the total charge density passed into solution determines the extent of arsenic removal, with increasing charge density leading to increased removal.

4. Arsenic removal capacity is approximately constant within certain ranges of current density, but highly variable between current density ranges. In order of decreasing removal capacity, the pattern discernible from batch tests is: $0.02 \mathrm{~mA} / \mathrm{cm}^{2}>$ $0.07 \mathrm{~mA} / \mathrm{cm}^{2}>0.30 \mathrm{~mA} / \mathrm{cm}^{2}-1.1 \mathrm{~mA} / \mathrm{cm}^{2}>5.0 \mathrm{~mA} / \mathrm{cm}^{2}-100 \mathrm{~mA} / \mathrm{cm}^{2}$. 
5. For a given value of $q$ and $j$, Arsenic removal capacity increases with increasing $t_{C P}$ at the expense of increasing the total treatment time.

6. No single set of operating parameters is optimal in terms of both arsenic removal capacity and treatment time.

7. The highest arsenic removal capacity for reduction of arsenic to the WHO limit in SBGW with a total treatment time of less than $2.5 \mathrm{hrs}$ was $3.7 \mu \mathrm{g}-\mathrm{As}_{\mathrm{rem}} / \mathrm{C}$, achieved at $j=1.1 \mathrm{~mA} / \mathrm{cm}^{2}, q=150 \mathrm{C} / \mathrm{L}, t_{C P}=69 \mathrm{~min}, t_{M}=60 \mathrm{~min}$.

8. The highest arsenic removal capacity overall for reduction of arsenic to the WHO limit in SBGW was $22 \mu \mathrm{g}-\mathrm{As}_{\mathrm{rem}} / \mathrm{C}$, achieved at $j=0.02 \mathrm{~mA} / \mathrm{cm}^{2}, q=25 \mathrm{C} / \mathrm{L}$, $t_{C P}=416 \min (\sim 7 \mathrm{hrs}), t_{M}=60 \mathrm{~min}$.

This assessment differs from previous assessments of operating parameter influence on arsenic removal via electrocoagulation (Kumar et al., 2004; Gomes et al., 2007) in the following key points: (1) current density was found to have an effect on arsenic removal capacity (though no effect was seen within the current density ranges studied by Kumar et al. (2004) and Gomes et al. (2007)), (2) $t_{C P}$ was found to have an effect on arsenic removal capacity that is independent of the total charge density and current density, and (3) the addition of a post-electrolysis mixing stage controlled by the parameter $t_{M}$ was added to allow for further increases in the arsenic removal capacity.

Post-synthesis ECAR-generated iron (hydr)oxides adsorbent, or PS-EGA adsorbent, was created by running the ECAR dosing process in synthetic Bangladesh groundwater containing no arsenic. Freshly made PS-EGA was able to reduce initial 
concentrations of $\sim 600 \mu \mathrm{g} / \mathrm{L}$ (including $\mathrm{As}[\mathrm{III}]$ and $\mathrm{As}[\mathrm{V}]$ in a $1: 1$ ratio) to $37 \mu \mathrm{g} / \mathrm{L}$ after 60 minutes of mixing and $22 \mu \mathrm{g} / \mathrm{L}$ after 48 hours of contact. ECAR treatment under comparable conditions was able to reduce arsenic to $10 \mu \mathrm{g} / \mathrm{L}$ after 60 minutes of mixing. The low final arsenic concentration using PS-EGA suggests that some of the initial $300 \mu \mathrm{g} / \mathrm{L}$ of As[III] was able to complex with PS-EGA directly, without electrochemical oxidation. Significantly however, the adsorbent was not able to reduce total arsenic levels to below the WHO limit $(10 \mu \mathrm{g} / \mathrm{L})$, indicating that the in-situ generation of EGA during ECAR is significant to its arsenic removal capability.

Arsenic removal capacity for PS-EGA (as $\mu \mathrm{g}-\mathrm{As}_{r e m} / \mathrm{mg}-\mathrm{Fe}^{3+}$ ) was $33 \%$ lower after 60 minutes of contact if the PS-EGA was allowed to age 60 minutes before coming into contact with arsenic. After 48 hours of contact, removal capacity was only $13 \%$ lower, however, the minimum arsenic concentration of achieved was $100 \mu \mathrm{g} / \mathrm{L}$. This indicates that removal capacity of EGA is highly sensitive to aging. This is likely due to the formation of aggregates. Once aggregates form, arsenic oxyanions must diffuse into them to find available sites. It is possible that the adsorption capacity of agedPS-EGA could catch up with that of fresh-PS-EGA given enough time for diffusion into aggregates to occur. However, given the constraints on treatment time proposed in Chapter 1, attempting to form PS-EGA at a central location and shipping the adsorbent to satellite treatment sites in place of on-site ECAR treatment is not advised.

The arsenic removal capacity of ECAR for As[III] appears to be approximately 3 times lower than the removal capacity for As[V]. This is consistent with both (1) a lower affinity of EGA for As[III] compared to As[V] or (2) slow oxidation of As[III] compared 
to the rate of $\mathrm{As}[\mathrm{V}]$ complexation, requiring more current processing time rather than more charge.

Freshly made PS-EGA (defined in previous paragraphs) was able to remove $90 \%$ of added As[III] after 60 minutes of contact in SBGW containing competitive ions such as phosphate, silicate, bicarbonate, and $\mathrm{As}[\mathrm{V}]$. No electrodes were present or external voltage applied during the contact time, preventing electrochemical oxidation of As[III]. This implies that EGA has some affinity for As[III] on the timescale of ECAR treatment, even in the presence of competing ions. However, ECAR is still capable of reducing As[III] concentrations lower than adsorption onto PS-EGA even after 48 hours of contact. These results suggest that (1) direct complexation of As[III] onto EGA may account for some portion of arsenic removal in ECAR and (2) some additional process (possibly electrochemical oxidation) is responsible for the reduction of As[III] down to the lowest achievable levels near the WHO limit.

Slow scan transient voltammetry was performed on the iron electrode at $\nu=$ $0.1 \mathrm{mV} / \mathrm{s}$ in $0.1 \mathrm{M} \mathrm{KClO}_{4}$ (plain perchlorate) solution and $0.1 \mathrm{M} \mathrm{KClO}_{4}$ plus $\mathrm{SBGW}$. A significant current drop in the SBGW scan relative to plain perchlorate solution suggests that species in the SBGW interfere with the dissolution processes on the iron electrode. This resulting increase in the overpotential required to reach a given current density in SBGW will add to the energy consumption of ECAR treatment. Since the overpotential is less severe at higher current densities, this could give high current densities some advantage over low current densities in terms of energy consumption. This could help offset disadvantages due to higher operating current. 
Electrode passivation, a common problem during electrocoagulation with aluminum electrodes, was not seen within the range of the polarization scan (up to current density $j=10 \mathrm{~mA} / \mathrm{cm}^{2}$ ). The lack of passivation in iron over the current density region that is most effective for arsenic removal is promising in terms of electrode longevity, maintenance requirements, and the ability to operate in monopolar mode.

The polarization scan revealed that the electrode potential over the entire range of scanned current density (i.e. $j \leq 10 \mathrm{~mA} / \mathrm{cm}^{2}$ ) is well below the thermodynamic equilibrium potential for oxygen evolution. This rules out oxygen evolution as a cause of the decrease in arsenic removal capacity seen at higher current density.

Initial chemical characterization (including only Fe and As) of arsenic-laden EGA showed that the waste from a representative ECAR treatment of synthetic Bangladesh groundwater $\left(j=1.1 \mathrm{~mA} / \mathrm{cm}^{2}, q=175 \mathrm{C} / \mathrm{L}\right.$, initial arsenic $=590 \mu \mathrm{g} / \mathrm{L}$, final arsenic $=5.0 \mu \mathrm{g} / \mathrm{L}, \mathrm{SBGW}-2$ recipe) contained $3300 \mathrm{ppm}$ arsenic by mass with an As $/$ Fe mass ratio of 0.0076 . Measured waste quantity was $0.33 \mathrm{mg}$ waste per liter of treated water. Estimates of the expected amount of waste per treated liter based on the amount of arsenic removed from solution at $q=150 \mathrm{C} / \mathrm{L}$ were a factor of 3 higher than the measured amount of waste produced.

SEM images showed that EGA is very homogenous and made up of rounded popcorn-like particles with a primary aggregate size of 50-100 nm. Arsenic breakthrough experiments with increasing pore size showed that the cluster size of EGA is as least $800 \mathrm{~nm}$. XRF mapping of the EGA sludge confirmed homogeneity and showed that arsenic is evenly captured across EGA. 
A direct of comparison of $0.1 \mu$ mfiltration to sedimentation (i.e. quiescent settling followed by decantation) as a means to separate EGA from potable water revealed that approximately three days of settling is required to achieve comparable arsenic removal results. The long required settling time is likely due to the small particle size of EGA. An alternative low-cost method of EGA separation compatible with the small particle size will have to be explored.

Fe XANES spectra for EGA generated at $j=0.02-100 \mathrm{~mA} / \mathrm{cm}^{2}$ were consistent with an iron oxidation state of $3+$ at all current densities. This disproved the hypothesis that EGA generated at $j=0.02 \mathrm{~mA} / \mathrm{cm}^{2}$ is dominated by Fe[II]-based (hydr)oxides, as suggested by polarization curves in Section 3.3.10.

Comparisons between Fe EXAFS spectra of EGA sludge generated at current densities $j=0.02,1.1,5.0$, and $100 \mathrm{~mA} / \mathrm{cm}^{2}$ revealed no discernible difference in iron (hydr)oxide structure with current density. Comparisons between arsenic EXAFS spectra similarly revealed no discernible difference in the structure of the iron (hydr)oxide bound to arsenic with current density. These results are inconsistent with the hypothesis that differences in EGA composition with current density account for the differences in arsenic removal capacity with current density measured in Chapter 3. An alternative hypothesis was formulated that attributes the increase in arsenic removal capacity to an increase in arsenic capture by the electrode surface layer at low current density. This could potentially be accounted for by the increase in the electrode-surface-area to active-solution-volume ratio $(\mathrm{A} / \mathrm{V})$ used in batch tests to accommodate the factor of 5000 difference in current density between $j=0.02$ and $100 \mathrm{~mA} / \mathrm{cm}^{2}$. Further experiments 
are planned to test this hypothesis.

Based on Fe EXAFS spectral comparisons between EGA sludge and reference iron (hydr)oxide compounds, EGA sludge was found to be consistent with a Fe[III] oxyhydroxide similar in structure to 2-line ferrihydrite. This is consistent with the Fe XANES results showing a $3+$ oxidation state for iron.

The arsenic bonding structure responsible for arsenic removal in ECAR treatment was found to be very consistent with arsenate adsorbed to a Fe[III] oxyhydroxide. Based on key differences in line shape, the spectrum is inconsistent with scorodite. Further analysis is underway to determine the bonding structure from characteristic bond lengths determined by shell-by-shell fits to the EXAFS spectra.

Combining these results, two important conclusions are reached - (1) there is no significant difference in the arsenic removal mechanism of ECAR during operation at different current densities (at least in the bulk solution), and (2) based on EXAFS spectral comparisons, the arsenic removal mechanism in ECAR is consistent with arsenate adsorption onto a homogenous Fe[III] oxyhydroxide similar in structure to 2-line ferrihydrite. Further analysis of the EXAFS spectra beyond the scope of this thesis will allow for more concrete conclusions.

Real groundwater from a tube well in Bangladesh was collected from the Sonargaon Upazila (initial $\mathrm{As}_{t o t}=600 \mu \mathrm{g} / \mathrm{L}$ ) and the $\mathrm{As}[\mathrm{III}] / \mathrm{As}_{t o t}$ ratio was monitored up to 7 days after collection. A $95 \%$ decrease in the initial As[III] concentration was observed over 7 days, with a $90 \%$ decrease after only 4 days. This is similar to the rapid oxidation seen in synthetic Bangladesh groundwater over a similar time scale. In real groundwater, 
$95 \%$ oxidation took slightly longer than in synthetic groundwater (7 days compared to 3 days). Observed oxidation is too rapid to be due to atmospheric oxygen alone.

Real groundwater from Bangladesh was collected from high-arsenic (100 - 500 $\mu \mathrm{g} / \mathrm{L})$ tube wells in three regions and treated using ECAR. In every case, the final arsenic concentration was reduced to below the WHO recommended limit of $10 \mu \mathrm{g} / \mathrm{L}$. Simple filtration of one tube well sample (i.e. with no ECAR) was only able to reduce the arsenic concentration from $378 \mu \mathrm{g} / \mathrm{L}$ to $144 \mu \mathrm{g} / \mathrm{L}$ after 12 days of settling due to complexation with naturally occurring iron precipitates in the water, indicating that further treatment is necessary for this region. ECAR treatment in real groundwater was was able to reduce the arsenic concentration to $<12 \mu \mathrm{g} / \mathrm{L}$ in every case with no mixing time, compared to synthetic groundwater which required at least 30 minutes of mixing.

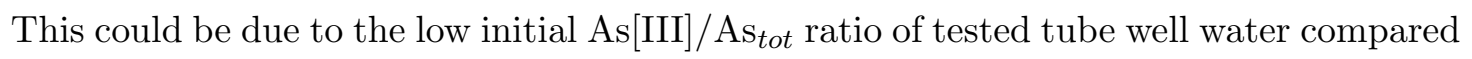
to synthetic groundwater, suggesting that oxidation of As[III] prior to ECAR treatment could remove the need for a mixing step. However, different current processing times used for the ECAR treatment of tube well samples (to control dosage) make it difficult to generalize about the mixing time relative to synthetic groundwater; further experiments are recommended with current processing time held constant.

Concentrations of several co-occuring solutes were measured in representative tube well water samples and compared to regional and national averages in the BGS. ECAR-tested tube wells were found to have low initial phosphate concentrations compared to regional and national tube wells (possibly due to complexation with iron precipitates and settling out of solution). Silica concentrations for BGS tube wells in each 
region were within range of national values. Assuming the BGS regional values for silica and all $\mathrm{P}$ and $\mathrm{Si}$ in the form of phosphate and silicate, measured tube wells contained more phosphate and silicate in combination than 39-55\% of tube wells in Bangladesh. There was some suggestion through low sulfate, potassium, and sodium concentrations that conductivity might be lower than average in the tube wells tested.

Valuable information was also gained on methods and procedures for sampling and testing arsenic-contaminated groundwater in Bangladesh. The importance of on-site ECAR testing was highlighted by issues of natural iron precipitation in the well water. Although the iron precipitation is likely to aid arsenic removal through adsorption of some arsenic, phosphate, and silicate, it adversely affects predictions of ECAR performance in groundwater straight from the tube well. In addition, valuable information was learned about the use and limitations of disposable speciation cartidges as a way to measure As[III] in water with particulate matter.

\subsection{Future Work}

A prototype continuous flow device based on ECAR was design and fabricated by a team of graduate students (under the direction of Ashok Gadgil and myself) in Spring 2008. The prototype was found to successfully reduce $600 \mu \mathrm{g} / \mathrm{L}$ As to below the WHO limit in synthetic Bangladesh groundwater (data to be published at a later date). The prototype was recently operated in Bangladesh and arsenic-contaminated region of Cambodia, achieving very promising results. It is currently being prepared for more rigorous field-testing in Bangladesh. 


\section{Bibliography}

Abbgy, A., Kelly, T., Lawrie, C., \& Riggs, K. (2002). Quick Arsenic Test kit. Tech. rep., Battelle.

Abdullah, M., Shiyu, Z., \& Mosgren, K. (1995). Arsenic and selenium species in the oxic and anoxic waters of the Oslof-jord, Norway. March Pollution Bulletin, 31, 116-126.

Abuzaid, N. S., Bukhari, A. A., \& Al-Hamouz, Z. M. (2002). Ground water coagulation using soluble stainless steel electrodes. Advances in Environmental Research, 6(3), $325-333$.

Aggett, J., \& Kriegman, M. (1987). Preservation of arsenic (III) and arsenic (V) in samples of sediment interstitial water. The Analyst, 112, 153-157.

Ahmad, J., Goldar, B., Misra, S., \& Jakaruya, M. (2003). Willingness to pay for arsenicfree, safe drinking water in Bangladesh. Tech. rep., The World Bank.

Ahmed Basha, C., Bhadrinarayana, N. S., Anantharaman, N., \& Meera Sheriffa Begum, K. M. (2008). Heavy metal removal from copper smelting effluent using electrochemical cylindrical flow reactor. Journal of Hazardous Materials, 152(1), 71-78.

Ahsan, H., Chen, Y., Parvez, F., Argos, M., Hussain, A. I., Momotaj, H., Levy, D., van Geen, A., Howe, G., \& Graziano, J. (2006). Health effects of arsenic longitudinal study (HEALS): Description of a multidisciplinary epidemiologic investigation. Journal of Exposure Science and Environmental Epidemiology, 16, 191-205.

Alam, M. G. M., Tokunaga, S., \& Maekawa, T. (2001). Extraction of arsenic in a synthetic arsenic-contaminated soil using phosphate. Chemosphere, 43(8), 1035-1041.

Anderson, M. A., Ferguson, J. F., \& Gavis, J. (1976). Arsenate adsorption on amorphous aluminum hydroxide. Journal of Colloid and Interface Science, 54 (3), 391-399.

Anderson, M. A., \& Malotky, D. (1979). The adsorption of protolyzable anions on hydrous oxides at the isoelectric pH. Journal of Colloid and Interface Science, 72, $413-427$.

Anderson, M. A., Tejedor-Tejedor, M. I., \& Stanforth, R. R. (1985). Influence of aggregation on the uptake kinetics of phosphate by goethite. Environmental Science $\mathbb{6}$ Technology, 19(7), 632-637. 
Anderson, P. R., \& Benjamin, M. M. (1985). Effect of silicon on the crystallization and adsorption properties of ferric oxides. Environmental Science $\& 5$ Technology, 19(11), $1048-1053$.

Anderson, R., Thompson, M., \& Culbard, E. (1986). Selective reduction of arsenic species by continuous hydride generation. The Analyst, 111, 1153-1158.

Appelo, C., Van Der Weiden, M., Tournassat, C., \& Charlet, L. (2002). Surface complexation of ferrous iron and carbonate on ferrihydrite and the mobilization of arsenic. Environmental Science \&5 Technology, 36(14), 3096-3103.

Arienzo, M., Adamo, P., Chiarenzelli, J., Bianco, M. R., \& De Martino, A. (2002). Retention of arsenic on hydrous ferric oxides generated by electrochemical peroxidation. Chemosphere, 48(10), 1009-1018.

Balasubramanian, N., \& Madhavan, K. (2001). Arsenic removal from industrial effluent through electrocoagulation. Chemical Engineering \& Technology, 24(5), 519-521.

Bang, S., Johnson, M. D., Korfiatis, G. P., \& Meng, X. (2005a). Chemical reactions between arsenic and zero-valent iron in water. Water Research, 39(5), 763-770.

Bang, S., Korfiatis, G. P., \& Meng, X. G. (2005b). Removal of arsenic from water by zero-valent iron. Journal of Hazardous Materials, 121 (1-3), 61-67.

Bard, A., \& Faulkner, L. (2001). Electrochemical Methods: Fundamentals and Applications. Wiley and Sons, Inc.

Beck, E. C., Giannini, A. P., \& Ramirez, E. R. (1974). Electrocoagulation clarifies food wastewater. Food Technology, 28(2), 18.

Beck, R. (2007). World facing arsenic timebomb. BBC News: http://news.bbc.co.uk/2/hi/science/nature/6968574.stm.

Belongia, B. M., Haworth, P. D., Baygents, J. C., \& Raghavan, S. (1999). Treatment of alumina and silica chemical mechanical polishing waste by electrodecantation and electrocoagulation. Journal of the Electrochemical Society, 146(11), 4124-4130.

Benjamin, M. M. (1983). Adsorption and surface precipitation of metals on amorphous iron oxyhydroxide. Environmental Science \&5 Technology, 17(11), 686-692.

Benjamin, M. M. (2002). Water Chemistry. McGraw-Hill.

BGS (2001). Arsenic contamination of groundwater in Bangladesh. Tech. Rep. WC/00/19, British Geological Survey.

Bhattacharya, P., Jacks, G., Ahmed, K. M., Routh, J., \& Khan, A. A. (2002). Arsenic in groundwater of the Bengal Delta Plain aquifers in Bangladesh. Bulletin of Environmental Contamination and Toxicology, 69(4), 538-545. 
Birch, W., Pring, A., Reller, A., \& Schmalle, H. (1993). Bernalite, Fe(OH)3, a new mineral from Broken Hill, New South Wales: Description and structure. American Mineralogist, 78, 827-834.

Bissen, M., \& Frimmel, F. (2003). Arsenic - a review. part II: Oxidation of arsenic and its removal in water treatment. Acta Hydrochimica Et Hydrobiologica, 31 (2), 97-107.

Biswas, N., \& Lazarescu, G. (1991). Removal of oil from emulsions using electrocoagulation. International Journal of Environmental Studies, 38, 65.

Bond, A. (2002). Broadening Electrochemical Horizons. New York: Oxford University Press.

Borho, M., \& Wilderer, P. (1996). Optimized removal of arsenate(III) by adaptation of oxidation and precipitation processes to the filtration step. Water Science and Technology, 34(9), 25-31.

Borho, M., \& Wilderer, P. (1997). A reliable method for preservation and detennination of 138 arsenate(III) concentrations in groundwater and water works samples. AquaJournal of Water Supply: Research and Technology, 46, 138-143.

Boyle, D., Turner, R., \& Hall, G. (1998). Anomalous arsenic concentrations in groundwaters of an island community, Bowen Island, British Columbia. Environmental Geochemistry and Health, 20(4), 1573-2983.

BRAC (2000). Combating a deadly menace: Early experience with a community-based arsenic mitigation project in Bangladesh. Tech. rep., BRAC.

Burgess, W., \& Ahmed, K. (2006). Arsenic in aquifers of the Bengal Basin. In R. Naidu, E. Smith, G. Owens, P. Bhattacharya, \& P. Nadebaum (Eds.) Managing Arsenic in the Environment, (pp. 31-58). Australia: CSIRO.

Chang, C.-C., Ho, S.-C., Tsai, S.-S., \& Yang, C.-Y. (2004). Ischemic heart disease mortality reduction in an arseniasis-endemic area in southwestern Taiwan after a switch in the tap-water supply system. Journal of Toxicology $\& 5$ Environmental Health: Part A, 67(17), 1353-1361.

Charlet, L., \& Manceau, A. (1993). Structure, formation, and reactivity of hydrous oxide particles: insights from x-ray absorption spectroscopy. In J. Buffle, \& H. van Leeuwen (Eds.) Environmental Particles, vol. 2 of Environmental Analytical and Physical Chemistry series. Ann Arbor: Lewis Publishers.

Chen, G. (2004). Electrochemical technologies in wastewater treatment. Separation and Purification Technology, 38(1), 11-41.

Chen, X. M., Chen, G. H., \& Yue, P. L. (2000). Separation of pollutants from restaurant wastewater by electrocoagulation. Separation and Purification Technology, 19(1-2), $65-76$. 
Cheng, R. C., Wang, H. C., \& Beuhler, M. D. (1994). Enhanced coagulation for arsenic removal. Journal of the American Water Works Association, 86(9), 79-90.

Cherry, J. A., Shaikh, A. U., Tallman, D. E., \& Nicholson, R. V. (1979). Arsenic species as an indicator of redox conditions in groundwater. Journal of Hydrology, 43(1-4), 373-392.

Chiang, L. C., Chang, J. E., \& Tseng, S. C. (1997). Electrochemical oxidation pretreatment of refractory organic pollutants. Water Science and Technology, 36(2-3), $123-130$.

Chiu, H.-F., Ho, S.-C., \& Yang, C.-Y. (2004). Lung cancer mortality reduction after installation of tap-water supply system in an arseniasis-endemic area in Southwestern Taiwan. Lung Cancer, 46(3), 265-270.

Chowdhury, U. K., Biswas, B. K., Chowdhury, T. R., Samanta, G., Mandal, B. K., Basu, G. C., Chanda, C. R., Lodh, D., Saha, K. C., \& Mukherjee, S. K. (2000). Groundwater arsenic contamination in Bangladesh and West Bengal, India. Environmental Health Perspectives, 108(5), 393-397.

Collier, W. (1912). Description of plants at Oklahoma City, Okla and Santa Monica, Calif. Engng Rec., 66, 55.

Cornell, R., \& Schwertmann, U. (2000). The Iron Oxides. Wiley-VCH.

Crisp, P., \& Chowdhury, A. (2001). Design of a low-cost purification system for the removal of arsenic from tubewell water in Bangladesh and India.

Daneshvar, N., Khataee, A. R., Ghadim, A. R. A., \& Rasoulifard, M. H. (2007). Decolorization of $\mathrm{Ci}$ acid yellow 23 solution by electrocoagulation process: Investigation of operational parameters and evaluation of specific electrical energy consumption (SEEC). Journal of Hazardous Materials, 148(3), 566-572.

Davies, J. (1995). The hydrogeochemistry of alluvial aquifers in central Bangladesh. In H. Nash, \& G. McCall (Eds.) Groundwater Quality, (pp. 9-18). New York: Chapman \& Hall.

Davis, C. C., Knocke, W. R., \& Edwards, M. (2001). Implications of aqueous silica sorption to iron hydroxide: Mobilization of iron colloids and interference with sorption of arsenate and humic substances. Environmental Science $\& 5$ Technology, 35 (15), 31583162 .

Davis, J. A., Fuller, C. C., \& Cook, A. D. (1987). A model for trace metal sorption processes at the calcite surface: Adsorption of $\mathrm{Cd} 2+$ and subsequent solid solution formation. Geochimica Et Cosmochimica Acta, 51(6), 1477-1490.

Davis, J. A., \& Kent, D. B. (1990). Surface complexation modeling in aqueous geochemistry. In M. Hichella, \& A. White (Eds.) Mineral-Water Interface Geochemistry, (pp. 177-248). Washington D.C.: Mineralogical Society of America. 
Deng, Y. (1997). Formation of iron(III) hydroxides from homogeneous solutions. Water Research, $31(6), 1347-1354$.

Devitre, R., Belzile, N., \& Tessier, A. (1991). Speciation and adsorption of arsenic on diagenetic iron oxyhydroxides. Limnology and Oceanography, 36 (7), 1480-1485.

Dixit, S., \& Hering, J. G. (2003). Comparison of arsenic(V) and arsenic(III) sorption onto iron oxide minerals: Implications for arsenic mobility. Environmental Science $\mathcal{G}$ Technology, 37(18), 4182-4189.

Do, J. S., \& Chen, M. L. (1994). Decolourization of dye-containing solutions by electrocoagulation. Journal of Applied Electrochemistry, 24(8), 785-790.

Driehaus, W., Jekel, M., \& Hildebrandt, U. (1998). Granular ferric hydroxide - a new adsorbent for the removal of arsenic from natural water. Journal of Water Services Research and Technology-Aqua, 47(1), 30-35.

Dzombak, D., \& Morel, F. (1990). Surface Complexation Modelling-Hydrous Ferric Oxide. New York: John Wiley.

Eary, L., \& Schramke, J. (1990). Rates of inorganic oxidation reactions involving dissolved oxygen. In Melchior, \& Bassett (Eds.) Chemical Modeling of Aqueous Systems II. Washington, D.C.: American Chemical Society.

Edwards, M. (1994). Chemistry of arsenic removal during coagulation and Fe-Mn oxidation. Journal of the American Water Works Association, 86(9), 64-78.

Endyuskin, P. N., Selezenkin, S. V., \& Dyumaev, K. M. (1983). Electrochemical purification of wastewaters from production of organic-dyes. Journal of Applied Chemistry of the Ussr, 56(5), 1100-1102.

EPA, U. (1998). Method 6020A: Inductive coupled plasma-mass spectrometry. Tech. rep., U.S. EPA.

Farquhar, M. L., Charnock, J. M., Livens, F. R., \& Vaughan, D. J. (2002). Mechanisms of arsenic uptake from aqueous solution by interaction with goethite, lepidocrocite, mackinawite, and pyrite: An X-ray absorption spectroscopy study. Environmental Science $\&$ Technology, 36(8), 1757-1762.

Farrell, J., Wang, J. P., O’Day, P., \& Conklin, M. (2001). Electrochemical and spectroscopic study of arsenate removal from water using zero-valent iran media. Environmental Science \& Technology, 35(10), 2026-2032.

Fendorf, S., Eick, M. J., Grossl, P., \& Sparks, D. L. (1997). Arsenate and chromate retention mechanisms on goethite .1. surface structure. Environmental Science 8 Technology, 31(2), 315-320. 
Ferguson, J. F., \& Anderson, M. A. (1974). Chemical form of arsenic in water supplies and their removal. In A. Rubin (Ed.) Chemistry of Water Supply, Treatment, and Distribution, (pp. 137-158). Ann Arbor, MI: Ann Arbor Science.

Ferguson, J. F., \& Gavis, J. (1972). Review of arsenic cycle in natural waters. Water Research, 6(11), 1259-1274.

Ford, R. G. (2002). Rates of hydrous ferric oxide crystallization and the influence on coprecipitated arsenate. Environmental Science \& Technology, 36(11), 2459-2463.

Foster, A. (1999). Partitioning and Transformation of Arsenic and Selenium in Natural and Laboratory Systems. Ph.D. thesis, Stanford University.

Foster, A. L. (2003). Spectroscopic investigations of arsenic species in solid phases. In A. H. Welch, \& K. Stollenwerk (Eds.) Arsenic in Groundwater. Boston: Kluwer Academic Press.

Fuller, C. C., Davis, J. A., \& Waychunas, G. A. (1993). Surface-chemistry of ferrihydrite .2. kinetics of arsenate adsorption and coprecipitation. Geochimica Et Cosmochimica Acta, 57(10), 2271-2282.

Gallagher, P. A., Schwegel, C. A., Parks, A., Gamble, B. M., Wymer, L., \& Creed, J. T. (2004). Preservation of $\mathrm{As}(\mathrm{III})$ and $\mathrm{As}(\mathrm{V})$ in drinking water supply samples from across the United States using EDTA and acetic acid as a means of minimizing iron-arsenic coprecipitation. Environmental Science \& Technology, 38(10), 2919-2927.

Gao, Y., \& Mucci, A. (2001). Acid base reactions, phosphate and arsenate complexation, and their competitive adsorption at the surface of goethite in $0.7 \mathrm{M} \mathrm{NaCl}$ solution. Geochimica Et Cosmochimica Acta, 65(14), 2361-2378.

Ghosh, M., \& Teoh, R. (1985). Adsorption of arsenic on hydrous aluminum oxide. In Toxic and Hazardous Wastes: Proceedings of the Seventh Mid-Atlantic Industrial Waste Conference June 23-25, 1985., (pp. 139-155). Lancaster, PA.: Technomic Publishing Co.

Goldberg, S. (1986). Chemical modeling of arsenate adsorption on aluminum and ironoxide minerals. Soil Science Society of America Journal, 50(5), 1154-1157.

Goldberg, S., \& Johnston, C. T. (2001). Mechanisms of arsenic adsorption on amorphous oxides evaluated using macroscopic measurements, vibrational spectroscopy, and surface complexation modeling. Journal of Colloid and Interface Science, 234(1), $204-216$.

Gomes, J. A. G., Daida, P., Kesmez, M., Weir, M., Moreno, H., Parga, J. R., Irwin, G., McWhinney, H., Grady, T., Peterson, E., \& Cocke, D. L. (2007). Arsenic removal by electrocoagulation using combined Al-Fe electrode system and characterization of products. Journal of Hazardous Materials, 139(2), 220-231. 
Grossl, P. R., Eick, M., Sparks, D. L., Goldberg, S., \& Ainsworth, C. C. (1997). Arsenate and chromate retention mechanisms on goethite .2. kinetic evaluation using a pressurejump relaxation technique. Environmental Science \& Technology, 31(2), 321-326.

Grossl, P. R., \& Sparks, D. L. (1995). Evaluation of contaminant ion adsorption/desorption on goethite using pressure jump relaxation kinetics. Geoderma, 67(12), $87-101$.

Gulledge, J. H., \& Oconnor, J. T. (1973). Removal of arsenic (V) from water by adsorption on aluminum and ferric hydroxides. Journal of the American Water Works Association, 65(8), 548-552.

Gupta, S. K., \& Chen, K. Y. (1978). Arsenic removal by adsorption. Journal Water Pollution Control Federation, 50(3), 493-506.

Gurses, A., Yalcin, M., \& Dogar, C. (2002). Electrocoagulation of some reactive dyes: a statistical investigation of some electrochemical variables. Waste Management, 22(5), 491-499.

Hansen, H. K., Nunez, P., \& Grandon, R. (2006). Electrocoagulation as a remediation tool for wastewaters containing arsenic. Minerals Engineering, 19(5), 521-524.

Hansen, H. K., Nunez, P., Raboy, D., Schippacasse, I., \& Grandon, R. (2007). Electrocoagulation in wastewater containing arsenic: Comparing different process designs. Electrochimica Acta, 52(10), 3464-3470.

Harvey, C. F., Swartz, C. H., Badruzzaman, A. B. M., Keon-Blute, N., Yu, W., Ali, M. A., Jay, J., Beckie, R., Niedan, V., Brabander, D., Oates, P. M., Ashfaque, K. N., Islam, S., Hemond, H. F., \& Ahmed, M. F. (2002). Arsenic mobility and groundwater extraction in Bangladesh. Science, 298(5598), 1602-1606.

Hem, J. (1961). Stability field diagrams as aids in iron chemistry studies. Journal of the American Water Works Association, 53(2), 211-232.

Hering, J. G., Chen, P. Y., Wilkie, J. A., Elimelech, M., \& Liang, S. (1996). Arsenic removal by ferric chloride. Journal of the American Water Works Association, 88(4), $155-167$.

Hiemstra, T., \& Van Riemsdijk, W. H. (1999). Surface structural ion adsorption modeling of competitive binding of oxyanions by metal (hydr)oxides. Journal of Colloid and Interface Science, 210(1), 182-193.

Hingston, F. J., Posner, A. M., \& Quirk, J. P. (1971). Competitive adsorption of negatively charged ligands on oxide surfaces. Discussions of the Faraday Society, 52, $334-342$.

Holm, T. (2002). Effects of co32-/bicarbonate, si, and po43- on arsenic sorption to HFO. Journal of the American Water Works Association, 94(4), 174-181. 
Holm, T., Anderson, M. A., Iverson, D., \& Stanforth, R. (1979). Heterogeneous of arsenic in aquatic systems. American Chemical Society Symposium Series, 93, 711-736.

Holt, P. K., Barton, G. W., Wark, M., \& Mitchell, C. A. (2002). A quantitative comparison between chemical dosing and electrocoagulation. Colloids and Surfaces APhysicochemical and Engineering Aspects, 211 (2-3), 233-248.

Honeyman, B. D., \& Santschi, P. H. (1991). Coupling adsorption and particle aggregation: Laboratory studies of colloidal pumping' using 59-Fe-labeled hematite. Environmental Science \& Technology, 25, Pages: 1739-1747.

Hopenhayn-Rich, C., Biggs, M., Kalman, D., Moore, L., \& Smith, A. (1996). Arsenic methylation patterns before and after changing from high to lower concentrations of arsenic in drinking water. Environmental Health Perspectives, 104(11), 1200-1207.

Hsia, T. H., Lo, S. L., \& Lin, C. F. (1992). As(V) adsorption on amorphous iron-oxide - triple layer modeling. Chemosphere, 25(12), 1825-1837.

Hsia, T. H., Lo, S. L., Lin, C. F., \& Lee, D. (1994). Characterization of arsenate adsorption on hydrous iron oxide using chemical and physical methods. Characterization of arsenate adsorption on hydrous iron oxide using chemical and physical methods, $85(11), 1-7$.

Hug, S. J., \& Leupin, O. (2003). Iron-catalyzed oxidation of arsenic(III) by oxygen and by hydrogen peroxide; ph-dependent formation of oxidants in the Fenton reaction. Environmental Science 83 Technology, 37(12), 2734-2742.

Ibanez, J. G., Singh, M. M., \& Szafran, Z. (1998). Laboratory experiments on electrochemical remediation of the environment. Part 4: Color removal of simulated wastewater by electrocoagulation-electroflotation. Journal of Chemical Education, 75(8), $1040-1041$.

Islam, F. S., Boothman, C., Gault, A. G., Polya, D. A., \& Lloyd, J. R. (2005). Potential role of the $\mathrm{Fe}(\mathrm{III})$-reducing bacteria geobacter and geothrix in controlling arsenic solubility in Bengal delta sediments. Mineralogical Magazine, 69(5), 865-875.

Islam, F. S., Gault, A. G., Boothman, C., Polya, D. A., Charnock, J. M., Chatterjee, D., \& Lloyd, J. R. (2004). Role of metal-reducing bacteria in arsenic release from Bengal delta sediments. Nature, 430(6995), 68-71.

Jackson, B. P., \& Miller, W. P. (2000). Effectiveness of phosphate and hydroxide for desorption of arsenic and selenium species from iron oxides. Soil Science Society of America Journal, 64 (5), 1616-1622.

Jain, A., \& Loeppert, R. H. (2000). Effect of competing anions on the adsorption of arsenate and arsenite by ferrihydrite. Journal of Environmental Quality, 29(5), 14221430 . 
Jain, A., Raven, K. P., \& Loeppert, R. H. (1999). Arsenite and arsenate adsorption on ferrihydrite: Surface charge reduction and net $\mathrm{OH}$ - release stoichiometry. Environmental Science \& Technology, 33(8), 1179-1184.

Jovanovic, V., \& Hackerman, N. (1998). Electrochemical passivation of iron in NO3-, SO42-, and ClO4- solutions. Journal of Physical Chemistry B, 102(49), 9855-9860.

Kahn, M. (2007). Arsenic in water a risk to 140 million people. Reuters: http://www.alertnet.org/thenews/newsdesk/L29757483.htm.

Kaufmann, R., Sorensen, B., Rahman, M., Streatfield, K., \& Persson, L. (2001). Addressing the public health crisis caused by arsenic contamination of drinking water in Bangladesh. Tech. rep., South Asia Health, Nutrition, and population Unit, World Bank.

Kim, M.-J., \& Nriagu, J. (2000). Oxidation of arsenite in groundwater using ozone and oxygen. The Science of The Total Environment, 247(1), 71-79.

Kim, M. J., Nriagu, J., \& Haack, S. (2000). Carbonate ions and arsenic dissolution by groundwater. Environmental Science $\&$ Technology, 34(15), 3094-3100.

Kolitsch, U. (1998). Bernalite from Clara Mine, Germany, and the incorporation of tungsten in minerals containing ferric iron. Canad. Min., 36, 1211-1216.

Koparal, A. S., \& Ogutveren, U. B. (2002). Removal of nitrate from water by electroreduction and electrocoagulation. Journal of Hazardous Materials, 89(1), 83-94.

Korte, N. E., \& Fernando, Q. (1991). A review of arsenic(iii) in groundwater. Critical Reviews in Environmental Control, 21(1), 1-39.

Kumar, P. R., Chaudhari, S., Khilar, K. C., \& Mahajan, S. P. (2004). Removal of arsenic from water by electrocoagulation. Chemosphere, 55(9), 1245-1252.

Kurbatov, M. H., \& Wood, G. B. (1952). Rate of adsorption of cobalt ions on hydrous ferric oxide. The Journal of Physical Chemistry, 56(6), 698-701.

Kyle, M., \& Christiani, D. (2008). Environmental arsenic exposure and diabetes. Journal of the Americal Medical Association, 300(7), 845-846.

Lackovic, J. A., Nikolaidis, N. P., \& Dobbs, G. M. (2000). Inorganic arsenic removal by zero-valent iron. Environmental Engineering Science, 17(1), 29-39.

Leckie, J. O., Benjamin, M. M., Hayes, K., Kaufman, G., \& Altmann, S. (1980). Adsorption/coprecipitation of trace elements from water with iron oxyhydroxide. Tech. rep., Stanford Univ., CA (USA). Dept. of Civil Engineering.

Liu, F., De Cristofaro, A., \& Violante, A. (2001). Effect of pH, phosphate and oxalate on the adsorption/desorption of arsenate on/from goethite. Soil Science, 166(3), 197208. 
Lowers, H. A., Breit, G. N., Foster, A. L., Whitney, J., Yount, J., Uddin, M. N., \& Muneem, A. A. (2007). Arsenic incorporation into authigenic pyrite, Bengal Basin sediment, Bangladesh. Geochimica Et Cosmochimica Acta, 71(11), 2699-2717.

Lytle, D., \& Snoeyink, V. (2002). Effect of ortho- and polyphosphates on the properties of iron particles and suspensions. Journal of the American Water Works Association, $94(10), 87-99$.

Madajewicz, M., Pfaff, A., van Geen, A., Graziano, J., Hussein, I., Momotaj, H., Sylvi, R., \& Ahsan, H. (2008). Can information alone change behavior? Response to arsenic contamination of groundwater in Bangladesh (vol 84, pg 731, 2007). Journal of Development Economics, 85(1-2), 349-349.

Mameri, N., Yeddou, A. R., Lounici, H., Belhocine, D., Grib, H., \& Bariou, B. (1998). Defluoridation of septentrional Sahara water of north Africa by electrocoagulation process using bipolar aluminium electrodes. Water Research, 32(5), 1604-1612.

Mamtaz, R., \& Bache, D. (2001). Reduction of arsenic in groundwater by coprecipitation with iron. Journal of Water Supply: Research 83 Technology- AQUA, 50, 313-324.

Manceau, A. (1995). The mechanism of anion adsorption on iron-oxides - evidence for the bonding of arsenate tetrahedra on free $\mathrm{Fe}(\mathrm{O}, \mathrm{OH})(6)$ edges. Geochimica Et Cosmochimica Acta, 59(17), 3647-3653.

Manceau, A., \& Combes, J. M. (1988). Structure of Mn and Fe oxides and oxyhydroxides - a topological approach by EXAFS. Physics and Chemistry of Minerals, 15(3), 283295.

Manning, B. A., Fendorf, S. E., \& Goldberg, S. (1998). Surface structures and stability of arsenic(iii) on goethite: Spectroscopic evidence for inner-sphere complexes. Environmental Science \&5 Technology, 32(16), 2383-2388.

Manning, B. A., \& Goldberg, S. (1996a). Modeling arsenate competitive adsorption on kaolinite, montmorillonite and illite. Clays and Clay Minerals, 44(5), 609-623.

Manning, B. A., \& Goldberg, S. (1996b). Modeling competitive adsorption of arsenate with phosphate and molybdate on oxide minerals. Soil Science Society of America Journal, 60(1), 121-131.

Marcus, M., MacDowell, A., R., C., Manceau, A., Miller, T., Padmore, H., \& Sublett, R. (2004). Beamline 10.3.2 at ALS: a hard x-ray microprobe for environmental and materials sciences. Journal of Synchrotron Radiation, 11, 239-247.

Martin, T. A., \& Kempton, J. H. (2000). In situ stabilization of metal-contaminated groundwater by Hydrous Ferric Oxide: An experimental and modeling investigation. Environmental Science \& Technology, 34(15), 3229-3234. 
Mathieu, J., Gadgil, A., Kowolik, K., \& Addy, S. (2008). Removing arsenic from contaminated drinking water in rural Bangladesh: recent fieldwork results and policy implications. In Safe Drinking Water: Where Science Meets Policy. Chapel Hill, NC.

Matteson, M. J., Dobson, R. L., Glenn, R. W., Kukunoor, N. S., Waits, W. H., \& Clayfield, E. J. (1995). Electrocoagulation and separation of aqueous suspensions of ultrafine particles. Colloids and Surfaces A-Physicochemical and Engineering Aspects, $104(1), 101-109$.

Mayer, T. D., \& Jarrell, W. M. (2000). Phosphorus sorption during iron(II) oxidation in the presence of dissolved silica. Water Research, 34 (16), 3949-3956.

Melitas, N., Conklin, M., \& Farrell, J. (2002a). Electrochemical study of arsenate and water reduction on iron media used for arsenic removal from potable water. Environmental Science \& Technology, 36(14), 3188-3193.

Melitas, N., Wang, J. P., Conklin, M., O’Day, P., \& Farrell, J. (2002b). Understanding soluble arsenate removal kinetics by zerovalent iron media. Environmental Science 86 Technology, 36(9), 2074-2081.

Meng, X., Bang, S., \& Korfiatis, G. P. (2000). Effects of silicate, sulfate, and carbonate on arsenic removal by ferric chloride. Water Research, 34 (4), 1255-1261.

Meng, X., Korfiatis, G. P., Jing, C., \& Christodoulatos, C. (2001). Redox transformations of arsenic and iron in water treatment sludge during aging and TCLP extraction. Environmental Science \& Technology, 35(17), 3476-3481.

Meng, X., \& Wang, W. (1998). Speciation of arsenic by disposable cartridges. In Book of posters of the third International Conference on arsenic exposure and health effects: Society of Environmental Geochemistry and Health. Denver: University of Colorado Denver.

Meng, X. G., Korfiatis, G. P., Bang, S. B., \& Bang, K. W. (2002). Combined effects of anions on arsenic removal by iron hydroxides. Toxicology Letters, 133(1), 103-111.

Misawa, T. (1973). Thermodynamic consideration for Fe-H2O system at 25 degrees C. Corrosion Science, 13(9), 659-676.

Mishra, D. (2006). Electrochemical Deactivation of Nitrate, Arsenate, and Trichloroethylene. Ph.D. thesis, University of Arizona.

Mishra, D., \& Farrell, J. (2005). Evaluation of mixed valent iron oxides as reactive adsorbents for arsenic removal. Environmental Science $\&$ Technology, 39(24), 96899694.

Mollah, M. Y. A., Morkovsky, P., Gomes, J. A. G., Kesmez, M., Parga, J., \& Cocke, D. L. (2004). Fundamentals, present and future perspectives of electrocoagulation. Journal of Hazardous Materials, 114(1-3), 199-210. 
Moore, L. E., Smith, A. H., Hopenhayn-Rich, C., Biggs, M. L., Kalman, D. A., \& Smith, M. T. (1997). Decrease in bladder cell micronucleus prevalence after intervention to lower the concentration of arsenic in drinking water. Cancer Epidemiology Biomarkers \& Prevention, 6(12), 1051-1056.

Navas-Acien, A., Silbergeld, E., Pastor-Barriuso, R., \& Guallar, E. (2008). Arsenic exposure and prevalence of type 2 diabetes in US adults. Journal of the American Medical Association, 300(7), 814-822.

Ng, K., Ujang, Z., \& Le-Clech, P. (2004). Arsenic removal technologies for drinking water treatment. Reviews in Environmental Science and Bio/Technology, 3, 43-53.

Nickson, R. T., McArthur, J. M., Ravenscroft, P., Burgess, W. G., \& Ahmed, K. M. (2000). Mechanism of arsenic release to groundwater, Bangladesh and West Bengal. Applied Geochemistry, 15(4), 403-413.

O’Day, P. A., Rehr, J. J., Zabinsky, S. I., \& Brown, J., G. E. (1994). Extended X-ray Absorption Fine Structure (EXAFS) analysis of disorder and multiple-scattering in complex crystalline solids. Journal of the American Chemical Society, 116(7), 29382949 .

O'Melia, C. (1987). Particle-particle interactions. In W. Stumm (Ed.) Aquatic Surface Chemistry, (pp. 385-403). J. Wiley.

O'Reilly, S. E., Strawn, D. G., \& Sparks, D. L. (2001). Residence time effects on arsenate adsorption/desorption mechanisms on goethite. Soil Science Society of America Journal, 65(1), 67-77.

Paktunc, D., Foster, A., \& Laflamme, G. (2003). Speciation and characterization of arsenic in Ketza River mine tailings using X-ray absorption spectroscopy. Environmental Science $\&$ Technology, 37(10), 2067-2074.

Panizza, M., Bocca, C., \& Cerisola, G. (2000). Electrochemical treatment of wastewater containing polyaromatic organic pollutants. Water Research, 34(9), 2601-2605.

Parga, J. R., Cocke, D. L., Valenzuela, J. L., Gomes, J. A., Kesmez, M., Irwin, G., Moreno, H., \& Weir, M. (2005a). Arsenic removal via electrocoagulation from heavy metal contaminated groundwater in La Comarca Lagunera Mexico. Journal of Hazardous Materials, 124(1-3), 247-254.

Parga, J. R., Cocke, D. L., Valverde, V., Gomes, J. A. G., Kesmez, M., Moreno, H., Weir, M., \& Mencer, D. (2005b). Characterization of electrocoagulation for removal of chromium and arsenic. Chemical Engineering \& Technology, 28(5), 605-612.

Persin, F., \& Rumeau, M. (1989). Electrochemical treatment of waters and effluents. Tribune de l'eau, 42(3), 45-66. 
Petrusevski, B., Boere, J., Shahidullah, S. M., Sharma, S. K., \& Schippers, J. C. (2002). Adsorbent-based point-of-use system for arsenic removal in rural areas. Aqua- Journal of Water Supply: Research and Technology, 51(3), 135-144.

Petrusevski, B., Sharma, S., Schippers, J. C., \& Shordt, K. (2007). Arsenic in drinking water. Tech. rep., IRC International Water and Sanitation Centre.

Phutdhawong, W., Chowwanapoonpohn, S., \& Buddhasukh, D. (2000). Electrocoagulation and subsequent recovery of phenolic compounds. Analytical Sciences, 16(10), $1083-1084$.

Pierce, M. L., \& Moore, C. B. (1982). Adsorption of arsenite and arsenate on amorphous iron hydroxide. Water Research, 16(7), 1247-1253.

Pinisakul, A., Polprasert, C., Parkpian, P., \& Satayavivad, J. (2002). Arsenic removal efficiency and mechanisms by electro-chemical precipitation process. Water Science and Technology, 46(9), 247-254.

Pogrebnaya, V. L., Klimenko, A. A., Bokovikova, T. N., Tsymbal, E. P., \& Pronina, N. P. (1995). Purification of waste water of heavy metals by electrocoagulation. Chemical and Petroleum Engineering, 31(5), 280-281.

Posselt, H. S., Anderson, F. J., \& Weber, W. J. (1968). Cation sorption on colloidal hydrous manganese dioxide. Environmental Science \& Technology, 2(12), 1087-1093.

Pouet, M. F., \& Grasmick, A. (1995). Urban waste-water treatment by electrocoagulation and flotation. Water Science and Technology, 31(3-4), 275-283.

Rahman, M. M., Sengupta, M. K., Ahamed, S., Chowdhury, U. K., Hossain, M., Das, B., Lodh, D., Saha, K. C., Pati, S., \& Kaies, I. (2005). The magnitude of arsenic contamination in groundwater and its health effects to the inhabitants of the Jalangi; one of the 85 arsenic affected blocks in West Bengal, India. Science of the Total Environment, 338(3), 189-200.

Raven, K. P., Jain, A., \& Loeppert, R. H. (1998). Arsenite and arsenate adsorption on ferrihydrite: Kinetics, equilibrium, and adsorption envelopes. Environmental Science \& Technology, 32(3), 344-349.

Ravenscroft, P. (2003). An overview of the hydrogeology of Bangladesh. In A. Rahman, \& P. Ravenscroft (Eds.) Groundwater Resources and Development in Bangladesh. Dhaka: Bangladesh Center for Advanced Studies: University Press.

Rehr, J. J., Deleon, J. M., Zabinsky, S. I., \& Albers, R. C. (1991). Theoretical xray absorption fine-structure standards. Journal of the American Chemical Society, $113(14), 5135-5140$.

Roberts, L. C., Hug, S. J., Ruettimann, T., Billah, M. M., Khan, A. W., \& Rahman, M. T. (2004). Arsenic removal with iron(II) and iron(III) in waters with high silicate and phosphate concentrations. Environmental Science \& Technology, 38(1), 307-315. 
Roman-Ross, G., Cuello, G. J., Turrillas, X., Fernandez-Martinez, A., \& Charlet, L. (2006). Arsenite sorption and co-precipitation with calcite. Chemical Geology, 233(34), 328-336.

Roy, J. (2008). Economic benefits of arsenic removal from ground water - a case study from West Bengal, India. Science of the Total Environment, 397(1-3), 1-12.

Rushing, J. C., McNeill, L. S., \& Edwards, M. (2003). Some effects of aqueous silica on the corrosion of iron. Water Research, 37(5), 1080-1090.

Ryden, J. C., Syers, J. K., \& Tillman, R. W. (1987). Inorganic anion sorption and interactions with phosphate sorption by hydrous ferric oxide gel. European Journal of Soil Science, 38(2), 211-217.

Saha, J. C., Dikshit, A. K., Bandyopadhyay, M., \& Saha, K. C. (1999). A review of arsenic poisoning and its effects on human health. Critical Reviews in Environmental Science and Technology, 29(3), 281-313.

Saha, K. (1995). Chronic arsenical dermatoses from tube-well water in West Bengal during 1983-87. Indian Journal of Dermatology, 40, 1-12.

Sato, M., Kimura, M., Yamashita, M., Konishi, H., Fujimoto, S., Tabira, Y., Doi, T., Nagoshi, M., Suzuki, S., Kamimura, T., Nakayama, T., \& Ohtsuka, T. (2005). Atomicstructure characterization of passive film of fe by grazing incidence x-ray scattering at SPring-8. In P. Marcus, \& V. Maurice (Eds.) Passivation of metals and Semiconductors, and Properties of Thin Oxide Layers - 9th International Symposium. Paris, France: Elsevier.

Schenk, J., \& Weber, W. (1968). Chemical interactions of dissolved silica with iron(II) and iron(III). Journal of the American Water Works Association, 60, 199-212.

Shen, Y. S. (1973). Study of arsenic removal from drinking-water. Journal of the American Water Works Association, 65(8), 543-548.

Sigg, L., \& Stumm, W. (1981). The interaction of anions and weak acids with the hydrous goethite ([alpha]-FeOOH) surface. Colloids and Surfaces, 2(2), 101-117.

Smedley, P. L., \& Kinniburgh, D. G. (2002). A review of the source, behaviour and distribution of arsenic in natural waters. Applied Geochemistry, 17(5), 517-568.

Smith, A. H., Lingas, E. O., \& Rahman, M. (2000). Contamination of drinking-water by arsenic in Bangladesh: a public health emergency. Bulletin of the World Health Organization, 78(9).

Sorg, T. J., \& Logsdon, G. S. (1978). Treatment technology to meet the interim primary drinking water regulations for inorganics: Part 2. Journal of the American Water Works Association, 70(7), 379-393. 
Sposito, G. A. (1986). On distiguishing adsorption from surface precipitation. In J. Davies, \& K. Hayes (Eds.) Geochemical Processes at Mineral Surfaces, (pp. 217228). American Chemical Society Symposium Series.

Stollenwerk, K. (2003). Geochemical processes controlling transport of arsenic in groundwater: A review of adsorption. In A. H. Welch, \& K. Stollenwerk (Eds.) Arsenic in Groundwater. Boston: Kluwer Academic Publishers.

Stollenwerk, K., \& Kipp, K. (1990). Simulation of molybdate transport with different rate-controlled mechanisms. In D. Melchior, \& R. Bassett (Eds.) Chemical Modeling of Aqueous Systems II: Washington, (pp. 243-257). American Chemical Society Symposium Series.

Stumm, W., \& Morgan, J. (1996). Aquatic Chemistry. John Wiley \& Sons, Inc.

Su, C. M., \& Puls, R. W. (2001). Arsenate and arsenite removal by zerovalent iron: Kinetics, redox transformation, and implications for in situ groundwater remediation. Environmental Science \& Technology, 35(7), 1487-1492.

Sun, X. H., \& Doner, H. E. (1996). An investigation of arsenate and arsenite bonding structures on goethite by FTIR. Soil Science, 161(12), 865-872.

Swedlund, P. J., \& Webster, J. G. (1999). Adsorption and polymerisation of silicic acid on ferrihydrite, and its effect on arsenic adsorption. Water Research, 33(16), $3413-3422$.

Szynkarczuk, J., Kan, J., Hassan, T. A. T., \& Donini, J. C. (1994). Electrochemical coagulation of clay suspensions. Clays and Clay Minerals, 42(6), 667-673.

Tang, Y. K., \& Furtak, T. E. (1991). Study of underpotential deposition of metals using the quartz crystal microbalance. Electrochimica Acta, 36(11-12), 1869-1872.

Teo, B. K., \& Joy, D. (1981). EXAFS Spectroscopy, Techniques and Applications. New York, NY: Plenum Press.

Toner, B. M., Santelli, C. M., Marcus, M. A., Wirth, R., Chan, C. S., McCollom, T. M., Bach, W., \& Edwards, K. J. (2008). Biogenic iron oxide formation at Mid-Ocean Ridge hydrothermal vents: Juan de Fuca Ridge. Geochimica et Cosmochimica Acta (accepted).

Tsai, C. T., Lin, S. T., Shue, Y. C., \& Su, P. L. (1997). Electrolysis of soluble organic matter in leachate from landfills. Water Research, 31(12), 3073-3081.

UNICEF (1999). Progotir Pathey (on the road to progress); acheiving the goals for children in Bangladesh. Tech. rep., UNICEF.

UNICEF (2006). Arsenic mitigation in Bangladesh fact sheet. Tech. rep., UNICEF. 
van Green, A., Robertson, A. P., \& Leckie, J. O. (1994). Complexation of carbonate species at the goethite surface: Implications for adsorption of metal ions in natural waters. Geochimica Et Cosmochimica Acta, 58(9), 2073-2086.

van Riemsdijk, W. H., Boumans, L. J. M., \& de Haan, F. A. M. (1984). Phosphate sorption by soils: I. a model for phosphate reaction with metal-oxides in soil. Soil Science Society of America Journal, 48(3), 537-541.

Vik, E. A., Carlson, D. A., Eikum, A. S., \& Gjessing, E. T. (1984). Electrocoagulation of potable water. Water Research, 18(11), 1355-1360.

Villalobos, M., \& Leckie, J. O. (2000). Carbonate adsorption on goethite under closed and open CO2 conditions. Geochimica Et Cosmochimica Acta, 64(22), 3787-3802.

Vlyssides, A. G., Loizidou, M., Karlis, P. K., Zorpas, A. A., \& Papaioannou, D. (1999). Electrochemical oxidation of a textile dye wastewater using a $\mathrm{Pt} / \mathrm{Ti}$ electrode. Journal of Hazardous Materials, $70(1-2), 41-52$.

Vlyssides, A. G., Papaioannou, D., Loizidoy, M., Karlis, P. K., \& Zorpas, A. A. (2000). Testing an electrochemical method for treatment of textile dye wastewater. Waste Management, 20(7), 569-574.

Waltham, C. A., \& Eick, M. J. (2002). Kinetics of arsenic adsorption on goethite in the presence of sorbed silicic acid. Soil Science Society of America Journal, 66(3), $818-825$.

Wasserman, G., Liu, X., Parvez, F., Ahsan, H., Factor-Litvak, P., van Geen, A., Slavkovich, V., LoIacono, N., Cheng, Z., Hussain, I., Momotaj, H., \& J.H., G. (2004). Water arsenic exposure and children's intellectual function in Araihazar, Bangladesh. Environmental Health Perspectives, 112(13), 1329-1333.

Waychunas, G. A., Rea, B. A., Fuller, C. C., \& Davis, J. A. (1993). Surface chemistry of ferrihydrite. 1. EXAFS studies of the geometry of coprecipitated and adsorbed arsenate. Geochimica Et Cosmochimica Acta, 57(10), 2251-2269.

Webb, S. (2003). SIXPack: A graphical interface for XAS analysis using IFFEFFIT presented by Samuel Webb. Physica Scripta, T115.

Welch, A. H., Lico, M. S., \& Hughes, J. L. (1988). Arsenic in ground-water of the western United States. Ground Water, 26(3), 333-347.

WHO (1993). Guidlines for drinking-water quality. Tech. Rep. ISBN 924 154460, The World Health Organization.

WHO (2000). Towards an assessment of the socioeconomic impact of arsenic poisoning in Bangladesh. Tech. rep., World Health Organization.

WHO (2004). Guidelines for drinking water quality. Vol 1. recommendations. Tech. rep., World Health Organization. 
Wilkie, J. A., \& Hering, J. G. (1996). Adsorption of arsenic onto hydrous ferric oxide: effects of adsorbate/adsorbent ratios and co-occurring solutes. Colloids and Surfaces A: Physicochemical and Engineering Aspects, 107, 97-110.

Willett, I. R., Chartres, C. J., \& Nguyen, T. T. (1988). Migration of phosphate into aggregated particles of ferrihydrite. European Journal of Soil Science, 39(2), 275-282.

WSP-SA (2000). Arsenic mitigation in West Bengal and Bangladesh: helping households respond to a water quality crisis. Tech. rep., Water and Sanitation Program - South Asia.

Wu, S. C., \& Gschwend, P. M. (1988). Numerical modeling of sorption kinetics of organic compounds to soil and sediment particles. Water Resources Research, 24, Pages: $1373-1383$.

Xiong, Y., Strunk, P. J., Xia, H. Y., Zhu, X. H., \& Karlsson, H. T. (2001). Treatment of dye wastewater containing acid orange ii using a cell with three-phase threedimensional electrode. Water Research, 35(17), 4226-4230.

Yamauchi, H., \& Fowler, B. (1994). Toxicity and metabolism of inorganic and methylated arsenicals. In J. Nriagu (Ed.) Arsenic in the Environment, Part I. Cycling and Characterization, (p. 35). New York: Wiley-Interscience.

Yildiz, Y. S., Koparal, A. S., Irdemez, S., \& Keskinler, B. (2007). Electrocoagulation of synthetically prepared waters containing high concentration of NOM using iron cast electrodes. Journal of Hazardous Materials, 139(2), 373-380.

Zachara, J. M., Girvin, D. C., Schmidt, R. L., \& Resch, C. T. (1987). Chromate adsorption on amorphous iron oxyhydroxide in the presence of major groundwater ions. Environmental Science \& Technology, 21(6), 589-594. 


\section{Appendix A}

\section{List of Acronyms}

- ALS: Advanced Light Source (Berkeley, CA).

- EC: ElectroCoagulation.

- ECAR: ElectroChemical Arsenic Remediation.

- EGA: ECAR-Generated Adsorbent (refers to the iron (hydr)oxide sludge generated by iron dissolution and subsequent hydrolysis during the dosing stage of ECAR).

- EXAFS: Extended X-ray Absorption Fine Structure.

- FT-IR: Fourier Transform Infrared spectroscopy.

- HFO: Hydrous Ferric Oxide (a.k.a. Ferrihydrite, am-Fe(OH)$)_{3}$ ).

- PSA: Post-Synthesis Adsorption.

- PS-EGA: Post-Synthesis ECAR-Generated iron (hydr)oxide Adsorbent (i.e. EGA formed in the absence of arsenic).

- SBGW: Synthetic Bangladesh groundwater.

- SBGW-1: Synthetic Bangladesh groundwater using recipe 1, with low phosphate, no silicate, and high sulfate relative to Bangladesh averages; composition described in Table 3.1.

- SBGW-2: Synthetic Bangladesh groundwater using recipe 2, with average levels of phosphate, silicate, and sulfate relative to Bangladesh; composition described in Table 3.1.

- SEM: Scanning Electron Microscopy.

- SCE: Saturated Calomel Electrode.

- SHE: Standard Hydrogen Electrode. 
- SSRL: Stanford Synchrotron Radiation Laboratory (San Jose, CA).

- XANES: X-ray Absorption Near Edge Structure.

- XRF: X-ray Fluorescence (as in XRF maps or XRF mapping).

- XAS: X-ray Absorption Spectroscopy.

- XPS: X-ray Photon Spectroscopy.

- XRD: X-ray Diffraction.

- ZVI: Zero-Valent Iron. 